\title{
REGULARITY OF AREA MINIMIZING CURRENTS MOD $p$
}

\author{
Camillo De lellis, Jonas Hirsch, Andrea Marchese \\ AND SAlvatore Stuvard
}

\begin{abstract}
We establish a first general partial regularity theorem for area minimizing currents $\bmod (p)$, for every $p$, in any dimension and codimension. More precisely, we prove that the Hausdorff dimension of the interior singular set of an $m$-dimensional area minimizing current $\bmod (p)$ cannot be larger than $m-1$. Additionally, we show that, when $p$ is odd, the interior singular set is $(m-1)$-rectifiable with locally finite $(m-1)$-dimensional measure.
\end{abstract}

\section{Introduction}

1.1 Overview and main results. In this paper we consider currents $\bmod (p)$ (where $p \geq 2$ is a fixed positive integer), for which we follow the definitions and the terminology of [Fed69]. In particular, given an open subset $\Omega \subset \mathbb{R}^{m+n}$, we will let $\mathscr{R}_{m}(\Omega)$ and $\mathscr{F}_{m}(\Omega)$ denote the spaces of $m$-dimensional integer rectifiable currents and $m$-dimensional integral flat chains in $\Omega$, respectively. If $C \subset \mathbb{R}^{m+n}$ is a closed set (or a relatively closed set in $\Omega$ ), then $\mathscr{R}_{m}(C)$ (resp. $\mathscr{F}_{m}(C)$ ) denotes the space of currents $T \in \mathscr{R}_{m}\left(\mathbb{R}^{m+n}\right)$ (resp. $T \in \mathscr{F}_{m}\left(\mathbb{R}^{m+n}\right)$ ) with compact support $\operatorname{spt}(T)$ contained in $C$. Currents modulo $p$ in $C$ are defined introducing an appropriate family of pseudo-distances on $\mathscr{F}_{m}(C)$ : if $S, T \in \mathscr{F}_{m}(C)$ and $K \subset C$ is compact, then

$$
\begin{aligned}
\mathscr{F}_{K}^{p}(T-S):=\inf \left\{\begin{array}{l}
\mathbf{M}(R)+\mathbf{M}(Z): R \in \mathscr{R}_{m}(K), Z \in \mathscr{R}_{m+1}(K) \\
\text { such that } \left.T-S=R+\partial Z+p P \text { for some } P \in \mathscr{F}_{m}(K)\right\} .
\end{array}\right.
\end{aligned}
$$

Two flat currents in $C$ are then congruent modulo $p$ if there is a compact set $K \subset C$ such that $\mathscr{F}_{K}^{p}(T-S)=0$. The corresponding congruence class of a fixed flat chain $T$ will be denoted by $[T]$, whereas if $T$ and $S$ are congruent we will write

$$
T=S \bmod (p) .
$$

Keywords and phrases: Minimal surfaces, Area minimizing currents $\bmod (p)$, Regularity theory, Multiple valued functions, Blow-up analysis, Center manifold

Mathematics Subject Classification: 49Q15, 49Q05, 49N60, 35B65, 35J47 
The symbols $\mathscr{R}_{m}^{p}(C)$ and $\mathscr{F}_{m}^{p}(C)$ will denote the quotient groups obtained from $\mathscr{R}_{m}(C)$ and $\mathscr{F}_{m}(C)$ via the above equivalence relation. The boundary operator $\partial$ has the obvious property that, if $T=S \bmod (p)$, then $\partial T=\partial S \bmod (p)$. This allows to define an appropriate notion of boundary $\bmod (p)$ as $\partial^{p}[T]:=[\partial T]$. Correspondingly, we can define cycles and boundaries $\bmod (p)$ in $C$ :

- a current $T \in \mathscr{F}_{m}(C)$ is a cycle $\bmod (p)$ if $\partial T=0 \bmod (p)$, namely if $\partial^{p}[T]=0$;

- a current $T \in \mathscr{F}_{m}(C)$ is a boundary $\bmod (p)$ if $\exists S \in \mathscr{F}_{m+1}(C)$ such that $T=\partial S \bmod (p)$, namely $[T]=\partial^{p}[S]$.

Note that every boundary $\bmod (p)$ is a cycle $\bmod (p)$. In what follows, the closed set $C$ will always be sufficiently smooth, more precisely a complete submanifold $\Sigma$ of $\mathbb{R}^{m+n}$ without boundary and of class $C^{1}$.

Remark 1.1. Note that the congruence classes $[T]$ depend on the set $C$, and thus our notation is not precise in this regard. In particular, when two currents are congruent modulo $p$ in $\Sigma \subset \mathbb{R}^{m+n}$, then they are obviously congruent in $\mathbb{R}^{m+n}$, but the opposite implication is generally false, see also the discussion in [MS18, Remark 3.1]. However, the two properties are equivalent in the particular case of $\Sigma$ 's which are Lipschitz deformation retracts of $\mathbb{R}^{m+n}$, and we will see below that, without loss of generality, we can restrict to the latter case in most of our paper. For this reason we do not keep track of the ambient manifold in the notation regarding the $\bmod (p)$ congruence.

Definition 1.2. Let $\Omega \subset \mathbb{R}^{m+n}$ be open, and let $\Sigma \subset \mathbb{R}^{m+n}$ be a complete submanifold without boundary of dimension $m+\bar{n}$ and class $C^{1}$. We say that an $m$ dimensional integer rectifiable current $T \in \mathscr{R}_{m}(\Sigma)$ is area minimizing $\bmod (p)$ in $\Sigma \cap \Omega$ if

$$
\mathbf{M}(T) \leq \mathbf{M}(T+S) \quad \text { for every } S \in \mathscr{R}_{m}(\Omega \cap \Sigma) \text { which is a boundary } \bmod (p) .
$$

Recalling [Fed69], it is possible to introduce a suitable notion of $\operatorname{mass} \bmod (p)$ for classes $[T] \bmod (p)$, denoted by $\mathbf{M}^{p}: \mathbf{M}^{p}([T])$ is the infimum of those $t \in \mathbb{R} \cup\{+\infty\}$ such that for every $\varepsilon>0$ there are a compact set $K \subset \Sigma$ and an $S \in \mathscr{R}_{m}(\Sigma)$ with

$$
\mathscr{F}_{K}^{p}(T-S)<\varepsilon \quad \text { and } \quad \mathbf{M}(S) \leq t+\varepsilon .
$$

Analogously, [Fed69] defines the support $\bmod (p)$ of the current $T \in \mathscr{R}_{m}(\Sigma)$, by setting

$$
\operatorname{spt}^{p}(T):=\bigcap_{R=T \bmod (p)} \operatorname{spt}(R) .
$$

Clearly, the support depends only upon $[T]$, and we can thus also use the notation $\operatorname{spt}^{p}([T])$.

With the above terminology we can talk about mass minimizing classes $[T]$, because (1.1) can be rewritten as

$$
\mathbf{M}^{p}([T]) \leq \mathbf{M}^{p}\left([T]+\partial^{p}[S]\right) \quad \text { for all }[S] \text { with } \operatorname{spt}^{p}([S]) \subset \Omega \cap \Sigma .
$$


Our paper is devoted to the interior regularity theory for such objects.

Definition 1.3. Let $T$ be an area-minimizing current $\bmod (p)$ in $\Omega \cap \Sigma$. A point $q \in \Omega \cap \operatorname{spt}^{p}(T)$ is called an interior regular point if there is a neighborhood $U$ of $q$, a positive integer $Q$ and an oriented $C^{1}$ embedded submanifold $\Gamma$ of $\Sigma \cap U$ such that

(i) $T\llcorner U=Q \llbracket \Gamma \rrbracket \bmod (p)$;

(ii) $\Gamma$ has no boundary in $\Sigma \cap U$.

We will denote the set of interior regular points of $T$ by $\operatorname{Reg}(T)$.

Observe that by definition an interior regular point is necessarily contained in $\operatorname{spt}^{p}(T)$ and it is necessarily outside $\operatorname{spt}^{p}(\partial T)$. For this reason, it is natural to define the set of interior singular points of $T$ as

$$
\operatorname{Sing}(T):=\left(\Omega \cap \operatorname{spt}^{p}(T)\right) \backslash\left(\operatorname{Reg}(T) \cup \operatorname{spt}^{p}(\partial T)\right) .
$$

It is very easy to see that $\operatorname{Sing}(T)$ cannot be expected to be empty. Probably the following is the best known example: consider the three points $P_{j}:=\left(\cos \frac{2 \pi j}{3}, \sin \frac{2 \pi j}{3}\right) \in$ $\mathbb{R}^{2}$ for $j=1,2,3$ and the three oriented segments $\sigma_{j}$ in $\mathbb{R}^{2}$ joining the origin with each of them. Then $T:=\sum_{j} \llbracket \sigma_{j} \rrbracket$ is area-minimizing $\bmod (3)$ in $\mathbb{R}^{2}$ and the origin belongs to $\operatorname{Sing}(T)$.

As a first step to a better understanding of the singularities it is therefore desirable to give a bound on the Hausdorff dimension of the singular set. The present work achieves the best possible bound in the most general case, and in particular it answers a question of White, see [GMT86, Problem 4.20].

Theorem 1.4. Assume that $p \in \mathbb{N} \backslash\{0,1\}$, that $\Sigma \subset \mathbb{R}^{m+n}$ is a $C^{3, a_{0}}$ submanifold of dimension $m+\bar{n}$ for some positive $a_{0}$, that $\Omega \subset \mathbb{R}^{m+n}$ is open, and that $T \in \mathscr{R}_{m}(\Sigma)$ is area minimizing $\bmod (p)$ in $\Omega \cap \Sigma$. Then, $\mathcal{H}^{m-1+\alpha}(\operatorname{Sing}(T))=0$ for every $\alpha>0$.

Prior to the present paper, the state of the art in the literature on the size of the singular set for area minimizing currents $\bmod (p)$ was as follows. We start with the results valid in any codimension.

(a) For $m=1$ it is very elementary to see that $\operatorname{Sing}(T)$ is discrete (and empty when $p=2$ ).

(b) Under the general assumptions of Theorem 1.4, $\operatorname{Sing}(T)$ is a closed meager set in $\left(\operatorname{spt}^{p}(T) \cap \Omega\right) \backslash \operatorname{spt}^{p}(\partial T)$ by Allard's interior regularity theory for stationary varifolds, cf. [All72] (in fact, in order to apply Allard's theorem it is sufficient to assume that $\Sigma$ is of class $\left.C^{2}\right)$.

(c) For $p=2, \mathcal{H}^{m-2+\alpha}(\operatorname{Sing}(T))=0$ for every $\alpha>0$ by Federer's classical work [Fed70]; moreover the same reference shows that $\operatorname{Sing}(T)$ consists of isolated points when $m=2$; for $m>2$, Simon [Sim93, $\operatorname{Sim} 95]$ proved that $\operatorname{Sing}(T)$ is $(m-2)$-rectifiable and it has locally finite $\mathcal{H}^{m-2}$ measure.

We next look at the hypersurface case, namely $\bar{n}=1$. 
(d) When $p=2, \mathcal{H}^{m-2}(\operatorname{Sing}(T))=0$ even in the case of minimizers of general uniformly elliptic integrands, see [SSA77]; for the area functional, using [NV], one can conclude additionally that $\operatorname{Sing}(T)$ is $(m-7)$-rectifiable and has locally finite $\mathcal{H}^{m-7}$ measure.

(e) When $p=3$ and $m=2$, [Tay73] gives a complete description of $\operatorname{Sing}(T)$, which consists of $C^{1, \alpha}$ arcs where three regular sheets meet at equal angles.

(f) When $p$ is odd, [Whi86] shows that $\mathcal{H}^{m}(\operatorname{Sing}(T))=0$ for minimizers of a uniformly elliptic integrand, and that $\mathcal{H}^{m-1+\alpha}(\operatorname{Sing}(T))=0$ for every $\alpha>0$ for minimizers of the area functional.

(g) When $p=4$, [Whi79] shows that minimizers of uniformly elliptic integrands are represented by immersed manifolds outside of a closed set of zero $\mathcal{H}^{m-2}$ measure.

In view of the examples known so far it is tempting to advance the following

Conjecture 1.5. Let $T$ be as in Theorem 1.4. Denote by $\operatorname{Sing}_{f}(T)$ the subset of interior flat singular points, that is those points $q \in \operatorname{Sing}(T)$ where there is at least one flat tangent cone; see Sections 7 and 8. Then $\mathcal{H}^{m-2+\alpha}\left(\operatorname{Sing}_{f}(T)\right)=0$ for every $\alpha>0$.

Conjecture 1.5 is known to be correct for:

(a) $m=1$;

(b) $p=2$ and any $m$ and $\bar{n}$;

(c) $p$ is odd and the codimension $\bar{n}=1$.

In all three cases, however, the conjecture follows from the much stronger fact that $\operatorname{Sing}_{f}(T)$ is empty:

- the case (a) is an instructive exercise in geometric measure theory;

- the case (b) follows from Allard's regularity theorem for stationary varifold;

- the case (c) is a corollary of the main result in [Whi86].

Note that in all the other cases we cannot expect $\operatorname{Sing}_{f}(T)$ to be empty, with the easiest case being $p=4, m=2$ and $\bar{n}=1$, to be discussed in the following

EXAMPle 1.6. Consider a ball $B \subset \mathbb{R}^{2}$ as well as two distinct smooth functions $u_{1}$ and $u_{2}$ solving the minimal surfaces equation in $B$, and let $S_{1}$ and $S_{2}$ denote the integral currents in the cylinder $B \times \mathbb{R} \subset \mathbb{R}^{3}$ defined by their graphs endowed with the natural orientation. As it is well known, $S_{1}$ and $S_{2}$ are then area minimizing, both as integral currents and as currents $\bmod (2)$. Assume, in addition, that the set $\left\{u_{1}=u_{2}\right\}$ contains a curve $\gamma$ which divides $B$ into two regions $B^{>}$and $B^{<}$. Explicit $u_{1}$ and $u_{2}$ as above are easy to find. The reader could take $B$ to be a suitably small ball centered at the origin, $u_{1} \equiv 0$, and let $u_{2}$ be the function which describes Enneper's minimal surface in a neighborhood of the origin. The set $\left\{u_{1}=u_{2}\right\}$ is then given by $\{(x, y): x= \pm y\} \cap B$ and $\gamma$ can be taken to be the segment $\{x=y\} \cap B$ while $B^{>}$and $B^{<}$would then be $B \cap\{x>y\}$ and $B \cap\{x<y\}$, respectively. 
We then define the following rectifiable current $T$. Its support is the union of the graphs of $u_{1}$ and $u_{2}$, and thus of the supports of $S_{1}$ and $S_{2}$. However, while the portions of such graphs lying over $B^{>}$will be taken with the standard orientation induced by $B$, the portions lying over $B^{<}$will be taken with the opposite orientation. In $B \times \mathbb{R}$, the boundary of $T$ is $4 \llbracket \gamma \rrbracket$, and $T$ is singular along $\gamma$. Since $T$ can be written as $T=\tilde{S}_{1}+\tilde{S}_{2}$, where $\tilde{S}_{k}$ are $\bmod (2)$ equivalent to $S_{k}(k=1,2)$, and since $\tilde{S}_{k}$ are area minimizing $\bmod (2)$, the structure theorem in [Whi79] guarantees that $T$ is area minimizing $\bmod (4)$. Whenever $u_{1}$ and $u_{2}$ are chosen so that $0 \in \gamma$, $u_{1}(0)=u_{2}(0)=0$, and $\nabla u_{1}(0)=\nabla u_{2}(0)=0$ (as it is the case in the example above) then 0 is a singular point of $T$, and the (unique) tangent cone to $T$ at 0 is the two dimensional horizontal plane $\pi_{0}=\left\{x_{3}=0\right\}$ with multiplicity 2 . In such examples we thus have $0 \in \operatorname{Sing}_{f}(T)$.

In this paper we strengthen the result for $p$ odd by showing that Conjecture 1.5 in fact holds in any codimension. Indeed we prove the following more general theorem.

Theorem 1.7. Let $T$ be as in Theorem 1.4 and $Q<\frac{p}{2}$ a positive integer. Consider the subset $\operatorname{Sing}_{Q}(T)$ of $\operatorname{spt}^{p}(T) \backslash \operatorname{spt}^{p}(\partial T)$ which consists of interior singular points of $T$ where the density is $Q$ (see Definition 8.1). Then $\mathcal{H}^{m-2+\alpha}\left(\operatorname{Sing}_{Q}(T)\right)=0$ for every $\alpha>0$.

The analysis of tangent cones (cf. Corollary 7.3) implies that if $p$ is odd then

$$
\operatorname{Sing}_{f}(T) \subset \bigcup_{Q=1}^{\left\lfloor\frac{p}{2}\right\rfloor} \operatorname{Sing}_{Q}(T) .
$$

We thus get immediately

Corollary 1.8. Conjecture 1.5 holds for every $p$ odd in any dimension $m$ and codimension $\bar{n}$.

The fact above, combined with the techniques recently introduced in the remarkable work $[\mathrm{NV}]$, allows us to conclude the following theorem.

Theorem 1.9. Let $T$ be as in Theorem 1.4 and assume $p$ is odd. Then $\operatorname{Sing}(T)$ is $(m-1)$-rectifiable, and for every compact $K$ with $K \cap \operatorname{spt}^{p}(\partial T)=\emptyset$ we have $\mathcal{H}^{m-1}(\operatorname{Sing}(T) \cap K)<\infty$.

In turn the above theorem implies the following structural result.

Corollary 1.10. Let $T$ be as in Theorem 1.4 and assume in addition that $p$ is odd. Denote by $\left\{\Lambda_{i}\right\}_{i}$ the connected components of $\operatorname{spt}^{p}(T) \backslash\left(\operatorname{spt}^{p}(\partial T) \cup \operatorname{Sing}(T)\right)$. Then each $\Lambda_{i}$ is an orientable smooth minimal submanifold of $\Sigma$ and there is a choice of (smooth) orientations and multiplicities $Q_{i} \in\left[1, \frac{p}{2}\right] \cap \mathbb{N}$ such that the following properties hold for every open $U \Subset \mathbb{R}^{m+n} \backslash \operatorname{spt}^{p}(\partial T)$. 
(a) Each $T_{i}=Q_{i} \llbracket \Lambda_{i} \rrbracket$ is an integral current in $U$ and thus, having chosen an orientation $\vec{S}$ for the rectifiable set $\operatorname{Sing}(T)$, we have

$$
\left(\partial T_{i}\right)\left\llcorner U=\Theta_{i} \vec{S} \mathcal{H}^{m-1}\llcorner(\operatorname{Sing}(T) \cap U)\right.
$$

for some integer valued Borel function $\Theta_{i}$.

(b) $\sum_{i} \mathbf{M}\left(T_{i}\llcorner U)<\infty\right.$ and $T\left\llcorner U=\sum_{i} T_{i}\llcorner U\right.$.

(c) $\sum_{i} \mathbf{M}\left(\left(\partial T_{i}\right)\llcorner U)<\infty,(\partial T)\left\llcorner U=\sum_{i}\left(\partial T_{i}\right)\llcorner U\right.\right.$ and

$$
(\partial T)\left\llcorner U=\sum_{i} \Theta_{i} \vec{S} \mathcal{H}^{m-1}\llcorner(\operatorname{Sing}(T) \cap U) ;\right.
$$

in particular $\sum_{i} \Theta_{i}(q)$ is an integer multiple of $p$ for $\mathcal{H}^{m-1}$-a.e. $q \in \operatorname{Sing}(T) \cap U$.

It is tempting to advance the following conjecture.

Conjecture 1.11. The conclusions of Theorem 1.9 hold for $p$ even as well.

From the latter conjecture one can easily conclude an analogous structure theorem as in Corollary 1.10. Note that the conjecture is known to hold for $p=2$ in every codimension (in which case, in fact, we know that $\operatorname{Sing}(T)$ has dimension at most $m-2$ ) and for $p=4$ in codimension 1 .

1.2 Plan of the paper. The paper is divided into five parts: the first four parts contain the arguments leading to the proof of Theorems 1.4 and 1.7 , while the last part is concerned with the proof of the rectifiability Theorem 1.9 and of Corollary 1.10. Each part is further divided into sections. The proof of Theorems 1.4 and 1.7 is obtained by contradiction, and is inspired by F. Almgren's work on the partial regularity for area minimizing currents in any codimension as revisited by the first-named author and E. Spadaro in [DLS14, DLS16a, DLS16b]. In particular, Part 1 contains the preliminary observations and reductions aimed at stating the contradiction assumption for Theorems 1.4 and 1.7, whereas Part 2, Part 3, and Part 4 are the counterpart of the papers [DLS14, DLS16a, DLS16b], respectively. An interesting feature of the regularity theory presented in this work is that Almgren's multiple valued functions minimizing the Dirichlet energy are not the right class of functions to consider when one wants to approximate a minimizing current $\bmod (p)$ in a neighborhood of a flat interior singular point whenever the density of the point is precisely $\frac{p}{2}$. Solving this issue requires (even in the codimension $\bar{n}=1$ case) the introduction of a class of special multiple valued functions minimizing a suitably defined Dirichlet integral. The regularity theory for such maps (which we call linear theory) is the content of our paper [DLHMS]. Applications of multivalued functions to flat chains $\bmod (p)$ were already envisioned by Almgren in [Alm91], even though he considered somewhat different objects than those defined in [DLHMS]. Because of this profound interconnection between the two theories, the reading of [DLHMS] is meant to precede that of the present paper. 


\section{Almgren's regularity theory in the $\bmod (p)$ setting}

Before entering the main body of the paper, we would like to briefly present an overview of Almgren's regularity theory adapted to the setting of area minimizing currents $\bmod (p)$, focusing onto the points where major changes were required in order to overcome the intrinsic difficulties of the problem under consideration. To do so, we restrict our attention to the proof of Theorem 1.4. For the sake of simplicity, we will assume throughout this discussion that $\Sigma=\mathbb{R}^{m+\bar{n}}$.

Towards a proof by contradiction of Theorem 1.4, we exploit the classical Almgren's stratification principle for stationary varifolds in order to reduce the contradiction assumption to the following (see Proposition 8.7): there exist integers $p \geq 2$ and $Q \leq p / 2$, reals $\alpha, \eta>0$, an open ball $\Omega \ni 0$, and an $m$-dimensional rectifiable current $T$ in $\mathbb{R}^{m+\bar{n}}$ such that:

(i) $T$ is area minimizing $\bmod (p)$ in $\Omega$ with $(\partial T)\llcorner\Omega=0 \bmod (p)$ in $\Omega$ and $0 \in$ $\operatorname{Sing}_{Q}(T)$

(ii) there exist a sequence of radii $r_{k} \downarrow 0$ and an $m$-dimensional plane $\pi_{0}$ such that the integral varifolds $\mathbf{v}\left(T_{0, r_{k}}\right)$ associated with the rescaled currents centered at 0 converge to a varifold $V=Q \mathcal{H}^{m}\left\llcorner\pi_{0} \otimes \delta_{\pi_{0}}\right.$;

(iii) it holds

$$
\limsup _{k \rightarrow \infty} \mathcal{H}_{\infty}^{m-1+\alpha}\left(\operatorname{Sing}_{Q}\left(T_{0, r_{k}}\right) \cap \mathbf{B}_{1}\right) \geq \eta
$$

where $\mathbf{B}_{1}$ is the unit open ball in $\mathbb{R}^{m+\bar{n}}$.

The next step in Almgren's strategy would then be to approximate the currents $T_{k}=T_{0, r_{k}}$ with graphs of functions $u_{k}$ defined on $\pi_{0}$, taking values in the metric space $\mathcal{A}_{Q}\left(\pi_{0}^{\perp}\right)$ of $Q$-points in $\pi_{0}^{\perp}$ (that is, the space of discrete measures $T=\sum_{i=1}^{Q} \llbracket v_{i} \rrbracket$ on $\pi_{0}^{\perp}$, with positive integer coefficients and total mass $Q$ ), and minimizing a suitable linearization of the mass functional (Dir-minimizing $Q$-valued functions). In our setting, the main difficulties related to this step occur when $p$ is even and $Q=$ $p / 2$. In this case, indeed, Almgren's Dir-minimizing $Q$-valued functions are not the correct objects to perform such approximation; see [DLHMS, Example 1.2]. Notice that the phenomenon responsible of the inadequacy of classical Dir-minimizers in the approximation of area minimizing currents $\bmod (p)$ is precisely the existence of flat singular points of density $Q=p / 2$ discussed in Example 1.6. In order to introduce a class of multiple valued functions adapted to our needs, in [DLHMS] we defined the metric space $\mathscr{A}_{Q}\left(\mathbb{R}^{n}\right)$ of special $Q$-points in Euclidean space $\mathbb{R}^{n}$, and we studied the regularity properties of $\mathscr{A}_{Q}\left(\mathbb{R}^{n}\right)$-valued functions minimizing a functional representing the natural linearization of the $\operatorname{mass} \bmod (p)$ (henceforth called Dir-minimizing special $Q$-valued functions). For the reader's convenience, we briefly recall here some basic notation introduced in [DLHMS]. The space $\mathscr{A}_{Q}\left(\mathbb{R}^{n}\right)$ is defined by

$$
\mathscr{A}_{Q}\left(\mathbb{R}^{n}\right):=\mathcal{A}_{Q}\left(\mathbb{R}^{n}\right) \sqcup \mathcal{A}_{Q}\left(\mathbb{R}^{n}\right) / \sim,
$$


where $\sim$ is the equivalence relation defined by

$$
\begin{aligned}
(S, 1) \sim(T, 1) & \Longleftrightarrow S=T, \\
(S,-1) \sim(T,-1) & \Longleftrightarrow S=T, \\
(S, 1) \sim(T,-1) & \Longleftrightarrow \exists z \in \mathbb{R}^{n}: S=Q \llbracket z \rrbracket=T .
\end{aligned}
$$

Given a Borel measurable map $u: \Omega \subset \mathbb{R}^{m} \rightarrow \mathscr{A}_{Q}\left(\mathbb{R}^{n}\right)$, there is a canonical decomposition of the domain $\Omega$ into three disjoint sets $\Omega_{+}, \Omega_{-}$, and $\Omega_{0}$. More precisely, $\Omega_{0}$ is the set of points $x \in \Omega$ for which there exists $z \in \mathbb{R}^{n}$ such that $u(x)=(Q \llbracket z \rrbracket, 1)=(Q \llbracket z \rrbracket,-1) ; \Omega_{+}$and $\Omega_{-}$are, instead, the sets of points $x \in \Omega \backslash \Omega_{0}$ such that $u(x)=(S, 1)$ or $u(x)=(S,-1)$ with $S \in \mathcal{A}_{Q}\left(\mathbb{R}^{n}\right)$, respectively. Furthermore, we define the functions $u^{ \pm}: \Omega \rightarrow \mathcal{A}_{Q}\left(\mathbb{R}^{n}\right)$ by

$$
u^{ \pm}(x)= \begin{cases}S & \text { if } x \in \Omega_{ \pm} \text {and } u(x)=(S, \pm 1), \\ Q \llbracket \boldsymbol{\eta}(S) \rrbracket & \text { if } x \in \Omega \backslash \Omega_{ \pm} \text {and } u(x)=(S, \mp 1),\end{cases}
$$

where $\boldsymbol{\eta}(S)$ denotes the average of the $Q$-point $S$, see Section 3. With these notations at hand, we can define the Dirichlet energy $\operatorname{Dir}(u)$ of a $W^{1,2}$ map $u: \Omega \rightarrow \mathscr{A}_{Q}\left(\mathbb{R}^{n}\right)$ by setting

$$
\operatorname{Dir}(u):=\operatorname{Dir}\left(u^{+} \ominus \boldsymbol{\eta} \circ u\right)+\operatorname{Dir}\left(u^{-} \ominus \boldsymbol{\eta} \circ u\right)+Q \operatorname{Dir}(\boldsymbol{\eta} \circ u),
$$

where $\boldsymbol{\eta} \circ u$ is the $\left(\mathbb{R}^{n}\right.$-valued) average of $u$ and $T \ominus z:=\sum_{i=1}^{Q} \llbracket v_{i}-z \rrbracket$ if $T=$ $\sum_{i=1}^{Q} \llbracket v_{i} \rrbracket \in \mathcal{A}_{Q}\left(\mathbb{R}^{n}\right)$ and $z \in \mathbb{R}^{n}$. Moreover, we can define the integer rectifiable $m$-current $\mathbf{G}_{u}$ in $\mathbb{R}^{m+n}=\mathbb{R}^{m} \times \mathbb{R}^{n}$ associated with a Lipschitz function $u: \Omega \subset$ $\mathbb{R}^{m} \rightarrow \mathscr{A}_{Q}\left(\mathbb{R}^{n}\right)$ by:

$$
\mathbf{G}_{u}:=\mathbf{G}_{u^{+}}\left\llcorner\left(\Omega_{+} \times \mathbb{R}^{n}\right)-\mathbf{G}_{u^{-}}\left\llcorner\left(\Omega_{-} \times \mathbb{R}^{n}\right)+Q \mathbf{G}_{\boldsymbol{\eta} \circ u}\left\llcorner\left(\Omega_{0} \times \mathbb{R}^{n}\right),\right.\right.\right.
$$

where $\mathbf{G}_{u^{ \pm}}$denotes the current associated with the graph of a classical $Q$-valued function as in [DLS15, Definition 1.10] and $\mathbf{G}_{\eta \circ u}$ is the current associated with the graph of the average $\boldsymbol{\eta} \circ u$. For instance, the current $T$ described in Example 1.6 coincides with the graph $\mathbf{G}_{u}$ of the $\mathscr{A}_{2}(\mathbb{R})$-valued function defined by

$$
u(x)= \begin{cases}\left(\llbracket u_{1}(x) \rrbracket+\llbracket u_{2}(x) \rrbracket, 1\right) & \text { if } x \in B^{>} \cup \gamma, \\ \left(\llbracket u_{1}(x) \rrbracket+\llbracket u_{2}(x) \rrbracket,-1\right) & \text { if } x \in B^{<} \cup \gamma .\end{cases}
$$

Notice that, as Lipschitz $Q$-valued graphs over a domain $\Omega$ are integer rectifiable currents without boundary in the cylinder $\Omega \times \mathbb{R}^{n}$, Lipschitz special $Q$-valued graphs over $\Omega$ are integer rectifiable currents without boundary $\bmod (p)$ in $\Omega \times \mathbb{R}^{n}$.

Now that we have the correct class of approximating functions, we can get back to Almgren's program. The first step is an approximation of each current $T_{k}$ with the graph of a Lipschitz special $Q$-valued function; see Proposition 9.6. The proof is based on a BV estimate for the slices of $T_{k}$ with respect to the plane $\pi_{0}$, see Lemma 10.3: while this is classically achieved in [DLS14] testing the current with 
suitably defined differential forms, our setting requires an ad hoc proof due to the fact that $T_{k}$ may possibly have non-trivial classical boundary. The errors in such an approximation (which, we note in passing, does not use that $T_{k}$ is area minimizing $\bmod (p))$ are controlled linearly by the excess $\bmod (p)$ of $T_{k}$ with respect to the plane $\pi_{0}$ : this is defined as the difference between the mass of $T_{k}$ and the mass modulo $p$ of its projection onto $\pi_{0}$; see Definition 9.2. Exploiting the minimality of $T_{k}$, we can then substantially improve the results of this first Lipschitz approximation in two ways: first, upgrading the control of the errors in terms of a superlinear power of the excess $\bmod (p)$ (Theorem 15.1); second, showing that the approximating special $Q$-valued function is close to a Dir-minimizer (Theorem 15.4). Finally, we introduce a second notion of excess, called the nonoriented excess and smaller than the excess $\bmod (p)$ (formula (13.2)), and we show that all the error estimates in the aforementioned approximation can be upgraded replacing the excess $\bmod (p)$ with the nonoriented excess; see Theorem 16.1. The nonoriented excess is a more accurate measure of the local tilting of a current with respect to a plane regardless of orientations (much like the varifold excess), a feature that is very important in our setting, since area minimizing currents $\bmod (p)$ may exhibit changes of orientation even when their boundary $\bmod (p)$ vanishes. Furthermore, the flexibility of the nonoriented excess with respect to localization will be of vital importance in the next step of Almgren's program, namely the construction of the center manifold.

The latter is arguably the most delicate part in Almgren's proof, and it is motivated by the following issue. Given the sequence of currents $T_{k}$ converging to $Q \llbracket \pi_{0} \rrbracket$ in the sense of currents $\bmod (p)$, and given the sequence $u_{k}$ of $\mathscr{A}_{Q}\left(\pi_{0}^{\perp}\right)$-valued approximating functions, it would be tempting to perform an appropriate (nonhomogeneous) rescaling of the functions leading to a new sequence which converges, in the limit as $k \rightarrow \infty$, to a non-trivial Dir-minimizing special $Q$-valued function $u_{\infty}$. In view of (iii), if we could prove that the function $u_{\infty}$ "inherits" the singularities of the currents, then we would obtain the desired contradiction by invoking the main result of [DLHMS], namely the following

Theorem. (see [DLHMS, Theorem 10.2]) The singular set of a Dir-minimizing special $Q$-valued function defined on a domain in $\mathbb{R}^{m}$ has Hausdorff dimension at most $m-1$.

The issue in this plan is that the limit function $u_{\infty}$ may not exhibit any singularities at all: this happens when, at the natural rescaling rate of the functions $u_{k}$, the currents $T_{k}$ are centered around a smooth sheet. Conceived precisely to mod-out such a smooth sheet, the center manifold is an $m$-dimensional surface equipped with a special $Q$-valued section $N_{k}$ of its normal bundle which approximately parametrizes $T_{k}$. The blow-up argument described above then leads to the desired contradiction when performed on the approximations $N_{k}$. This portion of Almgren's proof is sufficiently robust to go through in our setting without the need of substantial modifications, with all the main necessary estimates being already available from 
the new Lipschitz approximation and the detailed analysis of Dir-minimizing special $Q$-valued functions contained in [DLHMS].

\section{Notation}

We add below a list of standard notation in Geometric Measure Theory, which will be used throughout the paper. More notation will be introduced in the main text when the need arises.

$\mathbf{B}_{r}(x)$ open ball in $\mathbb{R}^{m+n}$ centered at $x \in \mathbb{R}^{m+n}$ with radius $r>0$;

$\omega_{m}$ Lebesgue measure of the unit disc in $\mathbb{R}^{m}$;

$|A|$ Lebesgue measure of $A \subset \mathbb{R}^{m+n}$;

$\mathcal{H}^{m}$ m-dimensional Hausdorff measure in $\mathbb{R}^{m+n}$;

$\Lambda_{m}\left(\mathbb{R}^{m+n}\right)$ vector space of $m$-vectors in $\mathbb{R}^{m+n}$;

$\mathcal{D}^{m}(U)$ space of smooth differential $m$-forms with compact support in an open subset $U \subset \mathbb{R}^{m+n}$;

$\mathscr{F}_{m},\left(\mathscr{F}_{m}^{p}\right)$ integral flat chains (modulo $\left.p\right)$ of dimension $m$;

$\mathscr{R}_{m},\left(\mathscr{R}_{m}^{p}\right)$ integer rectifiable currents (modulo $p$ ) of dimension $m$; we write $T=$ $\llbracket M, \vec{\tau}, \theta \rrbracket$ if $T$ is defined by integration with respect to $\vec{\tau} \theta \mathcal{H}^{m}\llcorner M$ for a locally $\mathcal{H}^{m}$-rectifiable set $M$ oriented by the Borel measurable unit $m$-vector field $\vec{\tau}$ with multiplicity $\theta$; if $M$ is an oriented submanifold of class $C^{1}$, then we simply write $\llbracket M \rrbracket$ for the associated multiplicity one current;

$\mathscr{I}_{m},\left(\mathscr{I}_{m}^{p}\right)$ integral currents (modulo $p$ ) of dimension $m$;

$\mathbf{M},\left(\mathbf{M}^{p}\right)$ mass functional (mass modulo $\left.p\right)$;

$\|T\|,\left(\|T\|_{p}\right)$ Radon measure associated to a current $T$ (to a class $[T]$ ) with locally finite mass (mass modulo $p$ );

$\vec{T}$ Borel measurable unit $m$-vector field in the polar decomposition $T=\vec{T}\|T\|$ of a current with locally finite mass; if $T=\llbracket M, \vec{\tau}, \theta \rrbracket$ is rectifiable, then $\vec{T}=\operatorname{sgn}(\theta) \vec{\tau}\|T\|$-a.e., so that $\vec{T}$ is an orientation of $M$;

$T\llcorner A$ restriction of the current $T$ to the set $A$ : well defined for any Borel $A$ when $T$ has locally finite mass, and for $A$ open if $T$ is any current;

$\langle T, f, z\rangle$ slice of the current $T$ with the function $f$ at the point $z$;

$f_{\sharp} T$ push-forward of the current $T$ through the map $f$;

$\Theta^{m}(\mu, x) m$-dimensional density of the measure $\mu$ at the point $x$, given by $\Theta^{m}(\mu, x)$ $:=\lim _{r \rightarrow 0^{+}} \frac{\mu\left(\mathbf{B}_{r}(x)\right)}{\omega_{m} r^{m}}$ when the limit exists;

$\Theta_{T}(x), \Theta(T, x)$ same as $\Theta^{m}(\|T\|, x)$ if $T$ is an $m$-dimensional current with locally finite mass;

$\operatorname{spt}(\mu)$ support of $\mu$, where $\mu$ is a Radon measure on $\mathbb{R}^{m+n}$ : it is defined as the set of all points $x \in \mathbb{R}^{m+n}$ such that $\mu\left(\mathbf{B}_{r}(x)\right)>0$ for all $r>0$; $\operatorname{spt}(T)$ same as $\operatorname{spt}(\|T\|)$ if $T$ is a current with locally finite mass; 
$\operatorname{spt}^{p}(T)$ support $\bmod (p)$ of an integer rectifiable current $T$ : it only depends on the equivalence class $[T]$;

$\mathbf{v}(M, \Theta)$ rectifiable $m$-varifold defined by $\Theta \mathcal{H}^{m}\left\llcorner M \otimes \delta_{T . M}\right.$ for a locally $\mathcal{H}^{m}$ rectifiable set $M$ and a locally $\mathcal{H}^{m}\llcorner M$-integrable multiplicity $\Theta$;

$\mathbf{v}(T)$ integral varifold associated to an integer rectifiable current $T$ : if $T=\llbracket M, \vec{\tau}, \theta \rrbracket$, then $\mathbf{v}(T)=\mathbf{v}(M,|\theta|) ;$

$\delta V[X]$ first variation of the varifold $V$ in the direction of the vector field $X$;

$A_{\Sigma}$ second fundamental form of a submanifold $\Sigma \subset \mathbb{R}^{m+n}$;

$H_{\Sigma}$ mean curvature of a submanifold $\Sigma \subset \mathbb{R}^{m+n}$;

$\operatorname{Lip}(X, Y)$ space of Lipschitz functions $f: X \rightarrow Y$, where $X, Y$ are metric spaces;

$\operatorname{Lip}(f)$ Lipschitz constant of the Lipschitz function $f$;

$\left(\mathcal{A}_{Q}\left(\mathbb{R}^{n}\right), \mathcal{G}\right)$ metric space of classical $Q$-points in $\mathbb{R}^{n}$

$\left(\mathscr{A}_{Q}\left(\mathbb{R}^{n}\right), \mathcal{G}_{s}\right)$ metric space of special $Q$-points in $\mathbb{R}^{n}$;

$\boldsymbol{\eta}(S)$ average of the $Q$-point $S$, so that if $S=\sum_{i=1}^{Q} \llbracket S_{i} \rrbracket \in \mathcal{A}_{Q}\left(\mathbb{R}^{n}\right)$ then $\boldsymbol{\eta}(S)=Q^{-1} \sum_{i=1}^{Q} S_{i} \in \mathbb{R}^{n}$

$\boldsymbol{\eta} \circ f$ average of the (possibly special) multiple valued function $f$;

$\operatorname{Gr}(u)$ set-theoretical graph of a (possibly multi-valued) function $u$;

$\mathbf{T}_{F}$ integer rectifiable current associated (via push-forward) to the image of a (possibly special) multiple valued function;

$\mathbf{G}_{u}$ integer rectifiable current associated to the graph of a (possibly special) multiple valued function.

\section{Part 1. Preliminary observations and blow-up sequence}

\section{Preliminary reductions}

We recall first that, as specified in [Fed69, 4.2.26], for any $S \in \mathscr{R}_{m}(\Sigma)$ we can find a representative $\bmod (p)$, namely a $T \in \mathscr{R}_{m}(\Sigma)$ congruent to $S \bmod (p)$ such that

$$
\|T\|(A) \leq \frac{p}{2} \mathcal{H}^{m}(A) \quad \text { for every Borel } A \subset \Sigma .
$$

In particular, such a representative has multiplicity function $\theta$ such that $|\theta| \leq p / 2$ at $\|T\|$-a.e. point, and it satisfies $\mathbf{M}^{p}([T\llcorner U])=\|T\|(U)$ for every open set $U$ and $\operatorname{spt}(T)=\operatorname{spt}^{p}(T)$ (observe in passing that the restriction to an open set $U$ is defined for every current). It is evident that if $T \in \mathscr{R}_{m}(\Sigma)$ is area minimizing $\bmod (p)$ in $\Omega \cap \Sigma$ then $T$ is necessarily representative $\bmod (p)$ in $\Omega \cap \Sigma$, in the sense that (4.1) holds true for every Borel $A \subset \Omega \cap \Sigma$. For this reason, we shall always assume that $T$ is representative $\bmod (p)$, and that the aforementioned properties concerning multiplicity, mass and support of $T$ are satisfied. Note also that such $T$ is area minimizing $\bmod (p)$ in any smaller open set $U \subset \Omega$. Moreover $T$ is area $\operatorname{minimizing} \bmod (p)$ in $\Omega$ if and only if $T\llcorner\Omega$ is area $\operatorname{minimizing} \bmod (p)$ in $\Omega$. Also, for $\Omega$ sufficiently small the 
regularity of $\Sigma$ guarantees that $\Sigma \cap \Omega$ is a graph, and thus, if in addition $\Omega$ is a ball, $\overline{\Sigma \cap \Omega}$ is a Lipschitz deformation retract of $\mathbb{R}^{m+n}$. A current $S \in \mathscr{R}_{m}(\Sigma \cap \Omega)$ is thus a cycle $\bmod (p)$ if and only if it is a cycle $\bmod (p)$ in $\mathbb{R}^{m+n}$. In these circumstances it does not matter what the shape of the ambient manifold $\Sigma$ is outside $\Omega$ and thus, without loss of generality, we can assume that $\Sigma$ is in fact an entire graph. By the same type of arguments we can also assume that $\partial^{p}[T]=0$ in $\Omega$. We summarize these reductions in the following assumption (which will be taken as a hypothesis in most of our statements) and in a lemma (which will be used repeatedly).

Assumption 4.1. $\Sigma$ is an entire $C^{3, a_{0}}(m+\bar{n})$-dimensional graph in $\mathbb{R}^{m+n}$ with $0<a_{0} \leq 1$, and $\Omega \subset \mathbb{R}^{m+n}$ is an open ball. $T$ is an $m$-dimensional representative $\bmod (p)$ in $\Sigma$ that is area minimizing $\bmod (p)$ in $\Sigma \cap \Omega$ and such that $(\partial T)\llcorner\Omega=0$ $\bmod (p)$ in $\Omega$.

Lemma 4.2. Let $\Omega, \Sigma$ and $T$ be as in Assumption 4.1. Let $T^{\prime} \in \mathscr{R}_{m}(\Sigma)$ be such that $\operatorname{spt}\left(T^{\prime}-T\right) \subset \Omega$ and $\partial T^{\prime}=\partial T \bmod (p)$. Then

$$
\mathbf{M}\left(T\llcorner\Omega) \leq \mathbf{M}\left(T^{\prime}\llcorner\Omega) .\right.\right.
$$

Theorem 1.4 is then equivalent to

Theorem 4.3. Under the Assumption 4.1 Sing $(T)$ has Hausdorff dimension at most $m-1$.

\section{$5 \quad$ Stationarity and compactness}

Another important tool that will be used repeatedly in the sequel is the fact that the integral varifold $\mathbf{v}(T)$ induced by an area minimizing representative $\bmod (p) T$ is stationary in the open set $\Omega \cap \Sigma \backslash \operatorname{spt}^{p}(\partial T)$.

Lemma 5.1. Let $\Omega, \Sigma$ and $T$ be as in Assumption 4.1. Then $\mathbf{v}(T)$ is stationary in $\Sigma \cap \Omega$, namely

$$
\delta \mathbf{v}(T)[X]=0 \quad \text { for all } X \in C_{c}^{1}\left(\Omega, \mathbb{R}^{m+n}\right) \text { tangent to } \Sigma .
$$

More generally, for $X \in C_{c}^{1}\left(\Omega, \mathbb{R}^{m+n}\right)$ we have

$$
\delta \mathbf{v}(T)[X]=-\int X \cdot \vec{H}_{T}(x) d\|T\|(x),
$$

where the mean curvature vector $\vec{H}_{T}$ can be explicitly computed from the second fundamental form $A_{\Sigma}$ of $\Sigma$. More precisely, if the orienting vector field of $T$ is $\vec{T}(x)=v_{1} \wedge \ldots \wedge v_{m}$ and $v_{i}$ are orthonormal, then

$$
\vec{H}_{T}(x)=\sum_{i=1}^{m} A_{\Sigma}\left(v_{i}, v_{i}\right) .
$$


Proof. Consider a diffeomorphism $\Phi$ of $\Omega$ such that $\Phi(\Sigma \cap \Omega) \subset \Sigma \cap \Omega$ and $\left.\Phi\right|_{\Omega \backslash K} \equiv$ $\left.\operatorname{id}\right|_{\Omega \backslash K}$ for some compact set $K \subset \Sigma \cap \Omega$. The current $\Phi_{\sharp} T$ satisfies $\operatorname{spt}\left(T-\Phi_{\sharp} T\right) \Subset \Sigma \cap$ $\Omega$. Moreover, since $\partial\left(\Phi_{\sharp} T\right)=\Phi_{\sharp}(\partial T)$ and $\partial T=0 \bmod (p)$, also $\partial\left(\Phi_{\sharp} T\right)=0 \bmod (p)$, so that, in particular,

$$
\partial\left(\Phi_{\sharp} T\right)=\partial T \bmod (p) .
$$

From (4.2), and setting $V:=\mathbf{v}(T)$, we then get

$$
\|V\|(\Omega)=\mathbf{M}\left(T\llcorner\Omega) \leq \mathbf{M}\left(\Phi_{\sharp} T\llcorner\Omega)=\left\|\Phi_{\sharp} V\right\|(\Omega) .\right.\right.
$$

This easily implies that $V$ is stationary in $\Sigma \cap \Omega$.

The second claim of the Lemma follows then from the stationarity of $V$ in $\Sigma$, see for instance [Sim83].

Consider now an open ball $\mathbf{B}_{R}=\Omega \subset \mathbb{R}^{m+n}$, a sequence of Riemannian manifolds $\Sigma_{k}$ and a sequence of currents $T_{k}$ such that each triple $\left(\Omega, \Sigma_{k}, T_{k}\right)$ satisfies the Assumption 4.1. In addition assume that:

(a) $\Sigma_{k}$ converges locally strongly in $C^{2}$ to a Riemannian submanifold $\Sigma$ of $\mathbb{R}^{m+n}$ which is also an entire graph;

(b) $\sup _{k}\left\|T_{k}\right\|\left(\mathbf{B}_{R}\right)=\sup _{k} \mathbf{M}^{p}\left(T_{k}\left\llcorner\mathbf{B}_{R}\right)<\infty\right.$;

(c) $\sup _{k} \mathbf{M}^{p}\left(\partial\left(T_{k}\left\llcorner\mathbf{B}_{R}\right)\right)<\infty\right.$.

By the compactness theorem for integral currents $\bmod (p)$ (cf. [Fed69, Theorem $(4.2 .17)^{\nu}$, p. 432]), we conclude the existence of a subsequence, not relabeled, of a current $T \in \mathscr{R}_{m}\left(\mathbb{R}^{m+n}\right)$ and of a compact set $K \supset \mathbf{B}_{R}$ such that

$$
\lim _{k \rightarrow \infty} \mathscr{F}_{K}^{p}\left(T_{k}\left\llcorner\mathbf{B}_{R}-T\right)=0\right.
$$

and

$$
(\partial T)\left\llcorner\mathbf{B}_{R}=0 \quad \bmod (p) .\right.
$$

Let $U_{\delta}$ be the closure of the $\delta$-neighborhood of $\Sigma$ and consider that, for a sufficiently small $\delta>0$, the compact set $K^{\prime}:=\overline{\mathbf{B}}_{R} \cap U_{\delta}$ is a Lipschitz deformation retract of $\mathbb{R}^{m+n}$. For $k$ sufficiently large, the currents $T_{k}\left\llcorner\mathbf{B}_{R}\right.$ are supported in $K^{\prime}$ and [Fed69, Theorem $\left.(4.2 .17)^{\nu}\right]$ implies that $\operatorname{spt}(T) \subset K^{\prime}$. Since $\delta$ can be chosen arbitrarily small, we conclude that $\operatorname{spt}(T) \subset \Sigma$ and hence that $T \in \mathscr{R}_{m}(\Sigma)$.

At the same time, by Allard's compactness theorem for stationary integral varifolds, we can assume, up to extraction of a subsequence, that $\mathbf{v}\left(T_{k}\left\llcorner\mathbf{B}_{R}\right)\right.$ converges to some integral varifold $V$ in the sense of varifolds.

Proposition 5.2. Consider $\Omega, \Sigma_{k}, T_{k}, \Sigma, T$ and $V$ as above. Then

(i) $T$ is minimizing $\bmod (p)$ in $\Omega \cap \Sigma$, so that, in particular, $T$ is representative $\bmod (p)$;

(ii) $V=\mathbf{v}(T)$ is the varifold induced by $T$. 
Proof. Let us simplify the notation by writing $T_{k}$ in place of $T_{k}\left\llcorner\mathbf{B}_{R}\right.$. Recall that $\mathscr{F}_{K}^{p}\left(T_{k}-T\right) \rightarrow 0$ for some compact set $K \supset \mathbf{B}_{R}$. This means that there are sequences of rectifiable currents $R_{k}, S_{k}$ and integral currents $Q_{k}{ }^{1}$ with support in $K$ such that

$$
T_{k}-T=R_{k}+\partial S_{k}+p Q_{k}
$$

and

$$
\lim _{k \rightarrow \infty}\left(\mathbf{M}\left(R_{k}\right)+\mathbf{M}\left(S_{k}\right)\right)=0 .
$$

As above, denote by $U_{\delta}$ the closure of the $\delta$-neighborhood of the submanifold $\Sigma$. Observe next that, for every $\delta$ sufficiently small, $K_{\delta}:=U_{\delta} \cap \overline{\mathbf{B}}_{R}$ is a Lipschitz deformation retract. Moreover, for each $k$ sufficiently large $\operatorname{spt}\left(T_{k}\right) \subset K_{\delta}$. We can thus assume, without loss of generality, the existence of a $\bar{k}(\delta) \in \mathbb{N}$ such that

$$
\operatorname{spt}\left(R_{k}\right), \operatorname{spt}\left(S_{k}\right), \operatorname{spt}\left(Q_{k}\right) \subset K_{\delta} \quad \forall k \geq \bar{k}(\delta) .
$$

Next, if we denote by $U_{\delta, k}$ the closures of the $\delta$-neighborhoods of $\Sigma_{k}$, due to their $C^{2}$ regularity and $C^{2}$ convergence to $\Sigma$, for a $\delta>0$ sufficiently small (independent of $k$ ) the nearest point projections

$$
\mathbf{p}_{k}: U_{\delta, k} \rightarrow \Sigma_{k}
$$

are well defined. Moreover,

$$
\lim _{\sigma \downarrow 0} \sup _{k} \operatorname{Lip}\left(\left.\mathbf{p}_{k}\right|_{U_{\sigma, k}}\right)=1
$$

We now show that $T$ is area minimizing $\bmod (p)$ in $\mathbf{B}_{R} \cap \Sigma$. Assume not: then there is a $\rho<R$ and a current $\hat{T}$ with $\operatorname{spt}(T-\hat{T}) \subset \overline{\mathbf{B}}_{\rho} \cap \Sigma$ such that

$$
\partial \hat{T}=\partial T \bmod (p)
$$

and, for every $s \in] \rho, R[$,

$$
\varepsilon:=\mathbf{M}\left(T\left\llcorner\mathbf{B}_{s}\right)-\mathbf{M}\left(\hat{T}\left\llcorner\mathbf{B}_{s}\right)>0,\right.\right.
$$

where $\varepsilon$ is independent of $s$ because of the condition $\operatorname{spt}(T-\hat{T}) \subset \overline{\mathbf{B}}_{\rho}$.

Denote by $d: \mathbb{R}^{m+n} \rightarrow \mathbb{R}$ the map $x \mapsto|x|$ and consider the slices $\left\langle S_{k}, d, s\right\rangle$. By Chebyshev's inequality, for each $k$ we can select an $\left.s_{k} \in\right] \rho, \frac{R+\rho}{2}[$ such that

$$
\mathbf{M}\left(\left\langle S_{k}, d, s_{k}\right\rangle\right) \leq \frac{2}{R-\rho} \mathbf{M}\left(S_{k}\right)
$$

Consider therefore the current:

$$
\hat{T}_{k}:=T_{k}\left\llcorner\left(\mathbb{R}^{m+n} \backslash \mathbf{B}_{s_{k}}\right)-\left\langle S_{k}, d, s_{k}\right\rangle+R_{k}\left\llcorner\mathbf{B}_{s_{k}}+\hat{T}\left\llcorner\mathbf{B}_{s_{k}} .\right.\right.\right.
$$

1 Although the definition of flat convergence modulo $p$ is given with $Q_{k}$ flat chains, a simple density argument shows that we can in fact take them integral. 
Observe first that $\operatorname{spt}\left(T_{k}-\hat{T}_{k}\right) \subset \mathbf{B}_{\frac{R+\rho}{2}}$. Also, note that (5.5) implies that $\partial S_{k}$ has finite mass. Hence, by [Sim83, Lemma 28.5(2)],

$$
\left\langle S_{k}, d, s_{k}\right\rangle=\partial\left(S_{k}\left\llcorner\mathbf{B}_{s_{k}}\right)-\left(\partial S_{k}\right)\left\llcorner\mathbf{B}_{s_{k}} .\right.\right.
$$

In particular, combining the latter equality with (5.5), we get

$$
\begin{aligned}
\partial \hat{T}_{k}: & =\partial\left(T_{k}\left\llcorner\mathbb{R}^{m+n} \backslash \mathbf{B}_{s_{k}}\right)+\partial\left(\left(T_{k}-T-R_{k}-p Q_{k}\right)\left\llcorner\mathbf{B}_{s_{k}}\right)+\partial\left(R_{k}\left\llcorner\mathbf{B}_{s_{k}}\right)+\partial\left(\hat{T}\left\llcorner\mathbf{B}_{s_{k}}\right)\right.\right.\right.\right. \\
& =\partial T_{k}-p \partial\left(Q_{k}\left\llcorner\mathbf{B}_{s_{k}}\right)+\partial(\hat{T}-T),\right.
\end{aligned}
$$

where in the second line we have used that $\operatorname{spt}(\hat{T}-T) \subset \mathbf{B}_{\rho} \subset \mathbf{B}_{s_{k}}$. Since $\partial(\hat{T}-T)=$ $0 \bmod (p)$ in $\Sigma \subset \mathbb{R}^{m+n}$, we conclude that $\partial\left(\hat{T}_{k}-T_{k}\right)=0 \bmod (p)$ in $\mathbb{R}^{m+n}$. However, considering (5.7), for $k$ large enough the currents $\hat{T}_{k}, S_{k}, R_{k}, Q_{k}, T$ and $\hat{T}$ are all supported in the domain of definition of the retraction $\mathbf{p}_{k}$. Since $\left(\mathbf{p}_{k}\right)_{\sharp} T_{k}=T_{k}$, we then have that $\partial\left(T_{k}-\left(\mathbf{p}_{k}\right)_{\sharp} \hat{T}_{k}\right)=0 \bmod (p)$ in $\Sigma_{k}$. Consider also that, for each $\sigma>0$ fixed, there is a $\bar{k}(\sigma) \in \mathbb{N}$ such that all the currents above are indeed supported in $U_{\sigma, k}$ when $k \geq \bar{k}(\sigma)$. This implies in particular that, by (5.8),

$$
\liminf _{k \uparrow \infty} \mathbf{M}\left(\left(\mathbf{p}_{k}\right)_{\sharp} \hat{T}_{k}\right)=\liminf _{k \uparrow \infty} \mathbf{M}\left(\hat{T}_{k}\right) .
$$

Up to extraction of a subsequence, we can assume that $s_{k} \rightarrow s$ for some $s \in\left[\rho, \frac{R+\rho}{2}\right]$. Recalling the semicontinuity of the $p$-mass with respect to the flat convergence $\bmod (p)$, we easily see that $\left(\right.$ since the $T_{k}$ 's and $T$ are all representative $\bmod (p)$ )

$$
\liminf _{k \rightarrow \infty} \mathbf{M}\left(T_{k}\left\llcorner\mathbf{B}_{s_{k}}\right) \geq \mathbf{M}\left(T\left\llcorner\mathbf{B}_{s}\right) .\right.\right.
$$

Next, by the estimates (5.10) and (5.6) we immediately gain

$$
\liminf _{k \uparrow \infty}\left(\mathbf{M}\left(\hat{T}_{k}\right)-\mathbf{M}\left(T_{k}\right)\right) \leq-\varepsilon
$$

Finally, since the map $\mathbf{p}_{k}$ is the identity on $\Sigma_{k}$, again thanks to (5.8) and to the observation on the supports of $\hat{T}_{k}-T_{k}$, it turns out that $\operatorname{spt}\left(\left(\mathbf{p}_{k}\right)_{\sharp} \hat{T}_{k}-T_{k}\right) \subset \Sigma_{k} \cap \mathbf{B}_{R}$ for $k$ large enough. We thus have contradicted the minimality of $T_{k}$.

Observe that, if in the argument above we replace $\hat{T}$ with $T$ itself, we easily achieve that, for every fixed $\rho>0$, there is a sequence $\left.\left\{s_{k}\right\} \subset\right] \rho, \frac{R+\rho}{2}$ [ converging to some $s \in\left[\rho, \frac{R+\rho}{2}\right]$, with the property that

$$
\liminf _{k \uparrow \infty}\left(\mathbf { M } \left(T\left\llcorner\mathbf{B}_{s_{k}}\right)-\mathbf{M}\left(T_{k}\left\llcorner\mathbf{B}_{s_{k}}\right)\right) \geq 0 .\right.\right.
$$

By this and by the semicontinuity of the $p$-mass under flat convergence, we easily conclude that

$$
\lim _{k \rightarrow \infty}\left\|T_{k}\right\|\left(\mathbf{B}_{\rho}\right)=\|T\|\left(\mathbf{B}_{\rho}\right) \quad \text { for every } \rho<R \text {. }
$$


The latter implies then that $\left\|T_{k}\right\| \stackrel{*}{\rightarrow}\|T\|$ in the sense of measures in $\mathbf{B}_{R}$. Consider now the rectifiable sets $E_{k}, E$ and the Borel functions $\Theta_{k}: E_{k} \rightarrow \mathbb{N} \backslash\{0\}, \Theta: E \rightarrow$ $\mathbb{N} \backslash\{0\}$ such that

$$
\left\|T_{k}\right\|=\Theta_{k} \mathcal{H}^{m}\left\llcorner E_{k}, \quad\|T\|=\Theta \mathcal{H}^{m}\llcorner E .\right.
$$

Let $T_{q} E_{k}$ (resp. $T_{q} E$ ) be the approximate tangent space to $E_{k}$ (resp. $E$ ) at $\mathcal{H}^{m}$-a.e. point $q$. The varifold $\mathbf{v}\left(T_{k}\right)$ is then defined to be $\Theta_{k} \mathcal{H}^{m}\left\llcorner E_{k} \otimes \delta_{T_{q} E_{k}}\right.$. If the varifold limit $V$ is given by $\Theta^{\prime} \mathcal{H}^{m}\left\llcorner F \otimes \delta_{T_{q} F}\right.$, we then know that $\left\|V_{k}\right\| \stackrel{*}{\rightarrow}\|V\|=\Theta^{\prime} \mathcal{H}^{m}\llcorner F$. But since $\left\|V_{k}\right\|=\left\|T_{k}\right\|$, we then know that $\mathcal{H}^{m}((F \backslash E) \cup(E \backslash F))=0$ and that $\Theta^{\prime}=\Theta \mathcal{H}^{m}$-almost everywhere. This shows then that $V=\mathbf{v}(T)$.

\section{Slicing formula $\bmod (p)$}

In this section we prove a suitable version of the slicing formula for currents $\bmod (p)$, which will be useful in several contexts. We let $\mathscr{I}_{m}^{p}(C)$ denote the group of integral currents $\bmod (p)$, that is of classes $[T] \in \mathscr{R}_{m}^{p}(C)$ such that $\partial^{p}[T] \in \mathscr{R}_{m-1}^{p}(C)$.

Lemma 6.1. Let $\Omega \subset \mathbb{R}^{m+n}$ be a bounded ball, let $[T] \in \mathscr{I}_{m}^{p}(\bar{\Omega})$ be an integral current $\bmod (p)$, and let $f: \bar{\Omega} \rightarrow \mathbb{R}$ be a Lipschitz function. If $T \in \mathscr{R}_{m}(\bar{\Omega})$ is any rectifiable representative of $[T]$ and $Z \in \mathscr{R}_{m-1}(\bar{\Omega})$ is any rectifiable representative of $[\partial T]$, then the following holds for a.e. $t \in \mathbb{R}$ :

(i) $\langle T, f, t\rangle=\partial(T\llcorner\{f<t\})-Z\llcorner\{f<t\} \bmod (p)$;

(ii) $\langle T, f, t\rangle$ is a representative $\bmod (p)$ if $T$ is a representative $\bmod (p)$;

(iii) if $T$ is a representative $\bmod (p)$, and if $\partial T=0 \bmod (p)$, then

$$
\mathbf{M}(\langle T, f, t\rangle)=\mathbf{M}^{p}(\partial(T\llcorner\{f<t\})) .
$$

Before coming to the proof of Lemma 6.1 we wish to point out two elementary consequences of the theory of currents $\bmod (p)$ which are going to be rather useful in the sequel.

LEMma 6.2. If $T$ is an integer rectifiable $m$-dimensional current in $\mathbb{R}^{m+n}$ and $f$ : $\mathbb{R}^{m+n} \rightarrow \mathbb{R}^{k}$ is a Lipschitz map with $k \leq m$, then:

(i) $T$ is a representative $\bmod (p)$ if and only if the density of $T$ is at most $\frac{p}{2}\|T\|$-a.e.

(ii) If $T$ is a representative $\bmod (p)$, then $\langle T, f, t\rangle$ is a representative $\bmod (p)$ for a.e. $t \in \mathbb{R}^{k}$.

(iii) If $n=0$ and $\operatorname{spt}(T) \subset K$ for a compact set $K$, then $\mathscr{F}_{K}^{p}(T)=\mathbf{M}^{p}(T)$.

(iv) Let $T$ be as in (iii) and in particular $T=\Theta \llbracket K \rrbracket$, where $\Theta$ is integer valued. If we let

$$
|\Theta(x)|_{p}:=\min \{|\Theta(x)-k p|: k \in \mathbb{Z}\},
$$

then

$$
\mathbf{M}^{p}\left(T\llcorner E)=\int_{E}|\Theta(x)|_{p} d x \quad \text { for all Borel } E \subset \mathbb{R}^{m}\right.
$$


Proof. (i) is an obvious consequence of Federer's characterization in [Fed69]: an integer rectifiable current $T$ of dimension $m$ is a representative $\bmod (p)$ if and only if $\|T\|(E) \leq \frac{p}{2} \mathcal{H}^{m}(E)$ for every Borel set $E$. By the coarea formula for rectifiable sets, this property is preserved for a.e. slice and thus (ii) is immediate. Moreover, again by Federer's characterization, if $T$ is as in (iv), and if $k(x)=\arg \min \{\mid \Theta(x)-$ $k p \mid: k \in \mathbb{Z}\}$, then setting $\Theta^{\prime}(x):=\Theta(x)-k(x) p$ we have that $T^{\prime}=\Theta^{\prime} \llbracket K \rrbracket$ is a representative $\bmod (p)$ of $T$, and thus, since $\left|\Theta^{\prime}\right|=|\Theta|_{p}$, (6.2) follows directly from $\mathbf{M}^{p}\left(T\llcorner E)=\left\|T^{\prime}\right\|(E)\right.$.

As for (iii), since $T$ is a top-dimensional current, $\mathscr{R}_{m+1}(K)=\{0\}$. We thus have $\mathscr{F}_{K}^{p}(T)=\inf \left\{\mathbf{M}(R): T=R+p P \quad\right.$ for some $R \in \mathscr{R}_{m}(K)$ and $\left.P \in \mathscr{F}_{m}(K)\right\}$.

Observe however that, since $K$ is $m$-dimensional, $\mathscr{F}_{m}(K)$ consists of the integer rectifiable currents with support in $K$. A simple computation gives then

$$
\mathscr{F}_{K}^{p}(T)=\int_{K}|\Theta(x)|_{p} d x
$$

and we can use (iv) to conclude.

Proof of Lemma 6.1. (ii) has been addressed already in Lemma 6.2, and (iii) is a simple consequence of Lemma 6.2 and of (i) with the choice $Z=0$.

We now come to the proof of (i). By [MS18, Theorem 3.4], there exists a sequence $\left\{P_{k}\right\}_{k=1}^{\infty}$ of integral polyhedral chains and currents $R_{k} \in \mathscr{R}_{m}(\bar{\Omega}), S_{k} \in \mathscr{R}_{m+1}(\bar{\Omega})$ and $Q_{k} \in \mathscr{I}_{m}(\bar{\Omega})$, with the following properties for every $k \geq 1$ :

$$
\begin{gathered}
T-P_{k}=R_{k}+\partial S_{k}+p Q_{k}, \\
\mathbf{M}^{p}\left(P_{k}\right) \leq \mathbf{M}^{p}(T)+\frac{1}{k 2^{k}}, \\
\mathbf{M}^{p}\left(\partial P_{k}\llcorner\Omega) \leq \mathbf{M}^{p}\left(\partial T\llcorner\Omega)+\frac{1}{k 2^{k}},\right.\right. \\
\mathbf{M}\left(R_{k}\right)+\mathbf{M}\left(S_{k}\right) \leq \frac{2}{k 2^{k}} .
\end{gathered}
$$

Since $P_{k}$ is an integral current, by the classical slicing theory (cf. for instance [Sim83, Lemma 28.5(2)]), the following formula holds for a.e. $t \in \mathbb{R}$ :

$$
\left\langle P_{k}, f, t\right\rangle=\partial\left(P_{k}\llcorner\{f<t\})-\left(\partial P_{k}\right)\llcorner\{f<t\} .\right.
$$

The identity (6.3) implies that $\partial S_{k}$ has locally finite mass, and thus $S_{k}$ is an integral current. In particular, $\partial\left\langle S_{k}, f, t\right\rangle=-\left\langle\partial S_{k}, f, t\right\rangle$. Furthermore, the slicing formula holds true for $S_{k}$ as well, that is for a.e. $t \in \mathbb{R}$ one has:

$$
\left\langle S_{k}, f, t\right\rangle=\partial\left(S_{k}\llcorner\{f<t\})-\left(\partial S_{k}\right)\llcorner\{f<t\} .\right.
$$


Since $Z=\partial T \bmod (p)$, there exist currents $\tilde{R}_{k} \in \mathscr{R}_{m-1}(\bar{\Omega}), \tilde{S}_{k} \in \mathscr{R}_{m}(\bar{\Omega})$ and $\tilde{Q}_{k} \in \mathscr{I}_{m-1}(\bar{\Omega})$ such that for every $k \geq 1$ :

$$
\begin{aligned}
& Z-\partial T=\tilde{R}_{k}+\partial \tilde{S}_{k}+p \tilde{Q}_{k}, \\
& \mathbf{M}\left(\tilde{R}_{k}\right)+\mathbf{M}\left(\tilde{S}_{k}\right) \leq \frac{1}{k 2^{k}} .
\end{aligned}
$$

Combining (6.3) and (6.9), we can therefore write:

$$
\begin{aligned}
Z-\partial P_{k} & =\partial T-\partial P_{k}+Z-\partial T \\
& =\tilde{R}_{k}+\partial\left(R_{k}+\tilde{S}_{k}\right)+p\left(\partial Q_{k}+\tilde{Q}_{k}\right) .
\end{aligned}
$$

The identity (6.11) implies that $\partial\left(R_{k}+\tilde{S}_{k}\right)$ has locally finite mass, and thus in particular $R_{k}+\tilde{S}_{k}$ is an integral current. Hence, for a.e. $t \in \mathbb{R}$ the slicing formula holds true for $R_{k}+\tilde{S}_{k}$, that is:

$$
\left\langle R_{k}+\tilde{S}_{k}, f, t\right\rangle=\partial\left(\left(R_{k}+\tilde{S}_{k}\right)\llcorner\{f<t\})-\left(\partial\left(R_{k}+\tilde{S}_{k}\right)\right)\llcorner\{f<t\} .\right.
$$

From the identities (6.3) and (6.11), and using (6.7), (6.8), (6.12), and the slicing formula for $Q_{k}$ we easily conclude that the following holds for a.e. $t \in \mathbb{R}$ :

$$
\begin{aligned}
& \langle T, f, t\rangle-\partial(T\llcorner\{f<t\})+Z\llcorner\{f<t\} \\
= & \tilde{R}_{k}\left\llcorner\{f<t\}-\left\langle\tilde{S}_{k}, f, t\right\rangle+\partial\left(\tilde{S}_{k}\llcorner\{f<t\})+p \tilde{Q}_{k}\llcorner\{f<t\} .\right.\right.
\end{aligned}
$$

Now, $\tilde{Q}_{k}\llcorner\{f<t\}$ is an integral current and thus, setting $K:=\bar{\Omega}$, we can estimate

$$
\mathscr{F}_{K}^{p}\left(\langle T, f, t\rangle-\left(\partial \left(T\llcorner\{f<t\}-Z\llcorner\{f<t\})) \leq \mathbf{M}\left(\tilde{R}_{k}\right)+\mathbf{M}\left(\tilde{S}_{k}\right)+\mathbf{M}\left(\left\langle\tilde{S}_{k}, f, t\right\rangle\right) .\right.\right.\right.
$$

Since $\lim _{k}\left(\mathbf{M}\left(\tilde{R}_{k}\right)+\mathbf{M}\left(\tilde{S}_{k}\right)\right)=0$, it remains to show that, for a.e. $t$,

$$
\lim _{k \rightarrow \infty} \mathbf{M}\left(\left\langle\tilde{S}_{k}, f, t\right\rangle\right)=0 .
$$

In order to see this, fix $\varepsilon>0$. By [Sim83, Lemma 28.5(1)], we have that there is a Borel set $E_{k}$ with measure $\left|E_{k}\right| \leq \frac{\varepsilon}{2^{k}}$ such that

$$
\mathbf{M}\left(\left\langle\tilde{S}_{k}, f, t\right\rangle\right) \leq \operatorname{Lip}(f) \frac{2^{k}}{\varepsilon} \mathbf{M}\left(\tilde{S}_{k}\right) \quad \text { for all } t \notin E_{k} .
$$

In particular, if we set $E:=\bigcup_{k} E_{k}$, we have $|E| \leq 2 \varepsilon$, and using (6.10) we see that

$$
\mathbf{M}\left(\left\langle\tilde{S}_{k}, f, t\right\rangle\right) \leq \varepsilon^{-1} \operatorname{Lip}(f) k^{-1} \quad \text { for all } t \notin E .
$$

Hence $\lim _{k \rightarrow \infty} \mathbf{M}\left(\left\langle\tilde{S}_{k}, f, t\right\rangle\right)=0$ for all $t \notin E$. Since $\varepsilon$ is arbitrary, this concludes the proof. 
REMARK 6.3. We are actually able to give a much shorter proof of Lemma 6.1(i), provided one can prove that there exists an integral current $\tilde{T}$ such that $\tilde{T}=$ $T \bmod (p)$. Indeed, in this case, since $\tilde{T}$ is integral the classical slicing formula gives

$$
\langle\tilde{T}, f, t\rangle=\partial(\tilde{T}\llcorner\{f<t\})-(\partial \tilde{T})\llcorner\{f<t\} .
$$

On the other hand, the conditions $\tilde{T}=T \bmod (p)$ and $\partial \tilde{T}=\partial T=Z \bmod (p)$ imply that there are rectifiable currents $R$ and $Q$ such that $T=\tilde{T}+p R$ and $Z=\partial \tilde{T}+p Q$, and thus we deduce

$$
\langle T, f, t\rangle=\partial(T\llcorner\{f<t\})-Z\llcorner\{f<t\}+p(-\partial(R\llcorner\{f<t\})+\langle R, f, t\rangle+Q\llcorner\{f<t\}),
$$

as we wanted.

The existence of an integral representative in any integral class $\bmod (p)$ is in fact a very delicate question. If $K$ is any given compact subset of $\mathbb{R}^{m+n}$ then a class $[T] \in \mathscr{I}_{m}^{p}(K)$ does not necessarily have a representative in $\mathscr{I}_{m}(K)$ when $m \geq 2$; see [MS18, Proposition 4.10]. Positive answers have been given, instead, when $m=1$ in the class $\mathscr{I}_{m}(K)$ for any given compact $K$ in [MS18, Theorem 4.5], and in any dimension in the class $\bigcup_{K} \mathscr{I}_{m}(K)$ in the remarkable work [You18].

\section{Monotonicity formula and tangent cones}

From Lemma 5.1 and the classical monotonicity formula for stationary varifolds, cf. [All72] and [Sim83], we conclude directly the following corollary.

Corollary 7.1. Let $T, \Sigma$ and $\Omega=\mathbf{B}_{R}$ be as in Assumption 4.1. Then, if $q \in$ $\operatorname{spt}(T) \cap \Omega$, the following monotonicity identity holds for every $0<s<r<R-|q|$ :

$$
\begin{aligned}
r^{-m} & \|T\|\left(\mathbf{B}_{r}(q)\right)-s^{-m}\|T\|\left(\mathbf{B}_{s}(q)\right)-\int_{\mathbf{B}_{r}(q) \backslash \mathbf{B}_{s}(q)} \frac{\left|(x-q)^{\perp}\right|^{2}}{|x-q|^{m+2}} d\|T\|(x) \\
= & \int_{s}^{r} \rho^{-m-1} \int_{\mathbf{B}_{\rho}(q)}(x-q)^{\perp} \cdot \vec{H}_{T}(x) d\|T\|(x) d \rho,
\end{aligned}
$$

where $Y^{\perp}(x)$ denotes the component of the vector $Y(x)$ orthogonal to the tangent plane of $T$ at $x$ (which is oriented by $\vec{T}(x)$ ). In particular:

(i) There is a dimensional constant $C(m)$ such that the map $r \rightarrow e^{C\left\|A_{\Sigma}\right\|_{0} r} \frac{\|T\|\left(\mathbf{B}_{r}(q)\right)}{\omega_{m} r^{m}}$ is monotone increasing.

(ii) The limit

$$
\Theta_{T}(q):=\lim _{r \downarrow 0} \frac{\|T\|\left(\mathbf{B}_{r}(q)\right)}{\omega_{m} r^{m}}
$$

exists and is finite at every point $q \in \mathbf{B}_{R}$.

(iii) The map $q \mapsto \Theta_{T}(q)$ is upper semicontinuous and it is a positive integer at $\mathcal{H}^{m}$-a.e. $q \in \operatorname{spt}(T)$. In particular $\operatorname{spt}(T) \cap \mathbf{B}_{R}=\left\{\Theta_{T} \geq 1\right\}$. 
Next, we introduce the usual blow-up procedure to analyze tangent cones at $q \in \operatorname{spt}(T)$.

Definition 7.2. Fix a point $q \in \operatorname{spt}(T)$ and define

$$
\iota_{q, r}(x):=\frac{x-q}{r} \quad \forall r>0 .
$$

We denote by $T_{q, r}$ the currents

$$
T_{q, r}:=\left(\iota_{q, r}\right)_{\sharp} T \quad \forall r>0 .
$$

Recalling Allard's theory of stationary varifolds, we then know that, for every sequence $r_{k} \downarrow 0$, a subsequence, not relabeled, of $\mathbf{v}\left(T_{q, r_{k}}\right)$ converges locally to a varifold $C$ which is a stationary cone in $T_{q} \Sigma$ (the tangent space to $\Sigma$ at $q$ ). Combined with Proposition 5.2 we achieve the following corollary.

Corollary 7.3. Let $T, \Sigma$ and $\Omega=\mathbf{B}_{R}$ be as in Assumption 4.1, let $q \in \operatorname{spt}(T) \cap \Omega$, and let $r_{k} \downarrow 0$. Then there is a subsequence, not relabeled, and a current $T_{0}$ with the following properties:

(i) $T_{0}\left\llcorner\mathbf{B}_{\rho} \in \mathscr{R}_{m}\left(T_{q} \Sigma\right), \partial T_{0}\left\llcorner\mathbf{B}_{\rho}=0 \bmod (p)\right.\right.$ for every $\rho>0$.

(ii) $T_{0}\left\llcorner\mathbf{B}_{\rho}\right.$ is a representative $\bmod (p)$ and is area minimizing $\bmod (p)$ in $\mathbf{B}_{\rho} \cap T_{q} \Sigma$ for every $\rho>0$.

(iii) $T_{0}$ is a cone, namely $\left(\iota_{0, r}\right)_{\sharp} T_{0}=T_{0}$ for every $r>0$.

(iv) For every $\rho>0$ there is $r \geq \rho$ and $K \supset \mathbf{B}_{r}$ such that

$$
\lim _{k \rightarrow \infty} \mathscr{F}_{K}^{p}\left(T _ { q , r _ { k } } \left\llcorner\mathbf{B}_{r}-T_{0}\left\llcorner\mathbf{B}_{r}\right)=0 .\right.\right.
$$

(v) If $\operatorname{spt}^{p}\left(T_{0}\right)=\operatorname{spt}\left(T_{0}\right)$ is contained in an $m$-dimensional plane $\pi_{0}$, then $T_{0}=$ $Q \llbracket \pi_{0} \rrbracket$ for some $Q \in \mathbb{Z} \cap\left[-\frac{p}{2}, \frac{p}{2}\right]$.

Before coming to its proof, let us state an important lemma which will be used frequently during the rest of the paper. See [DPH14, Theorem 7.6] for a proof.

Lemma 7.4 (Constancy Lemma). Assume $\pi \subset \mathbb{R}^{m+n}$ is an $m$-dimensional plane and let $\Omega \subset \mathbb{R}^{m+n}$ be an open set such that $\Omega \cap \pi$ is connected. Assume $T \in \mathscr{R}_{m}(\pi)$ is a current such that $\left(\partial^{p} T\right)\left\llcorner\Omega=0\right.$. Finally let $\vec{v}=v_{1} \wedge \ldots \wedge v_{m}$ for an orthonormal basis $v_{1}, \ldots, v_{m}$ of $\pi$. Then there is a $Q \in \mathbb{Z} \cap\left[-\frac{p}{2}, \frac{p}{2}\right]$ such that $T\left\llcorner\Omega=Q \vec{v} \mathcal{H}^{m}\llcorner(\Omega \cap \pi)\right.$ $\bmod (p)$.

Proof of Corollary 7.3. Note that (v) is an obvious consequence of the constancy lemma and of (i). In order to prove the remaining statements, first extract a subsequence such that $V_{k}=\mathbf{v}\left(T_{q, r_{k}}\right)$ converges to a stationary cone $C$ as above. Then observe that for every $j \in \mathbb{N}$, using a classical Fubini argument and Lemma 6.1 we find a radius $\rho(j) \in[j, j+1]$ such that

$$
\begin{aligned}
\liminf _{k} \mathbf{M}^{p}\left(\partial\left(T_{q, r_{k}}\left\llcorner\mathbf{B}_{\rho(j)}\right)\right)\right. & =\liminf _{k} \mathbf{M}\left(\left\langle T_{q, r_{k}},|\cdot|, \rho(j)\right\rangle\right) \\
& \leq \liminf _{k}\left\|T_{q, r_{k}}\right\|\left(\mathbf{B}_{j+1} \backslash \mathbf{B}_{j}\right)=\omega_{m} \Theta_{T}(q)\left((j+1)^{m}-j^{m}\right) .
\end{aligned}
$$


Thus we can find a subsequence to which we can apply the compactness Proposition 5.2. By a standard diagonal argument we can thus find a single subsequence $r_{k}$ with the following properties:

(a) For each $j$ there is a current $T^{j} \in \mathscr{R}_{m}\left(T_{q} \Sigma\right)$ such that

$$
\lim _{k \rightarrow \infty} \mathscr{F}_{\mathbf{B}_{j+1}}^{p}\left(T_{q, r_{k}}\left\llcorner\mathbf{B}_{\rho(j)}-T^{j}\right)=0 .\right.
$$

(b) Each $T^{j}$ is a representative $\bmod (p)$ and $\mathbf{v}\left(T^{j}\right)=C\left\llcorner\mathbf{B}_{\rho(j)}\right.$.

(c) Each $T^{j}$ is area minimizing $\bmod (p)$ in $\mathbf{B}_{\rho(j)}$.

Notice next that $T^{j}\left\llcorner\mathbf{B}_{\rho(i)}=T^{i} \bmod (p)\right.$ for every $i \leq j$. If we then define the current

$$
T_{0}:=\sum_{i \in \mathbb{N}} T^{i}\left\llcorner\left(\mathbf{B}_{\rho(i)} \backslash \mathbf{B}_{\rho(i-1)}\right),\right.
$$

with $\rho(-1):=0$, then the latter satisfies the conclusions (i), (ii) and (iv).

In the remaining part of the proof we wish to show (iii), after possibly changing $T_{0}$ to another representative $\bmod (p)$ of the same class.

To this aim, consider that, by standard regularity theory for stationary varifolds, the closed set $R=\operatorname{spt}(C)$ is countably $m$-rectifiable, it is a cone with vertex at the origin and $\|C\|=\Theta_{C}(x) \mathcal{H}^{m}\left\llcorner R\right.$, where $\Theta_{C}$ is the density of the varifold $C$. By the monotonicity formula and $\mathbf{v}(T)=C$ we have

$$
\Theta_{T_{0}}(x)=\Theta_{C}(x) .
$$

If $x$ is a point where the approximate tangent $T_{x} R$ exists, we then conclude easily that, up to subsequences, we can apply the same argument above and find that $\left(T_{0}\right)_{x, r_{k}}$ with $r_{k} \downarrow 0$ converges locally $\bmod (p)$ to a current $S$ satisfying the corresponding conclusions:

(i)' $S\left\llcorner\mathbf{B}_{\rho} \in \mathscr{R}_{m}\left(T_{q} \Sigma\right)\right.$ and $\partial S\left\llcorner\mathbf{B}_{\rho}=0 \bmod (p)\right.$ for every $\rho>0$.

(ii)' $S\left\llcorner\mathbf{B}_{\rho}\right.$ is a representative $\bmod (p)$ and is area minimizing $\bmod (p)$ in $\mathbf{B}_{\rho} \cap T_{q} \Sigma$ for every $\rho>0$.

(iv)' For every $\rho>0$ there is $r \geq \rho$ and $K \supset \mathbf{B}_{r}$ such that

$$
\lim _{k \rightarrow \infty} \mathscr{F}_{K}^{p}\left(( T _ { 0 } ) _ { x , r _ { k } } \left\llcorner\mathbf{B}_{r}-S\left\llcorner\mathbf{B}_{r}\right)=0 .\right.\right.
$$

However, for $S$ we would additionally know that it is supported in $T_{x} R$, which is an $m$-dimensional plane. We then could apply the Constancy Lemma and conclude that, if $v_{1}, \ldots, v_{m}$ is an orthonormal basis of $T_{x} R$, then $\Theta_{C}(x) \in \mathbb{N} \cap\left[1, \frac{p}{2}\right]$ and, for any $\rho>0$,

$$
\begin{array}{rlr}
\text { either } S\left\llcorner\mathbf{B}_{\rho}\right. & =\Theta_{C}(x) v_{1} \wedge \ldots \wedge v_{m} \mathcal{H}^{m}\left\llcorner T_{x} R \cap \mathbf{B}_{\rho} \quad \bmod (p)\right. \\
\text { or } S\left\llcorner\mathbf{B}_{\rho}\right. & =-\Theta_{C}(x) v_{1} \wedge \ldots \wedge v_{m} \mathcal{H}^{m}\left\llcorner T_{x} R \cap \mathbf{B}_{\rho}\right. & \bmod (p) .
\end{array}
$$


In particular we conclude that there is a Borel function $\varepsilon: \operatorname{spt}(C)=R \rightarrow\{-1,1\}$ such that

$$
T_{0}=\varepsilon \Theta_{C} \vec{v} \mathcal{H}^{m}\llcorner R,
$$

where $\vec{v}(x)$ is an orienting Borel unit $m$-vector for $T_{x} R$. Clearly, since $R$ is a cone, we can choose $\vec{v}(x)$ with the additional property that $\vec{v}(x)=\vec{v}(\lambda x)$ for every positive $\lambda$. Also, since the varifold $C$ is a cone, the density $\Theta_{C}$ is 0-homogeneous as well. Moreover, at all points $x$ where $\Theta_{C}(x)=\frac{p}{2}$ we can arbitrarily set $\varepsilon(x)=1$, since this would neither change the class $\bmod (p)$, nor the fact that $T_{0}$ is representative $\bmod (p)$.

Fix now a radius $s>0$ such that the conclusions of Lemma 6.1 hold with $T=T_{0}, f=|\cdot|$, and $t=s$, and consider the cone $T^{\prime}:=\left\langle T_{0},|\cdot|, s\right\rangle \nVdash\{0\}$. Observe that $\partial\left(T^{\prime}-T_{0}\left\llcorner\mathbf{B}_{s}\right)=0 \bmod (p)\right.$. We now make the following simple observation: if $Z \in \mathscr{R}_{m}\left(\mathbb{R}^{m+n}\right)$ with $\operatorname{spt}(Z)$ compact is such that $\partial Z=0 \bmod (p)$ in $\mathbb{R}^{m+n}$, then

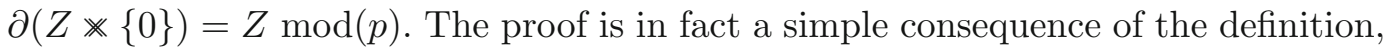
since $\partial Z=0 \bmod (p)$ implies the existence of integer rectifiable currents $Q_{k}^{(1)}$ and $Q_{k}^{(2)}$ and flat currents $Q_{k}$ such that

$$
\partial Z=p Q_{k}+Q_{k}^{(1)}+\partial Q_{k}^{(2)}
$$

and

$$
\mathbf{M}\left(Q_{k}^{(1)}\right)+\mathbf{M}\left(Q_{k}^{(2)}\right) \rightarrow 0 .
$$

Using the general formula $\partial(A \circledast 0)=A-(\partial A) ※ 0$ we then obtain

$$
\partial\left(Z \aleph_{0}\right)=Z-p Q_{k} \aleph_{0}-Q_{k}^{(1)} \mathbb{x}\{0\}+\partial\left(Q_{k}^{(2)} \aleph_{0}\right)-Q_{k}^{(2)},
$$

which by

$$
\mathbf{M}\left(Q_{k}^{(1)} \nVdash\{0\}+Q_{k}^{(2)}\right)+\mathbf{M}\left(Q_{k}^{(2)} \nVdash 0\right) \rightarrow 0
$$

implies that indeed $\partial(Z \circledast 0)=Z \bmod (p)$.

We apply the above observation to $Z=T^{\prime}-T_{0}\left\llcorner\mathbf{B}_{s}\right.$. In that case we conclude however that the cone

$$
Z * 0 \quad \text { is identically } 0
$$

because it is an $(m+1)$-dimensional rectifiable current supported in the countably $m$-rectifiable set $R$. We thus must necessarily have that $T^{\prime}-T_{0}\left\llcorner\mathbf{B}_{s}=0 \bmod (p)\right.$. Applying the argument of the previous paragraph, we of course again conclude that

$$
T^{\prime}=\varepsilon^{\prime} \Theta_{C} \vec{v} \mathcal{H}^{m}\left\llcorner R \cap \mathbf{B}_{s} .\right.
$$

Consider now, as above, a point $x \in \mathbf{B}_{s}$ where the approximate tangent plane to $R$ exists. Then $\left(T^{\prime}\right)_{x, r}$ converges, as $r \downarrow 0$, to $\varepsilon^{\prime}(x) \Theta_{C}(x) \vec{v}(x) \mathcal{H}^{m}\left\llcorner T_{x} R\right.$, whereas $\left(T_{0}\right)_{x, r}$ converges, as $r \downarrow 0$, to $\varepsilon(x) \Theta_{C}(x) \vec{v}(x) \mathcal{H}^{m}\left\llcorner T_{x} R\right.$. However the two limits must be congruent $\bmod (p)$ and, in case $\Theta_{C}(x)<\frac{p}{2}$, this necessarily implies $\varepsilon(x)=\varepsilon^{\prime}(x)$. 
Fix now $\lambda>0$. Since $T^{\prime}$ is a cone and $s$ is arbitrary, we conclude that for $\mathcal{H}^{m}$ a.e. $x \in R \cap\left\{\Theta_{C}<\frac{p}{2}\right\}$ we must necessarily have $\varepsilon(x)=\varepsilon^{\prime}(x)=\varepsilon^{\prime}(\lambda x)=\varepsilon(\lambda x)$. On the other hand we already have $\varepsilon(x)=\varepsilon(\lambda x)=1$ if $\Theta_{C}(x)=\frac{p}{2}$. Hence we have concluded that $\varepsilon(\lambda x)=\varepsilon(x)$ for $\mathcal{H}^{m}$-a.e. $x \in R$. In particular $\left(\iota_{0, \lambda}\right)_{\sharp} T_{0}=T_{0}$. The arbitrariness of $\lambda$ implies now the desired conclusion (iii) and completes the proof of the corollary.

\section{Strata and blow-up sequence}

Definition 8.1. ( $Q$-points) For every $Q \in \mathbb{N} \backslash\{0\}$, we will let $\mathrm{D}_{Q}(T)$ denote the points of density $Q$ of the current $T$, namely

$$
\mathrm{D}_{Q}(T):=\left\{q \in \Omega: \Theta_{T}(q)=Q\right\}
$$

We also set

$$
\operatorname{Reg}_{Q}(T):=\operatorname{Reg}(T) \cap \mathrm{D}_{Q}(T) \quad \text { and } \quad \operatorname{Sing}_{Q}(T):=\operatorname{Sing}(T) \cap \mathrm{D}_{Q}(T) .
$$

Theorem 1.7 is thus equivalent to

Theorem 8.2. Under Assumption 4.1, for every $Q<\frac{p}{2}$ the set $\operatorname{Sing}_{Q}(T)$ has Hausdorff dimension at most $m-2$.

Before proceeding, we need to recall the following definition.

Definition 8.3. An integral $m$-varifold $V$ is called a $k$-symmetric cone (where $0 \leq$ $k \leq m$ ) if it can be written as the product of a $k$-dimensional plane passing through the origin times an $(m-k)$-dimensional cone. The largest plane passing through the origin such that the above holds is called the spine of $V$. If $V$ is stationary, then the standard stratification of $V$ is

$$
\mathcal{S}^{0} \subset \mathcal{S}^{1} \subset \cdots \subset \mathcal{S}^{m}
$$

where

$$
\mathcal{S}^{k}:=\{q \in \operatorname{spt}(V): \text { no tangent cone to } V \text { at } q \text { is }(k+1) \text {-symmetric }\} .
$$

As a consequence of Corollary 7.3 and of the classical Almgren's stratification theorem, we have now the following

Proposition 8.4. Let T, $\Sigma$ and $\Omega$ be as in Assumption 4.1 and consider the set

$$
Z:=\Omega \cap \operatorname{spt}(T) \backslash \bigcup_{Q \in \mathbb{N} \backslash\{0\}, Q \leq \frac{p}{2}} \mathrm{D}_{Q}(T) .
$$

Then $\mathcal{H}^{m-1+\alpha}(Z)=0$ for every $\alpha>0$. 
Proof. By Lemma 5.1, the varifold $V=\mathbf{v}(T)$ is stationary in $\Sigma \cap \Omega$, thus we can consider the stratification of $V$ as in (8.1) and (8.2). If $q \in \mathcal{S}^{m} \backslash \mathcal{S}^{m-1}$ then there is at least one tangent cone to $V$ at $q$ which is supported in a flat plane $\pi_{0}$. Then there is a current $T_{0}$ as in Corollary 7.3, obtained as a limit $T_{q, r_{k}}$ for an appropriate $r_{k} \downarrow 0$, which satisfies $\mathbf{v}\left(T_{0}\right)=V$. Thus by the constancy lemma $\Theta_{T_{0}}(0)=\Theta_{T}(q)$ must belong to $\left[1, \frac{p}{2}\right] \cap \mathbb{N}$. This implies that $Z \subset \mathcal{S}^{m-1}$. Our statement then follows immediately from the well known fact that $\operatorname{dim}_{\mathcal{H}} \mathcal{S}^{k} \leq k$ for every $0 \leq k \leq m$.

We shall also need the following elementary yet fundamental lemmas. Given $v \in \mathbb{R}^{m+n}$, we will adopt the notation $\tau_{v}:=\iota_{v, 1}$, so that $\tau_{v}(x):=x-v$.

Lemma 8.5. Assume $T \in \mathscr{R}_{m}\left(\mathbb{R}^{m+n}\right)$ is an $m$-dimensional integer rectifiable current such that $\partial T=0 \bmod (p)$ and the associated varifold $\mathbf{v}(T)$ is a $k$-symmetric cone with spine $\mathbb{R}^{k} \times\{0\} \subset \mathbb{R}^{m+n}$. Then

$$
\left(\tau_{v}\right)_{\sharp} T=T \bmod (p) \quad \text { for every } v \in \mathbb{R}^{k} \times\{0\},
$$

and there exists an $(m-k)$-dimensional cone $T^{\prime}$ such that

$$
T=\llbracket \mathbb{R}^{k} \rrbracket \times T^{\prime} \bmod (p) .
$$

Furthermore, if $T$ is a representative $\bmod (p)$ then so is $T^{\prime}$; in this case, $\mathbf{v}(T)=$ $\mathbf{v}\left(\llbracket \mathbb{R}^{k} \rrbracket \times T^{\prime}\right)$, and $\mathbf{v}\left(T^{\prime}\right)$ has trivial spine. Finally, if $T$ is locally area minimizing $\bmod (p)$, then so is $T^{\prime}$.

Proof. Write $T=\llbracket M, \vec{\tau}, \theta \rrbracket$, so that $\mathbf{v}(T)=\mathbf{v}(M,|\theta|)$. Since $\mathbf{v}(T)$ is a $k$-symmetric cone with spine $\mathbb{R}^{k} \times\{0\}$, the locally $\mathcal{H}^{m}$-rectifiable set $M$ is a cone which is invariant with respect to $\mathbb{R}^{k} \times\{0\}$, in the sense that there exists a locally $\mathcal{H}^{m-k}$-rectifiable set $M^{\prime} \subset \mathbb{R}^{m+n-k}$ such that $M=\mathbb{R}^{k} \times M^{\prime}$. Furthermore, $|\theta|$ is a 0-homogeneous function such that $|\theta|(x+v)=|\theta|(x)$ for every $v \in \mathbb{R}^{k} \times\{0\}$. By the properties of $M$, modulo changing the sign of $\theta$, we can also assume that the orienting unit $m$-vector field $\vec{\tau}$ is a 0 -homogeneous function such that $\vec{\tau}(x+v)=\vec{\tau}(x)$ for every $v \in \mathbb{R}^{k} \times\{0\}$.

Now, given two smooth and proper maps $f, g: \mathbb{R}^{m+n} \rightarrow \mathbb{R}^{m+n}$, and letting $h:[0,1] \times \mathbb{R}^{m+n} \rightarrow \mathbb{R}^{m+n}$ be the linear homotopy from $f$ to $g$, namely the function defined by

$$
h(t, x):=(1-t) f(x)+\operatorname{tg}(x),
$$

the homotopy formula (see [Sim83, Equation 26.22]) states that

$$
g_{\sharp} T-f_{\sharp} T=\partial h_{\sharp}(\llbracket(0,1) \rrbracket \times T)+h_{\sharp}(\llbracket(0,1) \rrbracket \times \partial T) .
$$

Since $\partial T=0 \bmod (p),(8.5)$ yields

$$
g_{\sharp} T-f_{\sharp} T=\partial h_{\sharp}(\llbracket(0,1) \rrbracket \times T) \quad \bmod (p) .
$$

Now, let $v \in \mathbb{R}^{k} \times\{0\}$, and apply (8.6) with

$$
f(x)=x \quad \text { and } \quad g(x)=\tau_{v}(x)=x-v .
$$


We can compute, for any $\omega \in \mathscr{D}_{1+m}\left(\mathbb{R} \times \mathbb{R}^{m+n}\right)$ :

$$
\begin{aligned}
h_{\sharp}(\llbracket(0,1) \rrbracket \times T)(\omega): & =(\llbracket(0,1) \rrbracket \times T)\left(h^{\sharp} \omega\right) \\
& =\int_{0}^{1} d t \int\left\langle\omega(h(t, x)),\left[d h_{(t, x)}\right]_{\sharp}\left(e_{1} \wedge \vec{T}(x)\right)\right\rangle d\|T\|(x) \\
& =-\int_{0}^{1} d t \int\langle\omega(h(t, x)), v \wedge \vec{T}(x)\rangle d\|T\|(x)=0,
\end{aligned}
$$

where we have used that $v \in \mathbb{R}^{k} \times\{0\}, \vec{T}(x) \in \Lambda_{m}(\operatorname{Tan}(M, x))$ at $\|T\|$-a.e. $x$, and $M$ is invariant with respect to $\mathbb{R}^{k} \times\{0\}$. Using that $\omega$ can be chosen arbitrarily, we conclude (8.3) from (8.6).

Next, let $\mathbf{p}: \mathbb{R}^{m+n} \rightarrow \mathbb{R}^{m+n}$ be the orthogonal projection operator onto $\mathbb{R}^{k} \times\{0\}$. Using standard properties of the slicing of integer rectifiable currents (see e.g. [Fed69, Theorem 4.3.2(7)]) and (8.3), we can conclude then that

$$
\left(\tau_{v}\right)_{\sharp}\langle T, \mathbf{p}, z+v\rangle=\left\langle\left(\tau_{v}\right)_{\sharp} T, \mathbf{p}, z\right\rangle=\langle T, \mathbf{p}, z\rangle \bmod (p),
$$

for every $z, v \in \mathbb{R}^{k} \times\{0\}$ such that the slices exist, or, equivalently, that

$$
\langle T, \mathbf{p}, z\rangle=\left(\tau_{w-z}\right)_{\sharp}\langle T, \mathbf{p}, w\rangle \bmod (p)
$$

for every $z, w \in \mathbb{R}^{k} \times\{0\}$ such that the slices exist. Fix $z$ such that $\langle T, \mathbf{p}, z\rangle$ exists, and let $T^{\prime} \in \mathscr{R}_{m-k}\left(\mathbb{R}^{m+n-k}\right)$ be such that $\langle T, \mathbf{p}, z\rangle=\left(\tau_{-z}\right)_{\sharp} T^{\prime}$ after identifying $\mathbb{R}^{m+n-k}$ with $\{0\} \times \mathbb{R}^{m+n-k}$. Then, the current $\tilde{T}:=\llbracket \mathbb{R}^{k} \rrbracket \times T^{\prime}$ satisfies

$$
\langle T-\tilde{T}, \mathbf{p}, z\rangle=0 \quad \bmod (p) \quad \text { for } \mathcal{H}^{k} \text {-a.e. } z \in \mathbb{R}^{k} \times\{0\} .
$$

Observe that we may write

$$
T=\theta \vec{\tau} \mathcal{H}^{m}\left\llcorner M, \quad \tilde{T}=\tilde{\theta} \vec{\tau} \mathcal{H}^{m}\llcorner M,\right.
$$

for a 0 -homogeneous function $\tilde{\theta}$ such that $\tilde{\theta}(x+v)=\tilde{\theta}(x)$ for every $v \in \mathbb{R}^{k} \times\{0\}$. Also notice that, since $M$ is invariant with respect to $\mathbb{R}^{k} \times\{0\}$ and $\mathbf{p}$ is the orthogonal projection onto $\mathbb{R}^{k} \times\{0\}$, if we identify $\mathbb{R}^{k} \times\{0\}$ with $\mathbb{R}^{k}$ and if we set $\phi:=\left.\mathbf{p}\right|_{M}$, then $J_{k} \phi(x)>0$ for $\mathcal{H}^{m}$-a.e. $x \in M$, where $J_{k} \phi(x)$ is the $k$-dimensional Jacobian of $\phi$, defined by

$$
J_{k} \phi(x):=\left(\operatorname{det}\left(d \phi(x) \circ d \phi(x)^{T}\right)\right)^{1 / 2}, \quad d \phi(x): T_{x} M \rightarrow \mathbb{R}^{k}
$$

at all points $x \in M$ such that $T_{x} M$ exists.

By the considerations above, the standard slicing theory of rectifiable currents (see e.g. [Fed69, Theorem 4.3.8]) implies that for $\mathcal{H}^{k}$-a.e. $z \in \mathbb{R}^{k} \times\{0\}$ the set $M_{z}:=M \cap \mathbf{p}^{-1}(z)$ is $(m-k)$-rectifiable and

$$
\langle T, \mathbf{p}, z\rangle=\llbracket M_{z}, \zeta,\left.\theta\right|_{M_{z}} \rrbracket, \quad\langle\tilde{T}, \mathbf{p}, z\rangle=\llbracket M_{z}, \zeta,\left.\tilde{\theta}\right|_{M_{z}} \rrbracket
$$


for a Borel measurable unit $(m-k)$-vector field $\zeta=\zeta_{z}$ which is uniquely determined by $\vec{\tau}$ and $d \phi$. If $z \in \mathbb{R}^{k} \times\{0\}$ is such that both (8.9) and (8.11) hold, then

$$
\theta(x)=\tilde{\theta}(x) \quad \bmod (p) \quad \text { at } \mathcal{H}^{m-k} \text {-a.e. } x \in M_{z} .
$$

By Fubini's theorem, the conclusion in (8.12) holds at $\mathcal{H}^{m}$-a.e. $x \in M$, so that $(8.4)$ follows from (8.10) and the definition of $\tilde{T}$.

If $T$ is a representative $\bmod (p)$, then $\langle T, \mathbf{p}, z\rangle$ is a representative $\bmod (p)$ for $\mathcal{H}^{k}$ a.e. $z \in \mathbb{R}^{k} \times\{0\}$, and thus we can choose $z$ such that the corresponding $T^{\prime}$ is a representative $\bmod (p)$. With this choice, $\tilde{T}$ is a representative $\bmod (p)$ as well, and since $\tilde{\theta}(x)=\theta(x) \bmod (p)$ for $\mathcal{H}^{m}$-a.e. $x \in M$ we deduce that

$$
\tilde{\theta}(x)=\varepsilon(x) \theta(x) \quad \text { with } \varepsilon(x) \in\{-1,1\} \text {, for } \mathcal{H}^{m} \text {-a.e. } x \in M,
$$

where $\varepsilon(x)=1$ or $|\theta(x)|=\frac{p}{2}$. As a consequence, $|\tilde{\theta}|=|\theta| \mathcal{H}^{m}\llcorner M$-a.e., which in turn implies that $\mathbf{v}(\tilde{T})=\mathbf{v}(T)$. The last conclusion of the lemma is elementary, and the details of the proof are omitted.

Lemma 8.6. Assume $T_{0} \in \mathscr{R}_{m}\left(\mathbb{R}^{m+n}\right)$ is an $m$-dimensional locally area minimizing current $\bmod (p)$ without boundary $\bmod (p)$ which is a cone (in the sense of Corollary 7.3 (iii)). Suppose, furthermore, that $\mathbf{v}\left(T_{0}\right)$ is $(m-1)$-symmetric but not $m$ symmetric (namely not flat). Then, $\Theta\left(T_{0}, 0\right) \geq \frac{p}{2}$.

Proof. Let $T_{0}=\llbracket M, \vec{\tau}, \theta \rrbracket$, so that $\mathbf{v}\left(T_{0}\right)=\mathbf{v}(M,|\theta|)$. Since $\mathbf{v}\left(T_{0}\right)$ is $(m-1)$ symmetric but not $m$-symmetric, by Lemma $8.5 T_{0}=\llbracket \pi \rrbracket \times T_{0}^{\prime} \bmod (p)$, where $\pi$ is the $(m-1)$-dimensional spine of $\mathbf{v}\left(T_{0}\right)$, and $T_{0}^{\prime}$ is a one-dimensional cone which has no $\operatorname{boundary} \bmod (p)$ and is locally area minimizing $\bmod (p)$. Since $\Theta\left(T_{0}^{\prime}, 0\right)=\Theta\left(T_{0}, 0\right)$, we can reduce the proof of the lemma to the case when $m=1$.

Thus we can assume that $T_{0}=\sum_{i} Q_{i} \llbracket \ell_{i} \rrbracket$, where $\ell_{1}, \ldots, \ell_{N}$ are pairwise distinct oriented half lines in $\mathbb{R}^{1+n}$ with the origin as common endpoint and the $Q_{i}$ 's are integers. Without loss of generality we can assume that $\partial \llbracket \ell_{i} \rrbracket=-\llbracket 0 \rrbracket$. Observe that

$$
\Theta\left(T_{0}, 0\right)=\frac{1}{2} \sum_{i}\left|Q_{i}\right|
$$

and that $\sum_{i} Q_{i}=0 \bmod (p)$ since $T_{0}$ has no boundary $\bmod (p)$. If $\sum_{i} Q_{i}=0$, then $T_{0}$ would be an integral current without boundary, which in turn would have to be area minimizing. But since $T_{0}$ is by assumption not flat, this is not possible. Thus $\sum_{i} Q_{i}=k p$ for some nonzero integer $k$. This clearly implies

$$
\sum_{i}\left|Q_{i}\right| \geq|k| p \geq p
$$

which in turn yields $\Theta\left(T_{0}, 0\right) \geq \frac{p}{2}$. 
We are now ready to state the starting point of our proof of Theorem 4.3 and Theorem 8.2, which will be achieved by contradiction.

Proposition 8.7 (Contradiction sequence). Assume Theorem 8.2 is false. Then there are integers $m, n \geq 1$ and $2 \leq Q<\frac{p}{2}$ and reals $\alpha, \eta>0$ with the following property. There are

(i) $T, \Sigma$ and $\Omega$ as in Assumption 4.1 such that $0 \in \operatorname{Sing}_{Q}(T)$;

(ii) a sequence of radii $r_{k} \downarrow 0$ and an m-dimensional plane $\pi_{0}$ such that $\mathbf{v}\left(T_{0, r_{k}}\right)$ converges to $V=Q \mathcal{H}^{m}\left\llcorner\pi_{0} \otimes \delta_{\pi_{0}}\right.$;

(iii) $\lim _{k \rightarrow \infty} \mathcal{H}_{\infty}^{m-2+\alpha}\left(\mathrm{D}_{Q}\left(T_{0, r_{k}}\right) \cap \mathbf{B}_{1}\right) \geq \eta$.

If Theorem 4.3 is false then either there is a sequence as above or, for $Q=\frac{p}{2}$, there is a sequence as above where (iii) is replaced by

(iii) $\lim _{k \rightarrow \infty} \mathcal{H}_{\infty}^{m-1+\alpha}\left(\mathrm{D}_{Q}\left(T_{0, r_{k}}\right) \cap \mathbf{B}_{1}\right) \geq \eta$.

Proof. Suppose first that Theorem 4.3 is false. Fix $p \in \mathbb{N} \backslash\{0,1\}$, and let $m \geq 1$ be the smallest integer for which the assertion of Theorem 4.3 is false. Observe that $m>1$. Fix thus a $T, \Sigma$ and $\Omega$ satisfying Assumption 4.1 for which there is an $\alpha>0$ with $\mathcal{H}^{m-1+\alpha}(\operatorname{Sing}(T))>0$. Then, by Proposition 8.4, there must be a $Q \in \mathbb{N} \cap\left[1, \frac{p}{2}\right]$ such that $\mathcal{H}^{m-1+\alpha}\left(\operatorname{Sing}_{Q}(T)\right)>0$.

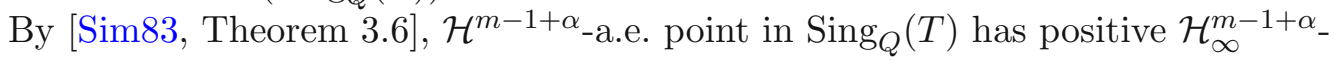
upper density: fix a point $q$ with this property, and assume, without loss of generality, that $q=0$ and that $(\partial T)\left\llcorner\mathbf{B}_{1}=0 \bmod (p)\right.$. Then, there exists a sequence of radii $r_{k}$ such that $r_{k} \downarrow 0$ as $k \rightarrow \infty$ and such that

$$
\lim _{k \rightarrow \infty} \mathcal{H}_{\infty}^{m-1+\alpha}\left(\operatorname{Sing}_{Q}\left(T_{0, r_{k}}\right) \cap \mathbf{B}_{1}\right)=\lim _{k \rightarrow \infty} \frac{\mathcal{H}_{\infty}^{m-1+\alpha}\left(\operatorname{Sing}_{Q}(T) \cap \mathbf{B}_{r_{k}}\right)}{r_{k}^{m-1+\alpha}}>0 .
$$

Moreover, we can assume that the sequence of stationary varifolds $\mathbf{v}\left(T_{0, r_{k}}\right)$ converges to a stationary cone $C \subset T_{0} \Sigma$. Consider the compact sets $\left\{\Theta_{T_{0, r_{k}}} \geq Q\right\} \cap \mathbf{B}_{1}$ and assume, without loss of generality, that they converge in the Hausdorff sense to a compact set $K$. As it is well known, by the monotonicity formula for stationary varifolds we must have $\Theta_{C}(q) \geq Q$ for every $q \in K$. On the other hand, this implies that every point $q \in K$ belongs to the spine of the cone $C$; see [Whi97]. In turn, by the upper semicontinuity of the $\mathcal{H}_{\infty}^{m-1+\alpha}$ measure with respect to Hausdorff convergence of compact sets, we have

$$
\mathcal{H}_{\infty}^{m-1+\alpha}(K) \geq \limsup _{k \rightarrow \infty} \mathcal{H}_{\infty}^{m-1+\alpha}\left(\mathrm{D}_{Q}\left(T_{0, r_{k}}\right) \cap \overline{\mathbf{B}}_{1}\right)>0 .
$$

Recall that the spine of the cone $C$ is however a linear subspace of $\mathbb{R}^{m+n}$, cf. again [Whi97]. This implies in turn that $C$ must be supported in a plane, which completes the proof under the assumption that Theorem 4.3 is false.

Now, let us suppose Theorem 8.2 is false. Then, we can find $p, m, n$ and $Q<\frac{p}{2}$, together with $\Omega, \Sigma, T$ as in Assumption 4.1, and $\alpha>0$ such that 
$\mathcal{H}^{m-2+\alpha}\left(\operatorname{Sing}_{Q}(T)\right)>0$. Arguing as above, we can then find a point $q \in \operatorname{Sing}_{Q}(T)$ with positive $\mathcal{H}_{\infty}^{m-2+\alpha}$-upper density, and we can suppose, without loss of generality, that $q=0$. Then, there is a sequence of radii $r_{k}$ with $r_{k} \downarrow 0$ as $k \rightarrow \infty$ such that:

- the blow-up sequence $T_{0, r_{k}}$ converges, in the sense of Corollary 7.3 (iv), to a current $T_{0} \in \mathscr{R}_{m}\left(T_{0} \Sigma\right)$ satisfying properties (i), (ii), and (iii) of Corollary 7.3;

- $\lim _{k \rightarrow \infty} \mathcal{H}_{\infty}^{m-2+\alpha}\left(\operatorname{Sing}_{Q}\left(T_{0, r_{k}}\right) \cap \mathbf{B}_{1}\right)>0$

- the sequence of varifolds $\mathbf{v}\left(T_{0, r_{k}}\right)$ converges to a stationary cone $C$ in $T_{0} \Sigma$;

- $C=\mathbf{v}\left(T_{0}\right)$.

- the spine of $C$ is a linear subspace of $T_{0} \Sigma$ having dimension at least $m-1$.

Now, if the spine of $C$ is $(m-1)$-dimensional, then $C$ is $(m-1)$-symmetric but not flat, hence forcing $\Theta\left(T_{0}, 0\right) \geq \frac{p}{2}$ by Lemma 8.6, which is a contradiction to the fact that $0 \in \mathrm{D}_{Q}(T)$ with $Q<\frac{p}{2}$. Thus, $C$ is supported in an $m$-dimensional plane, and the proof is complete.

\section{Part 2. Approximation with multiple valued graphs}

Following the blueprint of Almgren's partial regularity theory for area minimizing currents, we now wish to show that any area minimizing current modulo $p$ can be efficiently approximated, in a region where it is "sufficiently flat", with the graph of a multiple valued function which minimizes a suitably defined Dirichlet energy. Suppose that, in the region of interest, the current is a $Q$-fold cover of a given $m$-plane $\pi$, where $Q \in\left[1, \frac{p}{2}\right]$. The "classical" theory of Dir-minimizing $Q$-valued functions as in [DLS11] is powerful enough to accomplish the task whenever $Q<\frac{p}{2}$ (which is always the case when $p$ is odd). If $p$ is even and $Q=\frac{p}{2}$, on the other hand, Almgren's $Q$-valued functions are not anymore the appropriate maps, and we will need to work with the class of special multiple valued function defined in [DLHMS].

\section{First Lipschitz approximation}

From now on we denote by $B_{r}(x, \pi)$ the disk $\mathbf{B}_{r}(x) \cap(x+\pi)$, where $\pi$ is some linear $m$-dimensional plane. The symbol $\mathbf{C}_{r}(x, \pi)$, instead, will always denote the cylinder $B_{r}(x, \pi) \times \pi^{\perp}$. If we omit the plane $\pi$ we then assume that $\pi=\pi_{0}:=\mathbb{R}^{m} \times\{0\}$, and the point $x$ will be omitted when it is the origin. Let $e_{i}$ be the unit vectors in the standard basis. We will regard $\pi_{0}$ as an oriented plane and we will denote by $\vec{\pi}_{0}$ the $m$-vector $e_{1} \wedge \ldots \wedge e_{m}$ orienting it. We denote by $\mathbf{p}_{\pi}$ and $\mathbf{p}_{\pi}^{\perp}$ the orthogonal projection operators onto, respectively, $\pi$ and its orthogonal complement $\pi^{\perp}$. If we omit the subscript we then assume again that $\pi=\pi_{0}$.

We will make the following

Assumption 9.1. $\Sigma \subset \mathbb{R}^{m+n}$ is a $C^{2}$ submanifold of dimension $m+\bar{n}=m+n-l$, which is the graph of an entire function $\Psi: \mathbb{R}^{m+\bar{n}} \rightarrow \mathbb{R}^{l}$ satisfying the bounds

$$
\|D \Psi\|_{0} \leq c_{0} \quad \text { and } \quad \mathbf{A}:=\left\|A_{\Sigma}\right\|_{0} \leq c_{0},
$$


where $c_{0}$ is a positive (small) dimensional constant. $T$ is a representative $\bmod (p)$ of dimension $m$ with $\operatorname{spt}(T) \subset \Sigma$ and which, for some open cylinder $\mathbf{C}_{4 r}(x)$ (with $r \leq 1)$ and some positive integer $Q \leq \frac{p}{2}$, satisfies

$$
\mathbf{p}_{\sharp} T=Q \llbracket B_{4 r}(x) \rrbracket \bmod (p) \quad \text { and } \quad(\partial T)\left\llcorner\mathbf{C}_{4 r}(x)=0 \bmod (p) .\right.
$$

We next define the following relevant quantities.

Definition 9.2 (Excess measure). For a current $T$ as in Assumption 9.1 we define the cylindrical excess $\mathbf{E}\left(T, \mathbf{C}_{4 r}(x)\right)$, the excess measure $\mathbf{e}_{T}$ and its density $\mathbf{d}_{T}$ :

$$
\begin{aligned}
\mathbf{E}\left(T, \mathbf{C}_{4 r}(x)\right) & :=\frac{1}{\omega_{m}(4 r)^{m}}\left(\|T\|\left(\mathbf{C}_{4 r}(x)\right)-Q\left|B_{4 r}(x)\right|\right), \\
\mathbf{e}_{T}(A) & :=\|T\|\left(A \times \mathbb{R}^{n}\right)-Q|A|, \quad \text { for every Borel } A \subset B_{4 r}(x) \\
\mathbf{d}_{T}(y) & :=\limsup _{s \rightarrow 0} \frac{\mathbf{e}_{T}\left(B_{s}(y)\right)}{\omega_{m} s^{m}}=\limsup _{s \rightarrow 0} \mathbf{E}\left(T, \mathbf{C}_{s}(y)\right) .
\end{aligned}
$$

The subscript ${ }_{T}$ will be omitted whenever it is clear from the context.

We define the height function of $T$ in the cylinder $\mathbf{C}_{4 r}(x)$ by

$$
\mathbf{h}\left(T, \mathbf{C}_{4 r}(x), \pi_{0}\right):=\sup \left\{\left|\mathbf{p}^{\perp}(q)-\mathbf{p}^{\perp}\left(q^{\prime}\right)\right|: q, q^{\prime} \in \operatorname{spt}(T) \cap \mathbf{C}_{4 r}(x)\right\} .
$$

REMARK 9.3. Note that, since $T$ is a representative $\bmod (p)$, we have $\|T\|=\|T\|_{p}$, where $\|T\|_{p}$ denotes the Radon measure on $\mathbb{R}^{m+n}$ defined by the $\operatorname{mass} \bmod (p)$. However, it is false in general that $\left\|\mathbf{p}_{\sharp} T\right\|(A)=Q|A|$, since $\mathbf{p}_{\sharp} T$ is not necessarily a representative $\bmod (p)$. The excess written above can thus be rewritten as $\omega_{m}^{-1}(4 r)^{-m}\left(\|T\|_{p}\left(\mathbf{C}_{4 r}(x)\right)-\left\|\mathbf{p}_{\sharp} T\right\|_{p}\left(\mathbf{C}_{4 r}(x)\right)\right)$, but not as $\omega_{m}^{-1}(4 r)^{-m}$ $\left(\|T\|\left(\mathbf{C}_{4 r}(x)\right)-\left\|\mathbf{p}_{\sharp} T\right\|\left(\mathbf{C}_{4 r}(x)\right)\right)$, which is the standard cylindrical excess in the classical regularity theory for area minimizing currents. Of course, since $\left\|\mathbf{p}_{\sharp} T\right\|_{p} \leq\left\|\mathbf{p}_{\sharp} T\right\|$ as measures, this "excess $\bmod (p)$ " is, in general, larger than the classical excess.

Observe also that, under the assumptions valid in the regularity theory for classical area minimizing currents, where the identities in (9.2) hold in the sense of currents and not only $\bmod (p)$, the cylindrical excess can be classically written as

$$
\frac{1}{\omega_{m}(4 r)^{m}}\left(\|T\|\left(\mathbf{C}_{4 r}(x)\right)-\left\|\mathbf{p}_{\sharp} T\right\|\left(\mathbf{C}_{4 r}(x)\right)\right)=\frac{1}{2 \omega_{m}(4 r)^{m}} \int_{\mathbf{C}_{4 r}(x)}\left|\vec{T}-\overrightarrow{\pi_{0}}\right|^{2} d\|T\|,
$$

see e.g. [KP08, Lemma 9.1.5]. The quantity appearing on the right-hand side of (9.3) is the most flexible and natural in view of the forthcoming elliptic estimates. Unfortunately, in our setting not only the identity in (9.3) is false, but we do not have an integral representation of the excess $\bmod (p)$ either. For these reasons, later on we shall introduce a different notion of excess, called the nonoriented excess (see (13.2)), which shares the structural features of the quantity on the right-hand side of (9.3). The nonoriented excess and the excess $\bmod (p)$ are then shown to be comparable in appropriate smallness regimes in Theorem 16.1. Nonetheless, in the context of the Lipschitz approximation we will work with the excess $\bmod (p)$, because it is more 
suitable to the arguments involving slicing which are needed in the BV estimate of Lemma 10.3.

DeFinition 9.4. In general, given a measure $\mu$ on a domain $\Omega \subset \mathbb{R}^{m}$ we define its noncentered maximal function as

$$
\mathbf{m} \mu(y):=\sup \left\{\frac{\mu\left(B_{s}(z)\right)}{\omega_{m} s^{m}}: y \in B_{s}(z) \subset \Omega\right\} .
$$

If $f$ is a locally Lebesgue integrable non-negative function, we denote by $\mathbf{m} f$ the maximal function of the measure $f \mathscr{L}^{m}$.

The first Lipschitz approximation is given by the following proposition, according to which a representative $\bmod (p) T$ as in Assumption 9.1 can be realized as the graph of a Lipschitz continuous multiple valued function in regions where the maximal function of its excess measure is suitably small. As already motivated, the approximating function needs to be a special multi-valued function whenever $p$ is even and $Q=\frac{p}{2}$. Concerning special multi-valued functions, we will adopt the notation introduced in [DLHMS]: in particular, the space of special $Q$-points in $\mathbb{R}^{n}$ is denoted $\mathscr{A}_{Q}\left(\mathbb{R}^{n}\right), \mathcal{G}_{s}$ is the metric on it, and $|S|:=\mathcal{G}_{S}(S, Q \llbracket 0 \rrbracket)$ if $S \in \mathscr{A}_{Q}\left(\mathbb{R}^{n}\right)$. Given a function $u: \Omega \rightarrow \mathscr{A}_{Q}\left(\mathbb{R}^{n}\right)$ (possibly classical, namely with target $\mathcal{A}_{Q}\left(\mathbb{R}^{n}\right)$ ), we will let $\operatorname{Gr}(u)$ and $\mathbf{G}_{u}$ denote the set-theoretic graph of $u$ and the integer rectifiable current associated with it, respectively; see Section 2 and [DLHMS, Definition 4.1]. Also, we will let $\operatorname{osc}(u)$ denote the quantity

$$
\operatorname{osc}(u):=\inf _{q \in \mathbb{R}^{n}}\|\mid u \ominus q\|\left\|_{L^{\infty}(\Omega)}=\inf _{q \in \mathbb{R}^{n}}\right\| \mathcal{G}_{s}(u(x), Q \llbracket q \rrbracket) \|_{L^{\infty}(\Omega)} .
$$

REMARK 9.5. The definition given in (9.4) for the quantity $\operatorname{osc}(u)$ is the special multi-valued counterpart of the definition provided in [DLS14] for the $\mathcal{A}_{Q}\left(\mathbb{R}^{n}\right)$-valued case. In [DLS16a], on the other hand, the following comparable definition for the oscillation is used:

$$
\operatorname{osc}_{C}(u):=\sup \{|v-w|: x, y \in \Omega, v \in \operatorname{spt}(u(x)), w \in \operatorname{spt}(u(y))\} .
$$

More precisely one has

$$
\frac{1}{2} \operatorname{osc}_{C}(u) \leq \operatorname{osc}(u) \leq \sqrt{Q} \operatorname{osc}_{C}(u)
$$

To see the first inequality, let $x, y \in \Omega$ and $v \in \operatorname{spt}(u(x)), w \in \operatorname{spt}(u(y))$; then, for any $q \in \mathbb{R}^{n}$ we have

$$
|v-w| \leq|v-q|+|w-q| \leq|u(x) \ominus q|+|u(y) \ominus q| \leq 2\||u \ominus q|\|_{L^{\infty}(\Omega)} .
$$

Taking the infimum over all $q \in \mathbb{R}^{n}$ gives the claimed inequality. For the second inequality, fix any arbitrary $y \in \Omega$ and $q \in \operatorname{spt}(u(y))$. Then, for any $x \in \Omega$ we have

$$
|u(x) \ominus q| \leq \sqrt{Q} \operatorname{osc}_{C}(u)
$$

Taking the supremum over all $x \in \Omega$ and afterwards the infimum in $q \in \operatorname{spt}(u(y))$ gives the desired bound. 
Proposition 9.6 (Lipschitz approximation). There exists a constant $C=C$ $(m, n, Q)>0$ with the following properties. Let $T$ and $\Psi$ be as in Assumption 9.1 in the cylinder $\mathbf{C}_{4 s}(x)$. Set $E:=\mathbf{E}\left(T, \mathbf{C}_{4 s}(x)\right)$, let $0<\delta<1$ be such that $16^{m} E<\delta$, and define

$$
K:=\left\{\mathbf{m e}_{T} \leq \delta\right\} \cap B_{3 s}(x) .
$$

Then, there is a Lipschitz map $u$ defined on $B_{3 s}(x)$ and taking either values in $\mathcal{A}_{Q}\left(\mathbb{R}^{n}\right)$, if $Q<\frac{p}{2}$, or in $\mathscr{A}_{Q}\left(\mathbb{R}^{n}\right)$, if $Q=\frac{p}{2}$, for which the following facts hold.

(i) $\operatorname{Gr}(u) \subset \Sigma$;

(ii) $\operatorname{Lip}(u) \leq C\left(\delta^{1 / 2}+\|D \Psi\|_{0}\right)$ and $\operatorname{osc}(u) \leq C \mathbf{h}\left(T, \mathbf{C}_{4 s}(x), \pi_{0}\right)+C s\|D \Psi\|_{0}$.

(iii) $\mathbf{G}_{u}\left\llcorner\left(K \times \mathbb{R}^{n}\right)=T\left\llcorner\left(K \times \mathbb{R}^{n}\right) \bmod (p)\right.\right.$;

(iv) for $r_{0}:=16 \sqrt[m]{E / \delta}<1$ we have

$$
\left|B_{r}(x) \backslash K\right| \leq \frac{5^{m}}{\delta} \mathbf{e}_{T}\left(\left\{\mathbf{m e}_{T} \geq \delta\right\} \cap B_{r+r_{0} s}(x)\right) \quad \forall r \leq 3 s .
$$

We remark that in Proposition 9.6 we are not assuming that $T$ is area minimizing modulo $p$. The proof of the proposition will require a suitable $B V$ estimate for 0 -dimensional slices $\bmod (p)$, which is the content of the next section. This JerrardSoner type estimate is in fact a delicate point of the present paper, since the approach of [DLS14] (which relies on testing the current with a suitable class of differential $m$-forms) is unavailable in our setting, since Assumption 9.1 only guarantees $\partial T\left\llcorner\mathbf{C}_{4 s}(x)=0 \bmod (p)\right.$ and not $\partial T\left\llcorner\mathbf{C}_{4 s}(x)=0\right.$.

\section{A BV estimate for slices modulo $p$}

Recall that $\mathscr{F}_{k}(C)$ denotes the group of $k$-dimensional integral flat chains supported in a closed set $C$.

DeFinition 10.1. We define the groups

$$
\begin{aligned}
X & :=\left\{Z \in \mathscr{F}_{0}\left(\mathbb{R}^{n}\right): Z=\partial S \text { for some } S \in \mathscr{R}_{1}\left(\mathbb{R}^{n}\right)\right\} \\
\tilde{X}^{p} & :=\left\{Z \in \mathscr{F}_{0}\left(\mathbb{R}^{n}\right): Z=\partial S+p P \text { for some } S \in \mathscr{R}_{1}\left(\mathbb{R}^{n}\right), P \in \mathscr{F}_{0}\left(\mathbb{R}^{n}\right)\right\} .
\end{aligned}
$$

On $X$ we define the distance function

$$
\mathbf{d}_{\mathcal{F}}\left(T_{1}, T_{2}\right)=\mathcal{F}\left(T_{1}-T_{2}\right):=\inf \left\{\mathbf{M}(S): S \in \mathscr{R}_{1}\left(\mathbb{R}^{n}\right) \text { such that } T_{1}-T_{2}=\partial S\right\},
$$

whereas on $\tilde{X}^{p}$ we set

$$
\begin{array}{r}
\mathbf{d}_{\mathcal{F}^{p}}\left(T_{1}, T_{2}\right)=\mathcal{F}^{p}\left(T_{1}-T_{2}\right):=\inf \left\{\mathbf{M}(S): S \in \mathscr{R}_{1}\left(\mathbb{R}^{n}\right) \text { such that } T_{1}-T_{2}=\partial S+p P\right. \\
\text { for some } \left.P \in \mathscr{F}_{0}\left(\mathbb{R}^{n}\right)\right\} .
\end{array}
$$

REMARK 10.2. Note that the following properties are satisfied:

(i) both $X$ and $\tilde{X}^{p}$ are subgroups of $\mathscr{F}_{0}\left(\mathbb{R}^{n}\right)$, with $X \subset \tilde{X}^{p}$; 
(ii) $\tilde{X}^{p}=\left\{T \in \mathscr{F}_{0}\left(\mathbb{R}^{n}\right): T=S \bmod (p)\right.$ for some $\left.S \in X\right\}$, the non-trivial inclusion being a consequence of [MS18, Corollary 4.7]. Hence, the quotient groups $X / \bmod (p)$ and $\tilde{X}^{p} / \bmod (p)$ coincide and they are characterized by $X / \bmod (p)=$ $\tilde{X}^{p} / \bmod (p)=X^{p}$, where

$$
X^{p}:=\left\{[T] \in \mathscr{F}_{0}^{p}\left(\mathbb{R}^{n}\right): T=\partial S \bmod (p) \text { for some } S \in \mathscr{R}_{1}\left(\mathbb{R}^{n}\right)\right\} ;
$$

(iii) for $T \in X$ (resp. $\left.T \in \tilde{X}^{p}\right)$, one has $\mathcal{F}(T) \geq \mathscr{F}(T)\left(\operatorname{resp} . \mathcal{F}^{p}(T) \geq \mathscr{F}^{p}(T)\right)$;

(iv) $\left(X, \mathbf{d}_{\mathcal{F}}\right)$ is a complete metric space; the pseudo-metric $\mathbf{d}_{\mathcal{F}^{p}}$ induces a complete metric space structure on the quotient $X^{p}$, which we still denote $\mathbf{d}_{\mathcal{F}^{p}}$.

In the rest of the section we will use the theory of $B V$ maps defined over Euclidean domains and taking values in metric spaces, as established in Ambrosio's foundational paper [Amb90].

Lemma 10.3. Assume $T$ is a one-dimensional integer rectifiable current satisfying Assumption 9.1 in $\mathbf{C}_{4}$ (that is, set $m=1, x=0$ and $r=1$ in Assumption 9.1), and let $T_{t}$ be the slice $\langle T, \mathbf{p}, t\rangle \in \mathscr{R}_{0}\left(\mathbb{R}^{1+n}\right)$ for a.e. $\left.t \in B_{4}=\right]-4,4[$. Then, the map $\Phi: t \in J:=]-4,4\left[\mapsto\left[\mathbf{p}_{\sharp}^{\perp} T_{t}\right]\right.$ is in $B V\left(J, X^{p}\right)$, and moreover

$$
|D \Phi|(I)^{2} \leq 2 \mathbf{e}_{T}(I)\|T\|\left(I \times \mathbb{R}^{n}\right) \quad \text { for every Borel set } I \subset J .
$$

Proof. Let us first observe that since $(\partial T)\left\llcorner\mathbf{C}_{4}=0 \bmod (p)\right.$ then by Lemma 6.1 for a.e. $t \in J$ we have

$$
T_{t}=\partial(T\llcorner\{\mathbf{p}<t\}) \bmod (p),
$$

and thus $\Phi(t)=\left[\partial \mathbf{p}_{\sharp}^{\perp}(T\llcorner\{\mathbf{p}<t\})] \in X^{p}\right.$. Fix now $t_{0} \in J$ such that (10.2) holds. Again by Lemma 6.1 , for a.e. $t \in] t_{0}, 4\left[\right.$ we have $\Phi(t)-\Phi\left(t_{0}\right)=\left[\partial \mathbf{p}_{\sharp}^{\perp}\left(T\left\llcorner\left(\left(t_{0}, t\right) \times \mathbb{R}^{n}\right)\right)\right]\right.$. So

$$
\mathcal{F}^{p}\left(\Phi(t)-\Phi\left(t_{0}\right)\right) \leq \mathbf{M}\left(\mathbf{p}_{\sharp}^{\perp}\left(T\left\llcorner\left(\left(t_{0}, t\right) \times \mathbb{R}^{n}\right)\right)\right) .\right.
$$

Arguing analogously for the $t \in\left(-4, t_{0}\right)$ and integrating allows to conclude

$$
\int_{-4}^{4} \mathbf{d}_{\mathcal{F}^{p}}\left(\Phi(t), \Phi\left(t_{0}\right)\right) d t \leq C \mathbf{M}\left(T\left\llcorner\mathbf{C}_{4}\right),\right.
$$

which shows that $\Phi \in L^{1}\left(J, X^{p}\right)$.

Next, we pass to the proof of (10.1). Without loss of generality, assume $I=(a, b)$ to be an interval with $a$ and $b$ Lebesgue points for $\Phi$. It is a consequence of [Fed69, Theorem 4.5.9] (see also [DPH12, Section 8.1]) that $|D \Phi|(I)$ equals the classical essential variation ess $\operatorname{var}(\Phi)$ given by

$$
\text { ess } \operatorname{var}(\Phi):=\sup \left\{\sum_{i=1}^{N} \mathbf{d}_{\mathcal{F}^{p}}\left(\Phi\left(t_{i}\right), \Phi\left(t_{i-1}\right)\right): a \leq t_{0}<t_{1}<\ldots t_{N} \leq b\right.
$$

with $t_{0}, \ldots, t_{N}$ Lebesgue points for $\left.\Phi\right\}$. 
Let $t_{0}, \ldots, t_{N}$ be as in (10.5), and let $e$ denote the constant unit 1-vector orienting $\mathbb{R} \times\{0\} \subset \mathbb{R}^{1+n}$. Then, one has

$$
\begin{aligned}
\sum_{i=1}^{N} d_{\mathcal{F}^{p}}\left(\Phi\left(t_{i}\right), \Phi\left(t_{i-1}\right)\right) & =\sum_{i=1}^{N} \mathcal{F}^{p}\left(\mathbf{p}_{\sharp}^{\perp} T_{t_{i}}-\mathbf{p}_{\sharp}^{\perp} T_{t_{i-1}}\right) \leq \sum_{i=1}^{N} \mathbf{M}\left(\mathbf{p}_{\sharp}^{\perp}\left(T\left\llcorner\left(\left(t_{i-1}, t_{i}\right) \times \mathbb{R}^{n}\right)\right)\right)\right. \\
& \leq \int_{I \times \mathbb{R}^{n}}|\vec{T}-\langle\vec{T}, e\rangle e| d\|T\|=\int_{I \times \mathbb{R}^{n}} \sqrt{1-\langle\vec{T}, e\rangle^{2}} d\|T\| \\
& \leq \sqrt{2} \int_{I \times \mathbb{R}^{n}} \sqrt{1-\langle\vec{T}, e\rangle} d\|T\| \\
& \leq \sqrt{2}\left(\|T\|\left(I \times \mathbb{R}^{n}\right)-\left\|\mathbf{p}_{\sharp} T\right\|\left(I \times \mathbb{R}^{n}\right)\right)^{\frac{1}{2}}\left(\|T\|\left(I \times \mathbb{R}^{n}\right)\right)^{\frac{1}{2}} \\
& \leq \sqrt{2}\left(\mathbf{e}_{T}(I)\right)^{\frac{1}{2}}\left(\|T\|\left(I \times \mathbb{R}^{n}\right)\right)^{\frac{1}{2}},
\end{aligned}
$$

where the first inequality has been deduced analogously to (10.3), and the last one follows from $\left\|\mathbf{p}_{\sharp} T\right\|_{p} \leq\left\|\mathbf{p}_{\sharp} T\right\|$ as measures. This shows (10.1) and concludes the proof.

\section{Comparison between distances}

Another delicate point in the proof of Proposition 9.6 is that Lemma 10.3 is not powerful enough to guarantee the Lipschitz continuity of the approximating map $u$. To that aim, we shall need to combine the Jerrard-Soner type estimate (10.1) with the result of Proposition 11.1 below.

Let $Q$ and $p$ be positive integers with $Q \leq \frac{p}{2}$, and fix any $A, B \in \mathcal{A}_{Q}\left(\mathbb{R}^{n}\right)$. Observe that $A, B \in \mathscr{F}_{0}\left(\mathbb{R}^{n}\right)$. Furthermore, the flat chain $A-B$ is an element of the subgroup $X$ of Definition 10.1 , so that we can compute $\mathcal{F}(A-B)$. Next, let us consider the flat chain $A+B$. In the case when $Q=\frac{p}{2}$, we claim that $A+B \in \tilde{X}^{p}$, so that we can compute $\mathcal{F}^{p}(A+B)$. Indeed, fix any $z \in \mathbb{R}^{n}$, and let $h_{z}:(0,1) \times \mathbb{R}^{n} \rightarrow \mathbb{R}^{n}$ be the function defined by

$$
h_{z}(t, x):=z+t(x-z) .
$$

Then, the cone over $A+B$ with vertex $z$, that is the 1-dimensional integral current $R$ given by

$$
R:=z \mathbb{x}(A+B):=\left(h_{z}\right)_{\sharp}(\llbracket(0,1) \rrbracket \times(A+B))
$$

satisfies

$$
\partial R=A+B-2 Q \llbracket z \rrbracket=A+B-p \llbracket z \rrbracket,
$$

which proves our claim. Furthermore, the above argument also shows that

$$
\mathcal{F}^{p}(A+B) \leq \mathbf{M}(R)=\mathcal{F}(A-Q \llbracket z \rrbracket)+\mathcal{F}(B-Q \llbracket z \rrbracket) .
$$

Having this in mind, we extend the norm $\mathcal{F}$ to $A+B$ by setting

$$
\mathcal{F}(A+B):=\inf _{z \in \mathbb{R}^{n}}\{\mathcal{F}(A-Q \llbracket z \rrbracket)+\mathcal{F}(B-Q \llbracket z \rrbracket)\} \quad \text { when } Q=\frac{p}{2},
$$


so that (11.1) implies that

$$
\mathcal{F}^{p}(A+B) \leq \mathcal{F}(A+B) \quad \text { for every } A, B \in \mathcal{A}_{Q}\left(\mathbb{R}^{n}\right) \text { when } Q=\frac{p}{2} .
$$

The following result holds.

Proposition 11.1. Let $p$ and $Q$ be positive integers with $Q \leq \frac{p}{2}$. Let $A:=\sum_{i=1}^{Q} \llbracket A_{i} \rrbracket$ and $B:=\sum_{i=1}^{Q} \llbracket B_{i} \rrbracket$ in $\mathcal{A}_{Q}\left(\mathbb{R}^{n}\right)$, and let $\sigma \in\{-1,1\}$. If

(a) either $\sigma=1$,

(b) or $\sigma=-1$ and $Q=\frac{p}{2}$,

then

$$
\mathcal{F}^{p}(A-\sigma B)=\mathcal{F}(A-\sigma B) .
$$

The proof of Proposition 11.1 hinges upon a simple combinatorial argument. However, in order not to divert attention away from the proof of Proposition 9.6, we postpone it to "Appendix A".

\section{Proof of Proposition 9.6}

Since the statement is scaling and translation invariant, there is no loss of generality in assuming $x=0$ and $s=1$. Consider the slices $T_{x}:=\langle T, \mathbf{p}, x\rangle \in \mathscr{R}_{0}\left(\mathbb{R}^{m+n}\right)$ for a.e. $x \in \mathbb{R}^{m} \times\{0\}$ and use [Fed69, Theorem 4.3.2(2)] and [AFP00, Corollary 2.23] to conclude that

$$
\mathbf{M}\left(T_{x}\right) \leq \lim _{r \rightarrow 0} \frac{\|T\|\left(\mathbf{C}_{r}(x)\right)}{\omega_{m} r^{m}} \leq \mathbf{m e}_{T}(x)+Q \quad \text { for a.e. } x .
$$

Now, since $\mathbf{m e}_{T}(x) \leq \delta<1$ for every $x \in K$, we conclude that $\mathbf{M}\left(T_{x}\right)<Q+1$ for a.e. $x \in K$. On the other hand, setting $\mathbf{M}(x):=\mathbf{M}\left(T_{x}\right)$ for $x \in B_{4}$ we have the simple inequality

$$
\mathbf{M} \mathscr{L}^{m}\left\llcorner B_{4} \geq\left\|\mathbf{p}_{\sharp} T\right\| \geq\left\|\mathbf{p}_{\sharp} T\right\|_{p}=Q \mathscr{L}^{m}\left\llcorner B_{4},\right.\right.
$$

so that we deduce

$$
\mathbf{M}\left(T_{x}\right)=\mathbf{M}(x) \geq Q \quad \text { for a.e. } x \in B_{4} .
$$

From (12.1) and (12.3) we infer then that $\mathbf{M}\left(T_{x}\right)=Q$ for a.e. $x \in K$. Hence, there are $Q$ functions $g_{i}: K \rightarrow \mathbb{R}^{n}$ such that $\mathbf{p}_{\sharp}^{\perp} T_{x}=\sum_{i=1}^{Q} \sigma_{i}(x) \llbracket g_{i}(x) \rrbracket$ for a.e. $x \in K$, with $\sigma_{i}(x) \in\{-1,1\}$. In fact, since $\left\|\mathbf{p}_{\sharp} T\right\| \geq Q \mathscr{L}^{m}\left\llcorner B_{4}\right.$, the values of $\sigma_{i}(x)$, for fixed $x$, are independent of $i$, and thus $\mathbf{p}_{\sharp}^{\perp} T_{x}=\sigma(x) \sum_{i=1}^{Q} \llbracket g_{i}(x) \rrbracket$. Furthermore, since $\mathbf{p}_{\sharp} T=Q \llbracket B_{4} \rrbracket \bmod (p)$, it has to be $\sigma(x) Q \vec{Q} \equiv Q \bmod (p)$ as integers. We therefore have to distinguish between two cases: 
(A) $Q<\frac{p}{2}$. In this case, the condition $\sigma(x) Q \equiv Q \bmod (p)$ is satisfied if and only if $\sigma(x)=1$. Hence, the functions $g_{i}$ allow to define a measurable map $g: K \rightarrow \mathcal{A}_{Q}\left(\mathbb{R}^{n}\right)$ by setting

$$
g(x):=\sum_{i=1}^{Q} \llbracket g_{i}(x) \rrbracket .
$$

(B) $Q=\frac{p}{2}$. In this case, any measurable choice of $\sigma: K \rightarrow\{-1,1\}$ would satisfy the condition $\sigma(x) Q \equiv Q \bmod (p)$. On the other hand

$$
g(x):=\left(\sum_{i=1}^{Q} \llbracket g_{i}(x) \rrbracket, \sigma(x)\right)
$$

defines a measurable function $g: K \rightarrow \mathscr{A}_{Q}\left(\mathbb{R}^{n}\right)$.

12.1 Lipschitz estimate. Fix $j \in\{1, \ldots, m\}$, and let $\hat{\mathbf{p}}_{j}: \mathbb{R}^{m+n} \rightarrow \mathbb{R}^{m-1}$ be the orthogonal projection onto the $(m-1)$-plane given by $\operatorname{span}\left(e_{1}, \ldots, e_{j-1}, e_{j+1}, \ldots, e_{m}\right)$. For almost every $z \in \mathbb{R}^{m-1}$, consider the one-dimensional slice $T_{z}^{j}:=\left\langle T, \hat{\mathbf{p}}_{j}, z\right\rangle$, and observe that

$$
\int_{\mathbb{R}^{m-1}} \mathbf{M}\left(T_{z}^{j}\right) d z \leq \mathbf{M}(T)
$$

Observe that $T_{z}^{j}$ satisfies Assumption 9.1 with $m=1$ for a.e. $z$. Let now $\mathbf{p}_{j}$ be the orthogonal projection $\mathbf{p}_{j}: \mathbb{R}^{m+n} \rightarrow \operatorname{span}\left(e_{j}\right)$, and for almost every $t \in \mathbb{R}$ let $\left(T_{z}^{j}\right)_{t}:=\left\langle T_{z}^{j}, \mathbf{p}_{j}, t\right\rangle$. By Lemma 10.3, the map $\Phi_{z}^{j}: t \mapsto \mathbf{p}_{\sharp}^{\perp}\left(T_{z}^{j}\right)_{t}$ is $B V\left(\mathbb{R}, X^{p}\right)$, and moreover

$$
\left|D \Phi_{z}^{j}\right|(I)^{2} \leq 2 \mathbf{e}_{T_{z}^{j}}(I)\left\|T_{z}^{j}\right\|\left(I \times \mathbb{R}^{n}\right) \quad \text { for every Borel set } I \subset B_{4} \cap \operatorname{span}\left(e_{j}\right) .
$$

Now, observe that

$$
\begin{aligned}
\Phi_{z}^{j}(t) & =\mathbf{p}_{\sharp}^{\perp}\left(T_{z}^{j}\right)_{t}=\mathbf{p}_{\sharp}^{\perp}\left\langle\left\langle T, \hat{\mathbf{p}}_{j}, z\right\rangle, \mathbf{p}_{j}, t\right\rangle=(-1)^{m-j} \mathbf{p}_{\sharp}^{\perp}\langle T, \mathbf{p}, x(j, z, t)\rangle \\
& =(-1)^{m-j} \mathbf{p}_{\sharp}^{\perp} T_{x(j, z, t)},
\end{aligned}
$$

where $x(j, z, t):=\left(z_{1}, \ldots, z_{j-1}, t, z_{j+1}, \ldots, z_{m}\right) \in \mathbb{R}^{m}$. By [DPH12, formula (79)], we can therefore conclude that the map $\Phi: x \in \mathbb{R}^{m} \mapsto \mathbf{p}_{\sharp}^{\perp} T_{x}$ is in $B V\left(\mathbb{R}^{m}, X^{p}\right)$. Furthermore, if for every Borel set $A \subset B_{4}$, for every $j \in\{1, \ldots, m\}$ and for every $z=\left(z_{1}, \ldots, z_{j-1}, z_{j+1}, \ldots, z_{m}\right) \in \mathbb{R}^{m-1}$ we denote 
$A_{z}^{j}:=\left\{t \in \mathbb{R}:\left(z_{1}, \ldots, z_{j-1}, t, z_{j+1}, \ldots, z_{m}\right) \in A\right\}$, we have

$$
\begin{aligned}
|D \Phi|(A) & \leq \sum_{j=1}^{m} \int_{\mathbb{R}^{m-1}}\left|D \Phi_{z}^{j}\right|\left(A_{z}^{j}\right) d z \\
& \stackrel{(10.1)}{\leq} \sqrt{2} \sum_{j=1}^{m} \int_{\mathbb{R}^{m-1}}\left(\mathbf{e}_{T_{z}^{j}}\left(A_{z}^{j}\right)\right)^{\frac{1}{2}}\left(\left\|T_{z}^{j}\right\|\left(A_{z}^{j} \times \mathbb{R}^{n}\right)\right)^{\frac{1}{2}} d z \\
& \leq \sqrt{2} \sum_{j=1}^{m}\left(\int_{\mathbb{R}^{m-1}} \mathbf{e}_{T_{z}^{j}}\left(A_{z}^{j}\right) d z\right)^{\frac{1}{2}}\left(\int_{\mathbb{R}^{m-1}}\left\|T_{z}^{j}\right\|\left(A_{z}^{j} \times \mathbb{R}^{n}\right) d z\right)^{\frac{1}{2}} \\
& \leq \sqrt{2} m\left(\mathbf{e}_{T}(A)\right)^{\frac{1}{2}}\left(\|T\|\left(A \times \mathbb{R}^{n}\right)\right)^{\frac{1}{2}} .
\end{aligned}
$$

Thus, from the definition of excess measure modulo $p$ we deduce

$$
|D \Phi|\left(B_{r}(y)\right)^{2} \leq 2 m^{2} \mathbf{e}_{T}\left(B_{r}(y)\right)\left(Q\left|B_{r}(y)\right|+\mathbf{e}_{T}\left(B_{r}(y)\right)\right),
$$

for any $B_{r}(y) \subset B_{4}$. Hence, if we define the maximal function

$$
\mathbf{m}|D \Phi|(x):=\sup _{x \in B_{r}(y) \subset B_{4}} \frac{|D \Phi|\left(B_{r}(y)\right)}{\left|B_{r}(y)\right|},
$$

we can conclude that

$$
(\mathbf{m}|D \Phi|(x))^{2} \leq 2 m^{2}\left(Q \mathbf{m e}_{T}(x)+\left(\mathbf{m e}_{T}(x)\right)^{2}\right) \leq C \delta \quad \text { for every } x \in K .
$$

By [AK00, Lemma 7.3], one immediately obtains

$$
\mathcal{F}^{p}(\Phi(x)-\Phi(y)) \leq C \delta^{1 / 2}|x-y| \quad \text { for every } x, y \in K \text { Lebesgue point of } \Phi .
$$

On the other hand, for a.e. $x \in K$ we can regard $\Phi(x)=g(x) \in \mathcal{A}_{Q}\left(\mathbb{R}^{n}\right)$ if $Q<\frac{p}{2}$ or $\Phi(x)=\sigma(x) g_{0}(x)$ with $\sigma(x) \in\{-1,1\}$ and $g_{0}(x) \in \mathcal{A}_{Q}\left(\mathbb{R}^{n}\right)$ if $Q=\frac{p}{2}$. In any case, Proposition 11.1 implies that in fact

$$
\mathcal{F}(\Phi(x)-\Phi(y)) \leq C \delta^{1 / 2}|x-y| \quad \text { for every } x, y \in K \text { Lebesgue point of } \Phi .
$$

Now, first consider the case $Q<\frac{p}{2}$. Writing $\Phi(\cdot)=g(\cdot)$, we observe that

$$
\begin{aligned}
\mathcal{F}(g(x)-g(y)) & =\min _{\sigma \in \mathcal{P}_{Q}} \sum_{i=1}^{Q}\left|g_{i}(x)-g_{\sigma(i)}(y)\right| \geq \min _{\sigma \in \mathcal{P}_{Q}}\left(\sum_{i=1}^{Q}\left|g_{i}(x)-g_{\sigma(i)}(y)\right|^{2}\right)^{1 / 2} \\
& =\mathcal{G}(g(x), g(y)),
\end{aligned}
$$

where $\mathcal{P}_{Q}$ denotes the group of permutations of $\{1, \ldots, Q\}$.

If $Q=\frac{p}{2}$, instead, we have $\Phi(\cdot)=\sigma(\cdot) g_{0}(\cdot)$. If $\sigma(x)=\sigma(y)$, then the same computation produces

$$
\mathcal{F}\left(\sigma(x) g_{0}(x)-\sigma(y) g_{0}(y)\right) \geq \mathcal{G}\left(g_{0}(x), g_{0}(y)\right)=\mathcal{G}_{s}(g(x), g(y)) .
$$


If, on the other hand, $\sigma(x) \neq \sigma(y)$, and to fix the ideas say that $\sigma(x)=1$ and $\sigma(y)=-1$, then

$$
\begin{aligned}
\mathcal{F}\left(g_{0}(x)+g_{0}(y)\right): & =\inf _{z \in \mathbb{R}^{n}}\left\{\mathcal{F}\left(g_{0}(x)-Q \llbracket z \rrbracket\right)+\mathcal{F}\left(g_{0}(y)-Q \rrbracket z \rrbracket\right)\right\} \\
& \geq \inf _{z \in \mathbb{R}^{n}}\left\{\mathcal{G}\left(g_{0}(x), Q \llbracket z \rrbracket\right)+\mathcal{G}\left(g_{0}(y), Q \rrbracket z \rrbracket\right)\right\} \\
& \geq \inf _{z \in \mathbb{R}^{n}}\left(\mathcal{G}\left(g_{0}(x), Q \llbracket z \rrbracket\right)^{2}+\mathcal{G}\left(g_{0}(y), Q \llbracket z \rrbracket\right)^{2}\right)^{1 / 2} .
\end{aligned}
$$

Now observe that

$$
\begin{aligned}
& \mathcal{G}\left(g_{0}(x), Q \llbracket z \rrbracket\right)^{2}+\mathcal{G}\left(g_{0}(y), Q \llbracket z \rrbracket\right)^{2} \\
& \quad=\left|g_{0}(x) \ominus \boldsymbol{\eta} \circ g_{0}(x)\right|^{2}+\left|g_{0}(y) \ominus \boldsymbol{\eta} \circ g_{0}(y)\right|^{2}+Q\left|\boldsymbol{\eta} \circ g_{0}(x)-z\right|^{2}+Q\left|\boldsymbol{\eta} \circ g_{0}(y)-z\right|^{2} .
\end{aligned}
$$

Thus

$$
\begin{aligned}
& \inf _{z \in \mathbb{R}^{n}}\left(\mathcal{G}\left(g_{0}(x), Q \llbracket z \rrbracket\right)^{2}+\mathcal{G}\left(g_{0}(y), Q \llbracket z \rrbracket\right)^{2}\right) \\
& \quad=\left|g_{0}(x) \ominus \boldsymbol{\eta} \circ g_{0}(x)\right|^{2}+\left|g_{0}(y) \ominus \boldsymbol{\eta} \circ g_{0}(y)\right|^{2}+\frac{Q}{2}\left|\boldsymbol{\eta} \circ g_{0}(x)-\boldsymbol{\eta} \circ g_{0}(y)\right|^{2} . \\
& \quad \geq \frac{1}{2} \mathcal{G}_{s}\left(g_{0}(x), g_{0}(y)\right)^{2} .
\end{aligned}
$$

This shows that $g \in \operatorname{Lip}\left(K, \mathcal{A}_{Q}\left(\mathbb{R}^{n}\right)\right)$ (resp. $g \in \operatorname{Lip}\left(K, \mathscr{A}_{Q}\left(\mathbb{R}^{n}\right)\right)$ with $\operatorname{Lip}(g) \leq$ $C \delta^{1 / 2}$.

12.2 Conclusion. Next, in case $Q<\frac{p}{2}$, write

$$
g(x)=\sum_{i} \llbracket\left(h_{i}(x), \Psi\left(x, h_{i}(x)\right)\right) \rrbracket .
$$

Obviously, $x \mapsto h(x):=\sum_{i} \llbracket h_{i}(x) \rrbracket \in \mathcal{A}_{Q}\left(\mathbb{R}^{\bar{n}}\right)$ is a Lipschitz map on $K$ with Lipschitz constant $\leq C \delta^{1 / 2}$. Recalling [DLS11, Theorem 1.7], we can extend it to a map $\bar{h} \in$ $\operatorname{Lip}\left(B_{3}, \mathcal{A}_{Q}\left(\mathbb{R}^{\bar{n}}\right)\right)$ satisfying $\operatorname{Lip}(\bar{h}) \leq C \delta^{1 / 2}$ (for a possibly larger $C$ ) and osc $(\bar{h}) \leq$ Cosc $(h)$. Finally, set

$$
u(x):=\sum_{i} \llbracket\left(\bar{h}_{i}(x), \Psi\left(x, \bar{h}_{i}(x)\right)\right) \rrbracket .
$$

The same computations of [DLS14, Section 3.2] then show the Lipschitz and the oscillation bound in Claim (ii) of the Proposition.

For $Q=\frac{p}{2}$ we argue analogously, using this time the Extension Corollary [DLHMS, Corollary 5.3] in place of [DLS11, Theorem 1.7].

Note that the points (i) and (iii) of the proposition are obvious by construction. Next observe that, since $\mathbf{m e}_{T}$ is lower semicontinuous, $K$ is obviously closed. Let $U:=\left\{\mathbf{m e}_{T}>\delta\right\}$ be its complement. Fix $r \leq 3$ and for every point $x \in U \cap B_{r}$ 
consider a ball $B^{x}$ of radius $r(x)$ which contains $x$ and satisfies $\mathbf{e}_{T}\left(B^{x}\right)>\delta \omega_{m} r(x)^{m}$. Since $\mathbf{e}_{T}\left(B^{x}\right) \leq E$ we obviously have

$$
r(x)<\sqrt[m]{\frac{E}{\omega_{m} \delta}}<r_{0}<1
$$

Now, by the definition of the maximal function it follows clearly that $B^{x} \subset U \cap B_{r+r_{0}}$. In turn, by the $5 r$ covering theorem we can select countably many pairwise disjoint $B^{x_{i}}$ such that the corresponding concentric balls $\hat{B}^{i}$ with radii $5 r\left(x_{i}\right)$ cover $U \cap B_{r}$. Then we get

$$
\left|U \cap B_{r}\right| \leq 5^{m} \sum_{i} \omega_{m} r\left(x_{i}\right)^{m} \leq \frac{5^{m}}{\delta} \sum_{i} \mathbf{e}_{T}\left(B^{x_{i}}\right) \leq \frac{5^{m}}{\delta} \mathbf{e}_{T}\left(U \cap B_{r+r_{0}}\right) .
$$

This shows claim (iv) of the proposition and completes the proof.

\section{First harmonic approximation}

REMARK 13.1. (Good system of coordinates) Let $T$ be as in Assumption 9.1 in the cylinder $\mathbf{C}_{4 r}(x)$. If the excess $E=\mathbf{E}\left(T, \mathbf{C}_{4 r}(x)\right)$ is smaller than a geometric constant, then without loss of generality we can assume that the function $\Psi: \mathbb{R}^{m+\bar{n}} \rightarrow \mathbb{R}^{l}$ parametrizing the manifold $\Sigma$ satisfies $\Psi(0)=0,\|D \Psi\|_{0} \leq C\left(E^{1 / 2}+r \mathbf{A}\right)$ and $\left\|D^{2} \Psi\right\|_{0} \leq C \mathbf{A}$. This can be shown using a small variation of the argument outlined in [DLS14, Remark 2.5]. First of all, as anticipated in Remark 9.3, we introduce a suitable notion of nonoriented excess. Given the plane $\pi_{0}$ we consider the $m$-vector $\vec{\pi}_{0}$ of mass 1 which gives the standard orientation to it. We then let

$$
\left|\vec{T}(y)-\pi_{0}\right|_{n o}:=\min \left\{\left|\vec{T}(y)-\vec{\pi}_{0}\right|,\left|\vec{T}(y)+\vec{\pi}_{0}\right|\right\}
$$

where $|\cdot|$ is the norm associated to the standard inner product on the space $\Lambda_{m}\left(\mathbb{R}^{m+n}\right)$ of $m$-vectors in $\mathbb{R}^{m+n}$, and define

$$
\mathbf{E}^{n o}\left(T, \mathbf{C}_{4 r}(x)\right)=\frac{1}{2 \omega_{m}(4 r)^{m}} \int_{\mathbf{C}_{4 r}(x)}\left|\vec{T}(y)-\pi_{0}\right|_{n o}^{2} d\|T\|(y) .
$$

Consider next the orthogonal projection $\mathbf{p}: \mathbb{R}^{m+n} \rightarrow \pi_{0}$ and the corresponding slices $\langle T, \mathbf{p}, y\rangle$ with $y \in B_{4 r}(x)$. For a.e. $y$, such a slice is an integral 0-dimensional current and we let $\mathbf{M}(y) \in \mathbb{N}$ be its mass. Once again (cf. (12.2)), we observe that under the Assumption 9.1 we have

$$
\mathbf{M} \mathscr{L}^{m}\left\llcorner B_{4 r}(x) \geq\left\|\mathbf{p}_{\sharp} T\right\| \geq\left\|\mathbf{p}_{\sharp} T\right\|_{p}=Q \mathscr{L}^{m}\left\llcorner B_{4 r}(x) .\right.\right.
$$


Thus, an elementary computation gives

$$
\begin{aligned}
\mathbf{E}^{n o}\left(T, \mathbf{C}_{4 r}(x)\right) & =\frac{1}{\omega_{m}(4 r)^{m}}\left(\|T\|\left(\mathbf{C}_{4 r}(x)\right)-\int_{B_{4 r}(x)} \mathbf{M}(y) d y\right) \\
& \leq \frac{1}{\omega_{m}(4 r)^{m}}\left(\|T\|\left(\mathbf{C}_{4 r}(x)\right)-\left\|\mathbf{p}_{\sharp} T\right\|\left(\mathbf{C}_{4 r}(x)\right)\right) \\
& \leq \frac{1}{\omega_{m}(4 r)^{m}}\left(\|T\|\left(\mathbf{C}_{4 r}(x)\right)-\left\|\mathbf{p}_{\sharp} T\right\|_{p}\left(\mathbf{C}_{4 r}(x)\right)\right) \\
& =\mathbf{E}\left(T, \mathbf{C}_{4 r}(x)\right)=E .
\end{aligned}
$$

At this point we find clearly a point $q \in \operatorname{spt}(T) \cap \mathbf{C}_{4 r}(x)$ such that

$$
\min \left\{\left|\vec{T}(q)-\vec{\pi}_{0}\right|,\left|\vec{T}(q)-\left(-\vec{\pi}_{0}\right)\right|\right\} \leq C E^{1 / 2}
$$

and we can proceed with the very same argument of [DLS14, Remark 3.5].

Definition 13.2 ( $E^{\beta}$-Lipschitz approximation). Let $\beta \in\left(0, \frac{1}{2 m}\right)$, let $T$ be as in Proposition 9.6 such that $32 E^{\frac{1-2 \beta}{m}}<1$. If the coordinates are fixed as in Remark 13.1, then the Lipschitz approximation of $T$ provided by Proposition 9.6 corresponding to the choice $\delta=E^{2 \beta}$ will be called the $E^{\beta}$-Lipschitz approximation of $T$ in $\mathbf{C}_{3 s}(x)$.

In the following theorem, we show that the minimality assumption on the current $T$ and the smallness of the excess imply that the $E^{\beta}$-Lipschitz approximation of $T$ in $\mathbf{C}_{3 s}(x)$ is close to a Dirichlet minimizer $h$, and we quantify the distance between $u$ and $h$ in terms of the excess.

Theorem 13.3. For every $\eta_{*}>0$ and every $\beta \in\left(0, \frac{1}{2 m}\right)$ there exist constants $\varepsilon_{*}>0$ and $C>0$ with the following property. Let $T$ and $\Psi$ be as in Assumption 9.1 in the cylinder $\mathbf{C}_{4 s}(x)$, and assume that $T$ is area $\operatorname{minimizing} \bmod (p)$ in there. Let $u$ be the $E^{\beta}$-Lipschitz approximation of $T$ in $B_{3 s}(x)$, and let $K$ be the set satisfying all the properties of Proposition 9.6 for $\delta=E^{2 \beta}$. If $E \leq \varepsilon_{*}$ and $s \mathbf{A} \leq \varepsilon_{*} E^{\frac{1}{2}}$, then

$$
\mathbf{e}_{T}\left(B_{5 s / 2} \backslash K\right) \leq \eta_{*} E s^{m},
$$

and

$$
\operatorname{Dir}\left(u, B_{2 s}(x) \backslash K\right) \leq C \eta_{*} E s^{m} .
$$

Moreover, there exists a map $h$ defined on $B_{3 s}(x)$ and taking either values in $\mathcal{A}_{Q}\left(\mathbb{R}^{n}\right)$, if $Q<\frac{p}{2}$, or in $\mathscr{A}_{Q}\left(\mathbb{R}^{n}\right)$, if $Q=\frac{p}{2}$, for which the following facts hold:

(i) $h(x)=(\bar{h}(x), \Psi(x, \bar{h}(x))$ with $\bar{h}$ Dirichlet minimizing;

(ii)

$$
\begin{gathered}
s^{-2} \int_{B_{2 s}(x)} \mathcal{G}_{s}(u, h)^{2}+\int_{B_{2 s}(x)}(|D u|-|D h|)^{2} \leq \eta_{*} E s^{m} \\
\int_{B_{2 s}(x)}|D(\boldsymbol{\eta} \circ u)-D(\boldsymbol{\eta} \circ h)|^{2} \leq \eta_{*} E s^{m} .
\end{gathered}
$$


REMARK 13.4. There exists a dimensional constant $c$ such that, if $E \leq c$ and $s \mathbf{A} \leq$ $E^{1 / 2}$, then the $E^{\beta}$-Lipschitz approximation $u$ of $T$ in $\mathbf{C}_{3 s}(x)$ satisfies:

$$
\begin{aligned}
& \operatorname{Lip}(u) \leq C E^{\beta}, \\
& \operatorname{Dir}\left(u, B_{3 s}(x)\right) \leq C E s^{m} .
\end{aligned}
$$

Equation (13.7) follows from property (ii) of the Lipschitz approximation in Proposition 9.6, the choice of $\delta=E^{2 \beta}$, and the scaling of $\mathbf{A}$. The estimate in (13.8), instead, is a consequence of the Taylor expansion of the mass of multiple valued graphs deduced in [DLHMS, Corollary 13.2]. Indeed, the remainder term in equation [DLHMS, Equation (13.5)] can be estimated by

$$
\int_{B_{3 s}(x)} \sum_{i} \bar{R}_{4}\left(D u_{i}\right) \leq C \int_{B_{3 s}(x)}|D u|^{4} \leq C E^{2 \beta} \operatorname{Dir}\left(u, B_{3 s}(x)\right)<\frac{1}{4} \operatorname{Dir}\left(u, B_{3 s}(x)\right)
$$

for suitably small E. Hence, [DLHMS, Equation (13.5)] yields

$$
\begin{aligned}
\frac{1}{4} \operatorname{Dir}\left(u, B_{3 s}(x)\right) & \leq\left\|\mathbf{G}_{u}\right\|\left(\mathbf{C}_{3 s}(x)\right)-Q \omega_{m}(3 s)^{m} \\
& \leq\left(\|T\|\left(\mathbf{C}_{3 s}(x)\right)-Q \omega_{m}(3 s)^{m}\right)+\left\|\mathbf{G}_{u}\right\|\left(\left(B_{3 s}(x) \backslash K\right) \times \mathbb{R}^{n}\right) \\
& \leq \omega_{m} E(3 s)^{m}+C E^{2 \beta}\left|B_{3 s}(x) \backslash K\right| \leq C E s^{m}
\end{aligned}
$$

by property (iv) in Proposition 9.6.

Proof. Let us first observe that (13.3) implies (13.4): indeed, the estimate (9.5) implies:

$$
\operatorname{Dir}\left(u, B_{2 s}(x) \backslash K\right) \leq \operatorname{Lip}(u)^{2}\left|B_{2 s}(x) \backslash K\right| \leq C \mathbf{e}_{T}\left(B_{\frac{5}{2} s}(x) \backslash K\right) .
$$

Then, note that we can embed $\mathcal{A}_{Q}\left(\mathbb{R}^{n}\right)$ naturally and isometrically into $\mathscr{A}_{Q}\left(\mathbb{R}^{n}\right)$ using the map $T \in \mathcal{A}_{Q}\left(\mathbb{R}^{n}\right) \mapsto(T, 1)$. Hence, without loss of generality we may assume that $u$ takes values in $\mathscr{A}_{Q}\left(\mathbb{R}^{n}\right)$. Furthermore, each Lipschitz approximation is of the form $u(x)=(\bar{u}(x), \Psi(x, \bar{u}))$ with $\bar{u}$ taking values in $\mathscr{A}_{Q}\left(\mathbb{R}^{\bar{n}}\right)$.

Finally, since the statement is scale invariant we may assume $x=0$ and $s=1$.

We will now show the following.

Given any sequence of currents $T_{k}$ supported in manifolds $\Sigma_{k}=\operatorname{Gr}\left(\Psi_{k}\right)$ and corresponding Lipschitz approximations $u_{k}$ satisfying all the assumptions in $B_{3}$ with

$$
E_{k} \rightarrow 0 \quad \text { and } \quad \mathbf{A}_{k}=o\left(E_{k}^{\frac{1}{2}}\right) \quad \text { as } k \rightarrow \infty,
$$

then the following conclusions hold:

(i)

$$
\mathbf{e}_{T_{k}}\left(B_{\frac{5}{2}} \backslash K_{k}\right)=o\left(E_{k}\right)
$$


(ii) One of the following holds true: either there is a single Dirichlet minimzing map $\bar{h} \in W^{1,2}\left(B_{\frac{5}{2}}, \mathscr{A}_{Q}\left(\mathbb{R}^{\bar{n}}\right)\right)$ such that

$$
\int_{B_{s}} \mathcal{G}_{s}\left(E_{k}^{-\frac{1}{2}} \bar{u}_{k}, \bar{h}\right)^{2}+\left(E_{k}^{-\frac{1}{2}}\left|D \bar{u}_{k}\right|-|D \bar{h}|\right)^{2}=o(1) \quad \text { for all } s<\frac{5}{2} ;
$$

or there are Dirichlet minimizing maps $h_{j} \in W^{1,2}\left(B_{\frac{5}{2}}, \mathcal{A}_{Q_{j}}\left(\mathbb{R}^{\bar{n}}\right)\right.$ with $j=$ $1, \ldots, J, \sum_{j} Q_{j}=Q$, and sequences $\left\{y_{j, k}\right\}_{k \in \mathbb{N}} \in \mathbb{R}^{\bar{n}}$ such that if we consider the sequence of maps in $W^{1,2}\left(B_{\frac{5}{2}}, \mathscr{A}_{Q}\left(\mathbb{R}^{\bar{n}}\right)\right)$ given by

$$
\bar{h}_{k}:=\left(\sum_{j} \llbracket y_{j, k} \oplus h_{j} \rrbracket, \sigma\right)
$$

with $\sigma \in\{-1,1\}$ fixed we have

$$
\int_{B_{s}} \mathcal{G}_{s}\left(E_{k}^{-\frac{1}{2}} \bar{u}_{k}, \bar{h}_{k}\right)^{2}+\left(E_{k}^{-\frac{1}{2}}\left|D \bar{u}_{k}\right|-\left|D \bar{h}_{k}\right|\right)^{2}=o(1) \quad \text { for all } s<\frac{5}{2} .
$$

For sufficiently large $k$ the conclusion of the Theorem therefore holds, since we can replace in point (ii) $\bar{u}_{k}$ by $u_{k}$ and $\bar{h}_{k}$ by $h_{k}=\left(\bar{h}_{k}, E_{k}^{-\frac{1}{2}} \Psi_{k}\left(\cdot, E_{k}^{\frac{1}{2}} \bar{h}_{k}\right)\right)$. This can be seen as follows. Recall that by remark 13.1, we have $\left\|D \Psi_{k}\right\|_{0}+\left\|D^{2} \Psi_{k}\right\|_{0}=O\left(E_{k}^{\frac{1}{2}}\right)$. As a first step, we may replace in (ii) $\left(E_{k}^{-\frac{1}{2}}\left|D \bar{u}_{k}\right|-|D \bar{h}|\right)^{2}$ by $\left.\left|E_{k}^{-1}\right| D \bar{u}_{k}\right|^{2}-|D \bar{h}|^{2} \mid$. Indeed, for any sequence of non-negative measurable functions $a_{k}, b_{k}$ we have

$$
\begin{aligned}
\int\left|a_{k}-b_{k}\right|^{2} & \leq \int\left|a_{k}^{2}-b_{k}^{2}\right|=\int\left|a_{k}+b_{k}\right|\left|a_{k}-b_{k}\right| \\
& \leq 2\left(\int\left|b_{k}\right|^{2}\right)^{\frac{1}{2}}\left(\int\left|a_{k}-b_{k}\right|^{2}\right)^{\frac{1}{2}}+\int\left|a_{k}-b_{k}\right|^{2} ;
\end{aligned}
$$

hence $\left\|a_{k}-b_{k}\right\|_{2}=o(1)$ if and only if $\left\|\left(a_{k}\right)^{2}-\left(b_{k}\right)^{2}\right\|_{1}=o(1)$. Thus it remains to show that $\left.E_{k}^{-1} \int_{B_{s}}|| D \Psi_{k}\left(\cdot, \bar{u}_{k}\right)\right|^{2}-\left|D \Psi_{k}\left(\cdot, E_{k}^{\frac{1}{2}} \bar{h}_{k}\right)\right|^{2} \mid$ is $o(1)$. We compute explicitly:

$$
\begin{aligned}
\sum_{i=1}^{Q} & \left.E_{k}^{-1} \int_{B_{s}}|| D \Psi_{k}\left(\cdot, \bar{u}_{k}^{i}\right)\right|^{2}-\left|D \Psi_{k}\left(\cdot, E_{k}^{\frac{1}{2}} \bar{h}_{k}^{i}\right)\right|^{2} \mid \\
= & \sum_{i=1}^{Q} E_{k}^{-1} \int_{B_{s}}|| D_{x} \Psi_{k}\left(\cdot, \bar{u}_{k}^{i}\right)+\left.D_{y} \Psi\left(x, \bar{u}_{k}^{i}\right) D \bar{u}_{k}^{i}\right|^{2} \\
& -\left|D_{x} \Psi_{k}\left(\cdot, E_{k}^{\frac{1}{2}} \bar{h}_{k}^{i}\right)+D_{y} \Psi\left(x, E_{k}^{\frac{1}{2}} \bar{h}_{k}^{i}\right) E^{\frac{1}{2}} D \bar{h}_{k}^{i}\right|^{2} \mid \\
\leq & \left.\sum_{i=1}^{Q} \int_{B_{s}} E_{k}^{-1}|| D_{x} \Psi_{k}\left(\cdot, \bar{u}_{k}^{i}\right)\right)\left.\right|^{2}-\left|D_{x} \Psi_{k}\left(\cdot, E_{k}^{\frac{1}{2}} \bar{h}_{k}^{i}\right)\right|^{2} \mid \\
& +E_{k}^{-\frac{1}{2}} C_{k}^{1}(x)\left(E_{k}^{-\frac{1}{2}}\left|D \bar{u}_{k}^{i}\right|+\int_{B_{s}} E_{k}^{-1}\left|D \bar{u}_{k}^{i}\right|^{2}\right)+E_{k}^{-\frac{1}{2}} C_{k}^{2}(x)\left(\left|D \bar{h}_{k}^{i}\right|+\int_{B_{s}} E_{k}^{\frac{1}{2}}\left|D \bar{h}_{k}^{i}\right|^{2}\right),
\end{aligned}
$$


where the measurable functions $C_{k}^{j}(x), j=1,2$, consist of a product of two first derivatives of $\Psi_{k}$, and hence $\left.\left\|C_{k}^{j}\right\|_{0}=O\left(E_{k}\right)\right)$. Since $E_{k}^{-1} \operatorname{Dir}\left(\bar{u}_{k}, B_{\frac{5}{2}}\right), \operatorname{Dir}\left(\bar{h}_{k}, B_{\frac{5}{2}}\right)$ are uniformly bounded by (13.8), the last two integrals are $o(1)$.

The remaining term can be estimated by

$$
\begin{aligned}
\int_{B_{s}} & \left.\sum_{i=1}^{Q} E_{k}^{-1}|| D_{x} \Psi_{k}\left(\cdot, \bar{u}_{k}^{i}\right)\right)\left.\right|^{2}-\left|D_{x} \Psi_{k}\left(\cdot, E_{k}^{\frac{1}{2}} \bar{h}_{k}^{i}\right)\right|^{2} \mid \\
\leq & \int_{B_{s}} \sum_{i=1}^{Q} E_{k}^{-\frac{1}{2}}\left|D_{x} \Psi_{k}\left(\cdot, \bar{u}_{k}^{i}\right)+D_{x} \Psi_{k}\left(\cdot, E_{k}^{\frac{1}{2}} \bar{h}_{k}^{i}\right)\right| E_{k}^{-\frac{1}{2}}\left|D_{x} \Psi_{k}\left(\cdot, \bar{u}_{k}^{i}\right)-D_{x} \Psi_{k}\left(\cdot, E_{k}^{\frac{1}{2}} \bar{h}_{k}^{i}\right)\right| \\
\leq C & \int_{B_{s}} E_{k}^{-\frac{1}{2}}\left\|D \Psi_{k}\right\|_{0}\left\|D^{2} \Psi_{k}\right\|_{0} \mathcal{G}_{s}\left(E_{k}^{-\frac{1}{2}} \bar{u}_{k}, \bar{h}_{k}\right)=o(1) .
\end{aligned}
$$

13.1 Construction of the maps $\overline{\boldsymbol{h}}$ or $\boldsymbol{h}_{\boldsymbol{j}}$. Let $\iota$ be the isometry defined in [DLHMS, Proposition 2.6], and define $\left(\bar{v}_{k}, \bar{w}_{k}, \boldsymbol{\eta} \circ \bar{u}_{k}\right)=\iota \circ \bar{u}_{k}$. As in [DLHMS, Definition 2.7], we set

$$
\begin{aligned}
& B_{+}^{k}:=\left\{x \in B_{\frac{5}{2}}:\left|\bar{v}_{k}\right|=\left|\bar{u}_{k}^{+} \ominus \boldsymbol{\eta} \circ \bar{u}_{k}\right|>0\right\} \quad \text { and } \\
& B_{-}^{k}:=\left\{x \in B_{\frac{5}{2}}:\left|\bar{w}_{k}\right|=\left|\bar{u}_{k}^{-} \ominus \boldsymbol{\eta} \circ \bar{u}_{k}\right|>0\right\} .
\end{aligned}
$$

We distinguish if the limit

$$
\limsup _{k \rightarrow \infty} \min \left\{\left|B_{+}^{k}\right|,\left|B_{-}^{k}\right|\right\}=: b
$$

satisfies $b>0$ or $b=0$.

Case $b>0$ : After translating the currents $T_{k}$ vertically we may assume without loss of generality that $f_{B_{\frac{5}{2}}} \boldsymbol{\eta} \circ \bar{u}_{k}=0$ for all $k$. Since both $\bar{v}_{k}$ and $\bar{w}_{k}$ vanish on sets of measure at least $b>0$, we claim that there exists a constant $C=C_{b}$ such that

$$
\int_{B_{\frac{5}{2}}}\left|\bar{u}_{k}\right|^{2} \leq C_{b} E_{k}
$$

Indeed, observe that the classical Poincaré inequality gives

$$
\begin{aligned}
\int_{B_{\frac{5}{2}}}\left|\bar{u}_{k}\right|^{2} & =\int_{B_{\frac{5}{2}}}\left|\bar{u}_{k} \ominus \boldsymbol{\eta} \circ \bar{u}_{k}\right|^{2}+Q \int_{B_{\frac{5}{2}}}\left|\boldsymbol{\eta} \circ \bar{u}_{k}\right|^{2} \\
& =\int_{B_{\frac{5}{2}}}\left|\bar{v}_{k}\right|^{2}+\int_{B_{\frac{5}{2}}}\left|\bar{w}_{k}\right|^{2}+Q \int_{B_{\frac{5}{2}}}\left|\boldsymbol{\eta} \circ \bar{u}_{k}\right|^{2} \\
& \leq\left. C_{b} \int_{B_{\frac{5}{2}}}|D| \bar{v}_{k}\right|^{2}+\left.C_{b} \int_{B_{\frac{5}{2}}}|D| \bar{w}_{k}\right|^{2}+C_{b} \int_{B_{\frac{5}{2}}}\left|D \boldsymbol{\eta} \circ \bar{u}_{k}\right|^{2} \leq C_{b} \operatorname{Dir}\left(\bar{u}_{k}, B_{\frac{5}{2}}\right),
\end{aligned}
$$

which implies (13.9) again by (13.8).

Modulo passing to an appropriate subsequence, we therefore have that

$$
E_{k}^{-\frac{1}{2}} \bar{u}_{k} \rightarrow \bar{h}
$$


weakly in $W^{1,2}\left(B_{\frac{5}{2}}, \mathscr{A}_{Q}\left(\mathbb{R}^{\bar{n}}\right)\right)$.

Case $b=0$ : We assume that $\left|B_{-}^{k}\right| \rightarrow 0$, the other case being equivalent. Consider the map $\bar{u}_{k}^{+}$in $W^{1,2}\left(B_{\frac{5}{2}}, \mathcal{A}_{Q}\left(\mathbb{R}^{\bar{n}}\right)\right)$. When needed, we may identify $\bar{u}_{k}^{+}$with $\left(\bar{u}_{k}^{+}, 1\right)$ taking values in $\mathscr{A}_{Q}\left(\mathbb{R}^{\bar{n}}\right)$. We note that

$$
\begin{aligned}
\operatorname{Dir}\left(\bar{u}_{k}^{+}, B_{\frac{5}{2}}\right) & \leq C \operatorname{Dir}\left(\bar{u}_{k}, B_{\frac{5}{2}}\right) \leq C E_{k} ; \\
\int_{B_{\frac{5}{2}}} \mathcal{G}_{s}\left(\bar{u}_{k}, \bar{u}_{k}^{+}\right)^{2} & =\int_{B_{-}^{k}}\left|\bar{u}_{k}^{-} \ominus \boldsymbol{\eta} \circ \bar{u}_{k}\right|^{2} \leq\left|B_{-}^{k}\right|^{1-\frac{2}{2^{*}}}\left(\int_{B_{-}^{k}}\left|\bar{u}_{k}^{-} \ominus \boldsymbol{\eta} \circ \bar{u}_{k}\right|^{2^{*}}\right)^{\frac{2}{2^{*}}} \\
& \leq C\left|B_{-}^{k}\right|^{1-\frac{2}{2^{*}}} E_{k}=o\left(E_{k}\right) .
\end{aligned}
$$

We used in the last line Poincaré's inequality for $\bar{u}_{k}^{-}$that is vanishing on a set of uniformly positive measure. Now we can apply the concentration compactness lemma, [DLS14, Proposition 4.3], to the sequence $E_{k}^{-\frac{1}{2}} \bar{u}_{k}^{+}$and deduce the existence of translating sheets

$$
\bar{h}_{k}=\sum_{j} \llbracket y_{j, k} \oplus h_{j} \rrbracket
$$

with maps $h_{j} \in W^{1,2}\left(B_{\frac{5}{2}}, \mathcal{A}_{Q_{j}}\left(\mathbb{R}^{\bar{n}}\right)\right)$ and points $y_{j, k} \in \mathbb{R}^{\bar{n}}$ such that the following properties are satisfied:

$$
\begin{aligned}
& \left\|\mathcal{G}_{s}\left(E_{k}^{-\frac{1}{2}} \bar{u}_{k}^{+}, \bar{h}_{k}\right)\right\|_{2} \rightarrow 0, \\
& \liminf _{k \rightarrow \infty}\left(\int_{B_{\frac{5}{2}} \cap K_{k}} E_{k}^{-1}\left|D \bar{u}_{k}^{+}\right|^{2}-\int_{B_{\frac{5}{2}}}\left|D \bar{h}_{k}\right|^{2}\right) \geq 0, \\
& \underset{k \rightarrow \infty}{\limsup _{k}} \int_{B_{\frac{5}{2}}}\left(E_{k}^{-\frac{1}{2}}\left|D \bar{u}_{k}^{+}\right|-\left|D \bar{h}_{k}\right|\right)^{2} \leq \limsup _{k \rightarrow \infty}\left(E_{k}^{-1} \operatorname{Dir}\left(\bar{u}_{k}^{+}, B_{\frac{5}{2}}\right)-\operatorname{Dir}\left(\bar{h}_{k}, B_{\frac{5}{2}}\right)\right) .
\end{aligned}
$$

13.2 Lipschitz approximation of the competitors to $\bar{h}$ and $\boldsymbol{h}_{j}$. We fix a radius $s<\frac{5}{2}$.

To be able to interpolate later between $\bar{h}\left(\bar{h}_{k}\right)$ and $\bar{u}_{k}$ and similarly between the currents $T_{k}$ and $\mathbf{G}_{u_{k}}$, by using a Fubini type argument we may fix $s<t<\frac{5}{2}$ such that for some $C>0$ depending on $\frac{5}{2}-s$ we have

$$
\begin{aligned}
& \limsup _{k \rightarrow \infty} \int_{\partial B_{t}} \frac{\mathcal{G}_{s}\left(E_{k}^{-\frac{1}{2}} \bar{u}_{k}, \bar{h}\right)^{2}}{\left\|\mathcal{G}_{s}\left(E_{k}^{-\frac{1}{2}} \bar{u}_{k}, \bar{h}\right)\right\|_{2}^{2}}+E_{k}^{-1}\left|D \bar{u}_{k}\right|^{2}+|D \bar{h}|^{2} \leq C \quad \text { in case } b>0, \\
& \limsup _{k \rightarrow \infty} \int_{\partial B_{t}} \frac{\mathcal{G}_{s}\left(E_{k}^{-\frac{1}{2}} \bar{u}_{k}, \bar{h}_{k}\right)^{2}}{\left\|\mathcal{G}_{s}\left(E_{k}^{-\frac{1}{2}} \bar{u}_{k}, \bar{h}_{k}\right)\right\|_{2}^{2}}+E_{k}^{-1}\left|D \bar{u}_{k}\right|^{2}+\left|D \bar{h}_{k}\right|^{2} \leq C \quad \text { in case } b=0,
\end{aligned}
$$




$$
\mathbf{M}^{p}\left(\left\langle T_{k}-\boldsymbol{G}_{u_{k}}, f, t\right\rangle\right) \leq C \mathbf{M}^{p}\left(\left(T_{k}-\boldsymbol{G}_{u_{k}}\right)\left\llcorner\mathbf{C}_{3}\right) \leq C E_{k}^{1-2 \beta},\right.
$$

where, in (13.15), $f$ is the function defined by $f(y, z):=|y|$ for $(y, z) \in \pi_{0} \times \pi_{0}^{\perp}$. Also, in (13.14) we identified as before $\bar{h}_{k}$ with the map $\left(\bar{h}_{k}, 1\right)$ taking values in $\mathscr{A}_{Q}\left(\mathbb{R}^{\bar{n}}\right)$ and used (13.10); in (13.15) we used the conclusions of Proposition 9.6 as well as the Taylor expansion in [DLHMS, Equation (13.5)].

Now let us fix an arbitrary $\varepsilon>0$.

Case $b>0$ : Given any competitor $\bar{c} \in W^{1,2}\left(B_{\frac{5}{2}}, \mathscr{A}_{Q}\left(\mathbb{R}^{\bar{n}}\right)\right)$ to $\bar{h}$ that agrees with $\bar{h}$ outside of $B_{s}$, we may apply the Lipschitz approximation Lemma for special multivalued maps [DLHMS, Lemma 5.5] to $\bar{h}$ and $\bar{c}$ in order to obtain Lipschitz continuous maps $\bar{h}^{\varepsilon}$ and $\bar{c}^{\varepsilon}$ for which the inequalities [DLHMS, Equations (5.20) \& (5.21)] hold true with $\varepsilon^{2}$ in place of $\varepsilon$.

Case $b=0$ : We apply the same procedure as in the case of $b>0$. Given competitors $c_{j} \in W^{1,2}\left(B_{\frac{5}{2}}, \mathscr{A}_{Q_{j}}\left(\mathbb{R}^{\bar{n}}\right)\right)$ to $h_{j}$ that agree with $h_{j}$ outside of $B_{s}$ we may apply the Lipschitz approximation lemma to each $h_{j}$ and $c_{j}$ in order to obtain Lipschitz continuous maps $h_{j}^{\varepsilon}$ and $c_{j}^{\varepsilon}$ such that the inequalities [DLHMS, Equations $(5.20) \&(5.21)]$ hold true with $\varepsilon^{2}$ in place of $\varepsilon$. Furthermore we define

$$
\begin{aligned}
\bar{h}_{k}^{\varepsilon} & :=\sum_{j} \llbracket y_{j, k} \oplus h_{j}^{\varepsilon} \rrbracket, \\
\bar{c}_{k}^{\varepsilon} & :=\sum_{j} \llbracket y_{j, k} \oplus c_{j}^{\varepsilon} \rrbracket .
\end{aligned}
$$

13.3 Interpolating functions. The argument below does not distinguish between the cases $b>0, b=0$. To handle them simultaneously, we just consider the trivial sequence $\bar{h}_{k}=\bar{h}$ in the case when $b>0$.

For each $k$ we fix now an interpolating map $\varphi_{k} \in W^{1,2}\left(B_{t} \backslash B_{(1-\varepsilon) t}, \mathscr{A}_{Q}\left(\mathbb{R}^{\bar{n}}\right)\right)$ by means of Luckhaus' Lemma [DLHMS, Lemma 5.4] such that

$$
\begin{aligned}
& \varphi_{k}(x)=E_{k}^{-\frac{1}{2}} \bar{u}_{k}(x) \text { and } \varphi_{k}((1-\varepsilon) x)=\bar{h}_{k}^{\varepsilon}(x) \text { for all } x \in \partial B_{t}, \\
& \int_{B_{t} \backslash B_{(1-\varepsilon) t}}\left|D \varphi_{k}\right|^{2} \leq C \varepsilon\left(\int_{\partial B_{t}} E_{k}^{-1}\left|D \bar{u}_{k}\right|^{2}+\left|D \bar{h}_{k}^{\varepsilon}\right|^{2}\right)+\frac{C}{\varepsilon} \int_{\partial B_{t}} \mathcal{G}_{s}\left(E_{k}^{-\frac{1}{2}} \bar{u}_{k}, \bar{h}_{k}^{\varepsilon}\right)^{2} .
\end{aligned}
$$

Observe that by our choice of the Lipschitz approximation $\bar{h}_{k}^{\varepsilon}$ we have

$$
\int_{B_{t} \backslash B_{(1-\varepsilon) t}}\left|D \varphi_{k}\right|^{2} \leq C \varepsilon \quad \text { for large } k \text { (depending on } \varepsilon \text { ). }
$$

Moreover, observe that, by construction, $\lim \sup _{k \rightarrow \infty} \operatorname{Lip}\left(\bar{h}_{k}^{\varepsilon}\right) \leq C_{\varepsilon}^{*}$, where $C_{\varepsilon}^{*}$ is a constant depending on $\varepsilon$ but independent of $k$. Also, again for large values of $k$ (depending on our fixed $\varepsilon$ ):

$$
\begin{aligned}
\left\|\mathcal{G}_{s}\left(E_{k}^{-1 / 2} \bar{u}_{k}, \bar{h}_{k}^{\varepsilon}\right)\right\|_{L^{\infty}\left(\partial B_{t}\right)} & \leq C\left\|\mathcal{G}_{s}\left(E_{k}^{-1 / 2} \bar{u}_{k}, \bar{h}_{k}^{\varepsilon}\right)\right\|_{L^{2}\left(\partial B_{t}\right)}+C \operatorname{Lip}\left(E_{k}^{-1 / 2} \bar{u}_{k}\right)+C \operatorname{Lip}\left(\bar{h}_{k}^{\varepsilon}\right) \\
& \leq C \varepsilon+C E_{k}^{\beta-1 / 2}+C_{\varepsilon}^{*} .
\end{aligned}
$$


Hence, from [DLHMS, Equation (5.19)] we conclude that

$$
\operatorname{Lip}\left(\varphi_{k}\right) \leq C_{\varepsilon} E_{k}^{\beta-1 / 2}+C_{\varepsilon} \leq C_{\varepsilon} E_{k}^{\beta-1 / 2}
$$

where the last inequality is a consequence of the fact that $E_{k}^{\beta-1 / 2} \rightarrow \infty$ as $k \uparrow \infty$.

In particular we can define competitors to $E_{k}^{-\frac{1}{2}} \bar{u}_{k}$ on $B_{t}$ by

$$
\hat{c}_{k}(x):= \begin{cases}\varphi_{k}(x) & \text { for }(1-\varepsilon) t \leq|x| \leq t \\ \bar{c}_{k}^{\varepsilon}\left(\frac{x}{1-\varepsilon}\right) & \text { for }|x| \leq(1-\varepsilon) t\end{cases}
$$

We observe that by our construction we have

$$
\liminf _{k \rightarrow \infty} E_{k}^{-1} \operatorname{Dir}\left(\bar{u}_{k}, B_{t} \cap K_{k}\right)-\operatorname{Dir}\left(\hat{c}_{k}, B_{t}\right) \geq\left(\sum_{j} \operatorname{Dir}\left(h_{j}, B_{t}\right)-\operatorname{Dir}\left(c_{j}, B_{t}\right)\right)-C \varepsilon
$$

We have used (13.11), the closeness of the Dirichlet energies of $c_{j}$ and $c_{j}^{\varepsilon}$ and (13.16). As we have seen in the calculations below point (ii) above, we can use the fact that $\left\|D \Psi_{k}\right\|_{0}+\left\|D^{2} \Psi_{k}\right\|_{0}=O\left(E_{k}^{\frac{1}{2}}\right)$ to pass to $u_{k}$ and $w_{k}=\left(E_{k}^{\frac{1}{2}} \hat{c}_{k}, \Psi_{k}\left(\cdot, E_{k}^{\frac{1}{2}} \hat{c}_{k}\right)\right)$ still satisfying

$$
\liminf _{k \rightarrow \infty} E_{k}^{-1}\left(\operatorname{Dir}\left(u_{k}, B_{t} \cap K_{k}\right)-\operatorname{Dir}\left(w_{k}, B_{t}\right)\right) \geq\left(\sum_{j} \operatorname{Dir}\left(h_{j}, B_{t}\right)-\operatorname{Dir}\left(c_{j}, B_{t}\right)\right)-C \varepsilon .
$$

13.4 Interpolating Currents. By our choice of $t$, (13.15), and the fact that the boundary operator commutes with slicing we have

$$
\partial^{p}\left\langle T_{k}-\boldsymbol{G}_{u_{k}}, f, t\right\rangle=0 .
$$

Using [Fed69, $(4.2 .10)^{\nu}$ ], we can fix an isoperimetric filling $S_{k}$, which can be assumed to be representative $\bmod (p)$, such that

$$
\partial S_{k}=\left\langle T_{k}-\boldsymbol{G}_{u_{k}}, f, t\right\rangle \bmod (p)
$$

and

$$
\mathbf{M}\left(S_{k}\right)=\mathbf{M}^{p}\left(S_{k}\right) \leq C \mathbf{M}^{p}\left(\left\langle T_{k}-\boldsymbol{G}_{u_{k}}, f, t\right\rangle\right)^{\frac{m}{m-1}} \leq C E_{k}^{\frac{m(1-2 \beta)}{m-1}}=o\left(E_{k}\right)
$$

by the choice of $\beta$. 
13.5 Dirichlet minimality. We can now finally define a competitor to $T_{k}$ by

$$
Z_{k}:=T_{k}\left\llcorner\left(\mathbf{C}_{4} \backslash \mathbf{C}_{t}\right)+S_{k}+\boldsymbol{G}_{w_{k}} .\right.
$$

Observe that, by the hypotheses on $T_{k}$, Lemma 6.1 , and the choice of $S_{k}$, we have

$$
\partial^{p} Z_{k}=-\left[\left\langle T_{k}, f, t\right\rangle\right]+\left[\left\langle T_{k}-\mathbf{G}_{u_{k}}, f, t\right\rangle\right]+\left[\left\langle\mathbf{G}_{u_{k}}, f, t\right\rangle\right]=0 .
$$

Let us observe that by construction, and using once again the Taylor expansion of the mass of a special multi-valued graph [DLHMS, Equation (13.5)], we compute:

$$
\begin{aligned}
\mathbf{e}_{T_{k}}\left(B_{t}\right)-\frac{1}{2} \operatorname{Dir}\left(u_{k}, B_{t} \cap K_{k}\right) & =\mathbf{e}_{T_{k}}\left(B_{t} \backslash K_{k}\right)+o\left(E_{k}\right), \\
\mathbf{e}_{Z_{k}}\left(B_{t}\right)-\frac{1}{2} \operatorname{Dir}\left(w_{k}, B_{t}\right) & \leq \mathbf{M}\left(S_{k}\right)+\mathbf{e}_{\boldsymbol{G}_{w_{k}}}\left(B_{t}\right)-\frac{1}{2} \operatorname{Dir}\left(w_{k}, B_{t}\right) \leq o\left(E_{k}\right),
\end{aligned}
$$

where in the last equality we have used that $\operatorname{Dir}\left(w_{k}, B_{t}\right)=O\left(E_{k}\right)$ whereas $\operatorname{Lip}\left(w_{k}\right) \leq$ $C_{\varepsilon} E_{k}^{\beta}$, so that

$$
\mathbf{e}_{\mathbf{G}_{w_{k}}}-\frac{1}{2} \operatorname{Dir}\left(w_{k}, B_{t}\right)=\int_{B_{t}} \sum_{i} \bar{R}_{4}\left(D w_{k}^{i}\right) \leq C E_{k}^{1+2 \beta}=o\left(E_{k}\right) \quad \text { as } k \uparrow \infty .
$$

By minimality of $T_{k}$ in $\mathbf{C}_{3}$ we then have

$$
\begin{aligned}
0 & \geq \mathbf{M}\left(T_{k}\left\llcorner\mathbf{C}_{3}\right)-\mathbf{M}\left(Z_{k}\left\llcorner\mathbf{C}_{3}\right)\right.\right. \\
& =\mathbf{e}_{T_{k}}\left(B_{t}\right)-\mathbf{e}_{Z_{k}}\left(B_{t}\right) \\
& \geq \frac{1}{2}\left(\operatorname{Dir}\left(u_{k}, B_{t} \cap K_{k}\right)-\operatorname{Dir}\left(w_{k}, B_{t}\right)\right)+\mathbf{e}_{T_{k}}\left(B_{t} \backslash K_{k}\right)-o\left(E_{k}\right) .
\end{aligned}
$$

Hence dividing by $E_{k}$ and taking the $\lim \sup$ as $k \rightarrow \infty$ we deduce by (13.19)

$$
0 \geq \frac{1}{2}\left(\sum_{j} \operatorname{Dir}\left(h_{j}, B_{t}\right)-\operatorname{Dir}\left(c_{j}, B_{t}\right)\right)+\limsup _{k \rightarrow \infty} E_{k}^{-1} \mathbf{e}_{T_{k}}\left(B_{t} \backslash K_{k}\right) .
$$

Since $\varepsilon$ is arbitrary:

(i) Choosing $c_{j}=h_{j}$, we see that $\lim \sup _{k \rightarrow \infty} E_{k}^{-1} \mathbf{e}_{T_{k}}\left(B_{t} \backslash K_{k}\right)=0$.

(ii) By the arbitrariness of $c_{j}$ we conclude the Dirichlet minimality of $h_{j}$. Afterwards by (13.11) we deduce that $\limsup _{k \rightarrow \infty} E_{k}^{-1} \operatorname{Dir}\left(u_{k}, B_{t} \cap K_{t}\right)-\operatorname{Dir}\left(h_{k}, B_{t}\right)=$ 0 . In combination with (13.12) we obtain the second part of (ii), thus completing the proof. 


\section{Improved excess estimate and higher integrability}

So far, Proposition 9.6 and Theorem 13.3 have shown that if $T$ is as in Assumption 9.1 then there is a Lipschitz continuous multiple valued function (possibly special, in case $p$ is an even integer and $Q=\frac{p}{2}$ ) whose graph coincides with the current in a region where the excess measure is suitably small in a uniform sense; furthermore, if $T$ is also area minimizing $\bmod (p)$ then such an approximating Lipschitz multiple valued function is almost Dirichlet minimizing, and both the Dirichlet energy of the approximating function and the excess of the original current in the "bad region" decay faster than the excess. The goal of this section is to exploit the closeness of the Lipschitz approximation to a Dir-minimizer in order to deduce extra information concerning the behavior of the excess measure of $T$. We begin observing that the classical result on the higher integrability of the gradient of a harmonic function extends not only to classical multiple valued functions, as it is shown in [DLS14, Theorem 6.1], but also to special multiple valued functions.

Theorem 14.1. There exists $p>2$ such that for every $\Omega^{\prime} \Subset \Omega \subset \mathbb{R}^{m}$ open domains, there is a constant $C>0$ such that

$$
\|D u\|_{L^{p}\left(\Omega^{\prime}\right)} \leq C\|D u\|_{L^{2}(\Omega)} \text { for every Dir-minimizing } u \in W^{1,2}\left(\Omega, \mathscr{A}_{Q}\left(\mathbb{R}^{n}\right)\right) .
$$

Proof. The proof is the very same presented in [DLS14, Theorem 6.1]: one only has to replace the Almgren embedding $\boldsymbol{\xi}$ for $\mathcal{A}_{Q}\left(\mathbb{R}^{n}\right)$ used in there with the new version of the Almgren embedding $\zeta$ for $\mathscr{A}_{Q}\left(\mathbb{R}^{n}\right)$ introduced in [DLHMS, Theorem 5.1].

As a direct corollary of the first harmonic approximation and the higher integrability of the gradient we obtain the following result.

Corollary 14.2. For every $\eta>0$ there exist an $\varepsilon>0$ and a constant $C>0$ with the property that, if $T$ satisfies Assumption 9.1 and is area minimzing $\bmod (p)$ in the cylinder $\mathbf{C}_{4 s}(x)$ with $E \leq \varepsilon$ then for every $A \subset B_{s}$ with $\left|A \cap B_{s}\right| \leq \varepsilon\left|B_{s}\right|$ we have

$$
\mathbf{e}_{T}(A) \leq\left(\eta E+C \mathbf{A}^{2} s^{2}\right) s^{m} .
$$

Proof. By scaling and translating we may assume without loss of generality that $x=0$ and $s=1$. We fix $\beta=\frac{1}{4 m}$ and $\eta_{*}>0$ to be determined below. Now let $\varepsilon_{*}=\varepsilon_{*}\left(\beta, \eta_{*}\right)$ taken from Theorem 13.3. We distinguish the following two cases: either $\mathbf{A} \leq \varepsilon_{*} E^{\frac{1}{2}}$ or $\mathbf{A}>\varepsilon_{*} E^{\frac{1}{2}}$. In the latter case the inequality holds trivially with $C=\varepsilon_{*}^{-2}$ because

$$
\mathbf{e}_{T}(A) \leq E \leq \varepsilon_{*}^{-2} \mathbf{A}^{2} .
$$

In the first case, we can apply the first harmonic approximation, Theorem 13.3. Now let $h(x)=(\bar{h}(x), \Psi(x, \bar{h}(x)))$, with $\bar{h}$ Dirichlet minimizing, the associated map as in (i). By (13.3) we directly conclude that

$$
\mathbf{e}_{T}(A \backslash K) \leq \eta_{*} E,
$$


where $K$ is, as usual, the "good set" for the $E^{\beta}$-Lipschitz approximation of $T$ in $\mathbf{C}_{3}$ as in Proposition 9.6. In order to estimate the $\mathbf{e}_{T}$ measure of the portion of $A$ inside $K$, we observe that

$$
\begin{aligned}
\left.\left|\mathbf{e}_{T}(A \cap K)-\frac{1}{2} \int_{A \cap K}\right| D h\right|^{2} \mid & =\left.\left|\mathbf{e}_{G_{u}}(A \cap K)-\frac{1}{2} \int_{A \cap K}\right| D h\right|^{2} \mid \\
& \leq\left.\left|\mathbf{e}_{G_{u}}(A \cap K)-\frac{1}{2} \int_{A \cap K}\right| D u\right|^{2}\left|+\frac{1}{2}\right| \int_{A \cap K}|D u|^{2}-|D h|^{2} \mid \\
& =: I+I I .
\end{aligned}
$$

The first addendum can be bounded by the Taylor expansion of mass by

$$
I \leq C \operatorname{Lip}(u)^{2} \int_{A \cap K}|D u|^{2} \leq C E^{1+2 \beta}
$$

the second can be estimated using (13.5) and $|D u|^{2}-|D h|^{2}=(|D u|+|D h|)(|D u|-$ $|D h|)$ by

$$
I I \leq C\left(\int_{A \cap K}|D u|^{2}+|D h|^{2}\right)^{\frac{1}{2}}\left(\int_{A \cap K}(|D u|-|D h|)^{2}\right)^{\frac{1}{2}} \leq C \eta_{*}^{\frac{1}{2}} E .
$$

Recall that $\mathbf{A} \leq \varepsilon_{*} E^{\frac{1}{2}}$ implies that $\|D \Psi\| \leq C E^{\frac{1}{2}}$. Hence we have

$$
\begin{aligned}
\int_{A \cap K}|D h|^{2} & =\int_{A \cap K}|D \bar{h}|^{2}+\left|D_{x} \Psi(x, \bar{h})+D_{y} \Psi(x, \bar{h}) D \bar{h}\right|^{2} \\
& \leq(1+C E) \int_{A \cap K}|D \bar{h}|^{2}+C E|A \cap K| .
\end{aligned}
$$

Using the higher integrability for Dirichlet minimizers we can estimate further

$$
\begin{aligned}
\int_{A \cap K}|D \bar{h}|^{2} & \leq|A \cap K|^{1-\frac{2}{p}}\left(\int_{A \cap K}|D \bar{h}|^{p}\right)^{\frac{2}{p}} \\
& \leq C|A \cap K|^{1-\frac{2}{p}} \int_{B_{2}}|D \bar{h}|^{2} \leq C|A \cap K|^{1-\frac{2}{p}} E .
\end{aligned}
$$

Collecting all the estimates we get in conclusion

$$
\begin{aligned}
\mathbf{e}_{T}(A) & \leq \mathbf{e}_{T}(A \backslash K)+\left.\left.\left|\mathbf{e}_{T}(A \cap K)-\frac{1}{2} \int_{A \cap K}\right| D h\right|^{2}\left|+\frac{1}{2} \int_{A \cap K}\right| D h\right|^{2} \\
& \leq\left(\eta_{*}+C E^{2 \beta}+C \eta_{*}^{\frac{1}{2}}+C|A \cap K|^{1-\frac{2}{p}}\right) E .
\end{aligned}
$$

Hence, the estimate in (14.1) follows also in this case after suitably choosing $\varepsilon$ and $\eta_{*}$ depending on $\eta$. 
For the following proof, we introduce the centered maximal function for a general radon measure $\mu$ on $\mathbb{R}^{m}$ by setting

$$
\mathbf{m}_{c} \mu(x):=\sup _{s \geq 0} \frac{\mu\left(B_{s}(x)\right)}{\omega_{m} s^{m}} .
$$

Observe that one has the straightforward comparison between the centered and non-centered maximal functions

$$
\mathbf{m}_{c} \mu(x) \leq \mathbf{m} \mu(x) \leq 2^{m} \mathbf{m}_{c} \mu(x) .
$$

Although the two quantities are therefore comparable, we decided to work for this proof with the centered version since in our opinion the geometric idea becomes more easily accessible. Furthermore we note that since the map $x \mapsto \frac{\mu\left(B_{s}(x)\right)}{\omega_{m} s^{m}}$ is lower semicontinuous, $x \mapsto \mathbf{m}_{c} \mu(x)$ is lower semicontinuous as it is the supremum of a family of lower semicontinuous functions.

Theorem 14.3. There exist constants $0<q<1, C, \varepsilon>0$ with the following property. If $T$ is area minimzing $\bmod (p)$ in the cylinder $\mathbf{C}_{4}$ and satisfies Assumption 9.1 with $E \leq \varepsilon$ then

$$
\int_{B_{2}}\left(\min \left\{\mathbf{m}_{c} \mathbf{e}, 1\right\}\right)^{q} d \mathbf{e} \leq C e^{C \mathbf{A}^{2}} E^{1+q} .
$$

In particular this implies the following estimate

$$
\int_{B_{2} \cap\left\{\mathbf{m}_{c} \mathbf{e} \leq 1\right\}}\left(\mathbf{m}_{c} \mathbf{e}\right)^{q} d \mathbf{e} \leq C e^{C \mathbf{A}^{2}} E^{1+q} .
$$

REMARK 14.4. Observe that the excess measure e can be decomposed as

$$
\mathbf{e}=\mathbf{d} \mathscr{L}^{m}+\mathbf{e}_{\text {sing }},
$$

where $\mathscr{L}^{m}$ denotes the Lebesgue measure in $\mathbb{R}^{m}, \mathbf{e}_{\text {sing }} \perp \mathscr{L}^{m}$ and $\mathbf{d}$ is the excess density as in Definition 9.2. Since $\mathbf{d}(x) \leq \mathbf{m}_{c} \mathbf{e}(x)$ for every $x \in B_{2}$, we have

$$
\int_{B_{2}}\left(\min \left\{\mathbf{m}_{c} \mathbf{e}, 1\right\}\right)^{q} d \mathbf{e} \geq \int_{B_{2}}(\min \{\mathbf{d}, 1\})^{q} d \mathbf{e} \geq \int_{B_{2}}(\min \{\mathbf{d}, 1\})^{q} \mathbf{d} d x,
$$

so that formula (14.3) in particular implies the following higher integrability of the excess density:

$$
\int_{\{\mathbf{d} \leq 1\} \cap B_{2}} \mathbf{d}^{1+q} d x \leq C e^{C \mathbf{A}^{2}} E^{1+q} \leq C E^{1+q} .
$$

Proof. Let us first observe that given any measure $\mu$ on $\mathbb{R}^{m}$ we have that, for any fixed $r>0$ and $t>0$, if

$$
\frac{\mu\left(B_{s}(x)\right)}{\omega_{m} s^{m}} \leq\left(\frac{3}{4}\right)^{m} t \quad \forall s \geq 4 r
$$


then for some constant $C$ depending on $m$ we have

$$
\left|B_{r}(x) \cap\left\{y: \mathbf{m}_{c} \mu(y)>t\right\}\right| \leq \frac{C}{t} \mu\left(B_{4 r}(x) \cap\left\{y: \mathbf{m}_{c} \mu(y)>\frac{t}{2}\right\}\right) .
$$

This can be seen as follows: we first note that for $y \in B_{r}(x)$ we have

$$
\frac{\mu\left(B_{s}(y)\right)}{\omega_{m} s^{m}} \leq \begin{cases}\left(\frac{4 r}{s}\right)^{m} \frac{\mu\left(B_{4 r}(x)\right)}{\omega_{m}(4 r)^{m}} & \text { if } s+|x-y| \leq 4 r \\ \left(\frac{s+|x-y|}{s}\right)^{m} \frac{\mu\left(B_{s+|x-y|}(x)\right)}{\omega_{m}(s+|x-y|)^{m}} & \text { if } s+|x-y| \geq 4 r .\end{cases}
$$

Hence, we deduce that if $s \geq 3 r$ then $\frac{\mu\left(B_{s}(y)\right)}{\omega_{m} s^{m}} \leq t$ : in other words, if $\frac{\mu\left(B_{s}(y)\right)}{\omega_{m} s^{m}}>t$ then we must have $B_{s}(y) \subset B_{4 r}(x)$. This implies that

$$
B_{r}(x) \cap\left\{y: \mathbf{m}_{c} \mu(y) \geq t\right\}=B_{r}(x) \cap\left\{y: \mathbf{m}_{c} \mu\left\llcorner_{B_{4 r}(x)}(y) \geq t\right\},\right.
$$

so that (14.5) follows by a variation of the classical maximal function estimate applied to $\mu\left\llcorner_{B_{4 r}(x)}{ }^{2}\right.$

Furthermore we recall that by classical differentiation theory of radon measures 3 one has as well

$$
\mu\left(B_{r}(x) \cap\left\{y: \mathbf{m}_{c} \mu(y) \leq t\right\}\right) \leq t\left|B_{r}(x) \cap\left\{y: \mathbf{m}_{c} \mu(y) \leq t\right\}\right| .
$$

In what follows, for the sake of simplicity, we will work with the measure $\mathbf{e}=$ $\mathbf{e}\left\llcorner B_{4}\right.$, which is defined on the whole $\mathbb{R}^{m}$.

Step 1: For every $\eta>0$ there exist positive constants $\lambda, \varepsilon, C$ with the property that if

$$
r:=\sup \left\{s: \frac{\mathbf{e}\left(B_{s}(x)\right)}{\omega_{m} s^{m}} \geq \frac{t}{\lambda}\right\} \quad \text { and } \quad \frac{t}{\lambda} \leq \varepsilon,
$$

then

$$
\begin{aligned}
\mathbf{e}( & \left.B_{r}(x) \cap\left\{y: \mathbf{m}_{c} \mathbf{e}(y)>t\right\}\right) \\
\leq & \left(2 \omega_{m}^{-1} \eta+C \mathbf{A}^{2}\left(\frac{2 \lambda}{t} \mathbf{e}\left(B_{r}(x) \cap\left\{y: \mathbf{m}_{c} \mathbf{e}(y)>\frac{t}{2 \lambda}\right\}\right)\right)^{\frac{m+2}{m}}\right) \\
& \mathbf{e}\left(B_{r}(x) \cap\left\{y: \mathbf{m}_{c} \mathbf{e}(y)>\frac{t}{2 \lambda}\right\}\right) .
\end{aligned}
$$

Proof of Step 1: Let $\eta>0$ be given, and let $\varepsilon>0$ be given by Corollary 14.2 in correspondence with this choice of $\eta$. Also fix $\lambda>\left(\frac{4}{3}\right)^{m}$. By the definition of $r$

2 The variation in use here can be deduced in a straightforward fashion from the classical estimate for the whole space: apply the classical estimate (see e.g. [Mat95, Theorem 2.19 (2)]) to the measure $\tilde{\mu}:=\mu\left\llcorner\left\{\mathbf{m}_{c} \mu>\frac{t}{2}\right\}\right.$ and note that since $\mu \leq \tilde{\mu}+\frac{t}{2} \mathscr{L}^{m}$ we have $\left\{\mathbf{m}_{c} \mu>t\right\} \subset\left\{\mathbf{m}_{c} \tilde{\mu}>\frac{t}{2}\right\}$.

3 Note for each $y \in B_{r}(x) \cap\left\{\mathbf{m}_{c} \mu \leq t\right\}$ one has $\liminf \operatorname{in}_{r \downarrow 0} \frac{\mu\left(B_{r}(y)\right)}{\left|B_{r}(y)\right|} \leq t$, hence (14.6) follows for instance from [Mat95, Lemma 2.13 (1)]. 
and the continuity of measures along increasing and decreasing sequence of sets, we easily see that

$$
\frac{\mathbf{e}\left(\overline{B_{r}(x)}\right)}{\omega_{m} r^{m}}=\frac{\mathbf{e}\left(B_{r}(x)\right)}{\omega_{m} r^{m}}=\frac{t}{\lambda}>\frac{\mathbf{e}\left(B_{s}(x)\right)}{\omega_{m} s^{m}} \text { for all } s>r .
$$

Thus we can apply (14.5) with $\mu=\mathbf{e}$, thus deducing that

$$
\left|B_{r}(x) \cap\left\{y: \mathbf{m}_{c} \mathbf{e}(y)>t\right\}\right| \leq \frac{C}{t} \mathbf{e}\left(B_{4 r}(x) \cap\left\{y: \mathbf{m}_{c} \mathbf{e}(y)>\frac{t}{2}\right\}\right) \leq \frac{C}{\lambda} \omega_{m}(4 r)^{m} .
$$

Since $\frac{t}{\lambda} \leq \varepsilon$, if we choose $\lambda \geq \frac{4^{m} C}{\varepsilon}$ then we can apply Corollary 14.2, which, together with (14.9), yields

$$
\begin{aligned}
\mathbf{e}\left(B_{r}(x) \cap\left\{y: \mathbf{m}_{c} \mathbf{e}(y)>t\right\}\right) & \leq \omega_{m}^{-1} 4^{-m} \eta \mathbf{e}\left(B_{4 r}(x)\right)+C \mathbf{A}^{2} r^{m+2} \\
& \leq \omega_{m}^{-1} \eta \mathbf{e}\left(B_{r}(x)\right)+C \mathbf{A}^{2} r^{m+2} .
\end{aligned}
$$

Using (14.6) and (14.9), namely the identity $\frac{t}{\lambda} \omega_{m} r^{m}=\mathbf{e}\left(B_{r}(x)\right)$ we have

$$
\mathbf{e}\left(B_{r}(x) \cap\left\{y: \mathbf{m}_{c} \mathbf{e}(y) \leq \frac{t}{2 \lambda}\right\}\right) \leq \frac{t}{2 \lambda}\left|B_{r}(x)\right| \leq \frac{1}{2} \mathbf{e}\left(B_{r}(x)\right) .
$$

This implies that

$$
\omega_{m} r^{m}=\frac{\lambda}{t} \mathbf{e}\left(B_{r}(x)\right) \leq \frac{2 \lambda}{t} \mathbf{e}\left(B_{r}(x) \cap\left\{y: \mathbf{m}_{c} \mathbf{e}(y)>\frac{t}{2 \lambda}\right\}\right) .
$$

Using this estimate in (14.10) we deduce (14.8).

Step 2: For every $\eta>0$ there exist positive constants $\lambda, \varepsilon, C$ such that if

$$
4^{2 m} E \leq \frac{t}{\lambda} \leq \varepsilon \quad \text { and } \quad r \leq 3
$$

then, setting $\bar{r}:=r+4\left(\frac{\lambda E}{t}\right)^{\frac{1}{m}}$, we have

$$
\begin{aligned}
\mathbf{e} & \left(B_{r} \cap\left\{y: \mathbf{m}_{c} \mathbf{e}(y)>t\right\}\right) \\
& \leq c_{B}\left(\eta+C \mathbf{A}^{2}\left(\frac{2 \lambda E}{t}\right)^{\frac{m+2}{m}}\right) \mathbf{e}\left(B_{\bar{r}} \cap\left\{y: \mathbf{m}_{c} \mathbf{e}(y)>\frac{t}{2 \lambda}\right\}\right),
\end{aligned}
$$

where $c_{B}$ denotes the Besicovitch constant in $\mathbb{R}^{m}$.

Proof of Step 2: For each $x \in B_{r} \cap\left\{y: \mathbf{m}_{c} \mathbf{e}(y)>t\right\}$ we let

$$
r_{x}:=\sup \left\{s: \frac{\mathbf{e}\left(B_{s}(x)\right)}{\omega_{m} s^{m}} \geq \frac{t}{\lambda}\right\} .
$$

We must have $0<r_{x} \leq \frac{1}{4}$, since $\mathbf{m}_{c} \mathbf{e}(x)>t \geq t / \lambda$, and since for each $x \in B_{3}$ we have

$$
\frac{\mathbf{e}\left(B_{s}(x)\right)}{\omega_{m} s^{m}} \leq 4^{2 m} E \leq \frac{t}{\lambda} \quad \forall s \geq \frac{1}{4}
$$


We apply the Besicovitch covering theorem to the family

$$
\mathcal{B}:=\left\{\overline{B_{r_{x}}(x)}: x \in B_{r} \cap\left\{y: \mathbf{m}_{c} \mathbf{e}(y)>t\right\}\right\}
$$

and obtain sub-collections $\mathcal{B}_{1}, \ldots, \mathcal{B}_{c_{B}}$ of balls such that each subfamily is pairwise disjoint and

$$
B_{r} \cap\left\{y: \mathbf{m}_{c} \mathbf{e}(y)>t\right\} \subset \bigcup_{j=1}^{c_{B}} \bigcup_{B_{r_{x}}(x) \in \mathcal{B}_{j}} \overline{B_{r_{x}}(x)}
$$

Since for each of these balls we have $\omega_{m} r_{x}{ }^{m}=\frac{\lambda}{t} \mathbf{e}\left(B_{r_{x}}(x)\right) \leq \frac{\lambda}{t} E \omega_{m} 4^{m}$, we deduce $B_{r_{x}}(x) \subset B_{\bar{r}}$. Hence the result follows from

$$
\mathbf{e}\left(B_{r} \cap\left\{y: \mathbf{m}_{c} \mathbf{e}(y)>t\right\}\right) \leq \sum_{i=1}^{c_{B}} \sum_{B_{r_{x}}(x) \in \mathcal{B}_{i}} \mathbf{e}\left(B_{r_{x}}(x) \cap\left\{y: \mathbf{m}_{c} \mathbf{e}(y)>t\right\}\right)
$$

where we used that by Step $1 \mathbf{e}\left(\partial B_{r_{x}}(x)=0\right.$ for each of these balls, and then applying (14.8) of Step 1 to each.

Step 3: For every $\eta>0$ there are constants $C, \lambda, \varepsilon$ such that for every $k \geq 2$ with

$$
(2 \lambda)^{k} E \leq \varepsilon \quad \text { and } \quad r \leq \frac{5}{2}
$$

we have

$$
\mathbf{e}\left(B_{r} \cap\left\{y: \mathbf{m}_{c} \mathbf{e}(y)>(2 \lambda)^{k} E\right\}\right) \leq\left(c_{B} \eta\right)^{k} e^{C \mathbf{A}^{2}} \mathbf{e}\left(B_{r+\frac{1}{2}} \cap\left\{y: \mathbf{m}_{c} \mathbf{e}(y)>2 \lambda E\right\}\right) .
$$

Proof of Step 3: This is obtained by iterating Step 2. More precisely, for each $2 \leq l \leq k$ we set

$$
t_{l}:=(2 \lambda)^{l} E, \quad\left\{\begin{array}{l}
r_{k}:=r \\
r_{l-1}:=r_{l}+4\left(\frac{\lambda E}{t_{l}}\right)^{\frac{1}{m}}=r_{l}+\frac{4 \lambda^{\frac{1}{m}}}{(2 \lambda)^{\frac{l}{m}}} \quad \text { for } 2 \leq l \leq k-1 .
\end{array}\right.
$$

Using $f(r, t):=\mathbf{e}\left(B_{r} \cap\left\{y: \mathbf{m}_{c} \mathbf{e}(y)>t\right\}\right)$ and $c_{\mathbf{A}}:=\frac{C \mathbf{A}^{2}}{c_{B} \eta}$ we may write (14.11) as

$$
\begin{aligned}
f\left(r_{l}, t_{l}\right) & \leq c_{B} \eta\left(1+c_{\mathbf{A}}\left(\frac{2 \lambda E}{t_{l}}\right)^{\frac{m+2}{m}}\right) f\left(r_{l-1}, t_{l-1}\right) \\
& =c_{B} \eta\left(1+c_{\mathbf{A}}\left(\frac{1}{2 \lambda}\right)^{\frac{m+2}{m}(l-1)}\right) f\left(r_{l-1}, t_{l-1}\right) .
\end{aligned}
$$


Now (14.12) is a consequence of the following estimates ( $\lambda$ is sufficient large)

$$
\begin{gathered}
r_{1}=r_{k}+4 \lambda^{\frac{1}{m}} \sum_{l=2}^{k}(2 \lambda)^{-\frac{l}{m}} \leq r+4 \lambda^{\frac{1}{m}} \sum_{l=2}^{\infty}(2 \lambda)^{-\frac{l}{m}} \leq r+\frac{1}{2}, \\
\prod_{l=2}^{k} c_{B} \eta\left(1+c_{\mathbf{A}}\left(\frac{1}{2 \lambda}\right)^{\frac{m+2}{m}(l-1)}\right) \leq\left(c_{B} \eta\right)^{k} e^{C c_{\mathbf{A}}} .
\end{gathered}
$$

In particular, the first estimates ensures that we may apply step 2 for each pair $\left(t_{l}, r_{l}\right)$.

Conclusion: First we fix $\eta>0$ sufficiently small, so that $c_{B} \eta<1$, and afterwards $q>0$ such that $a:=(2 \lambda)^{q} c_{B} \eta<1$. Now we observe that with $(2 \lambda)^{k_{0}} E \leq \varepsilon \lambda<$ $(2 \lambda)^{k_{0}+1} E$ we have

$$
\begin{aligned}
& \int_{B_{2} \cap\left\{(2 \lambda)^{2} E<\mathbf{m}_{c} \mathbf{e}\right\}}\left(\min \left\{\mathbf{m}_{c} \mathbf{e}, \lambda \varepsilon\right\}\right)^{q} d \mathbf{e} \\
& \leq \sum_{k=2}^{k_{0}} \int_{B_{2} \cap\left\{(2 \lambda)^{k} E<\mathbf{m}_{c} \mathbf{e} \leq(2 \lambda)^{k+1} E\right\}}\left(\mathbf{m}_{c} \mathbf{e}\right)^{q} d \mathbf{e}+\int_{B_{2} \cap\left\{(2 \lambda)^{k_{0}+1} E<\mathbf{m}_{c} \mathbf{e}\right\}}\left((2 \lambda)^{k_{0}+1} E\right)^{q} d \mathbf{e} \\
& \leq(4 \lambda)^{q} E^{q} \sum_{k=2}^{k_{0}+1}(2 \lambda)^{q k} \mathbf{e}\left(B_{2} \cap\left\{\mathbf{m}_{c} \mathbf{e}>(2 \lambda)^{k} E\right\}\right) \leq(4 \lambda)^{q} E^{q} e^{C \mathbf{A}^{2}} E\left(\omega_{m} 4^{m}\right) \sum_{k=2}^{k_{0}+1} a^{k} \\
& \leq C e^{C \mathbf{A}^{2}} E^{1+q} .
\end{aligned}
$$

Combining this with

$$
\int_{B_{2} \cap\left\{\mathbf{m}_{c} \mathbf{e} \leq(2 \lambda)^{2} E\right\}}\left(\mathbf{m}_{c} \mathbf{e}\right)^{q} d \mathbf{e} \leq(2 \lambda)^{2 q} E^{q} \mathbf{e}\left(B_{2} \cap\left\{\mathbf{m}_{c} \mathbf{e} \leq 2 \lambda E\right\}\right) \leq C E^{1+q}
$$

proves the result, modulo choosing a smaller value for $\varepsilon$.

\section{Almgren's strong approximation theorem}

We can finally state and prove the main Lipschitz approximation result for area minimizing currents $\bmod (p)$, which contains improved estimates with respect to Proposition 9.6.

Theorem 15.1 (Almgren's strong approximation). There exist constants $\varepsilon, \gamma, C>$ 0 (depending on $m, \bar{n}, n, Q$ ) with the following property. Let $T$ be as in Assumption 9.1 in the cylinder $\mathbf{C}_{4 r}(x)$, and assume it is area $\operatorname{minimizing} \bmod (p)$. Also assume that $E=\mathbf{E}\left(T, \mathbf{C}_{4 r}(x)\right)<\varepsilon$. Then, there are $u: B_{r}(x) \rightarrow \mathcal{A}_{Q}\left(\mathbb{R}^{n}\right)$ if $Q<\frac{p}{2}$, or $u: B_{r}(x) \rightarrow \mathscr{A}_{Q}\left(\mathbb{R}^{n}\right)$ if $Q=\frac{p}{2}$, and a closed set $K \subset B_{r}(x)$ such that:

$$
\operatorname{Gr}(u) \subset \Sigma,
$$




$$
\begin{gathered}
\operatorname{Lip}(u) \leq C\left(E+\mathbf{A}^{2} r^{2}\right)^{\gamma} \quad \text { and } \quad \operatorname{osc}(u) \leq C \mathbf{h}\left(T, \mathbf{C}_{4 r}(x), \pi_{0}\right)+C r\left(E^{1 / 2}+r \mathbf{A}\right), \\
\mathbf{G}_{u}\left\llcorner\left(K \times \mathbb{R}^{n}\right)=T\left\llcorner\left(K \times \mathbb{R}^{n}\right) \bmod (p)\right.\right. \\
\left|B_{r}(x) \backslash K\right| \leq\|T\|\left(\left(B_{r}(x) \backslash K\right) \times \mathbb{R}^{n}\right) \leq C\left(E+r^{2} \mathbf{A}^{2}\right)^{1+\gamma} r^{m}, \\
\left.\left|\|T\|\left(\mathbf{C}_{\sigma r}(x)\right)-Q \omega_{m}(\sigma r)^{m}-\frac{1}{2} \int_{B_{\sigma r}}\right| D u\right|^{2} \mid \leq\left(E+r^{2} \mathbf{A}^{2}\right)^{1+\gamma} r^{m} \quad \forall 0<\sigma<1 .
\end{gathered}
$$

The key improvement with respect to the conclusions of Proposition 9.6 lies in the superlinear power of the excess in (15.4) and (15.5). In turn, this gain is a consequence of the following improved excess estimate, analogous to [DLS14, Theorem 7.1].

Theorem 15.2 (Almgren's strong excess estimate). There exist constants $\varepsilon_{*}, \gamma_{*}, C>$ 0 (depending on $m, \bar{n}, n, Q$ ) with the following property. Assume $T$ satisfies Assumption 9.1 and is area minimizing $\bmod (p)$ in $\mathbf{C}_{4}$. If $E:=\mathbf{E}\left(T, \mathbf{C}_{4}\right)<\varepsilon_{*}$, then

$$
\mathbf{e}_{T}(A) \leq C\left(E^{\gamma_{*}}+|A|^{\gamma_{*}}\right)\left(E+\mathbf{A}^{2}\right) \quad \text { for every Borel } A \subset B_{9 / 8} .
$$

Let us assume for the moment the validity of Theorem 15.2, and let us then show how Theorem 15.1 follows.

Proof of Theorem 15.1. As usual, since the statement is scale-invariant, we may assume $x=0$ and $r=1$. Choose $\beta<\min \left\{\frac{1}{2 m}, \frac{\gamma_{*}}{2\left(1+\gamma_{*}\right)}\right\}$, where $\gamma^{*}$ is given by Theorem 15.2. Let $u$ be the $E^{\beta}$-Lipschitz approximation of $T$, so that (15.1) and (15.3) are an immediate consequence of Proposition 9.6. Also the estimates in (15.2) follow in a straightforward fashion if we choose $\gamma \leq \beta$ and we recall that $\|D \Psi\|_{0} \leq C\left(E^{1 / 2}+\mathbf{A}\right)$. Now we come to the proof of the volume estimate (15.4). Set $A:=\left\{\mathbf{m e}_{T}>E^{2 \beta}\right\} \cap B_{9 / 8}$. By (9.5), we have that $|A| \leq C E^{1-2 \beta}$. In order to improve the estimate, we use Almgren's strong excess estimate: indeed, equation (15.6) implies that

$$
\mathbf{e}_{T}(A) \leq C E^{\gamma_{*}}\left(1+E^{-2 \beta \gamma_{*}}\right)\left(E+\mathbf{A}^{2}\right),
$$

so that when we plug (15.7) back into (9.5) we have

$$
\begin{gathered}
\left|B_{1} \backslash K\right| \leq C E^{-2 \beta} \mathbf{e}_{T}(A) \leq C E^{\gamma_{*}-2 \beta\left(1+\gamma_{*}\right)}\left(1+E^{2 \beta \gamma_{*}}\right)\left(E+\mathbf{A}^{2}\right) \\
\leq C E^{\gamma_{*}-2 \beta\left(1+\gamma_{*}\right)}\left(E+\mathbf{A}^{2}\right)
\end{gathered}
$$

and the inequality

$$
\left|B_{1} \backslash K\right| \leq C\left(E+\mathbf{A}^{2}\right)^{1+\gamma}
$$


follows with $\min \left\{\gamma_{*}-2 \beta\left(1+\gamma_{*}\right), \beta\right\}>0$ because of our choice of $\beta$. (15.4) is then a simple consequence of

$$
\|T\|\left(\left(B_{1} \backslash K\right) \times \mathbb{R}^{n}\right) \leq \mathbf{e}_{T}\left(B_{1} \backslash K\right)+Q\left|B_{1} \backslash K\right|
$$

Finally, we take any $0<\sigma<1$ and we estimate:

$$
\begin{aligned}
& \left.\left|\|T\|\left(\mathbf{C}_{\sigma}(x)\right)-Q \omega_{m} \sigma^{m}-\frac{1}{2} \int_{B_{\sigma}}\right| D u\right|^{2} \mid \\
& \quad \leq \mathbf{e}_{T}\left(B_{\sigma} \backslash K\right)+\mathbf{e}_{\mathbf{G}_{u}}\left(B_{\sigma} \backslash K\right)+\left.\left|\mathbf{e}_{\mathbf{G}_{u}}\left(B_{\sigma}\right)-\frac{1}{2} \int_{B_{\sigma}}\right| D u\right|^{2} \mid \\
& \quad \stackrel{(15.7)}{\leq} C\left(E+\mathbf{A}^{2}\right)^{1+\gamma}+C\left|B_{\sigma} \backslash K\right|+C \operatorname{Lip}(u)^{2} \int_{B_{\sigma}}|D u|^{2} \\
& \quad \leq C\left(E+\mathbf{A}^{2}\right)^{1+\gamma} .
\end{aligned}
$$

We turn now to the proof of Theorem 15.2. We will use in an essential way the $\operatorname{minimality} \bmod (p)$ of $T$, and in order to do that we need to construct a suitable competitor. In this process, a key role will be played by the following result, analogous to [DLS14, Proposition 7.3]

Proposition 15.3. Let $\beta \in\left(0, \frac{1}{2 m}\right)$, and assume that $T$ satisfies Assumption 9.1 and is area minimizing $\bmod (p)$ in $\mathbf{C}_{4}$. Let $u$ be its $E^{\beta}$-Lipschitz approximation. Then, there exist constants $\varepsilon, \gamma, C>0$ and a subset of radii $B \subset[9 / 8,2]$ with measure $|B|>1 / 2$ with the following property. If $\mathbf{E}\left(T, \mathbf{C}_{4}\right)<\varepsilon$, then for every $\sigma \in B$ there exists a $Q$-valued map $g \in \operatorname{Lip}\left(B_{\sigma}, \mathcal{A}_{Q}\left(\mathbb{R}^{n}\right)\right)$ if $Q<\frac{p}{2}$ or $g \in \operatorname{Lip}\left(B_{\sigma}, \mathscr{A}_{Q}\left(\mathbb{R}^{n}\right)\right)$ if $Q=\frac{p}{2}$ such that

$$
\left.g\right|_{\partial B_{\sigma}}=\left.u\right|_{\partial B_{\sigma}}, \quad \operatorname{Lip}(g) \leq C\left(E+r^{2} \mathbf{A}^{2}\right)^{\beta}, \quad \operatorname{spt}(g(x)) \subset \Sigma \forall x \in B_{\sigma},
$$

and

$$
\int_{B_{\sigma}}|D g|^{2} \leq \int_{B_{\sigma} \cap K}|D u|^{2}+C\left(E+\mathbf{A}^{2}\right)^{1+\gamma} .
$$

Proof. The proof is obtained by a "regularization by convolution" procedure, analogous to that of [DLS14, Proposition 7.3], modulo using the embedding $\zeta$ of [DLHMS, Theorem 5.1] in place of $\boldsymbol{\xi}$.

Proof of Theorem 15.2. Choose $\beta:=\frac{1}{4 m}$, and let $B \subset[9 / 8,2]$ be the set of radii provided by Proposition 15.3. By a standard Fubini type argument analogous to what has been used in deriving (13.15) and the isoperimetric inequality $\bmod (p)$, we deduce that there exists $s \in B$ and an integer rectifiable current $R$ which is representative $\bmod (p)$ such that

$$
\partial R=\left\langle T-\mathbf{G}_{u}, \varphi, s\right\rangle \bmod (p) \text { and } \mathbf{M}(R) \leq C E^{\frac{2 m-1}{2 m-2}},
$$

where $u$ is the $E^{\beta}$-Lipschitz approximation of $T$ and $\varphi(x)=|x|$. Now, let $g$ be the Lipschitz map given in Proposition 15.3 corresponding with the choice $\sigma=s$. 
Since $\left.g\right|_{\partial B_{s}}=\left.u\right|_{\partial B_{s}}$, it also holds $\left\langle\mathbf{G}_{u}-\mathbf{G}_{g}, \varphi, s\right\rangle=0 \bmod (p)$. Furthermore, since $\left(\partial \mathbf{G}_{g}\right)\left\llcorner\mathbf{C}_{s}=0 \bmod (p)\right.$, and since $g$ takes values in $\Sigma$, the current $\mathbf{G}_{g}\left\llcorner\mathbf{C}_{s}+R\right.$ is a competitor for $T$ in $\mathbf{C}_{s}$, and thus, using [DLHMS, Equation (4.1)], the minimality of $T$ yields for some $\gamma>0$ :

$$
\begin{aligned}
\|T\|\left(\mathbf{C}_{s}\right) & \leq \| \mathbf{G}_{g}\left\llcorner\mathbf{C}_{s}+R \|\left(\mathbf{C}_{s}\right) \leq Q\left|B_{s}\right|+\frac{1}{2} \int_{B_{s}}|D g|^{2}+C E^{1+\gamma}\right. \\
& \stackrel{(15.9)}{\leq} Q\left|B_{s}\right|+\frac{1}{2} \int_{B_{s} \cap K}|D u|^{2}+C E^{\gamma}\left(E+\mathbf{A}^{2}\right)
\end{aligned}
$$

On the other hand, again by [DLHMS, Equation (4.1)] we also have:

$$
\begin{aligned}
\|T\|\left(\mathbf{C}_{s}\right) & =\|T\|\left(\left(B_{s} \backslash K\right) \times \mathbb{R}^{n}\right)+\left\|\mathbf{G}_{u}\right\|\left(\left(B_{s} \cap K\right) \times \mathbb{R}^{n}\right) \\
& \geq\|T\|\left(\left(B_{s} \backslash K\right) \times \mathbb{R}^{n}\right)+Q\left|B_{s} \cap K\right|+\frac{1}{2} \int_{B_{s} \cap K}|D u|^{2}-C E^{1+\gamma} .
\end{aligned}
$$

Combining (15.10) and (15.11) we conclude that $\mathbf{e}_{T}\left(B_{s} \backslash K\right) \leq C E^{\gamma}\left(E+\mathbf{A}^{2}\right)$. Now, we are able to prove the estimate (15.6). Let $A \subset B_{9 / 8}$ be any Borel set. We get:

$$
\begin{aligned}
\mathbf{e}_{T}(A)=\mathbf{e}_{T}(A \cap K)+\mathbf{e}_{T}(A \backslash K) & \leq \frac{1}{2} \int_{A \cap K}|D u|^{2}+C E^{1+\gamma}+\mathbf{e}_{T}\left(B_{s} \backslash K\right) \\
& \leq \frac{1}{2} \int_{A \cap K}|D u|^{2}+C E^{\gamma}\left(E+\mathbf{A}^{2}\right)
\end{aligned}
$$

On the other hand, observe that $|D u|(x)^{2} \leq C \mathbf{m}_{c} \mathbf{e}(x) \leq C E^{2 \beta}$ on $K$, and therefore $\mathbf{m}_{c} \mathbf{e}(x) \leq 1$ on $K$ if $E$ is suitably small. Let $q>0$ be the exponent given by Theorem 14.3, we deduce from (14.3) that

$$
\int_{A \cap K}|D u|^{2(1+q)} \leq C E^{1+q}
$$

and thus the Hölder inequality produces

$$
\int_{A \cap K}|D u|^{2} \leq\left(\int_{A \cap K}|D u|^{2(1+q)}\right)^{\frac{2}{1+q}}|A \cap K|^{\frac{q}{1+q}} \leq C E|A \cap K|^{\frac{q}{1+q}} .
$$

Plugging (15.13) into (15.12), we finally conclude (15.6), by possibly choosing a smaller $\gamma>0$.

As a corollary of Theorem 15.1 and of Theorem 13.3, we obtain the following result. 
Theorem 15.4. Let $\gamma$ be the constant of Theorem 15.1. Then, for every $\bar{\eta}>0$ there is a constant $\bar{\varepsilon}>0$ with the following property. Assume $T$ as in Assumption 9.1 is area minimizing $\bmod (p)$ in $\mathbf{C}_{4 r}(x), E=\mathbf{E}\left(T, \mathbf{C}_{4 r}(x)\right)<\bar{\varepsilon}$ and $r \mathbf{A} \leq \bar{\varepsilon} E^{1 / 2}$. If $u$ is the map in Theorem 15.1 and we fix good Cartesian coordinates, then there exists a Dir-minimizing $\bar{h}: B_{r}(x) \rightarrow \mathcal{A}_{Q}\left(\mathbb{R}^{\bar{n}}\right)$ if $Q<\frac{p}{2}$ or $\bar{h}: B_{r}(x) \rightarrow \mathscr{A}_{Q}\left(\mathbb{R}^{\bar{n}}\right)$ if $Q=\frac{p}{2}$ such that $h:=(\bar{h}, \Psi(\cdot, \bar{h}))$ satisfies

$$
r^{-2} \int_{B_{r}(x)} \mathcal{G}(u, h)^{2}+\int_{B_{r}(x)}(|D u|-|D h|)^{2}+\int_{B_{r}(x)}|D(\boldsymbol{\eta} \circ u)-D(\boldsymbol{\eta} \circ h)|^{2} \leq \bar{\eta} E r^{m} .
$$

\section{Strong approximation with the nonoriented excess}

In this section we show that it is possible to draw the same conclusions of the previous section replacing the cylindrical excess $\mathbf{E}\left(T, \mathbf{C}_{4 r}(x)\right)$ with the nonoriented $\mathbf{E}^{n o}\left(T, \mathbf{C}_{4 r}(x)\right)$ defined in (13.2). This will be vital, because in the remaining part of the paper we will in fact use mostly the nonoriented excess, which is structurally more suited to the arguments needed in the construction of the center manifold. As discussed in Remark 9.3, in the classical regularity theory for integral currents the cylindrical excess already possesses the required structural features; see [DLS14, Remark 2.5].

Theorem 16.1. There exist constants $\varepsilon, \gamma, C>0$ (depending on $m, \bar{n}, n, Q$ ) with the following property. Let $T$ be as in Assumption 9.1 in the cylinder $\mathbf{C}_{4 r}(x)$, and assume it is area minimizing $\bmod (p)$. Also assume that $E=\mathbf{E}\left(T, \mathbf{C}_{4 r}(x)\right)<\frac{1}{2}$ and that $E^{n o}:=\mathbf{E}^{n o}\left(T, \mathbf{C}_{4 r}(x)\right) \leq \varepsilon$. Then

$$
\mathbf{E}\left(T, \mathbf{C}_{2 r}(x)\right) \leq C \mathbf{E}^{n o}\left(T, \mathbf{C}_{4 r}(x)\right)+C \mathbf{A}^{2} r^{2},
$$

and in particular all the conclusions of Theorem 15.1 (and of Theorem 15.4, provided $r^{2} \mathbf{A}^{2} \leq \bar{\varepsilon}^{2} E \leq \bar{\varepsilon}^{3}$ for a suitable $\bar{\varepsilon}(\bar{\eta})>0$ ) hold in $B_{r}(x)$ with estimates where $E^{\text {no }}$ replaces $E$.

Before coming to the proof we state a simple variant of Theorem 15.1, where the estimates are inferred in a radius which is just slightly smaller than the starting one.

Proposition 16.2. There are a constant $C \geq 1$ and a $\bar{\varepsilon}>0$ with the following property. Let $\gamma$ be as in Theorem 15.1. Fix a cylinder $\mathbf{C}_{4 r}(x)$ and a current $T$ which satisfies all the assumptions of Theorem 15.1 with the stronger bound $E:=$ $\mathbf{E}\left(T, \mathbf{C}_{4 r}(x)\right) \leq \bar{\varepsilon}$. Choose $\omega$ such that $(1-\omega m)(1+\gamma)=1+\frac{\gamma}{2}$ and set $\rho=$ $r\left(1-C\left(E+r^{2} \mathbf{A}^{2}\right)^{\omega}\right)$. Then there are a map $u: B_{4 \rho}(x) \rightarrow \mathcal{A}_{Q}\left(\mathbb{R}^{n}\right)$ if $Q<\frac{p}{2}$, or $u: B_{4 \rho}(x) \rightarrow \mathscr{A}_{Q}\left(\mathbb{R}^{n}\right)$ if $Q=\frac{p}{2}$, and a closed set $K \subset B_{4 \rho}(x)$ such that:

$$
\operatorname{Gr}(u) \subset \Sigma,
$$




$$
\begin{gathered}
\operatorname{Lip}(u) \leq C\left(E+r^{2} \mathbf{A}^{2}\right)^{\gamma / 2} \quad \text { and } \quad \operatorname{osc}(u) \leq C \mathbf{h}\left(T, \mathbf{C}_{4 r}(x), \pi_{0}\right)+C r\left(E^{1 / 2}+r \mathbf{A}\right) \\
\mathbf{G}_{u}\left\llcorner\left(K \times \mathbb{R}^{n}\right)=T\left\llcorner\left(K \times \mathbb{R}^{n}\right) \bmod (p),\right.\right. \\
\left|B_{4 \rho}(x) \backslash K\right| \leq\|T\|\left(\left(B_{4 \rho}(x) \backslash K\right) \times \mathbb{R}^{n}\right) \leq C\left(E+r^{2} \mathbf{A}^{2}\right)^{1+\gamma / 2} r^{m} \\
\left.\left|\|T\|\left(\mathbf{C}_{4 \sigma \rho}(x)\right)-Q \omega_{m}(4 \sigma \rho)^{m}-\frac{1}{2} \int_{B_{4 \sigma \rho}(x)}\right| D u\right|^{2} \mid \leq\left(E+r^{2} \mathbf{A}^{2}\right)^{1+\gamma / 2} r^{m} \quad \forall 0<\sigma<1 .
\end{gathered}
$$

Proof. For every point $y \in B_{4 r\left(1-\left(E+r^{2} \mathbf{A}^{2}\right)^{\omega}\right)}(x)$ and a corresponding cylinder $\mathbf{C}^{y}:=$ $\mathbf{C}_{4 r\left(E+r^{2} \mathbf{A}^{2}\right)^{\omega}}(y)$, note that

$$
\mathbf{E}\left(T, \mathbf{C}^{y}\right)=\frac{\mathbf{e}_{T}\left(B_{4 r\left(E+r^{2} \mathbf{A}^{2}\right)^{\omega}}(y)\right)}{\omega_{m}(4 r)^{m}\left(E+r^{2} \mathbf{A}^{2}\right)^{m \omega}} \leq\left(E+r^{2} \mathbf{A}^{2}\right)^{-m \omega} \mathbf{E}\left(T, \mathbf{C}_{4 r}(x)\right) \leq E^{1-m \omega} .
$$

Thus, by choosing $\bar{\varepsilon}$ suitably small compared to $\varepsilon$ in Theorem 15.1 we fall under its assumptions. In particular, we find a function $u^{y}$ defined on the ball $B^{y}:=$ $B_{r\left(E+r^{2} \mathbf{A}^{2}\right)^{\omega}}(y)$ taking values into either $\mathcal{A}_{Q}\left(\mathbb{R}^{n}\right)$ or $\mathscr{A}_{Q}\left(\mathbb{R}^{n}\right)$ (depending on whether $Q<\frac{p}{2}$ or $Q=\frac{p}{2}$ ) and a set $K^{y}$ for which the following conclusions hold:

$$
\begin{gathered}
\operatorname{Gr}\left(u^{y}\right) \subset \Sigma, \\
\operatorname{Lip}\left(u^{y}\right) \leq C\left(E+\mathbf{A}^{2} r^{2}\right)^{(1-m \omega) \gamma}, \\
\mathbf{G}_{u^{y}}\left\llcorner\left(K^{y} \times \mathbb{R}^{n}\right)=T L\left(K^{y} \times \mathbb{R}^{n}\right) \quad \bmod (p),\right. \\
\left.\left|B^{y} \backslash K^{y}\right| \leq\|T\|\left(B^{y} \backslash K^{y}\right) \times \mathbb{R}^{n}\right) \leq C\left(E+r^{2} \mathbf{A}^{2}\right)^{(1-m \omega)(1+\gamma)}\left|B^{y}\right| .
\end{gathered}
$$

We now consider the regular lattice $\left(r\left(E+r^{2} \mathbf{A}^{2}\right)^{\omega}\right) /(\sqrt{m}) \mathbb{Z}^{m}$ and for each element $y$ of the lattice contained in $B_{4 r\left(1-\left(E+r^{2} \mathbf{A}^{2}\right)^{\omega}\right)}(x)$ we consider the corresponding ball $B^{y}$. Accordingly, we get a collection $\mathcal{B}$ of balls satisfying the following properties:

(o1) $\mathcal{B}$ covers $B_{4 \rho}(x)$.

(o2) The cardinality of $\mathcal{B}$ is bounded by $C\left(E+r^{2} \mathbf{A}^{2}\right)^{-m \omega}$ for a geometric constant $C=C(m)$.

(o3) Each element of $\mathcal{B}$ intersects at most $N$ elements of $\mathcal{B}$ for a geometric constant $N=N(m)$.

(o4) Every pair $z, w \in B_{4 \rho}(x)$ with $|z-w| \leq c(m) r\left(E+r^{2} \mathbf{A}^{2}\right)^{\omega}$ is contained in a single ball $B^{i}$, where $c(m)$ is a positive geometric constant.

(o5) For each pair $z, w \in B_{4 \rho}(x)$ with $\ell:=|z-w| \geq c(m) r\left(E+r^{2} \mathbf{A}^{2}\right)^{\omega}$ there is a chain of balls $B^{1}, \ldots, B^{\bar{N}} \in \mathcal{B}$ such that

(c1) $\bar{N} \leq C \ell r^{-1}\left(E+r^{2} \mathbf{A}^{2}\right)^{-\omega}$ for $C=C(m)$; 
(c2) $z \in B^{1}$ and $w \in B^{\bar{N}}$;

(c3) $\left|B^{i} \cap B^{i+1}\right| \geq \bar{c}(m) r^{m}\left(E+r^{2} \mathbf{A}^{2}\right)^{m \omega}$ for every $i=1, \ldots, \bar{N}-1$ for a geometric constant $\bar{c}(m)>0$.

We now consider for each $B^{i}=B^{y_{i}}$ the corresponding sets $\tilde{K}^{i}:=K^{y_{i}}$ and functions $u^{i}:=u^{y_{i}}$. We next define the sets

$$
K^{i}:=\tilde{K}^{i} \backslash \bigcup_{j: B^{j} \cap B^{i} \neq \emptyset}\left(B^{j} \backslash \tilde{K}^{j}\right) .
$$

We then set $K:=\bigcup_{i} K^{i}$ and observe that, by (o2), (o3) and (16.10), we must have

$$
\begin{aligned}
\left|B_{4 \rho}(x) \backslash K\right| & \leq\|T\|\left(\left(B_{4 \rho}(x) \backslash K\right) \times \mathbb{R}^{n}\right) \leq \sum_{i}\|T\|\left(\left(B^{i} \backslash K^{i}\right) \times \mathbb{R}^{n}\right) \\
& \leq C \rho^{m}\left(E+r^{2} \mathbf{A}^{2}\right)^{(1-m \omega)(1+\gamma)}=C \rho^{m}\left(E+r^{2} \mathbf{A}^{2}\right)^{1+\gamma / 2} .
\end{aligned}
$$

Next, we find a globally defined function $g$ on $K$ by setting $\left.g\right|_{K^{i}}:=\left.u^{i}\right|_{K^{i}}$. This function certainly enjoys the estimate $\operatorname{Lip}\left(\left.g\right|_{K^{i}}\right) \leq C\left(E+r^{2} \mathbf{A}^{2}\right)^{(1-m \omega) \gamma} \leq C(E+$ $\left.r^{2} \mathbf{A}^{2}\right)^{\gamma / 2}$ on each $K^{i}$. So, taken two points $z, w \in K$ with $|z-w| \leq c(m) r\left(E+r^{2} \mathbf{A}^{2}\right)^{\omega}$ we get, by (o4), the estimate

$$
\begin{aligned}
& \mathcal{G}(g(z), g(w)) \leq C\left(E+r^{2} \mathbf{A}^{2}\right)^{\gamma / 2}|z-w| \\
& \quad\left(\operatorname{resp} . \mathcal{G}_{s}(g(z), g(w)) \leq C\left(E+r^{2} \mathbf{A}^{2}\right)^{\gamma / 2}|z-w|\right) .
\end{aligned}
$$

If $\ell:=|z-w| \geq c(m) r\left(E+r^{2} \mathbf{A}^{2}\right)^{\omega}$, we use the chain of balls $B^{i}$ of (o5) and remark that, thanks to the estimate on $\left|B^{i} \backslash K^{i}\right|$, we can guarantee the existence of intermediate points $y_{i} \in K^{i} \cap K^{i+1}$ towards the estimate

$$
\begin{aligned}
& \mathcal{G}(g(z), g(w)) \leq C\left(E+r^{2} \mathbf{A}^{2}\right)^{\gamma / 2}|z-w| \\
& \quad\left(\operatorname{resp} . \mathcal{G}_{s}(g(z), g(w)) \leq C\left(E+r^{2} \mathbf{A}^{2}\right)^{\gamma / 2}|z-w|\right) .
\end{aligned}
$$

This proves that $g$ has the global Lipschitz bound $C\left(E+r^{2} \mathbf{A}^{2}\right)^{\gamma / 2}$ on $K$. Furthermore, since the graph $\mathbf{G}_{g}$ is $\bmod (p)$ equivalent to the current $T$ in the cylinder $K \times \mathbb{R}^{n}$, we have $\operatorname{osc}(g) \leq C \mathbf{h}\left(T, \mathbf{C}_{4 r}(x), \pi_{0}\right)$, see Remark 9.5. Now we can proceed as in Proposition 9.6 or Theorem 15.1. More precisely, we write $g=\sum_{i} \llbracket(h, \Psi(\cdot, h)) \rrbracket$, with $h: K \rightarrow \mathcal{A}_{Q}\left(\mathbb{R}^{\bar{n}}\right)$ if $Q<\frac{p}{2}$ or $h: K \rightarrow \mathscr{A}_{Q}\left(\mathbb{R}^{\bar{n}}\right)$ if $Q=\frac{p}{2}$. The map $h$ satisfies $\operatorname{Lip}(h) \leq C\left(E+r^{2} \mathbf{A}^{2}\right)^{\gamma / 2}$ and $\operatorname{osc}(h) \leq C \mathbf{h}\left(T, \mathbf{C}_{4 r}(x), \pi_{0}\right)$. Hence, taking advantage of [DLS11, Theorem 1.7] if $Q<\frac{p}{2}$ or [DLHMS, Corollary 5.3] when $Q=\frac{p}{2}$, we can extend $h$ to a map $\bar{h}: B_{4 \rho}(x) \rightarrow \mathcal{A}_{Q}\left(\mathbb{R}^{n}\right)\left(\right.$ resp. $\left.\bar{h}: B_{4 \rho}(x) \rightarrow \mathscr{A}_{Q}\left(\mathbb{R}^{n}\right)\right)$ which again satisfies $\operatorname{Lip}(\bar{h}) \leq C\left(E+r^{2} \mathbf{A}^{2}\right)^{\gamma / 2}$ and $\operatorname{osc}(\bar{h}) \leq C \mathbf{h}\left(T, \mathbf{C}_{4 r}(x), \pi_{0}\right)$. Finally, we set $u:=\sum_{i} \llbracket \bar{h}, \Psi(\cdot, \bar{h}) \rrbracket$, thus achieving

$$
\operatorname{Lip}(u) \leq C\left[\left(E+r^{2} \mathbf{A}^{2}\right)^{\frac{\gamma}{2}}+\|D \Psi\|_{0}\right], \quad \operatorname{osc}(u) \leq C \mathbf{h}\left(T, \mathbf{C}_{4 r}(x), \pi_{0}\right)+C r\|D \Psi\|_{0} .
$$


The estimate in (16.3) is then a consequence of the choice of coordinates discussed in Remark 13.1.

Finally, the estimate (16.6) is a consequence of the other ones, following the argument already given for (15.5). Since (16.2) and (16.4) are obvious by construction, this completes the proof.

Proof of Theorem 16.1. First of all we observe that it is enough to prove (16.1). Indeed, if $\varepsilon$ is sufficiently small, from (16.1) we conclude that we can apply Theorem 15.1 to any cylinder $\mathbf{C}_{4(r / 4)}(y)$ with $y \in B_{r}(x)$. Since $B_{r}(x)$ can be covered with a finite number $C(m)$ of balls $B_{r / 4}\left(y_{i}\right)$ with centers $y_{i} \in B_{r}(x)$, the existence of a suitable Lipschitz approximation over $B_{r}(x)$ follows easily. Theorem 15.4 can then be concluded by arguing as done for Theorem 13.3.

In order to show (16.1) we start observing that, by scaling and translating, we can assume $x=0$ and $r=1$. We then argue in several steps.

Step 1. First of all we claim that, for every $\delta>0$ there is $\varepsilon$ sufficiently small such that $\mathbf{E}\left(T, \mathbf{C}_{3}\right)<\delta$. Otherwise, by contradiction, there would be a sequence $\left\{T_{k}\right\}_{k=1}^{\infty}$ of area minimizing currents $\bmod (p)$ satisfying the hypotheses in Assumption 9.1 in $\mathbf{C}_{4}$ together with $\mathbf{E}\left(T_{k}, \mathbf{C}_{4}\right)<\frac{1}{2}$ for which $\mathbf{E}^{n o}\left(T_{k}, \mathbf{C}_{4}\right) \rightarrow 0$ and $\mathbf{M}^{p}\left(T_{k}\left\llcorner\mathbf{C}_{3}\right) \geq\right.$ $(Q+\delta) \omega_{m} 3^{m}$. In particular, because of the uniform bound on the excess, we can assume that $T_{k}$ converge, up to subsequences, to a $T$ which is an area minimizing current $\bmod (p)$ and satisfies Assumption 9.1. By convergence of the $\mathbf{M}^{p}$ in the interior, we also know that

$$
\mathbf{M}^{p}\left(T\left\llcorner\mathbf{C}_{3}\right) \geq(Q+\delta) \omega_{m} 3^{m}\right.
$$

On the other hand, since we can assume by Proposition 5.2 that $\mathbf{v}\left(T_{k}\left\llcorner\mathbf{C}_{4}\right) \rightarrow\right.$ $\mathbf{v}\left(T\left\llcorner\mathbf{C}_{4}\right)\right.$ as varifolds, and since the nonoriented excess is continuous in the varifold convergence, we must have $\mathbf{E}^{n o}\left(T, \mathbf{C}_{4}\right)=0$. Moreover, since $T$ is a representative $\bmod (p)$ we must have $\|T\|\left(\mathbf{C}_{4}\right) \leq \omega_{m}\left(Q+\frac{1}{2}\right) 4^{m}$ by the hypothesis that $\mathbf{E}\left(T_{k}, \mathbf{C}_{4}\right)<\frac{1}{2}$ for every $k$. The first condition implies that $T$ is supported in a finite number of planes parallel to $\pi_{0}$. By the constancy Lemma 7.4 we can assume that $T$ is a sum of integer multiples of $m$-dimensional disks of radius 4 parallel to $B_{4}\left(0, \pi_{0}\right)$. We thus have that the sum of the moduli of such integers must be at most $Q$. This contradicts (16.12).

Step 2. First of all, if $E:=\mathbf{E}\left(T, \mathbf{C}_{3}\right) \leq \mathbf{A}^{2}$, then there is nothing to prove. Hence, without loss of generality assume that

$$
E \geq \mathbf{A}^{2}
$$

Now apply Proposition 16.2 to obtain a Lipschitz map $u: B_{3-C E^{\omega}} \rightarrow \mathcal{A}_{Q}\left(\mathbb{R}^{n}\right)$ if $Q<\frac{p}{2}$ and $u: B_{3-C E^{\omega}} \rightarrow \mathscr{A}_{Q}\left(\mathbb{R}^{n}\right)$ if $Q=\frac{p}{2}$, and a closed set $K \subset B_{3-C E^{\omega}}(x)$ such that:

$$
\operatorname{Lip}(u) \leq C E^{\gamma / 2}
$$




$$
\begin{gathered}
\mathbf{G}_{u}\left\llcorner\left(K \times \mathbb{R}^{n}\right)=T\left\llcorner\left(K \times \mathbb{R}^{n}\right) \bmod (p),\right.\right. \\
\left|B_{3-C E^{\omega}} \backslash K\right| \leq C E^{1+\gamma / 2}, \\
\left.\left|\|T\|\left(\mathbf{C}_{3-C E^{\omega}}\right)-Q \omega_{m}\left(3-C E^{\omega}\right)^{m}-\frac{1}{2} \int_{B_{3-C E^{\omega}}}\right| D u\right|^{2} \mid \leq C E^{1+\gamma / 2} .
\end{gathered}
$$

Now we set $r_{1}:=3-C E^{\omega}, E_{1}:=\mathbf{E}\left(T, \mathbf{C}_{r_{1}}\right)$ and we consider the following three alternatives:

(a) $E_{1} \leq \mathbf{A}^{2}$

(b) $E_{1} \geq \max \left\{\frac{E}{2}, \mathbf{A}^{2}\right\}$;

(c) $\frac{E}{2} \geq E_{1} \geq \mathbf{A}^{2}$.

In the first case, assuming $\varepsilon$ sufficiently small, since $\mathbf{C}_{2} \subset \mathbf{C}_{r_{1}}$, we have concluded our desired estimate (16.1). In the second case observe first that from the estimates above we easily conclude

$$
\|T\|\left(\mathbf{C}_{r_{1}} \backslash\left(K \times \mathbb{R}^{n}\right)\right) \leq C E^{1+\gamma / 2} \leq C E_{1}^{1+\gamma / 2} .
$$

Consider now that, using $T\left\llcorner K \times \mathbb{R}^{n}=\mathbf{G}_{u}\left\llcorner K \times \mathbb{R}^{n}\right.\right.$ and standard computations, we have

$$
\|T\|\left(K \times \mathbb{R}^{n}\right)-Q|K|=\frac{1}{2} \int_{K \times \mathbb{R}^{n}}\left|\vec{T}(y)-\pi_{0}\right|_{n o}^{2} d\|T\| .
$$

We thus can combine these two estimates and claim

$$
E_{1}=\mathbf{E}\left(T, \mathbf{C}_{r_{1}}\right) \leq C E_{1}^{1+\gamma / 2}+\mathbf{E}^{n o}\left(T, \mathbf{C}_{r_{1}}\right) \leq \frac{E_{1}}{2}+C \mathbf{E}^{n o}\left(T, \mathbf{C}_{4}\right) .
$$

In particular we easily get

$$
\mathbf{E}\left(T, \mathbf{C}_{2}\right) \leq C \mathbf{E}\left(T, \mathbf{C}_{r_{1}}\right) \leq C \mathbf{E}^{n o}\left(T, \mathbf{C}_{4}\right),
$$

and again we have proved (16.1).

Finally, if we are in case (c) we iterate the step above and get a Lipschitz approximation in the cylinder $\mathbf{C}_{r_{2}}$ where $r_{2}=3-C E^{\omega}-C E_{1}^{\omega}$ and the new excess is $E_{2}:=\mathbf{E}\left(T, \mathbf{C}_{r_{2}}\right)$. We keep iterating this procedure which we stop at a certain radius

$$
r_{k}=3-C \sum_{i=0}^{k} E_{i}^{\omega}
$$

if either $E_{k} \leq \mathbf{A}^{2}$ or $E_{k} \geq \frac{E_{k-1}}{2}$. Observe that as long as the procedure does not end we have the recursive property $E_{i} \leq \frac{E_{i-1}}{2}$. We can thus estimate

$$
r_{k} \geq 3-C E^{\omega} \sum_{i=0}^{\infty} 2^{-\omega i} \geq 3-C \bar{C}(\omega) E^{\omega} .
$$


Since $\omega$ is a fixed exponent, provided $\delta>E$ is sufficiently small (which from the first step can be achieved by choosing $\varepsilon$ sufficiently small), we have $r_{k} \geq 2$. Thus, if the procedure stops we have proved (16.1). If the procedure does not stop, since $E_{k} \rightarrow 0$ we conclude easily that:

(i) $\mathbf{A}=0$;

(ii) if we set $r_{\infty}:=\lim _{k \rightarrow \infty} r_{k}$, then $2 \leq r_{\infty}$ and $\mathbf{E}\left(T, \mathbf{C}_{r_{\infty}}\right)=0$.

This implies that $\|T\|\left(\mathbf{C}_{r_{\infty}}\right)=Q \omega_{m} r_{\infty}^{m}$. Given that $\mathbf{p}_{\sharp} T\left\llcorner\mathbf{C}_{r_{\infty}}=Q \llbracket B_{r_{\infty}}\left(0, \pi_{0}\right) \rrbracket\right.$ $\bmod (p)$, this is only possible if the current $T$ in $\mathbf{C}_{r_{\infty}}$ consists of a finite number of disks parallel to $B_{r_{\infty}}\left(0, \pi_{0}\right)$ counted with integer multiplicities $\theta_{i}$ so that $\sum_{i}\left|\theta_{i}\right|=Q$. In particular, since $2 \leq r_{\infty}$, obviously $\mathbf{E}\left(T, \mathbf{C}_{2}\right)=0 \leq \mathbf{E}^{n o}\left(T, \mathbf{C}_{4}\right)$, which shows the validity of (16.1) even in this case.

\section{Part 3. Center manifold and approximation on its normal bundle}

This part of the paper deals with the construction of the center manifold. As it is the case with the proof of the partial regularity result for area minimizing currents in codimension higher than one, one might now attempt a proof of Theorems 4.3 and 8.2 carrying on the following program:

(1) Apply Almgren's strong approximation Theorem 15.1 to construct a sequence of Lipschitz maps $u_{k}$ approximating $T_{0, r_{k}}$ : here, $r_{k}$ is the contradiction sequence of radii appearing in Proposition 8.7, and the maps $u_{k}$ take values in $\mathcal{A}_{Q}\left(\pi_{0}^{\perp}\right)$ or in $\mathscr{A}_{Q}\left(\pi_{0}^{\perp}\right)$ depending on whether $Q<\frac{p}{2}$ or $Q=\frac{p}{2}$, respectively.

(2) Apply Theorem 15.4 to show that, after suitable normalization, a subsequence of the $u_{k}$ converges to a multiple valued map $u_{\infty}$ minimizing the Dirichlet energy (as in [DLS11] if $Q<\frac{p}{2}$ or as in [DLHMS] if $Q=\frac{p}{2}$ ).

(3) Use (iii) (resp. (iii)s) in Proposition 8.7 to infer that $u_{\infty}$ has a singular set of positive $\mathcal{H}^{m-2+\alpha}$ measure (resp. of positive $\mathcal{H}^{m-1+\alpha}$ measure), thus contradicting the linear theory in [DLS11] if $Q<\frac{p}{2}$ or in [DLHMS] if $Q=\frac{p}{2}$, respectively.

The obstacle towards the success of this program is making point (3) work, namely, showing that the "large" singular set of the currents persists in the limit as the approximating functions $u_{k}$ converge to $u_{\infty}$. As it was just stated, this is false: at this stage, nothing forces $u_{\infty}$ to actually exhibit any singularities. The center manifold construction is needed precisely to address this issue: when we approximate the current from the center manifold, we "subtract the regular part" of the Dirminimizer in the limit, which in turn allows us to close the contradiction argument.

In the first section of this part we will outline the arguments and present the statements of the main results. The subsequent sections will then be devoted to the proofs. 


\section{Outline and main results}

\subsection{Preliminaries for the construction of the center manifold.}

Notation 17.1 (Distance and nonoriented distance between m-planes). Throughout this part, $\pi_{0}$ continues to denote the plane $\mathbb{R}^{m} \times\{0\}$, with the standard orientation given by $\vec{\pi}_{0}=e_{1} \wedge \ldots \wedge e_{m}$. Given a $k$-dimensional plane $\pi$ in $\mathbb{R}^{m+n}$, we will in fact always identify $\pi$ with a simple unit $k$-vector $\vec{\pi}=v_{1} \wedge \ldots \wedge v_{k}$ orienting it (thereby making a distinction when the same plane is given opposite orientations). By a slight abuse of notation, given two $k$-planes $\pi_{1}$ and $\pi_{2}$, we will sometimes write $\left|\pi_{1}-\pi_{2}\right|$ in place of $\left|\vec{\pi}_{1}-\vec{\pi}_{2}\right|$, where the norm is induced by the standard inner product in $\Lambda_{k}\left(\mathbb{R}^{m+n}\right)$. Furthermore, for a given integer rectifiable current $T$, we recall the definition of $\left|\vec{T}(y)-\pi_{0}\right|_{n o}$ from (13.1). More in general, if $\pi_{1}$ and $\pi_{2}$ are two $k$-planes, we can define $\left|\pi_{1}-\pi_{2}\right|_{\text {no }}$ by

$$
\left|\pi_{1}-\pi_{2}\right|_{n o}:=\min \left\{\left|\vec{\pi}_{1}-\vec{\pi}_{2}\right|,\left|\vec{\pi}_{1}+\vec{\pi}_{2}\right|\right\} .
$$

It is understood that $\left|\pi_{1}-\pi_{2}\right|_{\text {no }}$ does not depend on the choice of the orientations $\vec{\pi}_{1}$ and $\vec{\pi}_{2}$.

Definition 17.2 (Excess and height). Given an integer rectifiable $m$-dimensional current $T$ which is a representative $\bmod (p)$ in $\mathbb{R}^{m+n}$ with finite mass and compact support and an $m$-plane $\pi$, we define the nonoriented excess of $T$ in the ball $\mathbf{B}_{r}(x)$ with respect to the plane $\pi$ as

$$
\mathbf{E}^{n o}\left(T, \mathbf{B}_{r}(x), \pi\right):=\left(2 \omega_{m} r^{m}\right)^{-1} \int_{\mathbf{B}_{r}(x)}|\vec{T}-\pi|_{n o}^{2} d\|T\| .
$$

The height function in a set $A \subset \mathbb{R}^{m+n}$ with respect to $\pi$ is

$$
\mathbf{h}(T, A, \pi):=\sup _{x, y \in \operatorname{spt}(T) \cap A}\left|\mathbf{p}_{\pi^{\perp}}(x)-\mathbf{p}_{\pi^{\perp}}(y)\right| .
$$

Definition 17.3 (Optimal planes). We say that an $m$-dimensional plane $\pi$ optimizes the nonoriented excess of $T$ in a ball $\mathbf{B}_{r}(x)$ if

$$
\mathbf{E}^{n o}\left(T, \mathbf{B}_{r}(x)\right):=\min _{\tau} \mathbf{E}^{n o}\left(T, \mathbf{B}_{r}(x), \tau\right)=\mathbf{E}^{n o}\left(T, \mathbf{B}_{r}(x), \pi\right)
$$

and if, in addition:

$$
\text { among all other } \pi^{\prime} \text { s.t. (17.2) holds, }\left|\pi-\pi_{0}\right| \text { is minimal. }
$$

Observe that in general the plane optimizing the nonoriented excess is not necessarily unique and $\mathbf{h}\left(T, \mathbf{B}_{r}(x), \pi\right)$ might depend on the optimizer $\pi$. Since for notational purposes it is convenient to define a unique "height" function $\mathbf{h}\left(T, \mathbf{B}_{r}(x)\right)$, we call a plane $\pi$ as in (17.2) and (17.3) optimal if in addition

$\mathbf{h}\left(T, \mathbf{B}_{r}(x)\right):=\min \left\{\mathbf{h}\left(T, \mathbf{B}_{r}(x), \tau\right): \tau\right.$ satisfies $(17.2)$ and $\left.(17.3)\right\}=\mathbf{h}\left(T, \mathbf{B}_{r}(x), \pi\right)$, 
i.e. $\pi$ optimizes the height among all planes that optimize the nonoriented excess. However (17.4) does not play any further role apart from simplifying the presentation.

REMark 17.4. Observe that there are two differences with [DLS16a, Definition 1.2]: first of all here we consider the nonoriented excess; secondly we have the additional requirement (17.3). In fact the point of (17.3) is to ensure that the planes $\pi$ "optimizing the nonoriented excess" always satisfy $\left|\pi-\pi_{0}\right|=\left|\pi-\pi_{0}\right|_{n o}$.

We are now ready to formulate the main assumptions of the statements in this section.

Assumption 17.5. $\left.\left.\varepsilon_{0} \in\right] 0,1\right]$ is a fixed constant and $\Sigma \subset \mathbf{B}_{7 \sqrt{m}} \subset \mathbb{R}^{m+n}$ is a $C^{3, \varepsilon_{0}}$ $(m+\bar{n})$-dimensional submanifold with no boundary in $\mathbf{B}_{7 \sqrt{m}}$. We moreover assume that, for each $q \in \Sigma, \Sigma$ is the graph of a $C^{3, \varepsilon_{0}}$ map $\Psi_{q}: T_{q} \Sigma \cap \mathbf{B}_{7 \sqrt{m}} \rightarrow T_{q} \Sigma^{\perp}$. We denote by $\mathbf{c}(\Sigma)$ the number $\sup _{q \in \Sigma}\left\|D \Psi_{q}\right\|_{C^{2, \varepsilon_{0}}} . T^{0}$ is an $m$-dimensional integer rectifiable current of $\mathbb{R}^{m+n}$ which is a representative $\bmod (p)$ and with support in $\Sigma \cap \overline{\mathbf{B}}_{6 \sqrt{m}} . T^{0}$ is area-minimizing $\bmod (p)$ in $\Sigma$ and moreover

$$
\begin{aligned}
\Theta\left(T^{0}, 0\right) & =Q \quad \text { and } \quad \partial T^{0}\left\llcorner\mathbf{B}_{6 \sqrt{m}}=0 \quad \bmod (p),\right. \\
\left\|T^{0}\right\|\left(\mathbf{B}_{6 \sqrt{m} \rho}\right) & \leq\left(\omega_{m} Q(6 \sqrt{m})^{m}+\varepsilon_{2}^{2}\right) \rho^{m} \quad \forall \rho \leq 1, \\
\mathbf{E}^{n o}\left(T^{0}, \mathbf{B}_{6 \sqrt{m}}\right) & =\mathbf{E}^{n o}\left(T^{0}, \mathbf{B}_{6 \sqrt{m}}, \pi_{0}\right), \\
\boldsymbol{m}_{0} & :=\max \left\{\mathbf{c}(\Sigma)^{2}, \mathbf{E}^{n o}\left(T^{0}, \mathbf{B}_{6 \sqrt{m}}\right)\right\} \leq \varepsilon_{2}^{2} \leq 1 .
\end{aligned}
$$

Here, $Q$ is a positive integer with $2 \leq Q \leq\left\lfloor\frac{p}{2}\right\rfloor$, and $\varepsilon_{2}$ is a positive number whose choice will be specified in each subsequent statement.

Constants depending only upon $m, n, \bar{n}$ and $Q$ will be called geometric and usually denoted by $C_{0}$.

Remark 17.6. Note that (17.8) implies $\mathbf{A}:=\left\|A_{\Sigma}\right\|_{C^{0}(\Sigma)} \leq C_{0} \boldsymbol{m}_{0}^{1 / 2}$, where $A_{\Sigma}$ denotes, as usual, the second fundamental form of $\Sigma$ and $C_{0}$ is a geometric constant. Observe further that for $q \in \Sigma$ the oscillation of $\Psi_{q}$ is controlled in $T_{q} \Sigma \cap \mathbf{B}_{6 \sqrt{m}}$ by $C_{0} \boldsymbol{m}_{0}^{1 / 2}$.

In what follows we set $l:=n-\bar{n}$. To avoid discussing domains of definitions it is convenient to extend $\Sigma$ so that it is an entire graph over all $T_{q} \Sigma$. Moreover we will often need to parametrize $\Sigma$ as the graph of a map $\Psi: \mathbb{R}^{m+\bar{n}} \rightarrow \mathbb{R}^{l}$. However we do not assume that $\mathbb{R}^{m+\bar{n}} \times\{0\}$ is tangent to $\Sigma$ at any $q$ and thus we need the following lemma. 
LEMMA 17.7. There are positive constants $C_{0}(m, \bar{n}, n)$ and $c_{0}(m, \bar{n}, n)$ such that, provided $\varepsilon_{2}<c_{0}$, the following holds. If $\Sigma$ is as in Assumption 17.5, then we can (modify it outside $\mathbf{B}_{6 \sqrt{m}}$ and) extend it to a complete submanifold of $\mathbb{R}^{m+n}$ which, for every $q \in \Sigma$, is the graph of a global $C^{3, \varepsilon_{0}}$ map $\Psi_{q}: T_{q} \Sigma \rightarrow T_{q} \Sigma^{\perp}$ with $\left\|D \Psi_{q}\right\|_{C^{2, \varepsilon_{0}}} \leq C_{0} \boldsymbol{m}_{0}^{1 / 2} . T^{0}$ is still area-minimizing $\bmod (p)$ in the extended manifold and in addition we can apply a global affine isometry which leaves $\mathbb{R}^{m} \times\{0\}$ fixed and maps $\Sigma$ onto $\Sigma^{\prime}$ so that

$$
\left|\mathbb{R}^{m+\bar{n}} \times\{0\}-T_{0} \Sigma^{\prime}\right| \leq C_{0} \boldsymbol{m}_{0}^{1 / 2}
$$

and $\Sigma^{\prime}$ is the graph of a $C^{3, \varepsilon_{0}}$ map $\Psi: \mathbb{R}^{m+\bar{n}} \rightarrow \mathbb{R}^{l}$ with $\Psi(0)=0$ and $\|D \Psi\|_{C^{2, \varepsilon_{0}}} \leq$ $C_{0} \boldsymbol{m}_{0}^{1 / 2}$.

From now on we assume w.l.o.g. that $\Sigma^{\prime}=\Sigma$. The next lemma is a standard consequence of the theory of area-minimizing currents (we include the proofs of Lemma 17.7 and Lemma 17.8 in Section 18 for the reader's convenience).

LEMMA 17.8. There are positive constants $C_{0}(m, n, \bar{n}, Q)$ and $c_{0}(m, n, \bar{n}, Q)$ with the following property. If $T^{0}$ is as in Assumption 17.5, $\varepsilon_{2}<c_{0}$ and $T:=T^{0}\left\llcorner\mathbf{B}_{23 \sqrt{m} / 4}\right.$, then:

$$
\begin{gathered}
\partial T\left\llcorner\mathbf{C}_{11 \sqrt{m} / 2}\left(0, \pi_{0}\right)=0 \bmod (p),\right. \\
\left(\mathbf{p}_{\pi_{0}}\right)_{\sharp} T\left\llcorner\mathbf{C}_{11 \sqrt{m} / 2}\left(0, \pi_{0}\right)=Q \llbracket B_{11 \sqrt{m} / 2}\left(0, \pi_{0}\right) \rrbracket \bmod (p),\right. \\
\text { and } \mathbf{h}\left(T, \mathbf{C}_{5 \sqrt{m}}\left(0, \pi_{0}\right)\right) \leq C_{0} \boldsymbol{m}_{0}^{1 / 2 m} .
\end{gathered}
$$

In particular, for each $x \in B_{11 \sqrt{m} / 2}\left(0, \pi_{0}\right)$ there is a point $q \in \operatorname{spt}(T)$ with $\mathbf{p}_{\pi_{0}}(q)=x$.

17.2 Construction of the center manifold. From now we will always work with the current $T$ of Lemma 17.8. We specify next some notation which will be recurrent in the paper when dealing with cubes of $\pi_{0}$. For each $j \in \mathbb{N}, \mathscr{C}^{j}$ denotes the family of closed cubes $L$ of $\pi_{0}$ of the form

$$
\left[a_{1}, a_{1}+2 \ell\right] \times \ldots \times\left[a_{m}, a_{m}+2 \ell\right] \times\{0\} \subset \pi_{0},
$$

where $2 \ell=2^{1-j}=: 2 \ell(L)$ is the side-length of the cube, $a_{i} \in 2^{1-j} \mathbb{Z} \forall i$ and we require in addition $-4 \leq a_{i} \leq a_{i}+2 \ell \leq 4$. To avoid cumbersome notation, we will usually drop the factor $\{0\}$ in (17.13) and treat each cube, its subsets and its points as subsets and elements of $\mathbb{R}^{m}$. Thus, for the center $x_{L}$ of $L$ we will use the notation $x_{L}=\left(a_{1}+\ell, \ldots, a_{m}+\ell\right)$, although the precise one is $\left(a_{1}+\ell, \ldots, a_{m}+\ell, 0, \ldots, 0\right)$. Next we set $\mathscr{C}:=\bigcup_{j \in \mathbb{N}} \mathscr{C}^{j}$. If $H$ and $L$ are two cubes in $\mathscr{C}$ with $H \subset L$, then we call $L$ an ancestor of $H$ and $H$ a descendant of $L$. When in addition $\ell(L)=2 \ell(H)$, $H$ is a son of $L$ and $L$ the father of $H$. 
Definition 17.9. A Whitney decomposition of $[-4,4]^{m} \subset \pi_{0}$ consists of a closed set $\Gamma \subset[-4,4]^{m}$ and a family $\mathscr{W} \subset \mathscr{C}$ satisfying the following properties:

(w1) $\Gamma \cup \bigcup_{L \in \mathscr{W}} L=[-4,4]^{m}$ and $\Gamma$ does not intersect any element of $\mathscr{W}$;

(w2) the interiors of any pair of distinct cubes $L_{1}, L_{2} \in \mathscr{W}$ are disjoint;

(w3) if $L_{1}, L_{2} \in \mathscr{W}$ have nonempty intersection, then $\frac{1}{2} \ell\left(L_{1}\right) \leq \ell\left(L_{2}\right) \leq 2 \ell\left(L_{1}\right)$.

Observe that (w1)-(w3) imply

$$
\operatorname{sep}(\Gamma, L):=\inf \{|x-y|: x \in L, y \in \Gamma\} \geq 2 \ell(L) \text { for every } L \in \mathscr{W} .
$$

However, we do not require any inequality of the form $\operatorname{sep}(\Gamma, L) \leq C \ell(L)$, although this would be customary for what is commonly called a Whitney decomposition in the literature.

The algorithm for the construction of the center manifold involves several parameters which depend in a complicated way upon several quantities and estimates. We introduce these parameters and specify some relations among them in the following

Assumption 17.10. $C_{e}, C_{h}, \beta_{2}, \delta_{2}, M_{0}$ are positive real numbers and $N_{0}$ is a natural number for which we assume always

$$
\beta_{2}=4 \delta_{2}=\min \left\{\frac{1}{2 m}, \frac{\gamma_{1}}{100}\right\},
$$

where $\gamma_{1}$ is the exponent in the estimates of Theorem 15.1,

$$
M_{0} \geq C_{0}(m, n, \bar{n}, Q) \geq 4 \quad \text { and } \quad \sqrt{m} M_{0} 2^{7-N_{0}} \leq 1 \text {. }
$$

As we can see, $\beta_{2}$ and $\delta_{2}$ are fixed. The other parameters are not fixed but are subject to further restrictions in the various statements, respecting the following "hierarchy". As already mentioned, "geometric constants" are assumed to depend only upon $m, n, \bar{n}$ and $Q$. The dependence of other constants upon the various parameters $p_{i}$ will be highlighted using the notation $C=C\left(p_{1}, p_{2}, \ldots\right)$.

Assumption 17.11. (Hierarchy of the parameters) In all the coming statements:

(a) $M_{0}$ is larger than a geometric constant (cf. (17.16)) or larger than a costant $C\left(\delta_{2}\right)$, see Proposition 17.29

(b) $N_{0}$ is larger than $C\left(\beta_{2}, \delta_{2}, M_{0}\right)$ (see for instance (17.16) and Proposition 17.32);

(c) $C_{e}$ is larger than $C\left(\beta_{2}, \delta_{2}, M_{0}, N_{0}\right)$ (see the statements of Proposition 17.13, Theorem 17.19 and Proposition 17.29);

(d) $C_{h}$ is larger than $C\left(\beta_{2}, \delta_{2}, M_{0}, N_{0}, C_{e}\right)$ (see Propositions 17.13 and 17.26 );

(e) $\varepsilon_{2}$ is smaller than $c\left(\beta_{2}, \delta_{2}, M_{0}, N_{0}, C_{e}, C_{h}\right)$ (which will always be positive).

The functions $C$ and $c$ will vary in the various statements: the hierarchy above guarantees however that there is a choice of the parameters for which all the restrictions required in the statements of the next propositions are simultaneously satisfied. To simplify our exposition, for smallness conditions on $\varepsilon_{2}$ as in (e) we will use the sentence " $\varepsilon_{2}$ is sufficiently small". 
Thanks to Lemma 17.8, for every $L \in \mathscr{C}$, we may choose $y_{L} \in \pi_{0}^{\perp}$ so that $p_{L}:=\left(x_{L}, y_{L}\right) \in \operatorname{spt}(T)$ (recall that $x_{L}$ is the center of $L$ ). $y_{L}$ is in general not unique and we fix an arbitrary choice. A more correct notation for $p_{L}$ would be $x_{L}+y_{L}$. This would however become rather cumbersome later, when we deal with various decompositions of the ambient space in triples of orthogonal planes. We thus abuse the notation slightly in using $(x, y)$ instead of $x+y$ and, consistently, $\pi_{0} \times \pi_{0}^{\perp}$ instead of $\pi_{0} \oplus \pi_{0}^{\perp}$.

Definition 17.12 (Refining procedure). For $L \in \mathscr{C}$ we set $r_{L}:=M_{0} \sqrt{m} \ell(L)$ and $\mathbf{B}_{L}:=\mathbf{B}_{64 r_{L}}\left(p_{L}\right)$. We next define the families of cubes $\mathscr{S} \subset \mathscr{C}$ and $\mathscr{W}=\mathscr{W}_{e} \cup \mathscr{W}_{h} \cup$ $\mathscr{W}_{n} \subset \mathscr{C}$ with the convention that $\mathscr{S}^{j}=\mathscr{S} \cap \mathscr{C}^{j}, \mathscr{W}^{j}=\mathscr{W} \cap \mathscr{C}^{j}$ and $\mathscr{W}_{\square}^{j}=\mathscr{W}_{\square} \cap \mathscr{C}^{j}$ for $\square=h, n, e$. We define $\mathscr{W}^{i}=\mathscr{S}^{i}=\emptyset$ for $i<N_{0}$. We proceed with $j \geq N_{0}$ inductively: if no ancestor of $L \in \mathscr{C}^{j}$ is in $\mathscr{W}$, then

(EX) $L \in \mathscr{W}_{e}^{j}$ if $\mathbf{E}^{n o}\left(T, \mathbf{B}_{L}\right)>C_{e} \boldsymbol{m}_{0} \ell(L)^{2-2 \delta_{2}}$;

(HT) $L \in \mathscr{W}_{h}^{j}$ if $L \notin \mathscr{W}_{e}^{j}$ and $\mathbf{h}\left(T, \mathbf{B}_{L}\right)>C_{h} \boldsymbol{m}_{0}^{1 / 2 m} \ell(L)^{1+\beta_{2}}$;

(NN) $L \in \mathscr{W}_{n}^{j}$ if $L \notin \mathscr{W}_{e}^{j} \cup \mathscr{W}_{h}^{j}$ but it intersects an element of $\mathscr{W}^{j-1}$;

if none of the above occurs, then $L \in \mathscr{S}^{j}$. We finally set

$$
\Gamma:=[-4,4]^{m} \backslash \bigcup_{L \in \mathscr{W}} L=\bigcap_{j \geq N_{0}} \bigcup_{L \in \mathscr{S}^{j}} L .
$$

Observe that, if $j>N_{0}$ and $L \in \mathscr{S}^{j} \cup \mathscr{W}^{j}$, then necessarily its father belongs to $\mathscr{S}^{j-1}$.

Proposition 17.13 (Whitney decomposition). Let Assumptions 17.5 and 17.10 hold and let $\varepsilon_{2}$ be sufficiently small. Then $(\Gamma, \mathscr{W})$ is a Whitney decomposition of $[-4,4]^{m} \subset \pi_{0}$. Moreover, for any choice of $M_{0}$ and $N_{0}$, there is $C^{\star}:=C^{\star}\left(M_{0}, N_{0}\right)$ such that, if $C_{e} \geq C^{\star}$ and $C_{h} \geq C^{\star} C_{e}$, then

$$
\mathscr{W}^{j}=\emptyset \quad \text { for all } j \leq N_{0}+6 .
$$

Finally, the following estimates hold with $C=C\left(\beta_{2}, \delta_{2}, M_{0}, N_{0}, C_{e}, C_{h}\right)$ :

$$
\begin{aligned}
& \mathbf{E}^{n o}\left(T, \mathbf{B}_{J}\right) \leq C_{e} \boldsymbol{m}_{0} \ell(J)^{2-2 \delta_{2}} \quad \text { and } \quad \mathbf{h}\left(T, \mathbf{B}_{J}\right) \leq C_{h} \boldsymbol{m}_{0}^{1 / 2 m} \ell(J)^{1+\beta_{2}} \quad \forall J \in \mathscr{S}, \\
& \mathbf{E}^{n o}\left(T, \mathbf{B}_{L}\right) \leq C \boldsymbol{m}_{0} \ell(L)^{2-2 \delta_{2}} \quad \text { and } \quad \mathbf{h}\left(T, \mathbf{B}_{L}\right) \leq C \boldsymbol{m}_{0}^{1 / 2 m} \ell(L)^{1+\beta_{2}} \quad \forall L \in \mathscr{W} .
\end{aligned}
$$

We will prove Proposition 17.13 in Section 19. Next, we fix two important functions $\vartheta, \varrho: \mathbb{R}^{m} \rightarrow \mathbb{R}$

Assumption 17.14. $\varrho \in C_{c}^{\infty}\left(B_{1}\right)$ is radial, $\int \varrho=1$ and $\int|x|^{2} \varrho(x) d x=0$. For $\lambda>0 \varrho_{\lambda}$ denotes, as usual, $x \mapsto \lambda^{-m} \varrho\left(\frac{x}{\lambda}\right) . \vartheta \in C_{c}^{\infty}\left(\left[-\frac{17}{16}, \frac{17}{16}\right]^{m},[0,1]\right)$ is identically 1 on $[-1,1]^{m}$. 
$\varrho$ will be used as convolution kernel for smoothing maps $z$ defined on $m$-dimensional planes $\pi$ of $\mathbb{R}^{m+n}$. In particular, having fixed an isometry $A$ of $\mathbb{R}^{m}$ onto $\pi$, the smoothing will be given by $\left[(z \circ A) * \varrho_{\lambda}\right] \circ A^{-1}$. Observe that since $\varrho$ is radial, our map does not depend on the choice of the isometry and we will therefore use the shorthand notation $z * \varrho_{\lambda}$.

Definition 17.15 ( $\pi$-approximations). Let $L \in \mathscr{S} \cup \mathscr{W}$ and $\pi$ be an $m$-dimensional plane. If $T\left\llcorner\mathbf{C}_{32 r_{L}}\left(p_{L}, \pi\right)\right.$ fulfills the assumptions of Theorem 16.1 in the cylinder $\mathbf{C}_{32 r_{L}}\left(p_{L}, \pi\right)$, then the resulting map $u$ given by the theorem, which is defined on $B_{8 r_{L}}\left(p_{L}, \pi\right)$ and takes values either in $\mathcal{A}_{Q}\left(\pi^{\perp}\right)$ (if $Q<\frac{p}{2}$ ) or in $\mathscr{A}_{Q}\left(\pi^{\perp}\right.$ ) (if $Q=\frac{p}{2}$ ) is called a $\pi$-approximation of $T$ in $\mathbf{C}_{8 r_{L}}\left(p_{L}, \pi\right)$. The map $\hat{h}: B_{7 r_{L}}\left(p_{L}, \pi\right) \rightarrow \pi^{\perp}$ given by $\hat{h}:=(\boldsymbol{\eta} \circ u) * \varrho_{\ell(L)}$ will be called the smoothed average of the $\pi$-approximation.

Definition 17.16 (Reference plane $\pi_{L}$ ). For each $L \in \mathscr{S} \cup \mathscr{W}$ we let $\hat{\pi}_{L}$ be an optimal plane in $\mathbf{B}_{L}$ (cf. Definition 17.3) and choose an m-plane $\pi_{L} \subset T_{p_{L}} \Sigma$ which minimizes $\left|\hat{\pi}_{L}-\pi_{L}\right|$.

The following lemma, which will be proved in Section 19, deals with graphs of multivalued functions $f$ in several systems of coordinates.

Lemma 17.17. Let the assumptions of Proposition 17.13 hold and assume $C_{e} \geq$ $C^{\star}$ and $C_{h} \geq C^{\star} C_{e}$ (where $C^{\star}$ is the constant of Proposition 17.13). For any choice of the other parameters, if $\varepsilon_{2}$ is sufficiently small, then $T\left\llcorner\mathbf{C}_{32 r_{L}}\left(p_{L}, \pi_{L}\right)\right.$ satisfies the assumptions of Theorem 16.1 for any $L \in \mathscr{W} \cup \mathscr{S}$. Moreover, if $f_{L}$ is a $\pi_{L}$-approximation, denote by $\hat{h}_{L}$ its smoothed average and by $\bar{h}_{L}$ the map $\mathbf{p}_{T_{p_{L}} \Sigma}\left(\hat{h}_{L}\right)$, which takes values in the plane $\varkappa_{L}:=T_{p_{L}} \Sigma \cap \pi_{L}^{\perp}$, i.e. the orthogonal complement of $\pi_{L}$ in $T_{p_{L}} \Sigma$. If we let $h_{L}$ be the map $x \in B_{7 r_{L}}\left(p_{L}, \pi_{L}\right) \mapsto$ $h_{L}(x):=\left(\bar{h}_{L}(x), \Psi_{p_{L}}\left(x, \bar{h}_{L}(x)\right)\right) \in \varkappa_{L} \times T_{p_{L}} \Sigma^{\perp}$, then there is a smooth map $g_{L}$ : $B_{4 r_{L}}\left(p_{L}, \pi_{0}\right) \rightarrow \pi_{0}^{\perp}$ such that $\mathbf{G}_{g_{L}}=\mathbf{G}_{h_{L}}\left\llcorner\mathbf{C}_{4 r_{L}}\left(p_{L}, \pi_{0}\right)\right.$.

For the sake of simplicity, in the future we will sometimes regard $g_{L}$ as a map $g_{L}: B_{4 r_{L}}\left(x_{L}, \pi_{0}\right) \rightarrow \pi_{0}^{\perp}$ rather than as a map $g_{L}: B_{4 r_{L}}\left(p_{L}, \pi_{0}\right) \rightarrow \pi_{0}^{\perp}$. In particular, we will sometimes consider $g_{L}(x)$ with $x \in B_{4 r_{L}}\left(x_{L}, \pi_{0}\right)$ even though the correct writing is the more cumbersome $g_{L}\left(\left(x, y_{L}\right)\right)$.

Definition 17.18 (Interpolating functions). The maps $h_{L}$ and $g_{L}$ in Lemma 17.17 will be called, respectively, the tilted $L$-interpolating function and the $L$-interpolating function. For each $j$ let $\mathscr{P}^{j}:=\mathscr{S}^{j} \cup \bigcup_{i=N_{0}}^{j} \mathscr{W}^{i}$ and for $L \in \mathscr{P}^{j}$ define $\vartheta_{L}(y):=$ $\vartheta\left(\frac{y-x_{L}}{\ell(L)}\right)$. Set

$$
\left.\hat{\varphi}_{j}:=\frac{\sum_{L \in \mathscr{P}^{j}} \vartheta_{L} g_{L}}{\sum_{L \in \mathscr{P}^{j}} \vartheta_{L}} \quad \text { on }\right]-4,4\left[^{m},\right.
$$

let $\bar{\varphi}_{j}(y)$ be the first $\bar{n}$ components of $\hat{\varphi}_{j}(y)$ and define $\varphi_{j}(y):=\left(\bar{\varphi}_{j}(y), \Psi\left(y, \bar{\varphi}_{j}(y)\right)\right)$, where $\Psi$ is the map of Lemma 17.7. $\varphi_{j}$ will be called the glued interpolation at the step $j$. 
Theorem 17.19 (Existence of the center manifold). Assume that the hypotheses of the Lemma 17.17 hold and let $\kappa:=\min \left\{\varepsilon_{0} / 2, \beta_{2} / 4\right\}$. For any choice of the other parameters, if $\varepsilon_{2}$ is sufficiently small, then

(i) $\left\|D \varphi_{j}\right\|_{C^{2, \kappa}} \leq C \boldsymbol{m}_{0}^{1 / 2}$ and $\left\|\varphi_{j}\right\|_{C^{0}} \leq C \boldsymbol{m}_{0}^{1 / 2 m}$, with $C=C\left(\beta_{2}, \delta_{2}, M_{0}, N_{0}, C_{e}, C_{h}\right)$;

(ii) if $L \in \mathscr{W}^{i}$ and $H$ is a cube concentric to $L$ with $\ell(H)=\frac{9}{8} \ell(L)$, then $\varphi_{j}=\varphi_{k}$ on $H$ for any $j, k \geq i+2$;

(iii) $\varphi_{j}$ converges in $C^{3}$ to a map $\varphi$ and $\mathcal{M}:=\operatorname{Gr}\left(\left.\varphi\right|_{]-4,4[m}\right)$ is a $C^{3, \kappa}$ submanifold of $\Sigma$.

Definition 17.20 (Whitney regions). The manifold $\mathcal{M}$ in Theorem 17.19 is called a center manifold of $T$ relative to $\pi_{0}$, and $(\Gamma, \mathscr{W})$ the Whitney decomposition associated to $\mathcal{M}$. Setting $\Phi(y):=(y, \varphi(y))$, we call $\Phi(\Gamma)$ the contact set. Moreover, to each $L \in \mathscr{W}$ we associate a Whitney region $\mathcal{L}$ on $\mathcal{M}$ as follows:

(WR) $\mathcal{L}:=\Phi\left(H \cap\left[-\frac{7}{2}, \frac{7}{2}\right]^{m}\right)$, where $H$ is the cube concentric to $L$ with $\ell(H)=\frac{17}{16} \ell(L)$.

We will present a proof of Theorem 17.19 in Section 20

17.3 The $\mathcal{M}$-normal approximation and related estimates. In what follows we assume that the conclusions of Theorem 17.19 apply and denote by $\mathcal{M}$ the corresponding center manifold. For any Borel set $\mathcal{V} \subset \mathcal{M}$ we will denote by $|\mathcal{V}|$ its $\mathcal{H}^{m}$-measure and will write $\int_{\mathcal{V}} f$ for the integral of $f$ with respect to $\mathcal{H}^{m}\left\llcorner\mathcal{V} . \mathcal{B}_{r}(q)\right.$ denotes the geodesic open balls in $\mathcal{M}$.

Assumption 17.21. We fix the following notation and assumptions.

(U) $\mathbf{U}:=\left\{x \in \mathbb{R}^{m+n}: \exists ! y=\mathbf{p}(x) \in \mathcal{M}\right.$ with $|x-y|<1$ and $\left.(x-y) \perp \mathcal{M}\right\}$.

(P) $\mathbf{p}: \mathbf{U} \rightarrow \mathcal{M}$ is the map defined by (U).

(R) For any choice of the other parameters, we assume $\varepsilon_{2}$ to be so small that $\mathbf{p}$ extends to $C^{2, \kappa}(\overline{\mathbf{U}})$ and $\mathbf{p}^{-1}(y)=y+\overline{B_{1}\left(0,\left(T_{y} \mathcal{M}\right)^{\perp}\right)}$ for every $y \in \mathcal{M}$.

(L) We denote by $\partial_{l} \mathbf{U}:=\mathbf{p}^{-1}(\partial \mathcal{M})$ the lateral boundary of $\mathbf{U}$.

The following is then a corollary of Theorem 17.19 and the construction algorithm; see Section 21 for the proof.

Corollary 17.22. Under the hypotheses of Theorem 17.19 and of Assumption 17.21 we have:

(i) $\operatorname{spt}^{p}\left(\partial(T\llcorner\mathbf{U})) \subset \partial_{l} \mathbf{U}, \operatorname{spt}\left(T\left\llcorner\left[-\frac{7}{2}, \frac{7}{2}\right]^{m} \times \mathbb{R}^{n}\right) \subset \mathbf{U}\right.\right.$, and $\mathbf{p}_{\sharp}(T\llcorner\mathbf{U})=Q \llbracket \mathcal{M} \rrbracket$ $\bmod (p)$;

(ii) $\operatorname{spt}(\langle T, \mathbf{p}, \Phi(q)\rangle) \subset\left\{y:|\Phi(q)-y| \leq C \boldsymbol{m}_{0}^{1 / 2 m} \ell(L)^{1+\beta_{2}}\right\}$ for every $q \in L \in \mathscr{W}$, where

$C=C\left(\beta_{2}, \delta_{2}, M_{0}, N_{0}, C_{e}, C_{h}\right)$;

(iii) $\langle T, \mathbf{p}, q\rangle=Q \llbracket q \rrbracket$ for every $q \in \Phi(\Gamma)$.

The next main goal is to couple the center manifold of Theorem 17.19 with a good approximating map defined on it. 
Definition 17.23 ( $\mathcal{M}$-normal approximation). An $\mathcal{M}$-normal approximation of $T$ is given by a pair $(\mathcal{K}, F)$ with the following properties. $\mathcal{K} \subset \mathcal{M}$ is closed and contains $\Phi\left(\Gamma \cap\left[-\frac{7}{2}, \frac{7}{2}\right]^{m}\right)$. Moreover:

(a) If $Q=\frac{p}{2}, F$ is a Lipschitz map which takes values in $\mathscr{A}_{Q}\left(\mathbb{R}^{m+n}\right)$ and satisfies the requirements of [DLHMS, Assumption 11.1].

(b) If $Q<\frac{p}{2}, F$ is a Lipschitz map which takes values in $\mathcal{A}_{Q}\left(\mathbb{R}^{m+n}\right)$ and has the special form $F(x)=\sum_{i} \llbracket x+N_{i}(x) \rrbracket$.

In both cases we require that

(A1) $\operatorname{spt}\left(\mathbf{T}_{F}\right) \subset \Sigma$;

(A2) $\mathbf{T}_{F}\left\llcorner\mathbf{p}^{-1}(\mathcal{K})=T\left\llcorner\mathbf{p}^{-1}(\mathcal{K}) \bmod (p)\right.\right.$,

where $\mathbf{T}_{F}$ is the integer rectifiable current induced by $F$; see [DLHMS, Definition 11.2]. The map $N$ (for the case $Q=\frac{p}{2}$ see [DLHMS, Assumption 11.1]) is the normal part of $F$.

In the definition above it is not required that the map $F$ approximates efficiently the current outside the set $\Phi\left(\Gamma \cap\left[-\frac{7}{2}, \frac{7}{2}\right]^{m}\right)$. However, all the maps constructed will approximate $T$ with a high degree of accuracy in each Whitney region: such estimates are detailed in the next theorem, the proof of which will be tackled in Section 21.

Theorem 17.24 (Local estimates for the $\mathcal{M}$-normal approximation). Let $\gamma_{2}:=\frac{\gamma}{4}$, with $\gamma$ the constant of Theorem 15.1. Under the hypotheses of Theorem 17.19 and Assumption 17.21, if $\varepsilon_{2}$ is suitably small (depending upon all other parameters), then there is an $\mathcal{M}$-normal approximation $(\mathcal{K}, F)$ such that the following estimates hold on every Whitney region $\mathcal{L}$ associated to a cube $L \in \mathscr{W}$, with constants $C=$ $C\left(\beta_{2}, \delta_{2}, M_{0}, N_{0}, C_{e}, C_{h}\right)$ :

$$
\begin{gathered}
\operatorname{Lip}\left(\left.N\right|_{\mathcal{L}}\right) \leq C \boldsymbol{m}_{0}^{\gamma_{2}} \ell(L)^{\gamma_{2}} \quad \text { and } \quad\left\|\left.N\right|_{\mathcal{L}}\right\|_{C^{0}} \leq C \boldsymbol{m}_{0}^{1 / 2 m} \ell(L)^{1+\beta_{2}}, \\
|\mathcal{L} \backslash \mathcal{K}|+\left\|\mathbf{T}_{F}-T\right\|_{p}\left(\mathbf{p}^{-1}(\mathcal{L})\right) \leq C \boldsymbol{m}_{0}^{1+\gamma_{2}} \ell(L)^{m+2+\gamma_{2}} \\
\int_{\mathcal{L}}|D N|^{2} \leq C \boldsymbol{m}_{0} \ell(L)^{m+2-2 \delta_{2}}
\end{gathered}
$$

Moreover, for any $a>0$ and any Borel $\mathcal{V} \subset \mathcal{L}$, we have $\left(\right.$ for $C=C\left(\beta_{2}, \delta_{2}, M_{0}, N_{0}\right.$, $\left.C_{e}, C_{h}\right)$ )

$$
\int_{\mathcal{V}}|\boldsymbol{\eta} \circ N| \leq C \boldsymbol{m}_{0}\left(\ell(L)^{m+3+\beta_{2} / 3}+a \ell(L)^{2+\gamma_{2} / 2}|\mathcal{V}|\right)+\frac{C}{a} \int_{\mathcal{V}} \mathcal{G}_{\square}(N, Q \llbracket \boldsymbol{\eta} \circ N \rrbracket)^{2+\gamma_{2}},
$$

where $\square=s$ in case $p=2 Q$, and it is empty otherwise.

From (17.22) to (17.24) it is not difficult to infer analogous "global versions" of the estimates. 
Corollary 17.25 (Global estimates). Let $\mathcal{M}^{\prime}$ be the domain $\Phi\left(\left[-\frac{7}{2}, \frac{7}{2}\right]^{m}\right)$ and $N$ the map of Theorem 17.24. Then, (again with $C=C\left(\beta_{2}, \delta_{2}, M_{0}, N_{0}, C_{e}, C_{h}\right)$ )

$$
\begin{gathered}
\operatorname{Lip}\left(\left.N\right|_{\mathcal{M}^{\prime}}\right) \leq C \boldsymbol{m}_{0}^{\gamma_{2}} \text { and }\left\|\left.N\right|_{\mathcal{M}^{\prime}}\right\|_{C^{0}} \leq C \boldsymbol{m}_{0}^{1 / 2 m} \\
\left|\mathcal{M}^{\prime} \backslash \mathcal{K}\right|+\left\|\mathbf{T}_{F}-T\right\|_{p}\left(\mathbf{p}^{-1}\left(\mathcal{M}^{\prime}\right)\right) \leq C \boldsymbol{m}_{0}^{1+\gamma_{2}} \\
\int_{\mathcal{M}^{\prime}}|D N|^{2} \leq C \boldsymbol{m}_{0} .
\end{gathered}
$$

17.4 Separation and domains of influence of large excess cubes. We now analyze more in detail the consequences of the various stopping conditions for the cubes in $\mathscr{W}$. We first deal with $L \in \mathscr{W}_{h}$.

Proposition 17.26 (Separation). There is a constant $C^{\sharp}\left(M_{0}\right)>0$ with the following property. Assume the hypotheses of Theorem 17.24 and in addition $C_{h}^{2 m} \geq C^{\sharp} C_{e}$. If $\varepsilon_{2}$ is sufficiently small, then the following conclusions hold for every $L \in \mathscr{W}_{h}$ :

(S1) $\Theta(T, q) \leq Q-\frac{1}{2}$ for every $q \in \mathbf{B}_{16 r_{L}}\left(p_{L}\right)$;

(S2) $L \cap H=\emptyset$ for every $H \in \mathscr{W}_{n}$ with $\ell(H) \leq \frac{1}{2} \ell(L)$;

$(\mathrm{S} 3) \mathcal{G}_{\square}(N(x), Q \llbracket \boldsymbol{\eta} \circ N(x) \rrbracket) \geq \frac{1}{4} C_{h} \boldsymbol{m}_{0}^{1 / 2 m} \ell(L)^{1+\beta_{2}}$ for every $x \in \Phi\left(B_{2 \sqrt{m} \ell(L)}\left(x_{L}, \pi_{0}\right)\right)$, where $\square=s$ if $p=2 Q$ or $\square=$ otherwise.

A simple corollary of the previous proposition is the following.

Corollary 17.27 Given any $H \in \mathscr{W}_{n}$ there is a chain $L=L_{0}, L_{1}, \ldots, L_{j}=H$ such that:

(a) $L_{0} \in \mathscr{W}_{e}$ and $L_{i} \in \mathscr{W}_{n}$ for all $i>0$;

(b) $L_{i} \cap L_{i-1} \neq \emptyset$ and $\ell\left(L_{i}\right)=\frac{1}{2} \ell\left(L_{i-1}\right)$ for all $i>0$.

In particular, $H \subset B_{3 \sqrt{m} \ell(L)}\left(x_{L}, \pi_{0}\right)$.

We use this last corollary to partition $\mathscr{W}_{n}$.

Definition 17.28 (Domains of influence). We first fix an ordering of the cubes in $\mathscr{W}_{e}$ as $\left\{J_{i}\right\}_{i \in \mathbb{N}}$ so that their sidelengths do not increase. Then $H \in \mathscr{W}_{n}$ belongs to $\mathscr{W}_{n}\left(J_{0}\right)$ (the domain of influence of $J_{0}$ ) if there is a chain as in Corollary 17.27 with $L_{0}=J_{0}$. Inductively, $\mathscr{W}_{n}\left(J_{r}\right)$ is the set of cubes $H \in \mathscr{W}_{n} \backslash \cup_{i<r} \mathscr{W}_{n}\left(J_{i}\right)$ for which there is a chain as in Corollary 17.27 with $L_{0}=J_{r}$.

17.5 Splitting before tilting. The following proposition contains a "typical" splitting-before-tilting phenomenon: the key assumption of the theorem (i.e. $L \in$ $\mathscr{W}_{e}$ ) is that the excess does not decay at some given scale ("tilting") and the main conclusion (17.30) implies a certain amount of separation between the sheets of the current ("splitting"); see Section 22 for the proof. 
Proposition 17.29 (Splitting I). There are functions $C_{1}\left(\delta_{2}\right), C_{2}\left(M_{0}, \delta_{2}\right)$ such that, if $M_{0} \geq C_{1}\left(\delta_{2}\right), C_{e} \geq C_{2}\left(M_{0}, \delta_{2}\right)$, if the hypotheses of Theorem 17.24 hold and if $\varepsilon_{2}$ is chosen sufficiently small, then the following holds. If $L \in \mathscr{W}_{e}, q \in \pi_{0}$ with $\operatorname{dist}(L, q) \leq$ $4 \sqrt{m} \ell(L)$ and $\Omega=\Phi\left(B_{\ell(L) / 4}\left(q, \pi_{0}\right)\right)$, then (with $C, C_{3}=C\left(\beta_{2}, \delta_{2}, M_{0}, N_{0}, C_{e}, C_{h}\right)$ ):

$$
\begin{gathered}
C_{e} \boldsymbol{m}_{0} \ell(L)^{m+2-2 \delta_{2}} \leq \ell(L)^{m} \mathbf{E}^{n o}\left(T, \mathbf{B}_{L}\right) \leq C \int_{\Omega}|D N|^{2}, \\
\int_{\mathcal{L}}|D N|^{2} \leq C \ell(L)^{m} \mathbf{E}^{n o}\left(T, \mathbf{B}_{L}\right) \leq C_{3} \ell(L)^{-2} \int_{\Omega}|N|^{2} .
\end{gathered}
$$

17.6 Persistence of multiplicity $\boldsymbol{Q}$ points. We next state two important properties triggered by the existence of $q \in \operatorname{spt}(T)$ with $\Theta(T, q)=Q$, both related to the splitting before tilting. Their proofs will be discussed in Section 23.

Proposition 17.30 (Splitting II). Let the hypotheses of Theorem 17.19 hold and assume $\varepsilon_{2}$ is sufficiently small. For any $\alpha, \bar{\alpha}, \hat{\alpha}>0$, there is $\varepsilon_{3}=\varepsilon_{3}\left(\alpha, \bar{\alpha}, \hat{\alpha}, \beta_{2}, \delta_{2}\right.$, $\left.M_{0}, N_{0}, C_{e}, C_{h}\right)>0$ as follows.

When $Q<\frac{p}{2}$, if for some $s \leq 1$

$$
\begin{aligned}
& \sup \left\{\ell(L): L \in \mathscr{W}, L \cap B_{3 s}\left(0, \pi_{0}\right) \neq \emptyset\right\} \leq s, \\
& \mathcal{H}_{\infty}^{m-2+\alpha}\left(\{\Theta(T, \cdot)=Q\} \cap \mathbf{B}_{s}\right) \geq \bar{\alpha} s^{m-2+\alpha},
\end{aligned}
$$

and $\min \left\{s, \boldsymbol{m}_{0}\right\} \leq \varepsilon_{3}$, then,

$$
\sup \left\{\ell(L): L \in \mathscr{W}_{e} \text { and } L \cap B_{19 s / 16}\left(0, \pi_{0}\right) \neq \emptyset\right\} \leq \hat{\alpha} s .
$$

When $Q=\frac{p}{2}$, the same conclusion can be reached if (17.32) is replaced by

$$
\mathcal{H}_{\infty}^{m-1+\alpha}\left(\{\Theta(T, \cdot)=Q\} \cap \mathbf{B}_{s}\right) \geq \bar{\alpha} s^{m-1+\alpha} .
$$

Proposition 17.31 (Persistence of $Q$-points). Assume the hypotheses of Proposition 17.29 hold. For every $\eta_{2}>0$ there are $\bar{s}, \bar{\ell}>0$, depending upon $\eta_{2}, \beta_{2}, \delta_{2}, M_{0}, N_{0}, C_{e}$ and $C_{h}$, such that, if $\varepsilon_{2}$ is sufficiently small, then the following property holds. If $L \in \mathscr{W}_{e}, \ell(L) \leq \bar{\ell}, \Theta(T, q)=Q$ and $\operatorname{dist}\left(\mathbf{p}_{\pi_{0}}(\mathbf{p}(q)), L\right) \leq 4 \sqrt{m} \ell(L)$, then

$$
f_{\mathcal{B}_{\bar{s} \ell(L)}(\mathbf{p}(q))} \mathcal{G}_{\square}(N, Q \llbracket \boldsymbol{\eta} \circ N \rrbracket)^{2} \leq \frac{\eta_{2}}{\ell(L)^{m-2}} \int_{\mathcal{B}_{\ell(L)}(\mathbf{p}(q))}|D N|^{2},
$$

where $\square=s$ if $p=2 Q$ or $\square=$ otherwise.

17.7 Comparison between center manifolds. We list here a final key consequence of the splitting before tilting phenomenon. $\iota_{0, r}$ denotes the map $z \mapsto \frac{z}{r}$.

Proposition 17.32 (Comparing center manifolds). There is a geometric constant $C_{0}$ and a function $\bar{c}_{s}\left(\beta_{2}, \delta_{2}, M_{0}, N_{0}, C_{e}, C_{h}\right)>0$ with the following property. Assume the hypotheses of Proposition $17.29, N_{0} \geq C_{0}, c_{s}:=\frac{1}{64 \sqrt{m}}$ and $\varepsilon_{2}$ is sufficiently small. If for some $r \in] 0,1[$ : 
(a) $\ell(L) \leq c_{s} \rho$ for every $\rho>r$ and every $L \in \mathscr{W}$ with $L \cap B_{\rho}\left(0, \pi_{0}\right) \neq \emptyset$;

(b) $\mathbf{E}^{n o}\left(T, \mathbf{B}_{6 \sqrt{m} \rho}\right)<\varepsilon_{2}$ for every $\rho>r$;

(c) there is $L \in \mathscr{W}$ such that $\ell(L) \geq c_{s} r$ and $L \cap \bar{B}_{r}\left(0, \pi_{0}\right) \neq \emptyset$;

then

(i) the current $T^{\prime}:=\left(\iota_{0, r}\right)_{\sharp} T\left\llcorner\mathbf{B}_{6 \sqrt{m}}\right.$ and the submanifold $\Sigma^{\prime}:=\iota_{0, r}(\Sigma) \cap \mathbf{B}_{7 \sqrt{m}}$ satisfy the assumptions of Theorem 17.24 for some plane $\pi$ in place of $\pi_{0}$;

(ii) for the center manifold $\mathcal{M}^{\prime}$ of $T^{\prime}$ relative to $\pi$ and the $\mathcal{M}^{\prime}$-normal approximation $N^{\prime}$ as in Theorem 17.24, we have

$$
\int_{\mathcal{M}^{\prime} \cap \mathbf{B}_{2}}\left|N^{\prime}\right|^{2} \geq \bar{c}_{s} \max \left\{\mathbf{E}^{n o}\left(T^{\prime}, \mathbf{B}_{6 \sqrt{m}}\right), \mathbf{c}\left(\Sigma^{\prime}\right)^{2}\right\} .
$$

\section{Height bound and first technical lemmas}

We can now discuss the proofs of the main results outlined in the previous section. We begin with $\operatorname{arod}(p)$ version of the sheeting lemma appearing in [DLS16a, Theorem A.1].

Theorem 18.1. Let $p, Q, m, \bar{n}$ and $n$ be positive integers, with $Q \leq \frac{p}{2}$. Then there are $\varepsilon(Q, m, p, \bar{n}, n)>0, \omega(Q, m, p, \bar{n}, n)>0$, and $C_{0}(Q, m, \bar{n}, n)$ with the following property. For $r>0$ and $\mathbf{C}=\mathbf{C}_{r}\left(x_{0}\right)=\mathbf{C}_{r}\left(x_{0}, \pi_{0}\right)$ assume:

(h1) $\Sigma$ and $T$ are as in Assumption 4.1;

(h2) $\partial T\left\llcorner\mathbf{C}=0 \bmod (p),\left(\mathbf{p}_{\pi_{0}}\right) \sharp T\left\llcorner\mathbf{C}=Q \llbracket B_{r}\left(\mathbf{p}_{\pi_{0}}\left(x_{0}\right), \pi_{0}\right) \rrbracket \bmod (p)\right.\right.$, and $E:=\mathbf{E}$ $(T, \mathbf{C})<\varepsilon$.

Then there are $k \in \mathbb{N} \backslash\{0\}$, points $\left\{y_{1}, \ldots, y_{k}\right\} \subset \mathbb{R}^{n}$ and integers $Q_{1}, \ldots, Q_{k}$ such that:

(i) having set $\sigma:=C_{0}\left(E+\mathbf{A}^{2}\right)^{\frac{1}{2 m}}$ and $\rho:=r\left(1-2\left(E+\mathbf{A}^{2}\right)^{\omega}\right)$, the open sets

$$
\mathbf{S}_{i}:=\mathbb{R}^{m} \times\left(y_{i}+\right]-r \sigma, r \sigma\left[^{n}\right)
$$

are pairwise disjoint and

$$
\operatorname{spt}(T) \cap \mathbf{C}_{\rho}\left(x_{0}\right) \subset \bigcup_{i} \mathbf{S}_{i}
$$

(ii) $\left(\mathbf{p}_{\pi_{0}}\right)_{\sharp}\left[T\left\llcorner\left(\mathbf{C}_{\rho}\left(x_{0}\right) \cap \mathbf{S}_{i}\right)\right]=Q_{i} \llbracket B_{\rho}\left(\mathbf{p}_{\pi_{0}}\left(x_{0}\right), \pi_{0}\right) \rrbracket \bmod (p) \forall i \in\{1, \ldots, k\}\right.$, with $Q_{i} \in \mathbb{Z}$. When $Q<\frac{p}{2}$ all $Q_{i}$ must be positive, whereas for $Q=\frac{p}{2}$ either they are all positive or they are all negative; in any case, $\sum_{i}\left|Q_{i}\right|=Q$;

(iii) for every $q \in \operatorname{spt}(T) \cap \mathbf{C}_{\rho}\left(x_{0}\right)$ we have $\Theta(T, q)<\max _{i}\left|Q_{i}\right|+\frac{1}{2}$.

If we keep the same assumptions with $E$ replaced by $E^{n o}:=\mathbf{E}^{n o}(T, \mathbf{C})$, the conclusions hold if we set $\rho:=r\left(1-\eta-2\left(E+\mathbf{A}^{2}\right)^{\omega}\right)$, where $\eta>0$ is any fixed constant (in turn $\varepsilon$ will depend also on $\eta$ ). 
REMARK 18.2. The proof that we are going to present is substantially different from the one in [DLS16a, Theorem A.1], and it could be easily adapted to the case of area minimizing integral currents as well. The statement above is sufficient for our purposes; nonetheless, the proof is actually going to give us more. In particular, in dimension $m \geq 3$ the result holds with a better estimate on the bandwidth of the various $\mathbf{S}_{i}$, namely with $\sigma=C_{0}\left(E+\mathbf{A}^{2}\right)^{\frac{1}{m}}$ in place of $\sigma=C_{0}\left(E+\mathbf{A}^{2}\right)^{\frac{1}{2 m}}$. In dimension $m=2$, the proof below also produces the height bound with the optimal estimate featuring $\sigma=\mathrm{O}\left(E^{1 / 2}\right)$, but only in the cylinder $C_{\frac{r}{2}}\left(x_{0}\right)$.

Proof. In the rest of the proof we denote by $\mathbf{p}$ the orthogonal projection onto $\pi_{0}=$ $\mathbb{R}^{m} \times\{0\}$. The last part of the statement, where $E$ is replaced with $E^{\text {no }}$ follows from Theorem 16.1. Moreover, we assume $x_{0}=0$ and $r=1$ after appropriate translation and rescaling. We also observe, as in the proof of [DLS16a, Theorem A.1] that (iii) is a corollary of the interior monotonicity formula (the only ingredients of the argument in there are the stationarity of the varifold induced by $T_{i}:=T\left\llcorner\left(\mathbf{C}_{\rho} \cap \mathbf{S}_{i}\right)\right.$ and the inequality $\left.\mathbf{M}\left(T_{i}\right) \leq \omega_{m} \rho^{m}\left(\left|Q_{i}\right|+E\right)\right)$.

We therefore focus on (i) and (ii) and since the case $Q<\frac{p}{2}$ is entirely analogous, for the sake of simplicity we assume $Q=\frac{p}{2}$. We first prove (i). We start by considering an approximation as in Proposition 16.2. We thus find an exponent $\omega>0$ (which

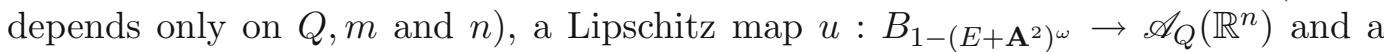
$K \subset B_{1-\left(E+\mathbf{A}^{2}\right)}$ w with the following properties:

(i) $\operatorname{Lip}(u) \leq C\left(E+\mathbf{A}^{2}\right)^{\omega}$;

(ii) $\mathbf{G}_{u}\left\llcorner K \times \mathbb{R}^{n}=T\left\llcorner K \times \mathbb{R}^{n} \bmod (p)\right.\right.$;

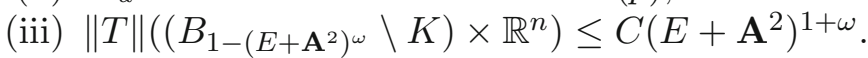

We consider first the case $m>2$. Recall the Poincaré inequality and find a point $T_{0} \in \mathscr{A}_{Q}\left(\mathbb{R}^{n}\right)$ such that

$$
\left(\int_{B_{1-\left(E+\mathbf{A}^{2}\right)^{\omega}}} \mathcal{G}_{s}\left(T_{0}, u(x)\right)^{2^{*}} d x\right)^{1 / 2^{*}} \leq C\|D u\|_{L^{2}\left(B_{1-\left(E+\mathbf{A}^{2}\right) \omega}\right)} \leq C\left(E+\mathbf{A}^{2}\right)^{\frac{1}{2}},
$$

where $2^{*}=\frac{2 m}{m-2}$. Define next the set $K^{*}:=\left\{x \in B_{1-\left(E+\mathbf{A}^{2}\right)^{\omega}}: \mathcal{G}_{s}\left(u(x), T_{0}\right) \leq\right.$ $\left.\bar{C}\left(E+\mathbf{A}^{2}\right)^{\frac{1}{m}}\right\}$, where $\bar{C}$ is a constant which will be later chosen sufficiently large. Using (18.1) and Chebyshev's inequality, we easily conclude

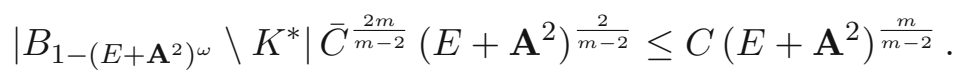

In particular, for any fixed $\bar{\eta}$, if $\bar{C}$ is chosen large enough, we reach the estimate

$$
\left|B_{1-\left(E+\mathbf{A}^{2}\right)^{\omega}} \backslash K^{*}\right| \leq \bar{\eta}\left(E+\mathbf{A}^{2}\right) .
$$

Consider now the set $\bar{K}:=K \cap K^{*}$ and observe that, by choosing $\varepsilon$ sufficiently small, we reach

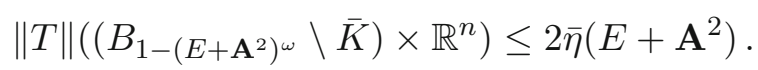


To fix ideas assume now that $T_{0}=\left(\sum_{j=1}^{J} k_{j} \llbracket p_{j} \rrbracket, 1\right)$, where the $p_{j}$ 's are pairwise distinct and all $k_{j}$ are positive. Let $\operatorname{spt}\left(T_{0}\right)=\left\{p_{1}, \ldots p_{J}\right\}$. From (ii) and the definition of $\bar{K}$, it follows easily that $\operatorname{dist}\left(\operatorname{spt}\left(T_{0}\right), \mathbf{p}^{\perp}\left(\operatorname{spt}(\langle T, \mathbf{p}, x)) \leq \bar{C}\left(E+\mathbf{A}^{2}\right)^{\frac{1}{m}}\right.\right.$ for $x \in \bar{K}$. Define thus the sets $\mathbf{U}:=\bigcup_{j}\left\{(x, y):\left|y-p_{j}\right| \leq \bar{C}\left(E+\mathbf{A}^{2}\right)^{\frac{1}{m}}\right\}$ and $\mathbf{U}^{\prime}:=\bigcup_{j}\{(x, y)$ : $\left.\left|y-p_{j}\right| \leq(\bar{C}+1)\left(E+\mathbf{A}^{2}\right)^{\frac{1}{m}}\right\}$, then

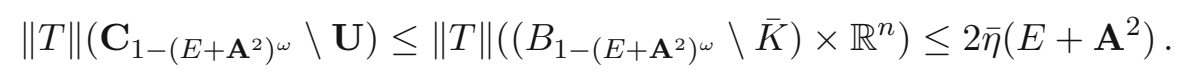

If $q \in \mathbf{C}_{1-2\left(E+\mathbf{A}^{2}\right)^{\omega}} \backslash \mathbf{U}^{\prime}$, then $\mathbf{B}_{\left(E+\mathbf{A}^{2}\right)^{\frac{1}{m}}}(q) \subset \mathbf{C}_{1-\left(E+\mathbf{A}^{2}\right)^{\omega}} \backslash \mathbf{U}$ (we are imposing here $\left.\omega \leq \frac{1}{m}\right)$, and by the monotonicity formula $\|T\|\left(\mathbf{B}_{\left(E+\mathbf{A}^{2}\right)^{\frac{1}{m}}}(q)\right) \geq c_{0}\left(E+\mathbf{A}^{2}\right)$, where $c_{0}$ is a geometric constant. This is however incompatible with (18.5) as soon as $2 \bar{\eta}$ is chosen smaller than $c_{0}$, thus showing that $\operatorname{spt}(T) \cap \mathbf{C}_{1-2\left(E+\mathbf{A}^{2}\right)^{\omega}} \subset \mathbf{U}^{\prime}$. We can now subdivide $\mathbf{U}^{\prime}$ in a finite number of disjoint stripes $\mathbf{S}_{i}$ of width $\tilde{C}\left(E+\mathbf{A}^{2}\right)^{\frac{1}{m}}$, where $\tilde{C}$ is larger than $\bar{C}$ by a factor which depends only on $Q$. This shows therefore the claim (i) of the theorem when $m>2$.

The case $m=2$ is slightly more subtle. Observe first that $|D u|^{2} \leq \min \left\{\mathbf{m}_{c} \mathbf{e}, 1\right\}$ and hence we can use the same argument as in the proof of Theorem 14.3 to achieve

$$
\int_{K}|D u|^{2(1+q)} \leq C E^{1+q-\omega} .
$$

The subtlety is in losing at most $\left(E+\mathbf{A}^{2}\right)^{\omega}$ in the radius of the ball; as usual, the price to pay is a slightly worse estimate, cf. (18.6) with (14.3). Since $\left|B_{1-\left(E+\mathbf{A}^{2}\right)^{\omega}} \backslash K\right| \leq$ $E^{1+\omega}$, if we choose $q$ small enough we easily reach the estimate

$$
\|D u\|_{L^{2+2 q}\left(B_{1-\left(E+\mathbf{A}^{2}\right) \omega}\right)} \leq C E^{\frac{1}{4}} .
$$

In particular, if we set in this case $K^{*}:=\left\{x \in B_{1-\left(E+\mathbf{A}^{2}\right)^{\omega}}: \mathcal{G}_{s}\left(u(x), T_{0}\right) \leq \bar{C}(E+\right.$ $\left.\left.\mathbf{A}^{2}\right)^{\frac{1}{4}}\right\}$ then from Morrey's embedding follows that $K^{*}=B_{1-\left(E+\mathbf{A}^{2}\right)^{\omega}}$, provided $\bar{C}$ is chosen large enough. (18.3) is thus trivially true and the rest of the argument remains unchanged.

We now come to claim (ii). By the constancy theorem, it is easy to see that

$$
\mathbf{p}_{\sharp}\left(T\left\llcorner\mathbf{C}_{1-2\left(E+\mathbf{A}^{2}\right)^{\omega}} \cap \mathbf{S}_{i}\right)=Q_{i} \llbracket B_{1-2\left(E+\mathbf{A}^{2}\right)^{\omega} \rrbracket} \rrbracket \quad \bmod (p),\right.
$$

for some integer $Q_{i} \in\{-(Q-1), \ldots,-1,0,1, \ldots, Q\}$. However, recall that for $x \in \bar{K}$ :

- the support $S$ of the current $Z_{i}(x):=\langle T, \mathbf{p}, x\rangle\left\llcorner\mathbf{C}_{1-2\left(E+\mathbf{A}^{2}\right)^{\omega}} \cap \mathbf{S}_{i}\right.$ consists of at most $Q$ points;

- either all points in $S$ have positive integer multiplicity, or they all have negative integer multiplicity;

- $\mathbf{M}\left(Z_{i}(x)\right) \leq Q$. 
We thus conclude that each $Q_{i}$ is nonzero and that $\left|Q_{i}\right|=\mathbf{M}\left(Z_{i}(x)\right)$. Now, since $\mathbf{M}(\langle T, \mathbf{p}, x\rangle)=Q$, we must have $\sum\left|Q_{i}\right|=Q$. On the other hand

$\sum_{i} \mathbf{p}_{\sharp}\left(T\left\llcorner\mathbf{C}_{1-2\left(E+\mathbf{A}^{2}\right)^{\omega}} \cap \mathbf{S}_{i}\right)=p_{\sharp}\left(T\left\llcorner\mathbf{C}_{1-2\left(E+\mathbf{A}^{2}\right)}\right)=Q \llbracket B_{1-2\left(E+\mathbf{A}^{2}\right)^{\omega}} \rrbracket \quad \bmod (p)\right.\right.$.

Hence $\sum_{i} Q_{i}=Q \bmod (p)$. Hence we conclude that either all $Q_{i}$ 's are positive or they are all negative.

Before coming to the proofs of the Lemmas 17.7 and (17.17), we wish to make the following elementary remark, which will be used throughout the rest of the paper:

Proposition 18.3. There are dimensional constants $\varepsilon(m, n)>0$ and $C(m, n)>0$ with the following property. Consider an oriented $m$-dimensional plane $\pi \subset \mathbb{R}^{m+n}$ and an oriented $(m+d)$-dimensional plane $\Pi \subset \mathbb{R}^{m+n}$, where $d \in\{0, \ldots, n\}$. Let $\pi^{\prime} \subset \Pi$ be an oriented $m$-dimensional plane for which $\left|\pi-\pi^{\prime}\right|=\min _{\tau \subset \Pi}|\pi-\tau|$, and assume $\left|\pi-\pi^{\prime}\right|<\varepsilon$. Then

$$
\left|\pi-\mathbf{p}_{\Pi}(\pi)\right|_{n o}=\left|\pi-\mathbf{p}_{\Pi}(\pi)\right| \leq C\left|\pi-\pi^{\prime}\right| .
$$

In particular:

(Eq) if $\alpha$ and $\beta$ are $m$-dimensional oriented planes of $\mathbb{R}^{m+n}$ for which $|\alpha-\beta|$ is smaller than a positive geometric constant, then $|\alpha-\beta|_{n o}=|\alpha-\beta|$.

The proposition is a simple geometric observation, and its proof is left to the reader.

Proof of Lemma 17.7. The argument given in [DLS16a, Section 4] of [DLS16a, Lemma 1.5] for the existence of the global extension of $\Sigma$ and the minimality of $T^{0}$ in the extended manifold works in our case as well, with straightforward modifications.

We now come to the proof of (17.9), which again follows that given in [DLS16a, Section 4] of [DLS16a, Lemma 1.5], but needs some extra care. First of all, by Assumption 17.5 and Remark 17.6, $\mathbf{A} \leq C_{0} \boldsymbol{m}_{0}^{1 / 2} \leq C_{0}$. Then, by the monotonicity formula, $\left\|T^{0}\right\|\left(\mathbf{B}_{1}\right) \geq c_{0}>0$ and so there is $q \in \operatorname{spt}\left(T^{0}\right) \cap \mathbf{B}_{1}$ such that

$$
\left|\overrightarrow{T^{0}}(q)-\pi_{0}\right|_{n o}^{2} \leq C_{0} \frac{\mathbf{E}^{n o}\left(T^{0}, \mathbf{B}_{1}, \pi_{0}\right)}{\left\|T^{0}\right\|\left(\mathbf{B}_{1}\right)} \leq C_{0} \boldsymbol{m}_{0} .
$$

Now, both $\vec{T}^{0}(q)$ and $-\vec{T}^{0}(q)$ orient a plane contained in $T_{q} \Sigma$. We can thus apply Proposition 18.3 provided $\boldsymbol{m}_{0}$ is sufficiently small. From it we conclude that $\mathbf{p}_{T_{q} \Sigma}\left(\pi_{0}\right)$ is an $m$-dimensional plane with $\left|\mathbf{p}_{T_{q} \Sigma}\left(\pi_{0}\right)-\pi_{0}\right| \leq C_{0} \boldsymbol{m}_{0}^{1 / 2}$. From this inequality we then conclude following literally the final arguments of [DLS16a, Proof of Lemma $1.5]$. 
Proof of Lemma 17.8. We follow the proof of [DLS16a, Lemma 1.6] given in [DLS16a, Section 4]. First of all we notice that, once (17.10) and (17.11) are established, (17.12) follows from Theorem 18.1, since we clearly have that $\mathbf{E}^{n o}\left(T, \mathbf{C}_{11 \sqrt{m} / 2}, \pi_{0}\right) \leq$ $C \mathbf{E}^{n o}\left(T^{0}, \mathbf{B}_{6 \sqrt{m}}, \pi_{0}\right)$. Moreover, recall that there is a set of full measure $A \subset B_{5 \sqrt{m}}$ such that $\left\langle T, \mathbf{p}_{\pi_{0}}, x\right\rangle$ is an integer rectifiable current for every $x \in A$. For any such $x$ we have thus $\left\langle T, \mathbf{p}_{\pi_{0}}, x\right\rangle=\sum_{i}^{J(x)} k_{i}(x) \llbracket p_{i} \rrbracket$ where $p_{1}, \ldots, p_{J(x)}$ is a finite collection of points and each $k_{i}(x)$ is an integer. In particular we must have $\sum_{i} k_{i}(x)=Q \bmod (p)$ and since $1 \leq Q \leq \frac{p}{2}$, at least one $k_{i}(x)$ must be nonzero, which means in turn that $\operatorname{spt}\left(\left\langle T, \mathbf{p}_{\pi_{0}}, x\right\rangle\right) \neq \emptyset$. Hence we conclude that $\operatorname{spt}(T) \cap \mathbf{p}_{\pi_{0}}^{-1}(x) \neq \emptyset$ for every $x \in A$, and by the density of $A$ we conclude that $\operatorname{spt}(T) \cap \mathbf{p}_{\pi_{0}}^{-1}(x) \neq \emptyset$ for every $x \in B_{5 \sqrt{m}}$.

We next come to (17.10) and (17.11). As in the proof of [DLS16a, Lemma 1.6], we argue by contradiction and assume that one among (17.10) and (17.11) fails for a sequence $T_{k}^{0}$ of currents which satisfy Assumption 17.5 with $\varepsilon_{2}=\varepsilon_{2}(k) \downarrow 0$. The compactness property given by Proposition 5.2 ensures the existence of a subsequence, not relabeled, converging to a current $T_{\infty}^{0}$ in the $\mathscr{F}_{K}^{p}$ norm for every compact $K \subset \mathbf{B}_{6 \sqrt{m}}$ : in fact Proposition 5.2 ensures also that $T_{\infty}^{0}$ is area minimizing $\bmod (p)$ in a suitable $(m+\bar{n})$-dimensional plane (the limit of the Riemannian manifolds $\Sigma_{k}$ ) and that the varifolds induced by $T_{k}^{0}$ converge to the varifold induced by $T_{\infty}^{0}$. In particular, $\partial T_{\infty}^{0}=0 \bmod (p)$ in $\mathbf{B}_{6 \sqrt{m}}$ and the tangent plane to $T_{\infty}^{0}$ is parallel to $\pi_{0}$ $\left\|T_{\infty}^{0}\right\|$-almost everywhere.

Observe that by upper semicontinuity of the density, (17.5) implies that 0 is a point of density $Q$ for $T_{\infty}^{0}$. On the other hand (17.6) implies that $\left\|T_{\infty}^{0}\right\|\left(B_{6 \sqrt{m}}\right) \leq$ $Q \omega_{m}(6 \sqrt{m})^{m}$. Hence, by the monotonicity formula, $T_{\infty}^{0}$ must be a cone. Observe that if $q \in \operatorname{spt}\left(T_{\infty}^{0}\right)$ is a point where the approximate tangent space $\pi_{q}$ exists, since $T_{\infty}^{0}$ is a cone, we must have that $q \in \pi_{q}$. Thus $q \in \pi_{0}$ for $\left\|T_{\infty}^{0}\right\|$-a.e. $q$, which in turn implies that $\operatorname{spt}\left(T_{\infty}^{0}\right) \subset \pi_{0}$. In conclusion $T_{\infty}^{0}=Q \llbracket B_{6 \sqrt{m}} \rrbracket \bmod (p)$, and moreover the varifold convergence holds in the whole $\mathbb{R}^{m+n}$.

Again by the monotonicity formula, $\operatorname{spt}\left(T_{k}^{0}\right)$ is converging locally in the sense of Hausdorff to $\operatorname{spt}\left(T_{\infty}^{0}\right)$. In particular if we set $T_{k}:=T_{k}^{0}\left\llcorner\mathbf{B}_{23 \sqrt{m} / 4}\right.$, for $k$ large $T_{k}$ will have no boundary $\bmod (p)$ in $\mathbf{C}_{11 \sqrt{m} / 2}$. Hence it must be (17.11) which fails for an infinite number of $k$ 's. On the other hand we certainly have $\left(\mathbf{p}_{\pi_{0}}\right)_{\sharp} T_{k}\left\llcorner\mathbf{C}_{11 \sqrt{m} / 2}=\right.$ $Q_{k} \llbracket B_{11 \sqrt{m} / 2} \rrbracket \bmod (p)$. Notice that by the varifold convergence we have $\left\|T_{k}^{0}\right\|\left(\mathbf{C}_{11 \sqrt{m} / 2} \backslash\right.$ $\left.\mathbf{B}_{23 \sqrt{m} / 4}\right) \rightarrow 0$ as $k \rightarrow \infty$. In particular the limit of the currents $\left(\mathbf{p}_{\pi_{0}}\right)_{\sharp} T_{k}\left\llcorner\mathbf{C}_{11 \sqrt{m} / 2}\right.$ is the same as the limit of the currents $\left(\mathbf{p}_{\pi_{0}}\right)_{\sharp} T_{k}^{0}\left\llcorner\mathbf{C}_{11 \sqrt{m} / 2}\right.$ and thus it must be $Q_{k}=Q \bmod (p)$ for $k$ large enough.

\section{Tilting of planes and proof of Proposition $\mathbf{1 7 . 1 3}$}

Following [DLS16a], the first important technical step in the proof of the existence of the center manifold is to gain a control on the tilting of the optimal planes as the cubes get refined. The following proposition corresponds to [DLS16a, Proposition 4.1]. 
Proposition 19.1 (Tilting of optimal planes). Assume that the hypotheses of Assumptions 17.5 and 17.10 hold, that $C_{e} \geq C^{\star}$ and $C_{h} \geq C^{\star} C_{e}$, where $C^{\star}\left(M_{0}, N_{0}\right)$ is the constant of the previous section. If $\varepsilon_{2}$ is sufficiently small, then

(i) $\mathbf{B}_{H} \subset \mathbf{B}_{L} \subset \mathbf{B}_{5 \sqrt{m}}$ for all $H, L \in \mathscr{W} \cup \mathscr{S}$ with $H \subset L$.

Moreover, if $H, L \in \mathscr{W} \cup \mathscr{S}$ and either $H \subset L$ or $H \cap L \neq \emptyset$ and $\frac{\ell(L)}{2} \leq \ell(H) \leq \ell(L)$, then the following holds, for $\bar{C}=\bar{C}\left(\beta_{2}, \delta_{2}, M_{0}, N_{0}, C_{e}\right)$ and $C=C\left(\beta_{2}, \delta_{2}, M_{0}, N_{0}\right.$, $\left.C_{e}, C_{h}\right)$ :

(ii) $\left|\hat{\pi}_{H}-\pi_{H}\right| \leq \bar{C} \boldsymbol{m}_{0}^{1 / 2} \ell(H)^{1-\delta_{2}}$;

(iii) $\left|\pi_{H}-\pi_{L}\right| \leq \bar{C} \boldsymbol{m}_{0}^{1 / 2} \ell(L)^{1-\delta_{2}}$;

(iv) $\left|\pi_{H}-\pi_{0}\right| \leq \bar{C} \boldsymbol{m}_{0}^{1 / 2}$;

(v) $\mathbf{h}\left(T, \mathbf{C}_{36 r_{H}}\left(p_{H}, \pi_{0}\right)\right) \leq C \boldsymbol{m}_{0}^{1 / 2 m} \ell(H)$ and $\operatorname{spt}(T) \cap \mathbf{C}_{36 r_{H}}\left(p_{H}, \pi_{0}\right) \subset \mathbf{B}_{H}$;

(vi) For $\pi=\pi_{H}, \hat{\pi}_{H}, \mathbf{h}\left(T, \mathbf{C}_{36 r_{L}}\left(p_{L}, \pi\right)\right) \leq C \boldsymbol{m}_{0}^{1 / 2 m} \ell(L)^{1+\beta_{2}}$ and $\operatorname{spt}(T) \cap \mathbf{C}_{36 r_{L}}\left(p_{L}, \pi\right)$ $\subset \mathbf{B}_{L}$.

In particular, the conclusions of Proposition 17.13 hold.

Proof. First of all we observe that, if we replace (ii), (iii) and (iv) with

(ii)no $\left|\hat{\pi}_{H}-\pi_{H}\right|_{n o} \leq \bar{C} \boldsymbol{m}_{0}^{1 / 2} \ell(H)^{1-\delta_{2}}$,

(iii)no $\left|\pi_{H}-\pi_{L}\right|_{n o} \leq \bar{C} \boldsymbol{m}_{0}^{1 / 2} \ell(L)^{1-\delta_{2}}$, and

(iv)no $\left|\pi_{H}-\pi_{0}\right|_{n o} \leq \bar{C} \boldsymbol{m}_{0}^{1 / 2}$,

then the arguments given in the [DLS16a, Proof of Proposition 4.1] can be followed literally with minor adjustments. Indeed those arguments depend only on:

- the monotonicity formula;

- the triangle inequality $|\alpha-\gamma| \leq|\alpha-\beta|+|\beta-\gamma|$;

- the elementary geometric observation that, for every subset $E$ and every pair of $m$-planes $\alpha$ and $\beta$, we have the inequality

$$
\mathbf{h}(T, E, \alpha) \leq \mathbf{h}(T, E, \beta)+C \operatorname{diam}(E)|\alpha-\beta|,
$$

where $C$ is a geometric constant.

However, it can be easily verified that all such properties remain true if we replace $|\cdot|$ with $|\cdot|_{n o}$.

We next come to (ii), (iii) and (iv). First observe that both $\pi_{H}$ and the (oriented) $m$-plane with the same support and opposite orientation belong to $T_{p_{H}} \Sigma$. For this reason, the definition of $\pi_{H}$ implies that $\left|\pi_{H}-\hat{\pi}_{H}\right|_{n o}=\left|\pi_{H}-\hat{\pi}_{H}\right|$, thus allowing us to infer (ii) from (ii)no.

Next, recall that we have $\left|\hat{\pi}_{H}-\pi_{0}\right|=\left|\hat{\pi}_{H}-\pi_{0}\right|_{n o}$, cf. Remark 17.4. Hence (iv)no implies (iv). Now, combining (iv) for two planes $H$ and $L$ as in statement (iii) of the proposition, we conclude that $\left|\pi_{H}-\pi_{L}\right| \leq\left|\pi_{H}-\pi_{0}\right|+\left|\pi_{L}-\pi_{0}\right| \leq C \boldsymbol{m}_{0}^{1 / 2}$. Hence, again assuming that $\varepsilon_{2}$ is sufficiently small, we can apply Proposition 18.3, in particular conclusion (Eq): $\left|\pi_{H}-\pi_{L}\right|=\left|\pi_{H}-\pi_{L}\right|_{n o}$. Thus (iii) is a consequence of (iii)no. 
REMARK 19.2. Notice that, even though our arguments use the nonoriented excess as control parameter, the estimates of Proposition 19.1 on the tilt of optimal planes which are needed for the construction of the center manifold involve a measure of the classical distance between oriented planes. As seen in the proof, such estimates continue to be valid in our setting thanks to our choice of optimal planes made in (17.3) and to the observation made in Proposition 18.3.

Arguing as in [DLS16a, Section 4.3] we get the following existence theorem with very minor modifications (the only adjustment that needs to be taken into consideration is that the identities [DLS16a, (4.9)], [DLS16a, (4.10)] and the subsequent analogous ones must be replaced with the same equalities $\bmod (p))$ :

Proposition 19.3 (Existence of interpolating functions). Assume the conclusions of the Proposition 19.1 apply. The following facts are true provided $\varepsilon_{2}$ is sufficiently small. Let $H, L \in \mathscr{W} \cup \mathscr{S}$ be such that either $H \subset L$ or $H \cap L \neq \emptyset$ and $\frac{\ell(L)}{2} \leq$ $\ell(H) \leq \ell(L)$. Then,

(i) For $\pi=\pi_{H}, \hat{\pi}_{H},\left(\mathbf{p}_{\pi}\right)_{\sharp} T\left\llcorner\mathbf{C}_{32 r_{L}}\left(p_{L}, \pi\right)=Q \llbracket B_{32 r_{L}}\left(p_{L}, \pi\right)\right) \rrbracket \bmod (p)$ and $T$ satisfies the assumptions of 16.1 in the cylinder $\mathbf{C}_{32 r_{L}}\left(p_{L}, \pi\right)$.

(ii) Let $f_{H L}$ be the $\pi_{H}$-approximation of $T$ in $\mathbf{C}_{8 r_{L}}\left(p_{L}, \pi_{H}\right)$ and $h_{H L}:=\left(\boldsymbol{\eta} \circ f_{H L}\right) *$ $\varrho_{\ell(L)}$ be its smoothed average. Set $\varkappa_{H}:=\pi_{H}^{\perp} \cap T_{p_{H}} \Sigma$ and consider the maps

$$
\begin{aligned}
& x \mapsto \bar{h}(x) \quad:=\mathbf{p}_{T_{p_{H}} \Sigma}(h) \quad \in \varkappa_{H} \\
& x \mapsto h_{H L}(x):=\left(\bar{h}(x), \Psi_{p_{H}}(x, \bar{h}(x))\right) \in \varkappa_{H} \times\left(T_{p_{H}}(\Sigma)\right)^{\perp} .
\end{aligned}
$$

Then there is a smooth $g_{H L}: B_{4 r_{L}}\left(p_{L}, \pi_{0}\right) \rightarrow \pi_{0}^{\perp}$ s.t. $\mathbf{G}_{g_{H L}}=\mathbf{G}_{h_{H L}}\left\llcorner\mathbf{C}_{4 r_{L}}\left(p_{L}, \pi_{0}\right)\right.$.

Definition 19.4. $h_{H L}$ and $g_{H L}$ will be called, respectively, tilted $(H, L)$-interpolating function and $(H, L)$-interpolating function.

Observe that the tilted $(L, L)$-interpolating function and the $(L, L)$-interpolating function correspond to the tilted $L$-interpolating function and to the $L$-interpolating function of Definition 17.18. Obviously, Lemma 17.17 is just a particular case of Proposition 19.3. As in Definition 17.18, we will set $h_{L}:=h_{L L}$ and $g_{L}:=g_{L L}$.

\section{The key construction estimates}

Having at disposal the Existence Proposition 19.3 we can now come to the main estimates on the building blocks of the center manifold, which in fact correspond precisely to [DLS16a, Proposition 4.4] and are thus restated here only for the reader's convenience.

Proposition 20.1. (Construction estimates) Assume the conclusions of Propositions 19.1 and 19.3 apply and set $\kappa=\min \left\{\beta_{2} / 4, \varepsilon_{0} / 2\right\}$. Then, the following holds for any pair of cubes $H, L \in \mathscr{P}^{j}$ (cf. Definition 17.18), where $C=C\left(\beta_{2}, \delta_{2}, M_{0}, N_{0}, C_{e}, C_{h}\right)$ : 
(i) $\left\|g_{H}\right\|_{C^{0}(B)} \leq C \boldsymbol{m}_{0}^{1 / 2 m}$ and $\left\|D g_{H}\right\|_{C^{2, \kappa}(B)} \leq C \boldsymbol{m}_{0}^{1 / 2}$, for $B=B_{4 r_{H}}\left(x_{H}, \pi_{0}\right)$;

(ii) if $H \cap L \neq \emptyset$, then $\left\|g_{H}-g_{L}\right\|_{C^{i}\left(B_{r_{L}}\left(x_{L}, \pi_{0}\right)\right)} \leq C \boldsymbol{m}_{0}^{1 / 2} \ell(H)^{3+\kappa-i}$ for every $i \in$ $\{0, \ldots, 3\}$;

(iii) $\left|D^{3} g_{H}\left(x_{H}\right)-D^{3} g_{L}\left(x_{L}\right)\right| \leq C \boldsymbol{m}_{0}^{1 / 2}\left|x_{H}-x_{L}\right|^{\kappa}$;

(iv) $\left\|g_{H}-y_{H}\right\|_{C^{0}} \leq C \boldsymbol{m}_{0}^{1 / 2 m} \ell(H)$ and $\left|\pi_{H}-T_{\left(x, g_{H}(x)\right)} \mathbf{G}_{g_{H}}\right| \leq C \boldsymbol{m}_{0}^{1 / 2} \ell(H)^{1-\delta_{2}} \forall x \in$ $H$;

(v) if $L^{\prime}$ is the cube concentric to $L \in \mathscr{W}^{j}$ with $\ell\left(L^{\prime}\right)=\frac{9}{8} \ell(L)$, then

$$
\left\|\varphi_{i}-g_{L}\right\|_{L^{1}\left(L^{\prime}\right)} \leq C \boldsymbol{m}_{0} \ell(L)^{m+3+\beta_{2} / 3} \text { for all } i \geq j .
$$

The proof of Theorem 17.19 assuming the validity of Proposition 20.1 is given in [DLS16a, Section 4.4, Proof of Theorem 1.17]. As for the proof of Proposition 20.1, we discuss briefly why the arguments given in [DLS16a, Section 5] apply in our case as well. First of all, the key tool in the proof, namely [DLS16a, Proposition 5.2], is valid under our assumptions for the following reason. The proof given in [DLS16a, Section 5.1] is based on the following facts:

- The first variation of $T$ vanishes, and this allows to estimate the first variation of $\mathbf{G}_{f}=\mathbf{G}_{f_{H L}}$ as in [DLS16a, Eq. (5.4)].

- The estimates claimed in [DLS16a, Eqs. (5.5)-(5.9)] are valid because of Theorem 16.1 and the Taylor expansion of [DLHMS, Corollary 13.2].

- Using the decomposition $\delta \mathbf{G}_{f}=\delta\left(\mathbf{G}_{f^{+}}\left\llcorner B_{+}\right)+\delta\left(\mathbf{G}_{f^{-}}\left\llcorner B_{-}\right)+Q \delta\left(\mathbf{G}_{\boldsymbol{\eta} \circ f}\left\llcorner B_{0}\right)\right.\right.\right.$ we can show the validity of [DLS16a, Eq. (5.11)].

The three ingredients above are then used to show the first estimate of [DLS16a, Proposition 5.2], namely [DLS16a, Eq. (5.1)]. The derivation of the remaining part of [DLS16a, Proposition 5.2] is then a pure PDE argument based only on [DLS16a, Eq. (5.1)].

In [DLS16a, Section 5.2] the [DLS16a, Proposition 4.4] is used to derive [DLS16a, Lemma 5.3], which in fact includes the conclusions (i) and (ii) of Proposition 20.1. This derivation does not depend anymore on the underlying current and thus the proof given in [DLS16a, Section 5.2] works literally in our case as well. The remaining part of Proposition 20.1 is derived from [DLS16a, Lemma 5.5]. The latter is based solely on the estimates on the Lipschitz approximation (which are provided by Theorem 16.1) and on [DLS16a, Lemma 5.5], whose role is taken, in our setting, by [DLHMS, Lemma 16.1].

\section{Existence and estimates on the $\mathcal{M}$-normal approximation}

Corollary 17.22 can be proved following the argument of [DLS16a, Section 6.1]. The only adjustement needed is in the argument for claim (iii). Following the one of [DLS16a, Section 6.1] we conclude that at every $q \in \Phi(\Gamma)$, if we denote by $\pi$ the oriented tangent plane to $\mathcal{M}$ at $q$, then the current $Q \llbracket \pi \rrbracket$ is the unique tangent 
$\bmod (p)$ of $T$ at $q$, in the sense of Corollary 7.3. We then can use Proposition 5.2 to conclude that $\Theta(T, q)=Q$.

For Theorem 17.24 we can repeat the arguments of [DLS16a, Section 6.2] in order to prove the existence of the $\mathcal{M}$-normal approximation and the validity of (17.22) and (17.23). As for (17.25) we can repeat the arguments of [DLS16a, Section 6.3], whereas in order to get (17.24) we make the following adjustments to the first part of [DLS16a, Section 6.3]. The paragraphs leading to [DLS16a, Eq. (6.11)] are obviously valid in our setting. However [DLS16a, Eq. (6.11)] must be replaced with the following analogous estimate

$$
\begin{aligned}
\int_{\mathbf{p}^{-1}(\mathcal{L})} \mid \overrightarrow{\mathbf{T}}_{F}(x) & -\left.\overrightarrow{\mathcal{M}}(\mathbf{p}(x))\right|_{n o} ^{2} d\left\|\mathbf{T}_{F}\right\|(x) \\
\leq & \int_{\mathbf{p}^{-1}(\mathcal{L})}|\vec{T}(x)-\overrightarrow{\mathcal{M}}(\mathbf{p}(x))|_{n o}^{2} d\|T\|(x)+C \boldsymbol{m}_{0}^{1+\gamma_{2}} \ell(L)^{m+2+\gamma_{2}} \\
\leq & \int_{\mathbf{p}^{-1}(\mathcal{L})}\left|\vec{T}(x)-\vec{\pi}_{L}\right|_{n o}^{2} d\|T\|(x)+C \boldsymbol{m}_{0} \ell(L)^{m+2-2 \delta_{2}}
\end{aligned}
$$

From this one we proceed as in the rest of [DLS16a, Section 6.3] using the Taylor expansion of [DLHMS, Proposition 13.3] in place of [DLS15, Proposition 3.4].

\section{Separation and splitting before tilting}

The arguments for Proposition 17.26 and Corollary 17.27 can be taken from [DLS16a, Section 7.1], modulo using Theorem 18.1 in place of [DLS16a, Theorem A.1].

We next come to the proof of Proposition 17.29. A first important ingredient is the Unique continuation property of [DLS16a, Lemma 7.1], which we will now prove it is valid for $\mathscr{A}_{Q}\left(\mathbb{R}^{n}\right)$ minimizers as well.

Lemma 22.1 (Unique continuation for Dir-minimizers). For every $\eta \in(0,1)$ and $c>0$, there exists $\gamma>0$ with the following property. If $w: \mathbb{R}^{m} \supset B_{2 r} \rightarrow \mathscr{A}_{Q}\left(\mathbb{R}^{n}\right)$ is Dir-minimizing, $\operatorname{Dir}\left(w, B_{r}\right) \geq c$ and $\operatorname{Dir}\left(w, B_{2 r}\right)=1$, then

$$
\operatorname{Dir}\left(w, B_{s}(q)\right) \geq \gamma \quad \text { for every } B_{s}(q) \subset B_{2 r} \text { with } s \geq \eta r .
$$

Proof. We follow partially the argument of [DLS16a, Section 7.2] for [DLS16a, Lemma 7.1]. In particular, the second part of the argument, which reduces the statement to the following claim, can be applied with no alterations:

(UC) if $\Omega$ is a connected open set and $w \in W^{1,2}\left(\Omega, \mathscr{A}_{Q}\left(\mathbb{R}^{n}\right)\right)$ is Dir-minimizing in any every bounded $\Omega^{\prime} \subset \subset \Omega$, then either $w$ is constant or $\int_{J}|D w|^{2}>0$ for every nontrivial open $J \subset \Omega$.

However, the proof given in [DLS16a, Section 7.1] of (UC) when $w \in W^{1,2}\left(\Omega, \mathcal{A}_{Q}\left(\mathbb{R}^{n}\right)\right)$ cannot be repeated in our case, since it uses heavily the fact that the singular sets of $\mathcal{A}_{Q}\left(\mathbb{R}^{n}\right)$-valued Dir-minimizers cannot disconnect the domain, a property which 
is not enjoyed by $\mathscr{A}_{Q}\left(\mathbb{R}^{n}\right)$-valued Dir-minimizers. We thus have to modify the proof somewhat, although the tools used are essentially the same.

Assume by contradiction that there are a connected open set $\Omega \subset \mathbb{R}^{m}$, a map $w \in W_{l o c}^{1,2}\left(\Omega, \mathscr{A}_{Q}\left(\mathbb{R}^{n}\right)\right)$ and a nontrivial open subset $J \subset \Omega$ such that

(a) $w$ is Dir-minimizing on every open $\Omega^{\prime} \subset \subset \Omega$;

(b) $w$ is not constant, and thus $\int_{\Omega^{\prime}}|D w|^{2}>0$ for some $\Omega^{\prime} \subset \subset \Omega$;

(c) $\int_{J}|D w|^{2}=0$.

Observe first that, from the classical unique continuation of harmonic functions, either $\boldsymbol{\eta} \circ \boldsymbol{w}$ is constant, or it has positive Dirichlet energy on any nontrivial open subset of $\Omega$. Since however the Dirichlet energy of $\boldsymbol{\eta} \circ w$ is controlled from above by that of $w$, (c) excludes the second posssibility. Thus $\eta \circ w$ is constant and hence, without loss of generality, we can assume $\boldsymbol{\eta} \circ \boldsymbol{w} \equiv 0$.

Next assume, without loss of generality, that $J$ is connected. Clearly, $w$ is constantly equal to some $P \in \mathscr{A}_{Q}\left(\mathbb{R}^{n}\right)$ on $J$. Since, without loss of generality, we could "flip the signs of the Dirac masses" which constitute the values of $u$, we can always assume that $P=\left(\sum_{i} \llbracket P_{i} \rrbracket, 1\right)$. We then distinguish two cases.

First Case. The diameter of $\operatorname{spt}(P)$ is positive, namely $\left|P_{i}-P_{j}\right|>0$ for some $i \neq j$. In this case consider the interior $U$ of the set $\{w=P\}$. We want to argue that $U=\Omega$, which contradicts (b). Since $\Omega$ is open and connected, it suffices to show that $\partial U \cap \Omega=\emptyset$. In order to show this, consider a point $x \in \partial U$. If $x \in \Omega$, using the continuity of the map $w$, we know that in a sufficiently small ball $B_{\rho}(x)$ there is an $\mathcal{A}_{Q}\left(\mathbb{R}^{n}\right)$-valued map $z$ such that $w(y)=(z(y), 1)$ for all $y \in B_{\rho}(x)$. As such, $z$ must be a Dir-minimizer to which we can apply [DLS16a, Section 7.2]: since $\int_{J^{\prime}}|D z|^{2}=0$ for some nontrivial open $J^{\prime} \subset B_{\rho}(x)$, we must have that $z$ is constant on $B_{\rho}(x)$. But then we would have $B_{\rho}(x) \subset U$, thus contradicting the assumption that $x \in \partial U$.

Second Case. The remaining possibility is that $P=Q \llbracket \boldsymbol{\eta} \circ w(x) \rrbracket=Q \llbracket 0 \rrbracket($ which equals both $(Q \llbracket 0 \rrbracket, 1)$ and $(Q \llbracket 0 \rrbracket,-1)$, since the latter points are identified in $\left.\mathscr{A}_{Q}\left(\mathbb{R}^{n}\right)\right)$. Define therefore

$$
K:=\{w=Q \llbracket 0 \rrbracket\},
$$

and (since $K \supset J$ ) observe that $|K|>0$. Consider now the set $\tilde{K}$ of points $x \in \mathbb{R}^{m}$ such that

$$
0<\lim _{k \rightarrow \infty} \frac{\left|K \cap B_{r_{k}}(x)\right|}{\omega_{m} r_{k}^{m}}<1 \quad \text { for some } r_{k} \downarrow 0^{+},
$$

and notice that $\tilde{K} \subset K$ since $w$ is continuous. The set $\tilde{K}$ is necessarily nonempty. If it were empty, we could first apply the classical characterization of Federer of sets of finite perimeter, cf. [Fed69, Theorem 4.5.11], to infer that $K$ is a set of finite perimeter, and subsequently we could then apply the classical structure theorem of De Giorgi to conclude that, since the reduced boundary of $K$ would be empty, $D \mathbf{1}_{K}=0$. The latter would imply that $\mathbf{1}_{K}$ is constant on the connected set $\Omega$, namely that $\Omega \backslash K$ has zero Lebesgue measure, which in turn would contradict (b). 
Fix a point $x \in \tilde{K}$. Clearly it must be $\int_{B_{\rho}(x)}|D w|^{2}>0$ for every $\rho>0$, otherwise $w$ would be constant in a neighborhood of $x$ and thus $x$ would be an interior point of $K$. Denoting $I_{x, w}(\cdot)$ the frequency function of $w$ at $x$ as in [DLHMS, Definition 9.1], from [DLHMS, Theorem 9.2] we must then have

$$
\infty>I_{0}:=\lim _{r \downarrow 0} I_{x, w}(r)>0 .
$$

Define then the maps $y \mapsto w_{r}(y)$, whose positive and negative parts are given by

$$
w_{r}^{ \pm}(y):=\sum_{i} \llbracket r^{-I_{0}} w_{i}^{ \pm}(r y+x) \rrbracket
$$

and observe that a subsequence of $\left\{w_{r_{k}}\right\}_{k \in \mathbb{N}}$, not relabeled, is converging to a nontrivial $w_{0} \in W_{\text {loc }}^{1,2}\left(\mathbb{R}^{m}, \mathscr{A}_{Q}\left(\mathbb{R}^{n}\right)\right)$ which minimizes the Dirichlet energy on every $\Omega^{\prime} \subset \subset \mathbb{R}^{m}$ and is $I_{0}$-homogeneous.

Next define the sets $K_{r_{k}}:=r_{k}^{-1}(K-x)$, where the maps $w_{r_{k}}$ vanish identically, and observe that, by (22.1), $\liminf _{k}\left|K_{r_{k}} \cap \bar{B}_{1}\right|>0$. Since the sets $K_{r_{k}} \cap \bar{B}_{1}$ are compact we can, without loss of generality, assume that they convergence in the sense of Hausdorff to some set $K_{0}$. The limiting map $w_{0}$ vanishes on such set because the $w_{r_{k}}$ are converging locally uniformly to $w_{0}$. On the other hand it is elementary to see that the Lebesgue measure is upper semicontinuous under Hausdorff convergence and we thus conclude $\left|K_{0}\right|>0$.

We can now repeat the procedure above on some point $y \neq 0$ where the Lebesgue density of $K_{0}$ does not exist or it is neither zero nor one. We find thus a corresponding tangent function $w_{1}$ that has all the properties of $w_{0}$, namely

- it is nontrivial,

- it vanishes identically on a set of positive measure,

- it is $I_{1}$-homogeneous for some positive constant $I_{1}$,

- and it minimizes the Dirichlet energy on any bounded open set.

In addition $w_{1}$ is invariant under translations along the direction $\frac{y}{|y|}$. Assuming, after rotations, that such vector is $e_{m}=(0,0, \ldots, 0,1)$, the function $w_{1}$ depends therefore only on the variables $x_{1}, \ldots, x_{m-1}$ and can thus be treated as a function defined over $\mathbb{R}^{m-1}$. Iterating $m-2$ more times such procedure we achieve finally a function $w_{m-1}: \mathbb{R} \rightarrow \mathscr{A}_{Q}\left(\mathbb{R}^{n}\right)$ with the following properties:

(A) $w_{m-1}$ is identically $Q \llbracket 0 \rrbracket$ on some set of positive measure;

(B) $\int_{1}^{-1}\left|D w_{m-1}\right|^{2}>0$;

(C) $w_{m-1}$ is Dir-minimizing on $] a, b[$ for every $0<a<b<\infty$;

(D) $w_{m-1}$ is $\alpha$-homogeneous for some positive $\alpha>0$;

(E) $\boldsymbol{\eta} \circ w_{m-1} \equiv 0$.

Because of $(\mathrm{A})$ and $(\mathrm{D}), w_{m-1}$ must be identically equal to $Q \llbracket 0 \rrbracket$ on at least one of two half-lines $]-\infty, 0]$ and $[0, \infty[$. Without loss of generality we can assume this 
happens on the $]-\infty, 0\left[\right.$. Let now $w_{m-1}(1)=\left(\sum_{i} \llbracket c_{i} \rrbracket, \varepsilon\right)$, where $\varepsilon \in\{-1,1\}$. By (D) we then have

$$
w_{m-1}(x)=\left(\sum_{i} \llbracket c_{i} x^{\alpha} \rrbracket, \varepsilon\right) \quad \forall x \geq 0
$$

Observe that, because of $(\mathrm{B})$, at least one of the $c_{i}$ 's is nonzero. Therefore $\varepsilon$ cannot be equal to 1 , otherwise $w_{m-1}$ would give an $\mathcal{A}_{Q}\left(\mathbb{R}^{n}\right)$-valued Dir-minimizer on the real line with a singularity, which is not possible. However, since $(Q \llbracket 0 \rrbracket, 1)=$ $(Q \llbracket 0 \rrbracket,-1)$, if $\varepsilon$ equals -1 we reach precisely the same contradiction. This completes the proof.

We keep following the strategy of [DLS16a, Section 7.2] towards a proof of Proposition 17.29. First of all, we introduce some useful notation.

Definition 22.2. Let $w: E \rightarrow \mathscr{A}_{Q}\left(\mathbb{R}^{n}\right)$, let $E_{+}, E_{-}$and $E_{0}$ be the canonical decomposition of $E$ induced by $w$ and let $w^{+}, w^{-}$and $\boldsymbol{\eta} \circ w$ the corresponding maps, as in [DLHMS, Definition 2.7]. For any $f: E \rightarrow \mathbb{R}^{n}$ we denote by $w \oplus f$ (resp. $w \ominus f$ ) the $\mathscr{A}_{Q}\left(\mathbb{R}^{n}\right)$-valued map which

- on $E_{+}$coincides with $\left(w^{+} \oplus f, 1\right)$ (resp. $\left.\left(w^{+} \ominus f, 1\right)\right)$,

- on $E_{-}$coincides with $\left(w^{-} \oplus f,-1\right)$ (resp. $\left.\left(w^{-} \ominus f,-1\right)\right)$,

- and on $E_{0}$ coincides with $Q \llbracket \boldsymbol{\eta} \circ w+f \rrbracket$ (resp. $Q \llbracket \boldsymbol{\eta} \circ w-f \rrbracket$.

Moreover we use the shorthand notation $\bar{w}$ for $w \ominus \boldsymbol{\eta} \circ w$.

We next show that if the energy of an $\mathscr{A}_{Q}\left(\mathbb{R}^{n}\right)$-valued Dir-minimizer $w$ does not decay appropriately, then the map must "split", in other words $\bar{w}$ cannot be too small compared to $\boldsymbol{\eta} \circ w$. As in [DLS16a, Section 7.2], we fix $\lambda>0$ such that

$$
(1+\lambda)^{(m+2)}<2^{\delta_{2}}
$$

and we claim the following analog of [DLS16a, Proposition 7.2].

Proposition 22.3 (Decay estimate for Dir-minimizers). For every $\eta>0$, there is $\gamma>0$ with the following property. Let $w: \mathbb{R}^{m} \supset B_{2 r} \rightarrow \mathscr{A}_{Q}\left(\mathbb{R}^{n}\right)$ be Dir-minimizing in every $\Omega^{\prime} \subset \subset B_{2 r}$ such that

$$
\int_{B_{(1+\lambda) r}} \mathcal{G}_{s}(D w, Q \llbracket D(\boldsymbol{\eta} \circ w)(0) \rrbracket)^{2} \geq 2^{\delta_{2}-m-2} \operatorname{Dir}\left(w, B_{2 r}\right) .
$$

Then, if we let $\bar{w}$ be as in Definition 22.2, the following holds:

$\gamma \operatorname{Dir}\left(w, B_{(1+\lambda) r}\right) \leq \operatorname{Dir}\left(\bar{w}, B_{(1+\lambda) r}\right) \leq \frac{1}{\gamma r^{2}} \int_{B_{s}(q)}|\bar{w}|^{2} \quad \forall B_{s}(q) \subset B_{2 r}$ with $s \geq \eta r$. 
The proof of [DLS16a, Proposition 7.2] can be literally followed for our case using the Unique continuation Lemma 22.1 in combination with the next simple algebraic computation (which is the counterpart of [DLS16a, Lemma 7.3]).

Lemma 22.4. Let $B \subset \mathbb{R}^{m}$ be a ball centered at $0, w \in W^{1,2}\left(B, \mathscr{A}_{Q}\left(\mathbb{R}^{n}\right)\right)$ Dirminimizing and $\bar{w}$ as in Definition 22.2 We then have

$$
Q \int_{B}|D(\boldsymbol{\eta} \circ w)-D(\boldsymbol{\eta} \circ w)(0)|^{2}=\int_{B} \mathcal{G}_{s}(D w, Q \llbracket D(\boldsymbol{\eta} \circ w)(0) \rrbracket)^{2}-\operatorname{Dir}(\bar{w}, B) .
$$

The detail of the necessary modifications to the argument in [DLS16a, Proof of Proposition 7.2] towards proving Proposition 22.3 are left to the reader; we will instead show how to prove the lemma above.

Proof. Let $u:=\boldsymbol{\eta} \circ w$ and observe that it is harmonic. Thus, using the mean value property of harmonic functions and a straightforward computation we get

$$
Q \int_{B}|D u-D u(0)|^{2}=Q \int_{B}|D u|^{2}-Q|B||D u(0)|^{2} .
$$

On the other hand, using again the mean value property of harmonic functions, it is easy to see that

$$
\int_{B} \mathcal{G}_{s}(D w, Q \llbracket D u(0) \rrbracket)^{2}=\sum_{\varepsilon=+,-} \int_{B^{\varepsilon}} \mathcal{G}\left(D w^{\varepsilon}, Q \llbracket D u(0) \rrbracket\right)^{2}+Q \int_{B_{0}}|D u-D u(0)|^{2}
$$

and

$$
\int_{B^{\varepsilon}} \mathcal{G}\left(D w^{\varepsilon}, Q \llbracket D u(0) \rrbracket\right)^{2}=\int_{B^{\varepsilon}}\left(\left|D w^{\varepsilon}\right|^{2}-2 Q D u: D u(0)+Q|D u(0)|^{2}\right) .
$$

In particular, we get

$$
\int_{B} \mathcal{G}_{s}(D w, Q \llbracket D u(0) \rrbracket)^{2}=\int_{B}|D w|^{2}+Q|B||D u(0)|^{2}-2 Q D u(0): \int_{B} D u
$$

and again by the mean value property we conclude

$$
\int_{B} \mathcal{G}_{s}(D w, Q \llbracket D u(0) \rrbracket)^{2}=\int_{B}|D w|^{2}-Q|B||D u(0)|^{2} .
$$

Combining (22.6) and (22.7) we thus get

$$
\begin{aligned}
\int_{B} & \mathcal{G}_{s}(D w, Q \llbracket D(\boldsymbol{\eta} \circ w)(0) \rrbracket)^{2}-Q \int_{B}|D(\boldsymbol{\eta} \circ w)-D(\boldsymbol{\eta} \circ w)(0)|^{2} \\
= & \int_{B} \mathcal{G}_{s}(D w, Q \llbracket D u(0) \rrbracket)^{2}-Q \int_{B}|D u-D u(0)|^{2}=\int_{B}|D w|^{2}-Q \int_{B}|D u|^{2} \\
= & \int_{B}|D w|^{2}-Q \int_{B}|D(\boldsymbol{\eta} \circ w)|^{2} .
\end{aligned}
$$


Next, a simple algebraic computations shows

$$
\begin{aligned}
\int_{B}|D w|^{2} & =\sum_{\varepsilon=+,-} \int_{B^{\varepsilon}}\left|D w^{\varepsilon}\right|^{2}+Q \int_{B_{0}}|D(\boldsymbol{\eta} \circ w)|^{2} \\
& =\sum_{\varepsilon=+,-}\left(\int_{B^{\varepsilon}}\left|D \bar{w}^{\varepsilon}\right|^{2}+Q\left|D\left(\boldsymbol{\eta} \circ w^{\varepsilon}\right)\right|^{2}\right)+Q \int_{B_{0}}|D(\boldsymbol{\eta} \circ w)|^{2} \\
& =\int_{B}|D \bar{w}|^{2}+Q \int_{B}|D(\boldsymbol{\eta} \circ w)|^{2} .
\end{aligned}
$$

Clearly, (22.8) and (22.9) give (22.5) and conclude the proof.

Proof of Proposition 17.29. Having at hand the analogs of the tools used in [DLS16a, Section 7.3], we can following the argument given there for [DLS16a, Proposition 3.4]. In the first step of the proof (namely [DLS16a, Step 1, p. 548]) we use [DLHMS, Corollary 13.2] in place of [DLS15, Corollary 3.3], we use Theorem 16.1 in place of [DLS14, Theorem 2.4] and we replace $\mathbf{E}$ with $\mathbf{E}^{\text {no }}$ in the various formulas. We also replace $\mathcal{G}$ with $\mathcal{G}_{s}$ in case $p=2 Q$. We then follow [DLS16a, Step 2, p. 550], where we use Lemma 22.1 and Proposition 22.3 in place of [DLS16a, Lemma $7.1 \&$ Proposition 7.2] in case $p=2 Q$. In the final [DLS16a, Step 3, p. 551] we use the reparametrization Theorem [DLHMS, Theorem 15.1] in place of the corresponding [DLS15, Theorem 5.1] and measure the distance between $m$-planes using $|\cdot|_{\text {no }}$ in place of $|\cdot|$.

\section{Persistence of multiplicity $Q$ points}

The proofs of Proposition 17.30 and Proposition 17.31 can be easily adapted to our case from [DLS16a, Proofs of Proposition 3.5 \& Proposition 3.6] once we prove the following analog of [DLS14, Theorem 2.7]:

Theorem 23.1 (Persistence of $Q$-points). For every $\hat{\delta}, C^{\star}>0$, there is $\left.\bar{s} \in\right] 0, \frac{1}{2}[$ such that, for every $s<\bar{s}$, there exists $\hat{\varepsilon}\left(s, C^{*}, \hat{\delta}\right)>0$ with the following property. If $T$ is as in Theorem 16.1, $E^{n o}:=\mathbf{E}^{n o}\left(T, \mathbf{C}_{4 r}(x)\right)<\hat{\varepsilon}, r^{2} \mathbf{A}^{2} \leq C^{\star} E^{n o}$ and $\Theta(T,(p, q))=Q$ at some $(p, q) \in \mathbf{C}_{r / 2}(x)$, then the approximation $f$ of Theorem 15.1 satisfies

$$
\int_{B_{s r}(p)} \mathcal{G}_{\square}(f, Q \llbracket \boldsymbol{\eta} \circ f \rrbracket)^{2} \leq \hat{\delta} s^{m} r^{2+m} E^{n o},
$$

where $\square=s$ if $p=2 Q$ or $\square=$ otherwise.

In order to show Theorem 23.1 we can follow literally [DLS14, Section 9]. Indeed the proof in [DLS14, Section 9] relies on the Hölder estimates for Dir minimizers (which are valid in the $\mathscr{A}_{Q}\left(\mathbb{R}^{n}\right)$ case by [DLHMS, Theorem 8.1]), the estimates on the Lipschitz approximation (given by Theorem 16.1 and the classical monotonicity formula in the slightly improved version of [DLS14, Lemma A.1]. Although the latter is stated for stationary integral currents in a Riemannian manifold, it is easy to see 
that the proof is in fact valid for stationary varifolds and as such can be applied to $\bmod (p)$ area-minimizing currents. We formulate the precise theorem here for the reader's convenience.

LEMma 23.2. There is a constant $C$ depending only on $m, n$ and $\bar{n}$ with the following property. If $\Sigma \subset \mathbb{R}^{m+n}$ is a $C^{2}(m+\bar{n})$-dimensional submanifold with $\left\|A_{\Sigma}\right\|_{\infty} \leq \mathbf{A}, U$ is an open set in $\mathbb{R}^{m+n}$ and $V$ an $m$-dimensional integral varifold supported in $\Sigma$ which is stationary in $\Sigma \cap U$, then for every $\xi \in \Sigma \cap U$ the function $\rho \mapsto \exp \left(C \mathbf{A}^{2} \rho^{2}\right) \rho^{-m}\|V\|\left(\mathbf{B}_{\rho}(\xi)\right)$ is monotone on the interval $] 0, \bar{\rho}[$, where $\bar{\rho}:=\min \left\{\operatorname{dist}(x, \partial U),(C \mathbf{A})^{-1}\right\}$.

REMARK 23.3. The proof of Theorem 23.1 can also be given following the alternative argument of Spolaor in [Spo19], which uses the Hardt-Simon inequality and the classical version by Allard of Moser's iteration for subharmonic functions on varifolds. While Spolaor's argument is more flexible and indeed works for integral currents which are not minimizing but sufficiently close to minimizing ones in a suitably quantified way, we prefer to adhere to the strategy of [DLS14] because it is more homogeneous to our notation and terminology.

\section{Proof of Proposition 17.32}

The proof follows the one of [DLS16a, Proposition 3.7] given in [DLS16a, Section 9] with minor modifications. The necessary tools used there, namely the splitting before tilting Propositions, the height bound and the reparametrization theorem are all available from the previous sections.

Part 4. Blow-up and final argument

\section{Intervals of flattening}

Our argument for Theorem 4.3 is by contradiction, and we start therefore fixing a current $T$, a submanifold $\Sigma$, an open set $\Omega$, an integer $2 \leq Q \leq \frac{p}{2}$, positive reals $\alpha$ and $\eta$ and a sequence $r_{k} \downarrow 0$ of radii as in Proposition 8.7. In this section we proceed as in [DLS16b, Section 2] and define appropriate intervals of flattening $\left.] s_{j}, t_{j}\right]$, which are intervals over which we will construct appropriate center manifolds. These intervals, which will be ordered so that $t_{j+1} \leq s_{j}$ will satisfy several properties, among which we anticipate the following fundamental one: aside from finitely many exceptions, each radius $r_{k}$ belongs to one of the intervals. In particular, if they are finitely many, then 0 is the left endpoint of the last one, whereas if they are infinitely many, then $t_{j} \downarrow 0$. The definition of these intervals is taken literally from [DLS16b, Section 2.1], the only difference being that we take advantage of Theorem 17.19 in place of [DLS16a, Theorem 1.17]. However we repeat the details for the reader's convenience.

Without loss of generality we assume that $\mathbf{B}_{6 \sqrt{m}}(0) \subset \Omega$, and we fix a small parameter $\left.\varepsilon_{3} \in\right] 0, \varepsilon_{2}\left[\right.$, where $\varepsilon_{2}$ is the constant appearing in (17.8) of Assumption 17.5. 
Then, we take advantage of Proposition 8.7 and of a simple rescaling argument to assume further that:

$$
\begin{gathered}
T_{0} \Sigma=\mathbb{R}^{m+\bar{n}} \times\{0\}, \quad \Theta(T, 0)=Q, \quad \partial T\left\llcorner\mathbf{B}_{6 \sqrt{m}}(0)=0 \bmod (p),\right. \\
\|T\|\left(\mathbf{B}_{6 \sqrt{m} \rho}(0)\right) \leq\left(Q(6 \sqrt{m})^{m}+\varepsilon_{3}^{2}\right) \rho^{m} \quad \text { for all } \rho \leq 1 \\
\mathbf{c}\left(\Sigma \cap \mathbf{B}_{7 \sqrt{m}}(0)\right) \leq \varepsilon_{3} .
\end{gathered}
$$

We next define

$$
\left.\mathcal{R}:=\{r \in] 0,1]: \mathbf{E}^{n o}\left(T, \mathbf{B}_{6 \sqrt{m} r}(0)\right) \leq \varepsilon_{3}^{2}\right\},
$$

Observe that $\{0\} \cup \mathcal{R}$ is a closed set and that, since $\mathbf{E}^{n o}\left(T, \mathbf{B}_{6 \sqrt{m} r_{k}}\right) \rightarrow 0$ as $k \uparrow \infty$, $r_{k} \in \mathcal{R}$ for $k$ large enough.

The intervals of flattening will form a covering of $\mathcal{R}$. We first define $t_{0}$ as the maximum of $\mathcal{R}$. We then define inductively $s_{0}, \ldots, t_{j}, s_{j}$ in the following way.

Let us first assume that we have defined $t_{j}$ and we wish to define $s_{j}$ (in particular this part is applied also with $j=0$ to define $\left.s_{0}\right)$. We first consider the rescaled current $T_{j}:=\left(\left(\iota_{0, t_{j}}\right)_{\sharp} T\right)\left\llcorner\mathbf{B}_{6 \sqrt{m}}, \Sigma_{j}:=\iota_{0, t_{j}}(\Sigma) \cap \mathbf{B}_{7 \sqrt{m}}\right.$; moreover, consider for each $j$ an orthonormal system of coordinates so that, if we denote by $\pi_{0}$ the $m$-plane $\mathbb{R}^{m} \times\{0\}$, then $\mathbf{E}^{n o}\left(T_{j}, \mathbf{B}_{6 \sqrt{m}}, \pi_{0}\right)=\mathbf{E}^{n o}\left(T_{j}, \mathbf{B}_{6 \sqrt{m}}\right)$ (alternatively we can keep the system of coordinates fixed and rotate the currents $T_{j}$ ).

Definition 25.1. We let $\mathcal{M}_{j}$ be the corresponding center manifold constructed in Theorem 17.19 applied to $T_{j}$ and $\Sigma_{j}$ with respect to the $m$-plane $\pi_{0}$. The manifold $\mathcal{M}_{j}$ is then the graph of a map $\varphi_{j}: \pi_{0} \supset[-4,4]^{m} \rightarrow \pi_{0}^{\perp}$, and we set $\Phi_{j}(x):=$ $\left(x, \varphi_{j}(x)\right) \in \pi_{0} \times \pi_{0}^{\perp}$. We then let $\mathscr{W}^{(j)}$ be the Whitney decomposition of $[-4,4]^{m} \subset$ $\pi_{0}$ as in Definition 17.9, applied to $T_{j}$. We denote by $\mathbf{p}_{j}$ the orthogonal projection on the center manifold $\mathcal{M}_{j}$, which, given the $C^{3, \kappa}$ estimate on $\varphi_{j}$, is well defined in a "slab" $\mathbf{U}_{j}$ of thickness 1 as defined in point (U) of Assumption 17.21.

Next we distinguish two cases:

(Go) For every $L \in \mathscr{W}^{(j)}$,

$$
\ell(L)<c_{s} \operatorname{dist}(0, L),
$$

where $c_{s}:=\frac{1}{64 \sqrt{m}}$, see Proposition 17.32. In this case we set $s_{j}=0$. Observe that in this case the origin is included in the set $\Gamma_{j}$ defined in (17.17).

(Stop) Assuming that (Go) fails, we fix an $L$ with maximal diameter among those cubes of $\mathscr{W}^{(j)}$ which violate the inequality (25.5). We then set

$$
s_{j}:=t_{j} \frac{\ell(L)}{c_{s}} .
$$


Observe that, in both cases, for every $\rho>\bar{r}:=s_{j} / t_{j}$ we have

$$
\ell(L)<c_{s} \rho \quad \text { for all } L \in \mathscr{W}^{(j)} \text { with } L \cap B_{\rho}\left(0, \pi_{0}\right) \neq \emptyset .
$$

We next come to the definition of $t_{j+1}$ once we know $s_{j}$. If $s_{j}=0$, then we stop the procedure and we end up with finitely many intervals of flattening. Otherwise we let $t_{j+1}$ be the maximum of $\left.\left.\mathcal{R} \cap\right] 0, s_{j}\right]$. Note that, since the vanishing sequence $\left\{r_{k}\right\}$ belongs to $\mathcal{R}$ except for finitely many elements, clearly the latter set is nonempty and thus $t_{j+1}$ is a positive number. Observe also that, by (17.18) of Proposition 17.13 and using that $2^{-N_{0}}<c_{s}$ by (17.16), we have $\ell(L) \leq 2^{-6-N_{0}} \leq \frac{c_{s}}{64}$. Thus, $\frac{s_{j}}{t_{j}}<2^{-5}$. This ensures that, in case (Go) never holds (i.e. the intervals of flattening are infinitely many), $t_{j} \downarrow 0$.

Definition 25.2. We denote by $\mathcal{F}$ the (finite or countable) family of intervals of flattening as defined above.

The following proposition is the analog of [DLS16b, Proposition 2.2] and, since the proof is a minor modification of the one given in [DLS16b, Section 2.2] we omit it. Using the notation of Definition 17.12 we introduce the subfamilies $\mathscr{W}_{e}^{(j)}, \mathscr{W}_{h}^{(j)}$ and $\mathscr{W}_{n}^{(j)}$. Recall also that, given two sets $A$ and $B$, we have defined their separation as the number $\operatorname{sep}(A, B):=\inf \{|x-y|: x \in A, y \in B\}$.

Proposition 25.3. Assuming $\varepsilon_{3}$ sufficiently small, then the following holds:

(i) $s_{j}<\frac{t_{j}}{2^{5}}$ and the family $\mathcal{F}$ is either countable and $t_{j} \downarrow 0$, or finite and $\left.\left.I_{j}=\right] 0, t_{j}\right]$ for the largest $j$;

(ii) the union of the intervals of $\mathcal{F}$ cover $\mathcal{R}$, and for $k$ large enough the radii $r_{k}$ in Proposition 8.7 belong to $\mathcal{R}$;

(iii) if $r \in] \frac{s_{j}}{t_{j}}, 3\left[\right.$ and $J \in \mathscr{W}_{n}^{(j)}$ intersects $B:=\mathbf{p}_{\pi_{0}}\left(\mathcal{B}_{r}\left(q_{j}\right)\right)$, with $q_{j}:=\Phi_{j}(0)$, then $J$ is in the domain of influence $\mathscr{W}_{n}^{(j)}(H)$ (see Definition 17.28) of a cube $H \in \mathscr{W}_{e}^{(j)}$ with

$$
\ell(H) \leq 3 c_{s} r \quad \text { and } \quad \max \{\operatorname{sep}(H, B), \operatorname{sep}(H, J)\} \leq 3 \sqrt{m} \ell(H) \leq \frac{3 r}{16} ;
$$

(iv) $\mathbf{E}^{n o}\left(T_{j}, \mathbf{B}_{r}\right) \leq C_{0} \varepsilon_{3}^{2} r^{2-2 \delta_{2}}$ for every $\left.r \in\right] \frac{s_{j}}{t_{j}}, 3[$.

(v) $\sup \left\{\operatorname{dist}\left(x, \mathcal{M}_{j}\right): x \in \operatorname{spt}\left(T_{j}\right) \cap \mathbf{p}_{j}^{-1}\left(\mathcal{B}_{r}\left(q_{j}\right)\right)\right\} \leq C_{0}\left(\boldsymbol{m}_{0}^{j}\right)^{\frac{1}{2 m}} r^{1+\beta_{2}}$ for every $r \in] \frac{s_{j}}{t_{j}}, 3\left[\right.$, where $\boldsymbol{m}_{0}^{j}:=\max \left\{\mathbf{c}\left(\Sigma_{j}\right)^{2}, \mathbf{E}^{n o}\left(T_{j}, \mathbf{B}_{6 \sqrt{m}}\right)\right\}$.

\section{Frequency functions and its variations}

As in [DLS16b, Section 3] we introduce the following Lipschitz (piecewise linear) weight

$$
\phi(r):= \begin{cases}1 & \text { for } r \in\left[0, \frac{1}{2}\right] \\ 2-2 r & \text { for } \left.r \in] \frac{1}{2}, 1\right] \\ 0 & \text { for } r \in] 1,+\infty[\end{cases}
$$


For every interval of flattening $\left.\left.I_{j}=\right] s_{j}, t_{j}\right] \in \mathcal{F}$, we let $N_{j}$ be the normal approximation of $T_{j}$ on the center manifold $\mathcal{M}_{j}$ of Thereom 17.24. As in [DLS16b, Section $3]$ we introduce the corresponding frequency functions and state the main analytical estimate, which allows us to exclude infinite order of contact of the normal approximations with the center manifolds $\mathcal{M}_{j}$.

Definition 26.1 (Frequency functions). For every $r \in] 0,3]$ we define:

$$
\mathbf{D}_{j}(r):=\int_{\mathcal{M}^{j}} \phi\left(\frac{d_{j}(q)}{r}\right)\left|D N_{j}\right|^{2}(q) d q \quad \text { and } \quad \mathbf{H}_{j}(r):=-\int_{\mathcal{M}^{j}} \phi^{\prime}\left(\frac{d_{j}(q)}{r}\right) \frac{\left|N_{j}\right|^{2}(q)}{d(q)} d q,
$$

where $d_{j}(q)$ is the geodesic distance on $\mathcal{M}_{j}$ between $q$ and $\Phi_{j}(0)$, and $d q$ is short for $d \mathcal{H}^{m}(q)$. If $\mathbf{H}_{j}(r)>0$, we define the frequency function $\mathbf{I}_{j}(r):=\frac{r \mathbf{D}_{j}(r)}{\mathbf{H}_{j}(r)}$.

Theorem 26.2 (Main frequency estimate). If $\varepsilon_{3}$ is sufficiently small, then there exists a geometric constant $C_{0}$ such that, for every $[a, b] \subset\left[\frac{s_{j}}{t_{j}}, 3\right]$ with $\left.\mathbf{H}_{j}\right|_{[a, b]}>0$, we have

$$
\mathbf{I}_{j}(a) \leq C_{0}\left(1+\mathbf{I}_{j}(b)\right)
$$

To simplify the notation, in this section we drop the index $j$ and omit the measure $\mathcal{H}^{m}$ in the integrals over regions of $\mathcal{M}$. The proof exploits four identities collected in Proposition 26.4, which is the analog of [DLS16b, Proposition 3.5] and whose proof will be discussed in the next sections. Following [DLS16b, Section 3] we introduce further auxiliary functions in order to express derivatives and estimates on the functions $\mathbf{D}, \mathbf{H}$ and $\mathbf{I}$. We also remind the reader that in principle we must distinguish two situations:

- If $Q<\frac{p}{2}$, then the normal approximations are $\mathcal{A}_{Q}\left(\mathbb{R}^{m+n}\right)$-valued maps and thus all the quantities considered here coincide literally with the ones defined in [DLS16b, Section 3].

- If $Q=\frac{p}{2}$, then the normal approximations take values in $\mathscr{A}_{Q}\left(\mathbb{R}^{m+n}\right)$; in this case we use the notational conventions of [DLHMS, Subsection 7.1] and thus, although at the formal level the definitions of the various objects are identical, the notation is underlying the fact that all integrals involved in the computations must be split into three domains to be reduced to integrals of expressions involving the $\mathcal{A}_{Q}\left(\mathbb{R}^{m+n}\right)$-valued maps $N^{+}, N^{-}$and $Q \llbracket \boldsymbol{\eta} \circ N \rrbracket$.

DeFinition 26.3. We let $\partial_{\hat{r}}$ denote the derivative with respect to arclength along geodesics starting at $\Phi(0)$. We set

$$
\begin{aligned}
\mathbf{E}(r) & :=-\int_{\mathcal{M}} \phi^{\prime}\left(\frac{d(q)}{r}\right) \sum_{i=1}^{Q}\left\langle N_{i}(q), \partial_{\hat{r}} N_{i}(q)\right\rangle d q \\
\mathbf{G}(r) & :=-\int_{\mathcal{M}} \phi^{\prime}\left(\frac{d(q)}{r}\right) d(q)\left|\partial_{\hat{r}} N(q)\right|^{2} d q \text { and } \Sigma(r):=\int_{\mathcal{M}} \phi\left(\frac{d(q)}{r}\right)|N|^{2}(q) d q .
\end{aligned}
$$


As in [DLS16b, Section 3] we observe that the estimate

$$
\left.\mathbf{D}(r) \leq \int_{\mathcal{B}_{r}(\Phi(0))}|D N|^{2} \leq C_{0} \boldsymbol{m}_{0} r^{m+2-2 \delta_{2}} \quad \text { for every } \quad r \in\right] \frac{s}{t}, 3[.
$$

is a consequence of the inequality (17.24) in Theorem 17.24. Consider indeed that (25.7) bounds the side of each Whitney region $\mathcal{L}$ intersecting $\mathcal{B}_{r}(\Phi(0))$ and that, on the contact region $\mathcal{K}$ the map $N$ vanishes identically: it suffices therefore to sum the estimates (17.24) over the aforementioned Whitney regions $\mathcal{L}$.

We are now ready to state the key four identities, cf. [DLS16b, Proposition 3.5]:

Proposition 26.4. (First variation estimates) For every $\gamma_{3}$ sufficiently small there is a constant $C=C\left(\gamma_{3}\right)>0$ such that, if $\varepsilon_{3}$ is sufficiently small, $[a, b] \subset\left[\frac{s}{t}, 3\right]$ and $\mathbf{I} \geq 1$ on $[a, b]$, then the following inequalities hold for a.e. $r \in[a, b]$ :

$$
\begin{aligned}
\left|\mathbf{H}^{\prime}(r)-\frac{m-1}{r} \mathbf{H}(r)-\frac{2}{r} \mathbf{E}(r)\right| & \leq C \mathbf{H}(r) \\
\left|\mathbf{D}(r)-r^{-1} \mathbf{E}(r)\right| & \leq C \mathbf{D}(r)^{1+\gamma_{3}}+C \varepsilon_{3}^{2} \Sigma(r) \\
\left|\mathbf{D}^{\prime}(r)-\frac{m-2}{r} \mathbf{D}(r)-\frac{2}{r^{2}} \mathbf{G}(r)\right| & \leq C \mathbf{D}(r)+C \mathbf{D}(r)^{\gamma_{3}} \mathbf{D}^{\prime}(r)+C r^{-1} \mathbf{D}(r)^{1+\gamma_{3}} \\
\Sigma(r)+r \Sigma^{\prime}(r) & \leq C r^{2} \mathbf{D}(r) \leq C r^{2+m} \varepsilon_{3}^{2}
\end{aligned}
$$

Theorem 26.2 follows from the latter four estimates and from (26.4) through the computations given in [DLS16b, Section 3]. The proofs of the estimates (26.5) and (26.8) given in [DLS16b, Section 3] are valid in our case as well, since they do not exploit the connection between the approximation and the currents, but they are in fact valid for any map $N$ satisfying $\mathbf{I} \geq 1$. We therefore focus on (26.6) and (26.7) which are instead obtained from first variation arguments applied to the area minimizing current $T_{j}$. In our case the current is area minimizing $\bmod (p)$, however a close inspection of the proofs in [DLS16b] shows that the computations in there can be transferred to our case because the varifold induced by $T_{j}$ is stationary (and the required estimates relating the varifold induced by the graph of $N_{j}$ in the normal bundle of $\mathcal{M}_{j}$ and the current $T_{j}$ have been proved in the previous section).

In the rest of the section we omit the subscript $j$ from $T_{j}, \Sigma_{j}, \mathcal{M}_{j}$ and $N_{j}$.

26.1 First variations. We recall the vector field used in [DLS16b]. We will consider:

- the outer variations, where $X(q)=X_{o}(q):=\phi\left(\frac{d(\mathbf{p}(q))}{r}\right)(q-\mathbf{p}(q))$;

- the inner variations, where $X(q)=X_{i}(q):=Y(\mathbf{p}(q))$ with

$$
Y(q):=\frac{d(q)}{r} \phi\left(\frac{d(q)}{r}\right) \frac{\partial}{\partial \hat{r}} \quad \forall q \in \mathcal{M}
$$


Note that $X_{i}$ is the infinitesimal generator of a one parameter family of bilipschitz homeomorphisms $\Phi_{\varepsilon}$ defined as $\Phi_{\varepsilon}(q):=\Psi_{\varepsilon}(\mathbf{p}(q))+q-\mathbf{p}(q)$, where $\Psi_{\varepsilon}$ is the oneparameter family of bilipschitz homeomorphisms of $\mathcal{M}$ generated by $Y$.

Consider now the map $F(q):=\sum_{i} \llbracket q+N_{i}(q) \rrbracket$ and the current $\mathbf{T}_{F}$ associated to its image: in particular we are using the conventions of [DLS15] in the case $Q<\frac{p}{2}$ (i.e. when $N$ takes values in $\mathcal{A}_{Q}\left(\mathbb{R}^{m+n}\right)$ ) and the conventions introduced in [DLHMS, Definition 11.2] in the case $Q=\frac{p}{2}$ (i.e. when $N$ takes values in $\mathscr{A}_{Q}\left(\mathbb{R}^{m+n}\right)$ ). As in [DLS16b, Section 3.3] we observe that, although the vector fields $X=X_{o}$ and $X=X_{i}$ are not compactly supported, it is easy to see that $\delta T(X)=\delta T\left(X^{T}\right)+$ $\delta T\left(X^{\perp}\right)=\delta T\left(X^{\perp}\right)$, where $X=X^{T}+X^{\perp}$ is the decomposition of $X$ in the tangent and normal components to $T \Sigma$.

Then, we have

$$
\begin{aligned}
\left|\delta \mathbf{T}_{F}(X)\right| \leq & \left|\delta \mathbf{T}_{F}(X)-\delta T(X)\right|+\left|\delta T\left(X^{\perp}\right)\right| \\
\leq & \underbrace{\int_{\operatorname{spt}(T) \backslash \operatorname{Im}(F)}\left|\operatorname{div} \vec{T}_{T} X\right| d\|T\|+\int_{\operatorname{Im}(F) \backslash \operatorname{spt}(T)}\left|\operatorname{div} \overrightarrow{\mathbf{T}}_{F} X\right| d\left\|\mathbf{T}_{F}\right\|}_{\operatorname{Err}_{4}} \\
& +\underbrace{\left|\int \operatorname{div}_{\vec{T}} X^{\perp} d\|T\|\right|}_{\operatorname{Err}_{5}} .
\end{aligned}
$$

In order to simplify the notation we set $\varphi_{r}(x):=\phi\left(\frac{d(x)}{r}\right)$. Next, we apply [DLS15, Theorem 4.2] in the case $Q<\frac{p}{2}$ (this corresponds exactly to what done in [DLS16b, Section 3.3] and [DLHMS, Theorem 14.2] when $Q=\frac{p}{2}$ to conclude

$$
\delta \mathbf{T}_{F}\left(X_{o}\right)=\int_{\mathcal{M}}\left(\varphi_{r}|D N|^{2}+\sum_{i=1}^{Q} N_{i} \otimes \nabla \varphi_{r}: D N_{i}\right)+\sum_{j=1}^{3} \operatorname{Err}_{j}^{o},
$$

where the errors $\operatorname{Err}_{j}^{o}$ correspond to the terms $\operatorname{Err}_{j}$ of [DLS15, Theorem 4.2] in case $Q<\frac{p}{2}$ and to the analogous terms in [DLHMS, Theorem 14.2] when $Q=\frac{p}{2}$. This implies

$$
\begin{aligned}
\operatorname{Err}_{1}^{o} & =-Q \int_{\mathcal{M}} \varphi_{r}\left\langle H_{\mathcal{M}}, \boldsymbol{\eta} \circ N\right\rangle \\
\left|\operatorname{Err}_{2}^{o}\right| & \leq C_{0} \int_{\mathcal{M}}\left|\varphi_{r}\right||A|^{2}|N|^{2}, \\
\left|\operatorname{Err}_{3}^{o}\right| & \leq C_{0} \int_{\mathcal{M}}\left(|N||A|+|D N|^{2}\right)\left(\left|\varphi_{r}\right||D N|^{2}+\left|D \varphi_{r}\right||D N||N|\right),
\end{aligned}
$$

where $H_{\mathcal{M}}$ is the mean curvature vector of $\mathcal{M}$. In particular we conclude

$$
\left|\mathbf{D}(r)-r^{-1} \mathbf{E}(r)\right| \leq \sum_{j=1}^{5}\left|\operatorname{Err}_{j}^{o}\right|
$$


where $\operatorname{Err}_{4}^{o}$ and $\operatorname{Err}_{5}^{o}$ denote the terms $\operatorname{Err}_{4}$ and $\operatorname{Err}_{5}$ of (26.9) when $X=X_{o}$.

We follow the same arguments with $X=X_{i}$, applying this time [DLS15, Theorem 4.3] for $Q<\frac{p}{2}$ and [DLHMS, Theorem 14.3] for $Q=\frac{p}{2}$. In particular using the formulas [DLS16b, (3.29)\&(3.30)] for $\operatorname{div}_{\mathcal{M}} Y$ and $D_{\mathcal{M}} Y$ we conclude

$$
\left|\mathbf{D}^{\prime}(r)-(m-2) r^{-1} \mathbf{D}(r)-2 r^{-2} \mathbf{G}(r)\right| \leq C_{0} \mathbf{D}(r)+\sum_{j=1}^{5}\left|\operatorname{Err}_{j}^{i}\right|,
$$

where

$$
\begin{aligned}
\operatorname{Err}_{1}^{i} & =-Q \int_{\mathcal{M}}\left(\left\langle H_{\mathcal{M}}, \boldsymbol{\eta} \circ N\right\rangle \operatorname{div}_{\mathcal{M}} Y+\left\langle D_{Y} H_{\mathcal{M}}, \boldsymbol{\eta} \circ N\right\rangle\right) \\
\left|\operatorname{Err}_{2}^{i}\right| & \leq C_{0} \int_{\mathcal{M}}|A|^{2}\left(|D Y||N|^{2}+|Y||N||D N|\right) \\
\left|\operatorname{Err}_{3}^{i}\right| & \leq C_{0} \int_{\mathcal{M}}\left(|Y||A||D N|^{2}(|N|+|D N|)+|D Y|\left(|A||N|^{2}|D N|+|D N|^{4}\right)\right)
\end{aligned}
$$

and where $\operatorname{Err}_{4}^{i}$ and $\operatorname{Err}_{5}^{i}$ denote the terms $\operatorname{Err}_{4}$ and $\operatorname{Err}_{5}$ of (26.9) when $X=X_{i}$.

26.2 Error estimates. We next proceed as in [DLS16b, Section 4]. First of all, since the structure and estimates on the size of the cubes of the Whitney decomposition are exactly the same, we can define the regions of [DLS16b, Section 4.1] and deduce the same conclusions of [DLS16b, Lemma 4.4]. Next, since our estimates in Theorem 17.24 have the same structure of [DLS16a, Theorem 2.4], we conclude the validity of all the estimates in [DLS16b, Section 4.2]. In turn we can repeat all the arguments in [DLS16b, Section 4.3] to conclude the same estimates for the terms of type $\operatorname{Err}_{1}^{o}, \operatorname{Err}_{1}^{i}, \operatorname{Err}_{2}^{o}, \operatorname{Err}_{2}^{i}, \operatorname{Err}_{3}^{o}, \operatorname{Err}_{3}^{i}, \operatorname{Err}_{4}^{o}, \operatorname{Err}_{4}^{i}, \operatorname{Err}_{5}^{o}$. Some more care is needed to handle the term $\operatorname{Err}_{5}^{i}$. First of all we split the latter error into the terms $I_{1}$ and $I_{2}$ of [DLS16b, Page 596]. The term $I_{1}$ is estimated in the same way. Fo $\mathrm{r}$ the term $I_{2}$ we can use the same argument when $Q<\frac{p}{2}$ and hence $F$ is $\mathcal{A}_{Q}$-valued. However, we need a small modification in the case $Q=\frac{p}{2}$, when $F$ is $\mathscr{A}_{Q}$-valued.

As in [DLS16b, Page 597] we start by introducing an orthonormal frame $\nu_{1}, \ldots, \nu_{l}$ for $T_{q} \Sigma^{\perp}$ of class $C^{2, \varepsilon_{0}}$ (cf. [DLS15, Appendix A]) and set

$$
h_{q}^{j}(\vec{\lambda}):=-\sum_{k=1}^{m}\left\langle D_{v_{k}} \nu_{j}(q), v_{k}\right\rangle
$$

whenever $v_{1} \wedge \ldots \wedge v_{m}=\vec{\lambda}$ is an $m$-vector of $T_{q} \Sigma$, with $v_{1}, \ldots, v_{m}$ orthonormal.

Next, we recall the canonical decomposition of $\mathcal{M}$ into $\mathcal{M}_{+}, \mathcal{M}_{-}$and $\mathcal{M}_{0}$ induced by $F$ (see Section 2) and correspondingly, we decompose the image of $F$ into

$$
\begin{aligned}
\operatorname{Im}_{0}(F) & :=\operatorname{Im}(F) \cap \mathbf{p}^{-1}\left(\mathcal{M}_{0}\right), \\
\operatorname{Im}_{+}(F) & :=\operatorname{Im}(F) \cap \mathbf{p}^{-1}\left(\mathcal{M}_{+}\right), \\
\operatorname{Im}_{-}(F) & :=\operatorname{Im}(F) \cap \mathbf{p}^{-1}\left(\mathcal{M}_{-}\right) .
\end{aligned}
$$


If $q \in \operatorname{Im}(F)$, as in [DLS16b, Page 597] we set

$$
h_{\mathbf{p}(q)}^{j}:=h_{\mathbf{p}(q)}^{j}(\overrightarrow{\mathcal{M}}(\mathbf{p}(q))) \text { and } \quad h_{\mathbf{p}(q)}=\sum_{j=1}^{l} h_{\mathbf{p}(q)}^{j} \nu_{j}(\mathbf{p}(q)) .
$$

If $q \in \operatorname{Im}_{0}(F) \cup \operatorname{Im}_{+}(F)$, as in [DLS16b, Page 597] we set

$$
h_{q}^{j}:=h_{q}^{j}\left(\overrightarrow{\mathbf{T}}_{F}(q)\right) \quad \text { and } \quad h_{q}=\sum_{j=1}^{l} h_{q}^{j} \nu_{j}(q)
$$

We proceed however differently for $q \in \operatorname{Im}_{-}(F)$ : in this case we set

$$
h_{q}^{j}:=h_{q}^{j}\left(-\overrightarrow{\mathbf{T}}_{F}(q)\right) \quad \text { and } \quad h_{q}=\sum_{j=1}^{l} h_{q}^{j} \nu_{j}(q) .
$$

Observe that, since for $q \in \operatorname{Im}_{-}(F)$ we have $-\overrightarrow{\mathbf{T}}_{F}(q)=\overrightarrow{\mathbf{T}}_{F^{-}}(q)$, in practice we can follow precisely the same computations of [DLS16b, Page 597] in each of the regions $\operatorname{Im}_{0}(F), \operatorname{Im}_{+}(F)$ and $\operatorname{Im}_{-}(F)$, to conclude

$$
\begin{aligned}
\left\langle X_{i}(q), h_{q}\right\rangle= & \left\langle X_{i}(q),\left(h_{q}-h_{\mathbf{p}(q)}\right)\right\rangle=\sum_{j}\left\langle X_{i}(\mathbf{p}(q)), D \nu_{j}(\mathbf{p}(q)) \cdot \mathbf{e x}_{\mathbf{p}(q)}^{-1}(q)\right\rangle h_{\mathbf{p}(q)}^{j} \\
& +\sum_{j}\left\langle\nu_{j}(q), X_{i}(q)\right\rangle\left(h_{q}^{j}-h_{\mathbf{p}(q)}^{j}\right)+O\left(|q-\mathbf{p}(q)|^{2}\right) \\
= & \sum_{j}\left\langle X_{i}(\mathbf{p}(q)), D \nu_{j}(\mathbf{p}(q)) \cdot \mathbf{e x}_{\mathbf{p}(q)}^{-1}(q)\right\rangle h_{\mathbf{p}(q)}^{j} \\
& +O\left(\left|\overrightarrow{\mathbf{T}}_{F}(q)-\overrightarrow{\mathcal{M}}(\mathbf{p}(q))\right|_{n o}|q-\mathbf{p}(q)|+|q-\mathbf{p}(q)|^{2}\right)
\end{aligned}
$$

Observe that the only difference with [DLS16b, (4.17)] is that $\left|\overrightarrow{\mathbf{T}}_{F}(q)-\overrightarrow{\mathcal{M}}(\mathbf{p}(q))\right|_{\text {no }}$ replaces $\left|\overrightarrow{\mathbf{T}}_{F}(q)-\overrightarrow{\mathcal{M}}(\mathbf{p}(q))\right|$ in the last line of the above estimate. Next, for $q \in$ $\operatorname{spt}\left(\mathbf{T}_{F}\right)$, we can bound $|q-\mathbf{p}(q)| \leq|N(q)|$ and $\left|\overrightarrow{\mathbf{T}}_{F}(q)-\overrightarrow{\mathcal{M}}(\mathbf{p}(q))\right|_{n o} \leq C|D N(\mathbf{p}(q))|$. We therefore conclude the estimate

$$
\left\langle X_{i}(q), h_{q}\right\rangle=\sum_{j}\left\langle X_{i}(\mathbf{p}(q)), D \nu_{j}(\mathbf{p}(q)) \cdot \mathbf{e x}_{\mathbf{p}(q)}^{-1}(q)\right\rangle h_{\mathbf{p}(q)}^{j}+O\left(|N|^{2}(\mathbf{p}(q))+|D N|^{2}(\mathbf{p}(q))\right) .
$$

Combining the latter inequality with [DLHMS, Theorem 13.1] we can bound

$$
\begin{aligned}
& I_{2}^{i}=\left|\int\left\langle X_{i}, h_{q}\right\rangle d\left\|\mathbf{T}_{F}\right\|\right|=\left|\sum_{i=1}^{Q} \int_{\mathcal{M}}\left\langle Y, h_{F_{i}}\right\rangle \mathbf{J} F_{i}\right| \\
& \stackrel{(26.22)}{\leq}\left|\int_{\mathcal{M}} \sum_{j=1}^{l} \sum_{i=1}^{Q}\left\langle Y(x), D \nu_{j}(x) \cdot \mathbf{e x}_{x}^{-1}\left(F_{i}(x)\right)\right\rangle h_{x}^{j} d \mathcal{H}^{m}(x)\right|+C \int_{\mathcal{M}} \varphi_{r}\left(|N|^{2}+|D N|^{2}\right) .
\end{aligned}
$$

We can now proceed as in [DLS16b, Page 598] to conclude the same estimate for $I_{2}$. 


\section{Boundedness of the frequency function and reverse Sobolev}

We next show the counterpart of [DLS16b, Theorem 5.1].

Theorem 27.1 (Boundedness of the frequency functions). Let $T$ be as in Proposition 8.7. If the intervals of flattening are $j_{0}<\infty$, then there is $\rho>0$ such that

$$
\left.\mathbf{H}_{j_{0}}>0 \text { on }\right] 0, \rho\left[\text { and } \quad \limsup _{r \rightarrow 0} \mathbf{I}_{j_{0}}(r)<\infty .\right.
$$

If the intervals of flattening are infinitely many, then there is a number $j_{0} \in \mathbb{N}$ and a geometric constant $j_{1} \in \mathbb{N}$ such that

$$
\begin{aligned}
& \left.\mathbf{H}_{j}>0 \text { on }\right] \frac{s_{j}}{t_{j}}, 2^{-j_{1}} 3\left[\text { for all } j \geq j_{0}, \quad \sup _{j \geq j_{0}} \sup _{r \in]} \mathbf{s u p}_{\frac{s_{j}}{t_{j}}, 2^{-j_{1}} 3[} \mathbf{I}_{j}(r)<\infty,\right. \\
& \sup \left\{\min \left\{\mathbf{I}_{j}(r), \frac{r^{2} \int_{\mathcal{B}_{r}}\left|D N_{j}\right|^{2}}{\int_{\mathcal{B}_{r}}\left|N_{j}\right|^{2}}\right\}: j \geq j_{0} \text { and } \max \left\{\frac{s_{j}}{t_{j}}, \frac{3}{2^{j_{1}}}\right\} \leq r<3\right\}<\infty
\end{aligned}
$$

(in the latter inequality we understand $\mathbf{I}_{j}(r)=\infty$ when $\mathbf{H}_{j}(r)=0$ ).

Proof In the first case we can appeal to the same argument as in [DLS16b, Page 599]. In the second case we also proceed as in [DLS16b, Page 599] and partition the extrema $t_{j}$ of the intervals of flattening into two subsets: the class (A) formed by those $t_{j}$ such that $t_{j}=s_{j-1}$ and the complementary class (B). As in [DLS16b, Page 599] we can assume that $j$ is large enough. In the first case we proceed as in [DLS16b, Page 599] where we substitute [DLS16a, Proposition 3.7] with Proposition 17.32. In case $(\mathrm{B})$ by construction there is $\left.\eta_{j} \in\right] 0,1\left[\right.$ such that $\mathbf{E}^{n o}\left(\left(\iota_{0, t_{j}}\right)_{\sharp} T, \mathbf{B}_{6 \sqrt{m}\left(1+\eta_{j}\right)}\right)>\varepsilon_{3}^{2}$. Up to extraction of a subsequence, we can assume that $T_{j}=\left(\iota_{0, t_{j}}\right)_{\sharp} T$ converges to a cone $S$ : the convergence is strong enough to conclude that the excess of the cone is the limit of the excesses of the sequence. Moreover (since $S$ is a cone), the excess $\mathbf{E}^{n o}\left(S, \mathbf{B}_{r}\right)$ is independent of $r$. We then conclude

$$
\varepsilon_{3}^{2} \leq \liminf _{j \rightarrow \infty, j \in(B)} \mathbf{E}^{n o}\left(T_{j}, \mathbf{B}_{3}\right)
$$

We then argue as in [DLS16b, Page 601] using Lemma 27.2 below in place of [DLS16b, Lemma 5.2].

LEMMA 27.2 Assume the intervals of flattening are infinitely many and $\left.r_{j} \in\right] \frac{s_{j}}{t_{j}}, 3[$ is a subsequence (not relabeled) with $\lim _{j}\left\|N_{j}\right\|_{L^{2}\left(\mathcal{B}_{r_{j}} \backslash \mathcal{B}_{r_{j} / 2}\right)}=0$. If $\varepsilon_{3}$ is sufficiently small, then, $\mathbf{E}^{\text {no }}\left(T_{j}, \mathbf{B}_{r_{j}}\right) \rightarrow 0$.

Proof The argument is a modification of that of [DLS16b, Lemma 5.2], which we include for the reader's convenience. First of all note that, by Proposition 25.3, 
$\mathbf{E}^{n o}\left(T_{j}, \mathbf{B}_{r_{j}}\right) \rightarrow 0$ if $r_{j} \rightarrow 0$. Hence, passing to a subsequence, we can assume the existence of a $c>0$ such that

$$
r_{j} \geq c \quad \text { and } \quad \mathbf{E}^{n o}\left(T_{j}, \mathbf{B}_{6 \sqrt{m}}\right) \geq c .
$$

After the extraction of a further subsequence, we can assume the existence of $r$ such that

$$
\int_{\mathcal{B}_{r} \backslash \mathcal{B}_{\frac{3 r}{4}}}\left|N_{j}\right|^{2} \rightarrow 0
$$

and the existence of a $\bmod (p)$ area-minimizing cone $S$ such that $\left(\iota_{0, t_{j}}\right)_{\sharp} T \rightarrow S$. Recall that $S$ is a representative $\bmod (p)$. By (27.4), the cone $S$ cannot be an integer multiple of an $m$-dimensional plane.

We argue as in [DLS16b, Pages 601-602] and conclude that, if $\mathcal{M}$ is the limit of a subsequence (not relabeled) of the $\mathcal{M}_{j}$, then there are two radii $0<s<t$ such that $\operatorname{spt}(S) \cap \mathbf{B}_{t}(0) \backslash \mathbf{B}_{s}(0) \subset \mathcal{M}$. In particular, by the Constancy Theorem $\bmod (p)$ we conclude that $S\left\llcorner\mathbf{B}_{t}(0) \backslash \mathbf{B}_{s}(0)=Q_{0} \llbracket \mathcal{M} \cap \mathbf{B}_{t}(0) \backslash \mathbf{B}_{s}(0) \rrbracket \bmod (p)\right.$ for an integer $Q_{0}$ with $\left|Q_{0}\right| \leq \frac{p}{2}$. Since $S$ is a cone and a representative $\bmod (p)$ we can in fact infer that $S\left\llcorner\mathbf{B}_{t}(0)=Q_{0} \llbracket 0 \rrbracket \nVdash \llbracket \mathcal{M} \cap \partial \mathbf{B}_{t}(0) \rrbracket \bmod (p)\right.$ (in fact it can be easily inferred from the argument in [DLS16b, Pages 601-602] that $Q_{0}=Q$, although this is not needed in our argument). Since $\llbracket 0 \rrbracket \nVdash \llbracket \mathcal{M} \cap \partial \mathbf{B}_{t}(0) \rrbracket$ induces a stationary varifold and $\mathcal{M}$ is the graph of a function with small $C^{3, \varepsilon_{0}}$ norm, we can applied Allard's Theorem to conclude that in fact $\llbracket 0 \rrbracket \nVdash \llbracket \mathcal{M} \cap \partial \mathbf{B}_{t}(0) \rrbracket$ is smooth. This implies that the latter is in fact $\llbracket \pi \cap \mathbf{B}_{t}(0) \rrbracket$ for some $m$-dimensional plane $\pi$, contradicting the fact that $S$ is not a flat cone.

Finally, Theorem 27.1 can be used as in [DLS16b, Section 5] to show [DLS16b, Corollary 5.3], which we restate in our context for the reader's convenience.

Corollary 27.3 (Reverse Sobolev) Let $T$ be as in Proposition 8.7. Then, there exists a constant $C>0$ which depends on $T$ but not on $j$ such that, for every $j$ and for every $\left.r \in] \frac{s_{j}}{t_{j}}, 1\right]$, there is $\left.\left.\sigma \in\right] \frac{3}{2} r, 3 r\right]$ such that

$$
\int_{\mathcal{B}_{\sigma}\left(\Phi_{j}(0)\right)}\left|D N_{j}\right|^{2} \leq \frac{C}{r^{2}} \int_{\mathcal{B}_{\sigma}\left(\Phi_{j}(0)\right)}\left|N_{j}\right|^{2} .
$$

\section{Final contradiction argument}

In this section we complete the proof of Theorem 1.4 showing that, by Proposition 8.7, under the assumption that the theorem is false, we get a contradiction. In particular fix $T, \Sigma, \Omega$ and $r_{k}$ as in Proposition 8.7. We have already remarked that for each $k$ there is an interval of flattening $\left.\left.I_{j(k)}=\right] s_{j(k)}, t_{j(k)}\right]$ containing $r_{k}$. We proceed as in [DLS16b, Section 6] and introduce the following new objects:

- We first apply Corollary 27.3 to $r=\frac{r_{k}}{t_{j(k)}}$ and set $\bar{s}_{k}:=t_{j(k)} \sigma_{k}$, so that $\frac{\bar{s}_{k}}{t_{j(k)}} \in$ ]$\frac{3}{2} \frac{r_{k}}{t_{j(k)}}, 3 \frac{r_{k}}{t_{j(k)}}[$. 
- We set $\bar{r}_{k}:=\frac{2 \bar{s}_{k}}{3 t_{j(k)}}$.

- We rescale our geometric objects, namely

(U1) The currents $\bar{T}_{k}$, the manifolds $\bar{\Sigma}_{k}$ and the center manifolds $\overline{\mathcal{M}}_{k}$ are given respectively by

$$
\begin{gathered}
\bar{T}_{k}=\left(\iota_{0, \bar{r}_{k}}\right)_{\sharp} T_{j(k)}=\left(\left(\iota_{0, \bar{r}_{k} t_{j(k)}}\right)_{\sharp} T\right)\left\llcorner\mathbf{B}_{6 \sqrt{m} / \bar{r}_{k}},\right. \\
\bar{\Sigma}_{k}=\iota_{0, \bar{r}_{k}}\left(\Sigma_{j(k)}\right)=\iota_{0, \bar{r}_{k} t_{j(k)}}(\Sigma), \\
\overline{\mathcal{M}}_{k}=\iota_{0, \bar{r}_{k}}\left(\mathcal{M}_{j(k)}\right) .
\end{gathered}
$$

(U2) In order to define the rescaled maps $\bar{N}_{k}$ on $\overline{\mathcal{M}}_{k}$ we need to distinguish two cases. When $Q<\frac{p}{2}$, the map $\bar{N}_{k}$ takes values in $\mathcal{A}_{Q}\left(\mathbb{R}^{m+n}\right)$ and is defined by

$$
\bar{N}_{k}(q)=\sum_{i=1}^{Q} \llbracket r^{-1}\left(N_{j(k)}\right)_{i}(r q) \rrbracket .
$$

In the case $Q=\frac{p}{2}$, the map $\bar{N}_{k}$ takes values in $\mathscr{A}_{Q}\left(\mathbb{R}^{m+n}\right)$ and is defined analogously. The reader might either use the decomposition of $\mathcal{M}_{j(k)}$ into $\left(\mathcal{M}_{j(k)}\right)_{+},\left(\mathcal{M}_{j(k)}\right)_{-}$and $\left(\mathcal{M}_{j(k)}\right)_{0}$ or, using the original notation in [DLHMS, Definition 2.2],

$$
\bar{N}_{k}(q)=\left(\sum_{i=1}^{Q} \llbracket r^{-1}\left(N_{j(k)}\right)_{i}(r q) \rrbracket, \varepsilon(r q)\right),
$$

where

$$
N_{j(k)}(\tilde{q})=\left(\sum_{i=1}^{Q} \llbracket\left(N_{j(k)}\right)_{i}(\tilde{q}) \rrbracket, \varepsilon(\tilde{q})\right)
$$

and $\varepsilon(\cdot) \in\{-1,1\}$.

Without loss of generality we can assume that $T_{0} \Sigma=\mathbb{R}^{m+\bar{n}} \times\{0\}$, thus the ambient manifolds $\bar{\Sigma}_{k}$ converge to $\mathbb{R}^{m+\bar{n}} \times\{0\}$ locally in $C^{3, \varepsilon_{0}}$. Observe in addition that $\frac{1}{2}<\frac{r_{k}}{\bar{r}_{k} t_{j(k)}}<1$ and hence it follows from Proposition 8.7(ii) that

$$
\mathbf{E}^{n o}\left(\bar{T}_{k}, \mathbf{B}_{\frac{1}{2}}\right) \leq C \mathbf{E}^{n o}\left(T, \mathbf{B}_{r_{k}}\right) \rightarrow 0 .
$$

Indeed Proposition 8.7(ii) implies that $\bar{T}_{k}$ converge to $Q \llbracket \pi_{0} \rrbracket$ both in the sense of varifolds and in the sense of currents $\bmod (p)$. Finally, we recall that, by Proposition 8.7(iii)\&(iii)s,

$$
\begin{array}{lr}
\mathcal{H}_{\infty}^{m-2+\alpha}\left(\mathrm{D}_{Q}\left(\bar{T}_{k}\right) \cap \mathbf{B}_{1}\right) \geq C_{0} r_{k}^{-(m-2+\alpha)} \mathcal{H}_{\infty}^{m-2+\alpha}\left(\mathrm{D}_{Q}(T) \cap \mathbf{B}_{r_{k}}\right) \geq \eta>0 & \text { when } Q<\frac{p}{2}, \\
\mathcal{H}_{\infty}^{m-1+\alpha}\left(\mathrm{D}_{Q}\left(\bar{T}_{k}\right) \cap \mathbf{B}_{1}\right) \geq C_{0} r_{k}^{-(m-1+\alpha)} \mathcal{H}_{\infty}^{m-1+\alpha}\left(\mathrm{D}_{Q}(T) \cap \mathbf{B}_{r_{k}}\right) \geq \eta>0 & \text { when } Q=\frac{p}{2},
\end{array}
$$


where $\alpha$ is a positive number and $C_{0}$ a geometric constant.

As in [DLS16b, Section 6] we claim the counterpart of [DLS16b, Lemma 6.1], namely Lemma 28.1, which implies that $\overline{\mathcal{M}}_{k}$ converge locally to the flat $m$-plane $\pi_{0}$. We also introduce the exponential maps ex $: B_{3} \subset \mathbb{R}^{m} \simeq T_{\bar{q}_{k}} \overline{\mathcal{M}}_{k} \rightarrow \overline{\mathcal{M}}_{k}$ at $\bar{q}_{k}=$ $\Phi_{j(k)}(0) / \bar{r}_{k}$ ( here and in what follows we assume, w.l.o.g., to have applied a suitable rotation to each $\bar{T}_{k}$ so that the tangent plane $T_{\bar{q}_{k}} \overline{\mathcal{M}}_{k}$ coincides with $\left.\mathbb{R}^{m} \times\{0\}\right)$. We are finally ready to define the blow-up maps $N_{k}^{b}: B_{3} \subset \mathbb{R}^{m} \rightarrow \mathcal{A}_{Q}\left(\mathbb{R}^{m+n}\right)$, when $Q<\frac{p}{2}$ and $N_{k}^{b}: B_{3} \subset \mathbb{R}^{m} \rightarrow \mathscr{A}_{Q}\left(\mathbb{R}^{m+n}\right)$, when $Q=\frac{p}{2}$ :

$$
N_{k}^{b}(x):=\mathbf{h}_{k}^{-1} \bar{N}_{k}\left(\mathbf{e x}_{k}(x)\right)
$$

where $\mathbf{h}_{k}:=\left\|\bar{N}_{k}\right\|_{L^{2}\left(\mathcal{B}_{\frac{3}{2}}\right)}$.

Lemma 28.1 (Vanishing lemma). Let $\bar{T}_{k}, \bar{r}_{k}, \overline{\mathcal{M}}_{k}$ and $\bar{\Sigma}_{k}$ be as above. We then have:

(i) $\min \left\{\boldsymbol{m}_{0}^{j(k)}, \bar{r}_{k}\right\} \rightarrow 0$;

(ii) the rescaled center manifolds $\overline{\mathcal{M}}_{k}$ converge (up to subsequences) to $\pi_{0}=\mathbb{R}^{m} \times$ $\{0\}$ in $C^{3, \kappa / 2}\left(\mathbf{B}_{4}\right)$ and the maps $\mathbf{e x}_{k}$ converge in $C^{2, \kappa / 2}$ to the identity map id : $B_{3} \rightarrow B_{3}$;

(iii) there exists a constant $C>0$, depending only on $T$, such that, for every $k$,

$$
\frac{1}{\mathbf{h}_{k}^{2}} \int_{\mathcal{B}_{\frac{3}{2}}}\left|D \bar{N}_{k}\right|^{2} \leq C \int_{B_{\frac{3}{2}}}\left|D N_{k}^{b}\right|^{2} \leq C .
$$

Proof The argument for (i) can be taken from [DLS16b, Proof of Lemma 6.1]. As for part (ii) the argument given in [DLS16b, Section 6] for the convergence of the center manifolds can be shortened considerably observing that it is a direct consequence of Proposition 25.3(v) and the convergence of the currents $\bar{T}_{k}$. The $C^{2, \kappa / 2}$ convergence of the exponential maps follow then immediately from [DLS16b, Proposition A.4]. Finally, (iii) is an obvious consequence of Corollary 27.3.

Having defined the blow-up maps, the final contradiction comes from the following statements.

Theorem 28.2 (Final blow-up). Up to subsequences, the maps $N_{k}^{b}$ converge strongly in $L^{2}\left(B_{\frac{3}{2}}\right)$ to:

- a function $N_{\infty}^{b}: B_{\frac{3}{2}} \rightarrow \mathcal{A}_{Q}\left(\{0\} \times \mathbb{R}^{\bar{n}} \times\{0\}\right)$ when $Q<\frac{p}{2}$;

- a function $N_{\infty}^{b}: B_{\frac{3}{2}} \rightarrow \mathscr{A}_{Q}\left(\{0\} \times \mathbb{R}^{\bar{n}} \times\{0\}\right)$ when $Q=\frac{p}{2}$.

Such limit is Dir-minimizing in $B_{t}$ for every $\left.t \in\right] \frac{5}{4}, \frac{3}{2}$ [ and satisfies $\left\|N_{\infty}^{b}\right\|_{L^{2}\left(B_{\frac{3}{2}}\right)}=1$ and $\boldsymbol{\eta} \circ N_{\infty}^{b} \equiv 0$.

Theorem 28.3 (Large singular set). Let $N_{\infty}^{b}$ be the map of Theorem 28.2 and define

$$
\Upsilon:=\left\{x \in \bar{B}_{1}: N_{\infty}^{b}(x)=Q \llbracket 0 \rrbracket\right\}
$$


Then

$$
\begin{array}{ll}
\mathcal{H}_{\infty}^{m-2+\alpha}(\Upsilon) \geq \frac{\eta}{2} & \text { if } Q<\frac{p}{2}, \\
\mathcal{H}_{\infty}^{m-1+\alpha}(\Upsilon) \geq \frac{\eta}{2} & \text { if } Q=\frac{p}{2},
\end{array}
$$

where $\alpha$ and $\eta$ are the positive constants in (28.4), resp. (28.5).

The two theorems would contradict [DLS11, Theorem 0.11] in case $Q<\frac{p}{2}$ since, arguing as in [DLS16b, Section 6] we easily conclude that $\Upsilon$ is a subset of the singularities of $N_{\infty}^{b}$. In the case $Q=\frac{p}{2}$ we infer instead from [DLHMS, Proposition $10.3]$ that $N_{b}^{\infty}=Q \llbracket \boldsymbol{\eta} \circ N_{b}^{\infty} \rrbracket$ on the whole $B_{3 / 2}$, which in turn would imply $N_{b}^{\infty}=$ $Q \llbracket 0 \rrbracket$. This however contradicts $\left\|N_{b}^{\infty}\right\|_{L^{2}\left(B_{3 / 2}\right)}=1$.

28.1 Proof of Theorem 28.2 Without loss of generality we may assume that $\bar{q}_{k}:=\bar{r}_{k}^{-1} \Phi_{j(k)}(0)$ coincide all with the origin. We then define a new map $\bar{F}_{k}$ on the geodesic ball $\mathcal{B}_{3 / 2} \subset \overline{\mathcal{M}}_{k}$ distinguishing, as usual, the two cases $Q<\frac{p}{2}$ and $Q=\frac{p}{2}$. In the first case we follow the definition of [DLS16b, Section 7.1], namely we set

$$
\bar{F}_{k}(x):=\sum_{i} \llbracket x+\left(\bar{N}_{k}\right)_{i}(x) \rrbracket .
$$

In the case $Q=\frac{p}{2}$ the map $\bar{F}_{k}$ takes values in $\mathscr{A}_{Q}\left(\mathbb{R}^{m+n}\right)$ and it is induced by $\bar{N}_{k}$ in the sense explained at point (N) of [DLHMS, Assumption 11.1]. The argument given in [DLS16b, Section 7.1] works in our case as well and implies the following estimates (where $\gamma$ is some positive exponent independent of $k$ )

$$
\begin{gathered}
\operatorname{Lip}\left(\left.\bar{N}_{k}\right|_{\mathcal{B}_{3 / 2}}\right) \leq C \mathbf{h}_{k}^{\gamma} \quad \text { and } \quad\left\|\bar{N}_{k}\right\|_{C^{0}\left(\mathcal{B}_{3 / 2}\right)} \leq C\left(\boldsymbol{m}_{0}^{j(k)} \bar{r}_{k}\right)^{\gamma}, \\
\mathbf{M}^{p}\left(( \mathbf { T } _ { \overline { F } _ { k } } - \overline { T } _ { k } ) \left\llcorner\left(\mathbf{p}_{k}^{-1}\left(\mathcal{B}_{\frac{3}{2}}\right)\right) \leq C \mathbf{h}_{k}^{2+2 \gamma},\right.\right. \\
\int_{\mathcal{B}_{\frac{3}{2}}}\left|\boldsymbol{\eta} \circ \bar{N}_{k}\right| \leq C \mathbf{h}_{k}^{2} .
\end{gathered}
$$

From these estimates we conclude the strong $L^{2}$ converge of (a subsequence of) $N_{k}^{b}$ to a map $N_{\infty}^{b}$ on $B_{3 / 2}$ taking values, respectively, on $\mathcal{A}_{Q}\left(\{0\} \times \mathbb{R}^{\bar{n}} \times\{0\}\right)\left(\right.$ when $\left.Q<\frac{p}{2}\right)$ and $\mathscr{A}_{Q}\left(\{0\} \times \mathbb{R}^{\bar{n}} \times\{0\}\right)$ (when $\left.Q=\frac{p}{2}\right)$. Moreover it is obvious that $\left\|N_{\infty}^{b}\right\|_{L^{2}\left(B_{3 / 2}\right)}=1$ and that $\boldsymbol{\eta} \circ N_{\infty}^{b} \equiv 0$. Therefore we are only left with proving that $N_{\infty}^{b}$ is Dirminimizing.

Proceeding as in the [DLS16b, Section 7] we assume, without loss of generality, that the Dirichlet energy of $N_{\infty}^{b}$ is nontrivial (otherwise there is nothing to prove). Thus we can assume that that there exists $c_{0}>0$ such that

$$
c_{0} \mathbf{h}_{k}^{2} \leq \int_{\mathcal{B}_{\frac{3}{2}}}\left|D \bar{N}_{k}\right|^{2} .
$$


We proceed as in [DLS16b, Section $7.2 \&$ Section 7.3]: if there is a radius $t \in] \frac{5}{4}, \frac{3}{2}[$ and a function $f$ on $B_{\frac{3}{2}}$ (taking values in $\mathcal{A}_{Q}\left(\mathbb{R}^{\bar{n}}\right)$ when $Q<\frac{p}{2}$, or in $\mathscr{A}_{Q}\left(\mathbb{R}^{\bar{n}}\right.$ ) when $\left.Q=\frac{p}{2}\right)$ such that

$$
\left.f\right|_{B_{\frac{3}{2}} \backslash B_{t}}=\left.N_{\infty}^{b}\right|_{B_{\frac{3}{2}} \backslash B_{t}} \quad \text { and } \quad \operatorname{Dir}\left(f, B_{t}\right) \leq \operatorname{Dir}\left(N_{\infty}^{b}, B_{t}\right)-2 \delta
$$

for some $\delta>0$, we then produce competitors $\tilde{N}_{k}$ for the maps $\bar{N}_{k}$ satisfying

$$
\begin{gathered}
\tilde{N}_{k} \equiv \bar{N}_{k} \quad \text { in } \mathcal{B}_{\frac{3}{2}} \backslash \mathcal{B}_{t}, \quad \operatorname{Lip}\left(\tilde{N}_{k}\right) \leq C \mathbf{h}_{k}^{\gamma}, \quad\left|\tilde{N}_{k}\right| \leq C\left(\boldsymbol{m}_{0}^{k} \bar{r}_{k}\right)^{\gamma}, \\
\int_{\mathcal{B}_{\frac{3}{2}}}\left|\boldsymbol{\eta} \circ \tilde{N}_{k}\right| \leq C \mathbf{h}_{k}^{2} \quad \text { and } \quad \int_{\mathcal{B}_{\frac{3}{2}}}\left|D \tilde{N}_{k}\right|^{2} \leq \int_{\mathcal{B}_{\frac{3}{2}}}\left|D \bar{N}_{k}\right|^{2}-\delta \mathbf{h}_{k}^{2} .
\end{gathered}
$$

Indeed the construction of the maps in [DLS16b, Section 7.2 \& Section 7.3] uses the left composition of $\mathcal{A}_{Q}$-valued maps with classical maps in the sense of [DLS11, Section 1.3.1], which in the $\mathscr{A}_{Q}$-valued case is substituted by the left composition as defined in [DLHMS, Subsection 7.3].

Consider next the map $\tilde{F}_{k}$ given by $\tilde{F}_{k}(x)=\sum_{i} \llbracket x+\left(\tilde{N}_{k}\right)_{i}(x) \rrbracket$ in the case $Q<\frac{p}{2}$ and by the corresponding $\left(\sum_{i} \llbracket x+\left(\tilde{N}_{k}\right)_{i}(x) \rrbracket, \varepsilon(x)\right)$ in the case $Q=\frac{p}{2}$. The current $\mathbf{T}_{\tilde{F}_{k}}$ coincides with $\mathbf{T}_{\bar{F}_{k}}$ on $\mathbf{p}_{k}^{-1}\left(\mathcal{B}_{\frac{3}{2}} \backslash \mathcal{B}_{t}\right)$. Define the function $\varphi_{k}(q)=\operatorname{dist}_{\overline{\mathcal{M}}_{k}}\left(0, \mathbf{p}_{k}(q)\right)$ and consider for each $s \in] t, \frac{3}{2}\left[\right.$ the slices $\left\langle\mathbf{T}_{\tilde{F}_{k}}-\bar{T}_{k}, \varphi_{k}, s\right\rangle$. By (28.11) we have

$$
\int_{t}^{\frac{3}{2}} \mathbf{M}^{p}\left(\left\langle\mathbf{T}_{\tilde{F}_{k}}-\bar{T}_{k}, \varphi_{k}, s\right\rangle\right) \leq C \mathbf{h}_{k}^{2+\gamma}
$$

Thus we can find for each $k$ a radius $\left.\sigma_{k} \in\right] t, \frac{3}{2}\left[\right.$ on which $\mathbf{M}^{p}\left(\left\langle\mathbf{T}_{\tilde{F}_{k}}-\bar{T}_{k}, \varphi_{k}, \sigma_{k}\right\rangle\right) \leq$ $C \mathbf{h}_{k}^{2+\gamma}$. Recall from Lemma 6.1(i), $\partial\left\langle\mathbf{T}_{\tilde{F}_{k}}-\bar{T}_{k}, \varphi_{k}, \sigma_{k}\right\rangle=0 \bmod (p)$. By the isoperimetric inequality $\bmod (p)\left(\operatorname{see}\left[\operatorname{Fed} 69,(4.2 .10)^{\nu}\right]\right)$ there is an integer rectifiable current $S_{k}$, which can be assumed to be representative $\bmod (p)$, such that

$$
\begin{aligned}
& \partial S_{k}=\left\langle\mathbf{T}_{\tilde{F}_{k}}-\bar{T}_{k}, \varphi_{k}, \sigma_{k}\right\rangle \bmod (p), \quad \mathbf{M}\left(S_{k}\right)=\mathbf{M}^{p}\left(S_{k}\right) \leq C \mathbf{h}_{k}^{(2+\gamma) m /(m-1)} \\
& \quad \text { and } \operatorname{spt}\left(S_{k}\right) \subset \bar{\Sigma}_{k} .
\end{aligned}
$$

Our competitor current is, then, given by

$$
Z_{k}:=\bar{T}_{k}\left\llcorner\left(\mathbf{p}_{k}^{-1}\left(\overline{\mathcal{M}}_{k} \backslash \mathcal{B}_{\sigma_{k}}\right)\right)+S_{k}+\mathbf{T}_{\tilde{F}_{k}}\left\llcorner\left(\mathbf{p}_{k}^{-1}\left(\mathcal{B}_{\sigma_{k}}\right)\right) .\right.\right.
$$

The computations given in [DLS16b, Section 7.4] would then imply that the $p$-mass of $Z_{k}$ is strictly smaller than the mass of $\bar{T}_{k}$ for $k$ large enough, even though $\bar{T}_{k}-Z_{k}$ is a cycle $\bmod (p)$ supported in the ambient manifold $\bar{\Sigma}_{k}$, which is a contradiction to $\bar{T}_{k}$ being a mass minimizing current $\bmod (p)$ in $\bar{\Sigma}_{k}$. 
28.2 Proof of Theorem 28.3. We argue by contradiction and assume that:

$$
\begin{array}{ll}
\mathcal{H}_{\infty}^{m-2+\alpha}(\Upsilon)<\frac{\eta}{2} & \text { if } Q<\frac{p}{2}, \\
\mathcal{H}_{\infty}^{m-1+\alpha}(\Upsilon)<\frac{\eta}{2} & \text { if } Q=\frac{p}{2} .
\end{array}
$$

Since $\Upsilon$ is compact, we cover $\Upsilon$ with finitely many balls $\left\{\mathbf{B}_{\sigma_{i}}\left(x_{i}\right)\right\}$ in such a way that

$$
\begin{array}{ll}
\sum_{i} \omega_{m-2+\alpha}\left(4 \sigma_{i}\right)^{m-2+\alpha} \leq \frac{\eta}{2} & \text { if } Q<\frac{p}{2}, \\
\sum_{i} \omega_{m-1+\alpha}\left(4 \sigma_{i}\right)^{m-1+\alpha} \leq \frac{\eta}{2} & \text { if } Q=\frac{p}{2} .
\end{array}
$$

Choose a $\bar{\sigma}>0$ so that the $5 \bar{\sigma}$-neighborhood of $\Upsilon$ is covered by $\left\{\mathbf{B}_{\sigma_{i}}\left(x_{i}\right)\right\}$. Denote by $\Lambda_{k}$ the set of multiplicity $Q$ points of $\bar{T}_{k}$ far away from the singular set $\Upsilon$ :

$$
\Lambda_{k}:=\left\{q \in \mathrm{D}_{Q}\left(\bar{T}_{k}\right) \cap \mathbf{B}_{1}: \operatorname{dist}(q, \Upsilon)>4 \bar{\sigma}\right\}
$$

Clearly,

$$
\begin{aligned}
\mathcal{H}_{\infty}^{m-2+\alpha}\left(\Lambda_{k}\right) \geq \frac{\eta}{2} & \text { when } Q<\frac{p}{2}, \\
\mathcal{H}_{\infty}^{m-1+\alpha}\left(\Lambda_{k}\right) \geq \frac{\eta}{2} & \text { when } Q=\frac{p}{2} .
\end{aligned}
$$

As in [DLS16b, Section 6.2] we denote by $\mathbf{V}$ the neighborhood of $\Upsilon$ of size $2 \bar{\sigma}$. Agruing as in [DLS16b, Section 6.2, Step 1] we conclude the existence of a positive constant $\vartheta$ such that, for every fixed parameter $\sigma<\bar{\sigma}$, there is a $k_{0}(\sigma)$ such that the following estimate holds for every $k \geq k_{0}(\sigma)$. In the case $Q<\frac{p}{2}$ we have

$$
f_{\mathcal{B}_{2 \sigma}(x)} \mathcal{G}\left(\bar{N}_{k}, Q \llbracket \boldsymbol{\eta} \circ \bar{N}_{k} \rrbracket\right)^{2} \geq \vartheta \mathbf{h}_{k}^{2} \quad \forall x \in \Xi_{k}:=\mathbf{p}_{\overline{\mathcal{M}}_{k}}\left(\Lambda_{k}\right),
$$

whereas in the case $Q=\frac{p}{2}$ we have

$$
f_{\mathcal{B}_{2 \sigma}(x)} \mathcal{G}_{s}\left(\bar{N}_{k}, Q \llbracket \boldsymbol{\eta} \circ \bar{N}_{k} \rrbracket\right)^{2} \geq \vartheta \mathbf{h}_{k}^{2} \quad \forall x \in \Xi_{k}:=\mathbf{p}_{\overline{\mathcal{M}}_{k}}\left(\Lambda_{k}\right) .
$$

Indeed the argument in [DLS16b, Section 6.2] uses only the Hölder continuity of the Dir-minimizing map $N_{\infty}^{b}$ (which is a consequence of [DLS11, Theorem 2.9] for $Q<\frac{p}{2}$ and a consequence of [DLHMS, Theorem 8.1] when $Q=\frac{p}{2}$ ) and the strong convergence proved in Theorem 28.2 .

Next, following [DLS16b, Section 6.2, Step 2], for every $q \in \Lambda_{k}$ we define $\bar{z}_{k}(q)=$ $\mathbf{p}_{\pi_{k}}(q)$ (where $\pi_{k}$ is the reference plane for the center manifold related to $T_{j(k)}$ ) and

$$
\bar{x}_{k}(q):=\left(\bar{z}_{k}(q), \bar{r}_{k}^{-1} \varphi_{j(k)}\left(\bar{r}_{k} \bar{z}_{k}(q)\right)\right) .
$$


Observe that $\bar{x}_{k}(q) \in \overline{\mathcal{M}}_{k}$. We next claim the existence of a suitably chosen geometric constant $1>c_{0}>0$ (in particular, independent of $\sigma$ ) such that, when $k$ is large enough, for each $q \in \Lambda_{k}$ there is a radius $\varrho_{q} \leq 2 \sigma$ with the following properties:

$$
\begin{aligned}
& \frac{c_{0} \vartheta}{\sigma^{\alpha}} \mathbf{h}_{k}^{2} \leq \frac{1}{\varrho_{q}^{m-2+\alpha}} \int_{\mathcal{B}_{\varrho_{q}}\left(\bar{x}_{k}(q)\right)}\left|D \bar{N}_{k}\right|^{2}, \\
& \mathcal{B}_{\varrho_{q}}\left(\bar{x}_{k}(q)\right) \subset \mathbf{B}_{4 \varrho_{q}}(q) .
\end{aligned}
$$

The argument given in [DLS16b, Section 6.2, Step 2] can be routinously modified in our case. In particular we define the points $q_{k}:=\bar{r}_{k} q, z_{k}:=\bar{r}_{k} \bar{z}_{k}(q)$ and $x_{k}=$ $\bar{r}_{k} \bar{x}_{k}(q)=\left(z_{k}, \varphi_{j(k)}\left(z_{k}\right)\right)$ and discuss the three different possibilities depending on whether $z_{k}$ belongs to a cube $L \in \mathscr{W}^{j(k)}$ or to the contact set $\Gamma_{j(k)}$.

The first case, $z_{k} \in L \in \mathscr{W}_{h}^{j(k)}$ can be excluded with the same argument given in [DLS16b, Section 6.2, Step 2], where we replace [DLS16a, Proposition 3.1] with Proposition 17.26, because $q_{k}$ is a multiplicity $Q$ point for the current $T_{j(k)}$.

Following the argument in [DLS16b, Section 6.2, Step 2], when $z_{k} \in \mathscr{W}_{n}^{j(k)} \cup \mathscr{W}_{e}^{j(k)}$ we find a $t(q) \leq \sigma$ with the property that

$$
f_{\mathcal{B}_{\bar{s} t(q)}\left(\bar{x}_{k}(q)\right)} \mathcal{G}_{\square}\left(\bar{N}_{k}, Q \llbracket \boldsymbol{\eta} \circ \bar{N}_{k} \rrbracket\right)^{2} \leq \frac{\vartheta}{4 \omega_{m} t(q)^{m-2}} \int_{\mathcal{B}_{t(q)}\left(\bar{x}_{k}(q)\right)}\left|D \bar{N}_{k}\right|^{2}
$$

(where $\square=s$ for $Q=\frac{p}{2}$ and $\square=$ for $Q<\frac{p}{2}$ ) and

$$
\left|q-\bar{x}_{k}(q)\right|<\bar{s} t(q)
$$

In the argument [DLS16b, Section 6.2] we take care of substituing [DLS16a, Proposition 3.5], [DLS16b, Lemma 6.1] and [DLS16b, Proposition 3.6] respectively with Proposition 17.30, Lemma 28.1 and Proposition 17.31.

In the case $z_{k} \in \Gamma_{j(k)}$ we find a $t(q)<\sigma$ such that

$$
\int_{\mathcal{B}_{\bar{s} t(q)}\left(\bar{x}_{k}(q)\right)} \mathcal{G}_{\square}\left(\bar{N}_{k}, Q \llbracket \boldsymbol{\eta} \circ \bar{N}_{k} \rrbracket\right)^{2} \leq \frac{\vartheta}{4} \mathbf{h}_{k}^{2},
$$

whereas we observe that (28.25) holds trivially because the left hand side vanishes.

By $(28.25)$, for any $\left.\left.\varrho_{q} \in\right] s \bar{t}(q), 2 \sigma\right]$ the inclusion (28.23) holds. The argument is then closed by showing that (28.22) must hold for at least one $\left.\left.\varrho_{q} \in\right] \bar{s} t(q), 2 \sigma\right]$. The rest of the argument in [DLS16b, Section 6.2, Step 2] uses the Poincaré inequality in the $\mathcal{A}_{Q}$-valued setting to show that, under the assumption that (28.22) fails for every $\varrho \in] \bar{s} t(q), 2 \sigma],(28.26)$ and (28.24) would be incompatible with (28.20). This argument then settles the proof of the existence of $\varrho_{q}$ satsifying (28.22)-(28.23) when $Q<\frac{p}{2}$. Since the analogous Poincaré inequality can be easily seen to hold in the $\mathscr{A}_{Q}$-valued case, we easily conclude that the same argument applies when $Q=\frac{p}{2}$ exploiting the case $\square=s$ for (28.24) and (28.26) against (28.21). 
From (28.22) to (28.23) we can use the covering argument of [DLS16b, Step 3] to conclude that the inequality (28.18) and (28.19) would force a large Dirichlet energy of $\bar{N}_{k}$ on $\mathcal{B}_{3 / 2}$, in particular

$$
\begin{array}{ll}
\frac{\eta}{2} \leq \frac{C_{0}}{c_{0}} \frac{\sigma^{\alpha}}{\vartheta \mathbf{h}_{k}^{2}} \int_{\mathcal{B}_{\frac{3}{2}}}\left|D \bar{N}_{k}\right|^{2} & \text { for } Q<\frac{p}{2}, \\
\frac{\eta}{2} \leq \frac{C_{0}}{c_{0}} \frac{\sigma^{1+\alpha}}{\vartheta \mathbf{h}_{k}^{2}} \int_{\mathcal{B}_{\frac{3}{2}}}\left|D \bar{N}_{k}\right|^{2} & \text { for } Q=\frac{p}{2},
\end{array}
$$

where $C_{0}, c_{0}$ and $\vartheta$ are fixed (namely independent of $\sigma$ ). Therefore, $\sigma$ can be chosen very small, with the inequality being satisfied only for $k \geq k(\sigma)$. However, the arbitrariness of $\sigma$ and (28.7) would be incompatible with $\eta>0$, thus leading to the required contradiction.

\section{Part 5. Rectifiability of the singular set and structure theorem}

\section{Rectifiability of the singular set: proof of Theorem 1.9}

We start by introducing the term "area minimizing cones $\bmod (p)$ " for area minimizing currents $\bmod (p)$ without boundary $\bmod (p)$ which have a representative $T_{0}$ which is a cone in the sense of Corollary 7.3(iii). Such cone will be called flat if it is supported in some $m$-dimensional plane $\pi \subset \mathbb{R}^{m+n}$. We recall that, by Corollary 7.3, any flat area minimizing cone $\bmod (p)$ is congruent $\bmod (p)$ to $Q \llbracket \pi \rrbracket$, where $\pi$ is an $m$-dimensional plane and $Q$ is an integer with $0 \leq Q \leq \frac{p}{2}$. For odd $p$ we then conclude that $|Q| \leq \frac{p-1}{2}$.

Recall the definition of $k$-symmetric cones given in Definition 8.3. Following [NV], we introduce next the following terminology, which introduces a suitable notion of local almost symmetry for a given integral varifold $V$.

Definition 29.1 An m-dimensional integral varifold $V$ is $(k, \varepsilon)$-symmetric in the ball $\mathbf{B}_{\rho}(x)$ if there is a $k$-symmetric cone $C$ such that the varifold distance between $C\left\llcorner\mathbf{B}_{1}(0)\right.$ and $\left(\left(\iota_{x, \rho}\right)_{\sharp} V\right)\left\llcorner\mathbf{B}_{1}(0)\right.$ is smaller than $\varepsilon$.

Next, given a varifold $V$ with bounded mean curvature in an open set $U$, for every $\sigma>0$ and $\varepsilon>0$ we introduce the set

$\mathcal{S}_{\varepsilon}^{k, \sigma}(V):=\left\{x \in \operatorname{spt}(V) \cap U: V\right.$ is not $(k+1, \varepsilon)$-symmetric in $\mathbf{B}_{r}(x)$ for $\left.\left.\left.r \in\right] 0, \sigma\right]\right\}$

The following is then a direct corollary of Lemma 8.6.

Corollary 29.2 Assume that $T$ is as in Theorem 1.4, and consider the varifold $\mathbf{v}(T)$ induced by $T$. If $p$ is odd, then for every compact $K$ with $K \cap \operatorname{spt}^{p}(\partial T)=\emptyset$ there are constants $\varepsilon=\varepsilon(m, n, p, K)>0$ and $\sigma=\sigma(m, n, p, K)>0$ such that

$$
\operatorname{Sing}(T) \cap K \subset \bigcup_{Q=2}^{\frac{p-1}{2}} \operatorname{Sing}_{Q}(T) \cup \mathcal{S}_{\varepsilon}^{m-1, \sigma}(\mathbf{v}(T)) \cup \mathcal{S}^{m-2}(\mathbf{v}(T)) .
$$


Proof Consider a point

$$
q \in(\operatorname{Sing}(T) \cap K) \backslash\left(\bigcup_{Q=2}^{\frac{p-1}{2}} \operatorname{Sing}_{Q}(T) \cup \mathcal{S}^{m-2}(\mathbf{v}(T))\right) .
$$

We then know that at least one tangent cone in $q$ is $(m-1)$-symmetric but not flat. Therefore we know from Lemma 8.6 that $\Theta(T, q) \geq \frac{p}{2}$. We also know that $\mathbf{v}(T)$ is a varifold with bounded mean curvature (the $L^{\infty}$ bound depending only on the second fundamental form of $\Sigma$ ) and that there is a $\sigma_{0}(K)>0$ such that $\operatorname{dist}\left(q, \operatorname{spt}^{p}(\partial T)\right) \geq$ $\sigma_{0}$. In particular, by the monotonicity formula, there is a $\sigma(K, \Sigma)>0$ such that

$$
\left.\left.\|\mathbf{v}(T)\|\left(\mathbf{B}_{r}(q)\right) \geq\left(\frac{p}{2}-\frac{1}{4}\right) \omega_{m} r^{m} \quad \forall r \in\right] 0, \sigma\right] .
$$

On the other hand, if $\mathbf{v}(T)$ were $(m, \varepsilon)$-symmetric in $\mathbf{B}_{r}(q)$, then there would be a positive integer $Q$ and an oriented $m$-dimensional plane $\llbracket \pi \rrbracket$ such that the varifold distance between $\left(\left(\iota_{q, r}\right)_{\sharp} \mathbf{v}(T)\right)\left\llcorner\mathbf{B}_{1}(0)\right.$ and $Q \mathbf{v}(\llbracket \pi \rrbracket)\left\llcorner\mathbf{B}_{1}(0)\right.$ is smaller than $\varepsilon$. By the compactness Proposition 5.2 (observing that $r^{-m} \mathbf{M}\left(T\left\llcorner\mathbf{B}_{r}(x)\right)\right.$ can be bounded uniformly for $x \in K)$, when $\varepsilon$ is sufficiently small, $Q \llbracket \pi \rrbracket$ must be a representative of an area minimizing current $\bmod (p)$ and as such we must have $Q \leq \frac{p-1}{2}$. In particular, if $\varepsilon$ is sufficiently small, we would conclude

$$
\|\mathbf{v}(T)\|\left(\mathbf{B}_{r}(q)\right) \leq\left(\frac{p}{2}-\frac{3}{8}\right) \omega_{m} r^{m} .
$$

This is however not possible because of (29.1) and hence we deduce that $q \in$ $\mathcal{S}_{\varepsilon}^{m-1, \sigma}(\mathbf{v}(T))$.

Proof of Theorem 1.9 Observe that, by Almgren's stratification theorem, $\mathcal{S}^{m-2}(\mathbf{v}(T))$ has Hausdorff dimension at most $m-2$. Similarly,

$$
\bigcup_{Q=2}^{\frac{p-1}{2}} \operatorname{Sing}_{Q}(T)
$$

has Hausdorff dimension at most $m-2$ by Theorem 1.7. Since by [NV, Theorem 1.4], $\mathcal{S}_{\varepsilon}^{m-1, \sigma}(\mathbf{v}(T)) \cap K$ has finite $\mathcal{H}^{m-1}$ measure and it is $(m-1)$-rectifiable, the claim follows from Corollary 29.2.

\section{Structure theorem: proof of Corollary 1.10}

In this section we prove Corollary 1.10. First of all observe that each connected component $\Lambda_{i}$ is necessarily a regular submanifold because, by definition, it is contained in the set of regular interior points of $T$. Clearly $\Lambda_{i}$ is locally orientable, and it is simple to show that, since $p$ is odd, there is in fact a smooth global orientation. 
Clearly $T\left\llcorner\Lambda_{i}=Q_{i} \llbracket \Lambda_{i} \rrbracket \bmod (p)\right.$ for some integer multiplicity $Q_{i} \in\left[-\frac{p}{2}, \frac{p}{2}\right]$ by the constancy lemma $\bmod (p)$. On the other hand we can reverse the orientation to assume that $Q_{i} \in\left[1, \frac{p}{2}\right]$. Point (b) is then obvious because $T\left\llcorner U=\sum_{i} T_{i}\llcorner U \bmod (p)\right.$ and in fact

$$
\|T\|\left\llcorner U=\sum_{i}\left\|T_{i}\right\|\llcorner U\right.
$$

Now consider $U$ as in part (a) of the statement and observe that, by the monotonicity formula, there are constants $M(U)$ and $\rho(U)>0$, such that

$$
\left.\left.\|T\|\left(\mathbf{B}_{r}(x)\right) \leq M r^{m} \quad \forall x \in U \text { and } \forall r \in\right] 0, \rho(U)\right] .
$$

Fix a $T_{i}$ and note that, by (30.1),

$$
\left\|T_{i}\right\|\left(\mathbf{B}_{r}(x)\right) \leq M r^{m}
$$

Observe that

$$
\operatorname{spt}\left(\left(\partial T_{i}\right)\llcorner U) \subset \operatorname{Sing}(T) \cap \bar{U}=: K,\right.
$$

and that, by Theorem 1.9, the compact set $K$ satisfies the bound

$$
\mathcal{H}^{m-1}(K)<\infty \text {. }
$$

We next claim that, by (30.2) and (30.3),

$$
\mathbf{M}\left(\left(\partial T_{i}\right)\llcorner U)<\infty .\right.
$$

First of all fix $\sigma=\frac{1}{k}<\frac{\rho(U)}{2}$ and choose a finite cover of $K$ with balls $\left\{B_{j}^{k}\right\}_{j}$ with radii $r_{j}^{k}$ satisfying $2 r_{j}^{k} \leq \sigma=\frac{1}{k}$ such that

$$
\sum_{j} \omega_{m-1}\left(r_{j}^{k}\right)^{m-1} \leq 2 \mathcal{H}_{\sigma}^{m-1}(K) \leq 2 \mathcal{H}^{m-1}(K) .
$$

For each ball $B_{j}^{k}$ we choose a smooth cutoff function $\varphi_{j}^{k}$ which vanishes identically on $B_{j}^{k}$ and it is identically equal to 1 on the complement of the concentric ball $2 B_{j}^{k}$ with twice the radius. We choose $\varphi_{j}^{k}$ so that $0 \leq \varphi_{j}^{k} \leq 1$ and $\left\|d \varphi_{j}^{k}\right\|_{0} \leq C\left(r_{j}^{k}\right)^{-1}$, where $C$ is a geometric constant. We then define

$$
\varphi^{k}:=\prod_{j} \varphi_{j}^{k}
$$

Recall that

$$
\mathbf{M}\left(\left(\partial T_{i}\right)\llcorner U)=\sup \left\{\partial T_{i}(\omega):\|\omega\|_{c} \leq 1, \omega \in \mathcal{D}^{k}(U)\right\} .\right.
$$

We therefore fix a smooth $(m-1)$-form $\omega$ with compact support in $U$ and we are interested in bounding $\partial T_{i}(\omega)=T_{i}(d \omega)$. Observe that $\varphi^{k} \uparrow 1\left\|T_{i}\right\|$-a.e. on $U$. Hence we can write

$$
T_{i}(d \omega)=\lim _{k \rightarrow \infty} T_{i}\left(\varphi^{k} d \omega\right)
$$


On the other hand, since $\varphi^{k} \omega$ is supported in an open set $V \subset \subset U \backslash K$ we conclude

$$
T_{i}\left(d\left(\varphi^{k} \omega\right)\right)=\partial T_{i}\left(\varphi^{k} \omega\right)=0 .
$$

Hence we can estimate

$$
\begin{aligned}
\left|T_{i}\left(\varphi^{k} d \omega\right)\right| & =\left|T_{i}\left(d \varphi^{k} \wedge \omega\right)\right| \leq \sum_{j}\left|T_{i}\left(\prod_{\ell \neq j} \varphi_{\ell}^{k} d \varphi_{j}^{k} \wedge \omega\right)\right| \\
& \leq C \sum_{j}\|\omega\|_{c}\left\|d \varphi_{j}^{k}\right\|_{0}\left\|T_{i}\right\|\left(2 B_{j}^{k}\right) \stackrel{(30.2)}{\leq} C M\|\omega\|_{c} \sum_{j}\left(r_{j}^{k}\right)^{-1}\left(2 r_{j}^{k}\right)^{m} \\
& \leq C M\|\omega\|_{c} \mathcal{H}^{m-1}(K)
\end{aligned}
$$

Letting $k \uparrow \infty$ we thus conclude

$$
\left|T_{i}(d \omega)\right| \leq C M\|\omega\|_{c} \mathcal{H}^{m-1}(K) .
$$

This shows that $\left(\partial T_{i}\right)\llcorner U$ has finite mass. Point (a) follows therefore from the Federer-Fleming boundary rectifiability theorem.

In order to show (c), consider the set $K^{\prime}$ of points $q \in K$ where

- $K$ has an approximate tangent plane $T_{q} K$;

- $q$ is a Lebesgue point for all $\Theta_{i}$ 's with $\Theta_{i}(q) \in \mathbb{Z}$.

By a standard blow-up argument, it follows that, for every fixed $q \in K^{\prime}$, any limit $S$ of the currents $\left(\iota_{q, r}\right)_{\sharp}\left(T_{i}\right)$ as $r \downarrow 0$ is an area-minimizing current on $\mathbb{R}^{m+n}$ with boundary either $-\Theta_{i}(q) \llbracket T_{q} K \rrbracket$ or $+\Theta_{i}(q) \llbracket T_{q} K \rrbracket$. By the boundary monotonicity formula,

$$
\|S\|\left(\mathbf{B}_{1}(0)\right) \geq \frac{\left|\Theta_{i}(q)\right|}{2} \omega_{m}
$$

We therefore conclude that

$$
\liminf _{r \downarrow 0} \frac{\left\|T_{i}\right\|\left(\mathbf{B}_{r}(q)\right)}{r^{m}} \geq \omega_{m} \frac{\left|\Theta_{i}(q)\right|}{2} .
$$

Fix any natural number $N$. We then conclude from (30.2) that

$$
M \geq \lim _{r \downarrow 0} \frac{\|T\|\left(\mathbf{B}_{r}(q)\right)}{r^{m}} \geq \sum_{i=1}^{N} \liminf _{r \downarrow 0} \frac{\left\|T_{i}\right\|\left(\mathbf{B}_{r}(q)\right)}{r^{m}} \geq \sum_{i=1}^{N} \omega_{m} \frac{\left|\Theta_{i}(q)\right|}{2} .
$$

In particular we conclude that

$$
\sum_{i=1}^{\infty}\left|\Theta_{i}(q)\right| \leq \frac{2 M}{\omega_{m}} \quad \forall q \in K^{\prime} .
$$

This shows that

$$
\sum_{i} \mathbf{M}\left(\left(\partial T_{i}\right)\llcorner U) \leq \frac{2 M}{\omega_{m}} \mathcal{H}^{m-1}(K)<\infty .\right.
$$

This completes the proof of (c) and of the structure theorem. 


\section{Acknowledgments}

C.D.L. acknowledges the support of the NSF grants DMS-1946175 and DMS-1854147. A.M. was partially supported by INdAM GNAMPA research projects. The work of S.S. was supported by the NSF grants DMS-1565354, DMS-RTG-1840314 and DMS-FRG-1854344. J.H. was supported by the German Science Foundation DFG in context of the Priority Program SPP 2026 "Geometry at Infinity".

Funding Open access funding provided by Università degli Studi di Trento within the CRUI-CARE Agreement.

Open Access This article is licensed under a Creative Commons Attribution 4.0 International License, which permits use, sharing, adaptation, distribution and reproduction in any medium or format, as long as you give appropriate credit to the original author(s) and the source, provide a link to the Creative Commons licence, and indicate if changes were made. The images or other third party material in this article are included in the article's Creative Commons licence, unless indicated otherwise in a credit line to the material. If material is not included in the article's Creative Commons licence and your intended use is not permitted by statutory regulation or exceeds the permitted use, you will need to obtain permission directly from the copyright holder. To view a copy of this licence, visit http://creativecommons.org/licenses/by/4.0/.

Publisher's Note Springer Nature remains neutral with regard to jurisdictional claims in published maps and institutional affiliations.

\section{Appendix A. Proof of Proposition 11.1}

In order to reach a proof of Proposition 11.1, we will need some preliminary results. First, for a given $S \in \mathscr{R}_{1}\left(\mathbb{R}^{n}\right)$, we say that $S$ has the property $(N C)$ (no cycles) if there exists no $0 \neq R \in \mathscr{R}_{1}\left(\mathbb{R}^{n}\right)$ such that $\partial R=0$ and

$$
\mathbf{M}(S)=\mathbf{M}(R)+\mathbf{M}(S-R) .
$$

We recall that $\mathscr{I}_{m}\left(\mathbb{R}^{m+n}\right)$ denotes the space of $m$-dimensional integral currents in $\mathbb{R}^{m+n}$.

Given $S \in \mathscr{I}_{1}\left(\mathbb{R}^{n}\right)$ satisfying the property $(N C)$, we call a good decomposition of $S$ a writing

$$
S=\sum_{j=1}^{N} \theta_{j} S_{j}
$$

where $\theta_{j} \in \mathbb{N}$, each $S_{j}$ is the integral current given by $S_{j}=\llbracket \gamma_{j} \rrbracket$ for $\gamma_{j}$ a simple Lipschitz curve of finite length, $S_{j} \neq S_{k}$ if $j \neq k$ and moreover

$$
\mathbf{M}(S)=\sum_{j} \theta_{j} \mathbf{M}\left(S_{j}\right), \quad \mathbf{M}(\partial S)=\sum_{j} \theta_{j} \mathbf{M}\left(\partial S_{j}\right) .
$$


The existence of a good decomposition for a current $S \in \mathscr{I}_{1}\left(\mathbb{R}^{n}\right)$ satisfying the property $(N C)$ is a direct consequence of [Fed69, 4.2.25]. We say that a good decomposition $S=\sum_{j=1}^{N} \theta_{j} S_{j}$ has the property (NTC) (no topological cycles) if there exists no function $f:\{1, \ldots, N\} \rightarrow\{-1,0,1\}, f \not \equiv 0$, such that

$$
\partial\left(\sum_{j=1}^{N} f(j) S_{j}\right)=0 .
$$

Lemma A.1 For any $S \in \mathscr{I}_{1}\left(\mathbb{R}^{n}\right)$ with the property $(N C)$ there exists $S^{\prime} \in \mathscr{I}_{1}\left(\mathbb{R}^{n}\right)$ with the property $(N C)$ and a good decomposition of $S^{\prime}$ that satisfies $\partial S^{\prime}=\partial S$, $\mathbf{M}\left(S^{\prime}\right) \leq \mathbf{M}(S)$, and that has the property $(N T C)$.

Proof Let $S \in \mathscr{I}_{1}\left(\mathbb{R}^{n}\right)$, and assume without loss of generality that $S \neq 0$. Among all currents $S^{\prime} \in \mathscr{I}_{1}\left(\mathbb{R}^{n}\right)$ with the property $(N C)$ and such that $\partial S^{\prime}=\partial S$ and $\mathbf{M}\left(S^{\prime}\right) \leq \mathbf{M}(S)$, and among all possible good decompositions of $S^{\prime}$ not satisfying the property $(N T C)$ fix a current $S^{\prime}$ and a decomposition

$$
S^{\prime}=\sum_{j=1}^{N} \theta_{j}^{\prime} S_{j}^{\prime}
$$

such that the quantity $N$ is minimal. Observe that necessarily $N \geq 1$.

Let $f:\{1, \ldots, N\} \rightarrow\{-1,0,1\}$ be a function such that (A.2) holds. Define:

$$
j_{-} \in \operatorname{argmin}\left\{\theta_{j}^{\prime}: f(j)=-1\right\}
$$

and

$$
j_{+} \in \operatorname{argmin}\left\{\theta_{j}^{\prime}: f(j)=+1\right\} .
$$

Observe that since $S^{\prime}$ has the property $(N C)$, the sets $\left\{\theta_{j}^{\prime}: f(j)=-1\right\}$ and $\left\{\theta_{j}^{\prime}: f(j)=+1\right\}$ are non-empty.

Now, consider the quantities

$$
M_{-}:=\sum_{j: f(j)=-1} \mathbf{M}\left(S_{j}^{\prime}\right)
$$

and

$$
M_{+}:=\sum_{j: f(j)=+1} \mathbf{M}\left(S_{j}^{\prime}\right) .
$$

Clearly, if $M_{+} \geq M_{-}$then the current

$$
S_{+}^{\prime}:=S^{\prime}-\theta_{j_{+}} \sum_{j} f(j) S_{j}^{\prime}
$$


satisfies $\mathbf{M}\left(S_{+}^{\prime}\right) \leq \mathbf{M}\left(S^{\prime}\right) \leq \mathbf{M}(S)$. If instead $M_{+} \leq M_{-}$then the current

$$
S_{-}^{\prime}:=S^{\prime}+\theta_{j_{-}} \sum_{j} f(j) S_{j}^{\prime}
$$

satisfies $\mathbf{M}\left(S_{-}^{\prime}\right) \leq \mathbf{M}\left(S^{\prime}\right) \leq \mathbf{M}(S)$. In any of the two cases, $\partial S_{ \pm}^{\prime}=\partial S^{\prime}=\partial S$, and the obvious resulting decomposition of $S_{ \pm}^{\prime}$ has at most $N-1$ indexes. Hence, by minimality, the one of the two which does not increase the mass necessarily has the property $(N T C)$. This concludes the proof.

Lemma A.2 Let $S \in \mathscr{I}_{1}\left(\mathbb{R}^{n}\right)$ and $0 \neq Z \in \mathscr{R}_{0}\left(\mathbb{R}^{n}\right)$ be such that:

(H1) $A-B=\partial S+p Z$;

(H2) $S$ has the property $(N C)$ and there exists a good decomposition

$$
S=\sum_{j=1}^{N} \theta_{j} S_{j}
$$

with the property $(N T C)$.

Then, there exists $j_{0} \in\{1, \ldots, N\}$ such that $\partial S_{j_{0}}=\llbracket x \rrbracket-\llbracket y \rrbracket$ with $x, y \in \operatorname{spt}(Z)$ and $\theta_{j_{0}} \geq \frac{p}{2}$.

Proof Let $S$ and $Z$ be as above. Firstly, we claim that the set of indexes $j \in$ $\{1, \ldots, N\}$ such that $\partial S_{j}=\llbracket x \rrbracket-\llbracket y \rrbracket$ with $x, y \in \operatorname{spt}(Z)$ is non-empty. We write

$$
Z=\sum_{\ell=1}^{M} \llbracket N_{\ell} \rrbracket-\sum_{\ell=1}^{M} \llbracket P_{\ell} \rrbracket,
$$

where the $N_{\ell}$ 's (resp. the $P_{\ell}$ 's) are not necessarily distinct, so that

$$
\partial S=\sum_{i=1}^{Q} \llbracket A_{i} \rrbracket+p \sum_{\ell=1}^{M} \llbracket P_{\ell} \rrbracket-\left(\sum_{i=1}^{Q} \llbracket B_{i} \rrbracket+p \sum_{\ell=1}^{M} \llbracket N_{\ell} \rrbracket\right) .
$$

Consider any of the points $P_{\ell}$. By (A.1), the multiplicity of $\partial S$ in $P_{\ell}$ is at least $p$, and furthermore, since $Q \leq \frac{p}{2}$, there exist $j \in\{1, \ldots, N\}$ and $\ell^{\prime} \in\{1, \ldots, M\}$ such that $\partial S_{j}=\llbracket P_{\ell} \rrbracket-\llbracket N_{\ell^{\prime}} \rrbracket$, which proves our claim.

Next, assume by contradiction that for every $j$ such that $\partial S_{j}$ is supported on $\operatorname{spt}(Z)$ one has $\theta_{j}<\frac{p}{2}$. Fix, for instance, the point $P_{1}$. Arguing as above, after possibly reordering the indexes (both in the family $\left\{S_{j}\right\}$ and $\left\{N_{\ell}\right\}$ ), we conclude that there exist $N_{1}$ and $S_{1}$ such that $\partial S_{1}=\llbracket P_{1} \rrbracket-\llbracket N_{1} \rrbracket$. Moreover, by hypothesis, $\theta_{1}<\frac{p}{2}$. This ensures that we can find $P_{2}$ and $S_{2}$ such that $\partial S_{2}=\llbracket P_{2} \rrbracket-\llbracket N_{1} \rrbracket$, and again $\theta_{2}<\frac{p}{2}$. The procedure can be iterated as long as the new points $P_{\ell+1}\left(\right.$ resp. $\left.N_{\ell+1}\right)$ are distinct from the previous ones. Since the decomposition of $S$ has the property $(N T C)$ by hypothesis $(H 2)$, this would imply that the procedure can be iterated indefinitely, which gives the desired contradiction. 
Proof of Proposition 11.1 Let us first consider case (a), with $\sigma=1$.

It suffices to prove that

$$
\mathcal{F}(A-B) \leq \mathcal{F}^{p}(A-B),
$$

because the other inequality is obvious.

Suppose by contradiction that

$$
\mathcal{F}^{p}(A-B)<\mathcal{F}(A-B),
$$

and let $S \in \mathscr{I}_{1}\left(\mathbb{R}^{n}\right)$ and $0 \neq Z \in \mathscr{R}_{0}\left(\mathbb{R}^{n}\right)$ be such that

$$
A-B=\partial S+p Z \text { and } \mathbf{M}(S)<\mathcal{F}(A-B) .
$$

We claim that there exist currents $S^{1} \in \mathscr{I}_{1}\left(\mathbb{R}^{n}\right)$ and $Z^{1} \in \mathscr{R}_{0}\left(\mathbb{R}^{n}\right)$ such that

$$
A-B=\partial S^{1}+p Z^{1}, \quad \mathbf{M}\left(S^{1}\right)<\mathcal{F}(A-B) \quad \text { and } \quad \mathbf{M}\left(Z^{1}\right)=\mathbf{M}(Z)-2 .
$$

The conclusion trivially follows from the claim.

We proceed with the proof of (A.5). First observe that if $S$ has a cycle $R$ then the current $S^{\prime}:=S-R$ satisfies $A-B=\partial S^{\prime}+p Z$ and $\mathbf{M}\left(S^{\prime}\right)=\mathbf{M}(S)-\mathbf{M}(R)<$ $\mathcal{F}(A-B)$. Therefore, we can assume without loss of generality that $S$ has the property $(N C)$. Next, applying Lemma A.1 we can also assume that $S$ has a good decomposition

$$
S=\sum_{j=1}^{N} \theta_{j} S_{j}
$$

which satisfies the property $(N T C)$. Now, by Lemma A.2 there exists $j_{0} \in\{1, \ldots, N\}$ such that $\partial S_{j_{0}}=\llbracket x \rrbracket-\llbracket y \rrbracket$ with $x, y \in \operatorname{spt}(Z)$ and $\theta_{j_{0}} \geq \frac{p}{2}$. Let $S^{1}:=S-p S_{j_{0}}$. We have

$$
\partial S^{1}=\partial S-p \llbracket x \rrbracket+p \llbracket y \rrbracket,
$$

and thus

$$
A-B=\partial S^{1}+p Z^{1}
$$

where $Z^{1}:=Z+\llbracket x \rrbracket-\llbracket y \rrbracket$. The conclusion $\mathbf{M}\left(Z^{1}\right)=\mathbf{M}(Z)-2$ simply follows from (A.1). Finally, we get

$$
\mathbf{M}\left(S^{1}\right) \leq \sum_{j \neq j_{0}} \theta_{j} \mathbf{M}\left(S_{j}\right)+\left|\theta_{j_{0}}-p\right| \mathbf{M}\left(S_{j_{0}}\right) \leq \sum_{j=1}^{N} \theta_{j} \mathbf{M}\left(S_{j}\right) \stackrel{(\mathrm{A} .1)}{=} \mathbf{M}(S)<\mathcal{F}(A-B),
$$

where the second inequality follows from $\theta_{j_{0}} \geq \frac{p}{2}$.

Let us now consider instead case (b), when $\sigma=-1$ and $Q=\frac{p}{2}$. We know from (11.3) that

$$
\mathcal{F}^{p}(A+B) \leq \mathcal{F}(A+B)
$$


where $\mathcal{F}(A+B)$ is defined by (11.2). Assume by contradiction that $\mathcal{F}^{p}(A+B)<$ $\mathcal{F}(A+B)$. That is, there exist $S \in \mathscr{I}_{1}\left(\mathbb{R}^{n}\right)$ and $Z \in \mathscr{R}_{0}\left(\mathbb{R}^{n}\right)$ such that

$$
A+B=\partial S+p Z, \quad \text { and } \quad \mathbf{M}(S)<\mathcal{F}(A+B)
$$

Observe that it cannot be $Z=0$. Also, by Lemma A.1 there is no loss of generality in assuming that $S$ admits a good decomposition

$$
S=\sum_{j=1}^{N} \theta_{j} S_{j}
$$

having the property $(N T C)$. Now, if $\mathbf{M}(Z)=1$ then there exists $z \in \mathbb{R}^{n}$ such that $Z=\llbracket z \rrbracket$. In that case, if we set $R:=z \aleph_{(A+B)}$ then we have

$$
\partial R=A+B-p \llbracket z \rrbracket=\partial S,
$$

and

$$
\begin{aligned}
\mathcal{F}(A+B) \leq \mathbf{M}(R) & =\mathcal{F}(A-Q \llbracket z \rrbracket)+\mathcal{F}(B-Q \llbracket z \rrbracket) \\
& =\sum_{i=1}^{Q}\left(\left|A_{i}-z\right|+\left|B_{i}-z\right|\right) \leq \mathbf{M}(S),
\end{aligned}
$$

thus contradicting (A.6).

On the other hand, if $\mathbf{M}(Z) \geq 2$ (and thus in fact $\mathbf{M}(Z) \geq 3$ ) then there exists $j_{0} \in\{1, \ldots, N\}$ such that $\partial S_{j_{0}}=\llbracket x \rrbracket-\rrbracket y \rrbracket$ with $x, y \in \operatorname{spt}(Z)$ and $\theta_{j_{0}} \geq \frac{p}{2}$. Hence, setting $S^{1}:=S-p S_{j_{0}}$ we have

$$
A+B=\partial S^{1}+p Z^{1}
$$

with $Z^{1}:=Z+\llbracket x \rrbracket-\llbracket y \rrbracket, \mathbf{M}\left(Z^{1}\right)=\mathbf{M}(Z)-2$ and $\mathbf{M}\left(S^{1}\right) \leq \mathbf{M}(S)$. In order to complete the proof, it suffices to iterate this argument producing currents $S^{k}, Z^{k}$ until $\mathbf{M}\left(Z^{k}\right)=1$.

\section{References}

[GMT86] Some open problems in geometric measure theory and its applications suggested by participants of the 1984 AMS summer institute. In J. E. Brothers, editor, Geometric measure theory and the calculus of variations (Arcata, Calif., 1984), volume 44 of Proc. Sympos. Pure Math., pages 441-464. Amer. Math. Soc., Providence, RI, 1986.

[All72] William K. Allard. On the first variation of a varifold. Ann. of Math. (2), 95:417-491, 1972.

[Alm91] Frederick J. Almgren, Jr. Multi-functions mod $\nu$. In Geometric analysis and computer graphics (Berkeley, CA, 1988), volume 17 of Math. Sci. Res. Inst. Publ., pages 1-17. Springer, New York, 1991.

[Amb90] Luigi Ambrosio. Metric space valued functions of bounded variation. Ann. Scuola Norm. Sup. Pisa Cl. Sci. (4), 17(3):439-478, 1990. 
[AFP00] Luigi Ambrosio, Nicola Fusco, and Diego Pallara. Functions of bounded variation and free discontinuity problems. Oxford Mathematical Monographs. The Clarendon Press, Oxford University Press, New York, 2000.

[AK00] Luigi Ambrosio and Bernd Kirchheim. Currents in metric spaces. Acta Math., 185(1):1-80, 2000.

[DlHMS] Camillo De Lellis, Jonas Hirsch, Andrea Marchese, and Salvatore Stuvard. Area minimizing currents mod 2Q: linear regularity theory. Comm. Pure Appl. Math., to appear. arXiv:1909.03305.

[DLS14] Camillo De Lellis and Emanuele Spadaro. Regularity of area minimizing currents I: gradient $L^{p}$ estimates. Geom. Funct. Anal., 24(6):1831-1884, 2014.

[DLS15] Camillo De Lellis and Emanuele Spadaro. Multiple valued functions and integral currents. Ann. Sc. Norm. Super. Pisa Cl. Sci. (5), 14(4):1239-1269, 2015.

[DlS16a] Camillo De Lellis and Emanuele Nunzio Spadaro. Regularity of area minimizing currents II: center manifold. Ann. of Math. (2), 183(2):499-575, 2016a.

[DLS16b] Camillo De Lellis and Emanuele Spadaro. Regularity of area minimizing currents III: blow-up. Ann. of Math. (2), 183(2):577-617, $2016 \mathrm{~b}$.

[DLS11] Camillo De Lellis and Emanuele Nunzio Spadaro. $Q$-valued functions revisited. Mem. Amer. Math. Soc., 211(991):vi+79, 2011.

[DPH12] Thierry De Pauw and Robert HardT. Rectifiable and flat $G$ chains in a metric space. Amer. J. Math., 134(1):1-69, 2012.

[DPH14] Thierry De Pauw and Robert Hardt. Some basic theorems on flat $G$ chains. J. Math. Anal. Appl., 418(2):1047-1061, 2014.

[Fed69] Herbert Federer. Geometric measure theory. Die Grundlehren der mathematischen Wissenschaften, Band 153. Springer-Verlag New York Inc., New York, 1969.

[Fed70] HERBERT FEDERER. The singular sets of area minimizing rectifiable currents with codimension one and of area minimizing flat chains modulo two with arbitrary codimension. Bull. Amer. Math. Soc., 76:767-771, 1970.

[KP08] Steven G. Krantz and Harold R. Parks. Geometric integration theory. Cornerstones. Birkhäuser Boston, Inc., Boston, MA, 2008.

[MS18] Andrea Marchese and Salvatore Stuvard. On the structure of flat chains modulo $p$. Adv. Calc. Var., 11(3):309-323, 2018.

[Mat95] Pertti Mattila. Geometry of sets and measures in Euclidean spaces, volume 44 of Cambridge Studies in Advanced Mathematics. Cambridge University Press, Cambridge, 1995. Fractals and rectifiability.

[NV] Aaron Naber and Daniele Valtorta. The singular structure and regularity of stationary varifolds. J. Eur. Math. Soc. (JEMS), to appear. arXiv:1505.03428.

[SSA77] Richard Schoen, Leon Simon, and Frederick J. Jr. Almgren. Regularity and singularity estimates on hypersurfaces minimizing parametric elliptic variational integrals. I, II. Acta Math., 139(3-4):217-265, 1977.

[Sim93] L. Simon. Cylindrical tangent cones and the singular set of minimal submanifolds. Journal of Differential Geometry, 38(3):585-652, 1993.

[Sim83] LeOn Simon. Lectures on geometric measure theory, volume 3 of Proceedings of the Centre for Mathematical Analysis, Australian National University. Australian National University, Centre for Mathematical Analysis, Canberra, 1983. 
[Sim95] LEON Simon. Rectifiability of the singular sets of multiplicity 1 minimal surfaces and energy minimizing maps. In Surveys in differential geometry, Vol. II (Cambridge, MA, 1993), pages 246-305. Int. Press, Cambridge, MA, 1995.

[Spo19] LuCA Spolaor. Almgren's type regularity for semicalibrated currents. Adv. Math., 350:747-815, 2019.

[Tay73] JEAN E. TAYLOR. Regularity of the singular sets of two-dimensional areaminimizing flat chains modulo 3 in $R^{3}$. Invent. Math., 22:119-159, 1973.

[Whi79] BRIAN White. The structure of minimizing hypersurfaces mod 4. Invent. Math., 53(1):45-58, 1979.

[Whi86] BRIAN WhITE. A regularity theorem for minimizing hypersurfaces modulo $p$. In Geometric measure theory and the calculus of variations (Arcata, Calif., 1984), volume 44 of Proc. Sympos. Pure Math., pages 413-427. Amer. Math. Soc., Providence, RI, 1986.

[Whi97] BRIAN White. Stratification of minimal surfaces, mean curvature flows, and harmonic maps. J. Reine Angew. Math., 488:1-35, 1997.

[You18] Robert Young. Quantitative nonorientability of embedded cycles. Duke Math. J., 167(1):41-108, 2018.

C. De Lellis

School of Mathematics, Institute for Advanced Study, 1 Einstein Dr., Princeton, NJ 05840, USA. Institut für Mathematik, Universität Zürich, Winterthurerstrasse 190, Zurich 8057, Switzerland.

camillo.delellis@math.ias.edu

J. HIRSCH

Mathematisches Institut, Universität Leipzig, Augustusplatz 10, 04109 Leipzig, Germany. hirsch@math.uni-leipzig.de

A. MARCHESE

Dipartimento di Matematica, Università di Trento, via Sommarive 14, 38123 Povo, (TN), Italy.

andrea.marchese@unitn. it

S. STUVARD

Department of Mathematics, The University of Texas at Austin, 2515 Speedway, Stop C1200, Austin, TX 78712-1202, USA.

stuvard@math.utexas. edu

Received: September 23, 2019

Revised: August 15, 2020

Accepted: August 19, 2020 